\title{
Abstracts of the \\ 2020 AANS/CNS Joint Section on \\ Disorders of the Spine and Peripheral Nerves \\ Annual Meeting \\ Las Vegas, Nevada • March 5-8, 2020
}

(DOI: 10.3171/2021.3.DSN2020abstracts)

Disclaimer: The Journal of Neurosurgery Publishing Group (JNSPG) acknowledges that these abstracts are published as submitted and did not go through JNSPG's peer-review or editing process.

Friday, March 6, 2020

Kuntz Scholar Oral Abstract Presentations

Concurrent Session 1

\section{3:15 - 3:20 pm}

\section{The Risk of Peripheral Nerve Tumor Biopsy in Suspected Benign Etiologies}

Roberto Perez-Roman MD; Shelby Burks; lahn Cajigas; Luca Debs BS; Allan D. Levi MD, PhD

Introduction: Peripheral nerve sheath tumors (PNST) are tumors with unique clinical and imaging features that present to a variety of physicians. These lesions are often referred for biopsy which can put nerve fascicles at risk. Pre-operative biopsy may cause distortion of normal anatomical planes, making definitive resection difficult.

Methods: Surgical cases collected retrospectively using a prospectively established database of PNST treated by a single surgeon between 1997 and 2019. Patients were dichotomized depending on pre-operative biopsy. Effects of biopsy were assessed via history and physical examination both pre- and post-definitive resection.

Results: Total of 151 cases were included. Only $23.2 \%$ (35) of patients underwent pre-operative biopsy, but $42.9 \%$ of these experienced new or worsening neurologic exam immediately following biopsy. After definitive resection the rate of neurologic deficit was significantly different between the two groups with $60 \%$ of biopsy patients and $19 \%$ of those patients not biopsied experiencing decline in exam $(F=25.72 p<0.001)$. Odds ratio for any post-operative deficit for biopsy was $6.40(\mathrm{Cl}[2.8,14.55], \mathrm{p}<0.001)$. Univariate logistic regression of neurologic deficit with patient age, sex, tumor type, and biopsy status showed that only biopsy was associated with occurrence of any post-operative deficit.

Conclusion: Biopsy of benign PNST is associated with a high rate of neurologic deficit both immediately following the procedure and definitive resection. Careful selection its imperative prior to proceeding with biopsy of nerve sheath tumors exhibiting benign features given the unacceptably high rate of neurologic decline. 


\section{3:25 - 3:30 pm}

\section{Decompression and Fusion Versus Decompression Alone for Grade I Degenerative Lumbar Spondylolisthesis}

Andrew Kai-Hong Chan MD; Erica F. Bisson MD, MPH, FAANS; Mohamad Bydon MD; Kevin T. Foley MD, FACS, FAANS; Steven D. Glassman; Christopher I. Shaffrey MD, FACS; Eric A. Potts MD; Mark E. Shaffrey MD , FAANS, FACS; Domagoj Coric MD; John J. Knightly MD; Paul Park MD; Michael Y. Wang MD, FAANS; Kai-Ming G. Fu MD, PhD; Jonathan Slotkin MD; Anthony L. Asher MD, FACS; Michael S. Virk MD, PhD; Panagiotis Kerezoudis; Mohammed A. Alvi MD; Jian Guan MD; Regis W. Haid; Praveen V. Mummaneni MD

Introduction: Prior studies including two recent randomized clinical trialshave not revealed the ideal surgical management strategy for degenerative lumbar spondylolisthesis.

Methods: Twelve top-enrolling QOD sites formed a spondylolisthesis study group to investigate the effectiveness of fusion for grade 1 degenerative lumbar spondylolisthesis. From 7/2014 through 6/2016, 608 patients underwent single-segment surgery for grade 1 degenerative lumbar spondylolisthesis at these sites. Demographics, clinical characteristics, surgical variables, and radiographic parameters were collected. The primary outcome, 24-month Oswestry Disability Index (ODI) change, was analyzed with multivariable linear regression. Other secondary outcomes included the proportion reaching ODI minimal clinically important difference (MCID; 12.8 point change), Numeric Rating Scale (NRS) Back Pain (NRS-BP), NRS Leg Pain (NRS-LP), EuroQol-5D (EQ-5D), NASS Satisfaction, and reoperation rates.

Results: Of the 608 patients (85.5\% 24-month follow-up rate), 140 (23.0\%) underwent decompression alone and $468(77.0 \%)$ underwent decompression and fusion. The 24-month ODI change was significantly greater in the fusion group $(25.8 \pm 20.0$ vs. $15.2 \pm 19.8, p<0.001)$. Fusions were significantly more likely to reach ODI MCID at 24 months $(73.3 \%$ vs. $56.0 \%, p \leq 0.001)$. Reoperation rates were similar for fusion and decompression-only groups $(6.2 \%$ vs. $9.3 \%$, respectively, $p=0.206)$. In multivariable analyses, the addition of fusion was independently associated with superior ODI change $(B=7.05,95 \% \mathrm{Cl}(10.70$ to 3.39$), \mathrm{p}<0.001)$, ability to achieve ODI MCID (OR=1.77, 95\% Cl(1.06-2.94), $\mathrm{p}=0.03)$, NRS-BP change $(\mathrm{B}=1.18,95 \% \mathrm{Cl}(0.61-1.76)$, $\mathrm{p}<0.001)$, and NASS satisfaction (OR=2.22, 95\% $\mathrm{Cl}(1.41-3.45), \mathrm{p}<0.001)$ at 24 months. Type of procedure was not associated with NRS-LP or EQ-5D change ( $p>0.05)$.

Conclusion: The results of our study suggest that decompression with fusion may offer superior outcomes compared to decompression alone in patients with grade 1 degenerative lumbar spondylolisthesis at 24 months. 
3:35 - 3:40 pm

\section{Radiological and Clinical Outcomes of Three-level Cervical Disc Arthroplasty}

Ken Hsuan-kan Chang; Jau-Ching Wu MD, PhD

Introduction: One- and two-level cervical disc arthroplasty (CDA) has been compared to ACDF in several large prospective, randomized trials that have demonstrated similar clinical outcomes. However, whether these results could be applied to three-level disc herniation or spondylosis remains elusive.

Methods: A series of 50 patients who underwent three-level CDA at C3-7 were retrospectively reviewed and compared with 50 ACDF patients. Clinical outcomes were measured by the visual analogue scale (VAS), Japanese Orthopedic Association (JOA) scores, and the Neck Disability Index (NDI). Radiographic outcomes included range of motion (ROM). Fusion was evaluated by CT in the ACDF group. Complication profiles were investigated.

Results: The demographic data were very similar. During the follow-up of 24 months, the clinical outcomes improved for both the CDA and ACDF patients when compared to that of pre-operation. There were essentially little differences between the two groups in VAS, JOA scores, and NDI at pre- and post-operation. After surgery, the CDA group had an increased mean ROM of approximately $3.4^{\circ}$ at $25.2 \pm 8.84^{\circ}$ than that at preoperation $\left(21.8 \pm 7.20^{\circ}, p=0.001\right)$, whereas the ACDF group had little mobility $(22.8 \pm 5.90$ and $1.0 \pm 1.28$, preand post-operation, respectively, $p<0.001)$. The complication profiles were similar for both groups.

Conclusion: In this selectively matched retrospective study, clinical outcomes of three-level CDA and ACDF were similar during the follow-up of two years. The CDA successfully preserved mobility at the three indexed levels. However, the safety and efficacy of three-level CDA requires more long-term data to validate. 
3:45 - 3:50 pm

\section{An ERAS Pathway Following Single-level MIS TLIF Significantly Decreases LOS}

Mena G. Kerolus MD; Ketan Yerneni BA; Christopher D. Witiw MD; Ricardo B. V. Fontes MD, PhD; Harel Deutsch MD; Richard G. Fessler MD, PhD; John E. O'Toole MD, MS

Introduction: High opioid requirements in the perioperative and postoperative recovery period in patients undergoing lumbar spine fusion surgery remains a challenge.Although minimally invasive(MIS) techniques have been developed,there still remains substantial difficulties with reducing length of hospital stay(LOS) while decreasing opioid requirements.

Methods: We implemented an ERAS pathway in patients undergoing an elective one-level MIS-TLIF for degenerative changes at a single institution in January 2016.Consecutive eligible patients were enrolled over a 12-month period.This group was compared with a control cohort which was comprised of consecutive patients undergoing a one-level MIS TLIF over the 2-years prior to the implementation of the ERAS protocol.The primary outcome was LOS.Secondary outcomes included reduction in morphine equivalent units(MEU), pain scores, postoperative urinary retention, and incidence of postoperative delirium.Patients were compared using the chi-square and student t-tests.

Results: Two hundred and ninety nine patients were evaluated in this study,87 in the ERAS group and 212 in the control group. There was a significant decrease in LOS in the ERAS cohort(3.1 days vs 3.7 days, $p=$ 0.006), a significant reduction in morphine equivalent units during the course of the hospitalization in the ERAS cohort (241.3 MEU vs 360.8 MEU, $p=0.003$ ), and there was a significant reduction in the number of patients with postoperative urinary retention in the ERAS cohort (42 vs $138(p=0.01)$. There were no significant differences in incidence of delirium or pain scores.

Conclusion: This is the largest ERAS MIS fusion cohort published to date evaluating a single cohort of patients in a generalizable manner. This ERAS pathway has shown substantial decrease in LOS and opioid requirements in the immediate perioperative and postoperative period. There is further work to be done to evaluate patients other spine surgical interventions. 


\section{3:55 - 4:00 pm}

\section{Outcomes and Complications with Age in Spondylolisthesis: An Evaluation from the Quality Outcomes Database}

Michael Karsy MD, PhD, MSc; Andrew Kai-Hong Chan MD; Praveen V. Mummaneni MD; Michael S. Virk MD PhD; Mohamad Bydon MD; Steven D. Glassman; Kevin T. Foley MD, FACS, FAANS; Matthew B. Potts; Christopher I. Shaffrey MD, FACS; Mark E. Shaffrey MD, FAANS, FACS; Anthony L. Asher MD, FACS; John J. Knightly MD; Paul Park MD; Kai-Ming G. Fu MD, PhD; Jonathan Slotkin MD; Regis W. Haid; Michael Y. Wang MD FAANS; Erica F. Bisson MD, MPH, FAANS

Introduction: Degenerative lumbar spondylolisthesis affects $320 \%$ of the population and up to $30 \%$ of the elderly. There is not yet consensus on whether age is a contraindication for surgical treatment of elderly patients.

Methods: The Quality Outcomes Database multicenter, prospective registry was used to evaluate patients from 12 U.S. centers, including academic and private institutions, who underwent surgical treatment for grade 1 lumbar spondylolisthesis between July 2014 and June 2016.

Results: A total of 608 patients who fit the inclusion criteria were categorized by age $<60(n=239), 60-70$ $(n=209), 71-80(n=128)$, and $>80(n=32)$ years. Older patients showed lower mean body mass index $(p=0.00001)$ and higher rates of diabetes $(p=0.007)$, coronary artery disease $(p=0.0001)$, and osteoporosis $(p=0.005)$. A lower likelihood for home disposition was seen with higher age $(89.1 \%$ in $<60$-year-old vs. $75 \%$ in $>80$-year-old patients; $p=0.002$ ). There were no baseline differences in PROs (Oswestry Disability Index, EQ$5 \mathrm{D}$, Numeric Rating Scale for leg pain and back pain) among age categories. A significant improvement for all PROs was seen regardless of age $(p<0.05)$, and most patients met minimal clinically important differences (MCIDs) for improvement in postoperative PROs. No differences in hospital readmissions or reoperations were seen among age groups $(p<0.05)$. Multivariate analysis demonstrated that, after controlling other variables, a higher age did not decrease the odds of achieving MCID at 12 months for the PROs.

Conclusion: Our results indicate that well-selected elderly patients undergoing surgical treatment of grade 1 spondylolisthesis can achieve meaningful outcomes. This modern, multicenter U.S. study reflects the current use and limitation of spondylolisthesis treatment in the elderly, which may be informative to patients and providers. 
4:05 - 4:10 pm

\section{Effect of Cage Type on Radiographic Outcomes in TLIF}

Yaroslav J. Gelfand MD; Joshua Benton BA; Rafael De la Garza Ramos MD; Vijay Yanamadala MD, MBA; Reza Yassari MD, MS; Merritt D. Kinon MD

Introduction: The effect of expandable versus static cage type on radiographic outcomes in transforaminal lumbar interbody fusion (TLIF) have not been well studies in the literature.

Methods: We conducted a retrospective review of patients who underwent TLIF in a single institution from 2014-2019. Three cage types were identified: banana cage, bullet cage (titanium or PEEK), and expandable cage. CT scans of lumbar spine or lumbar X-rays (when CT was not available) with at least 6 weeks follow up time were used for radiographic assessment.

Results: 100 patients with 133 fused segments were identified. Average age was 60.9 , and $40 \%$ were male. Bana cage was used in 19 segments (14.3\%), bullet cage in $47(35.4 \%)$, and expandable cage in 67 segments $(50.4 \%)$. There were no significant differences in $\mathrm{FH}$ (average increase $0.7 \mathrm{~mm}, \mathrm{p}=0.771$ ), subsidence (avergae $2.3 \mathrm{~mm}, \mathrm{p}=0.554), \mathrm{DH}$ (average decrease of $1.0 \mathrm{~mm}, \mathrm{p}=0.769)$, or clinically significant subsidence $(>4 \mathrm{~mm}$, present in $26.3 \%$ on average, $p=0.174)$. SL was significantly different with banana cage $(-0.8$ degrees) and bullet cage ( -1.8 degrees) showing a decrease in segmental lordosis, but expandable cages demonstrating positive 1.2 degree gain in lordosis $(p=0.05)$. On multivariate expandable cage was significantly better for segmental lordosis $(p=0.031)$.

Conclusion: An overall average increase in the foraminal height in TLIF is small $(0.7 \mathrm{~mm})$ and is not different between the cage types, thus direct decompression is crucially important in this procedure. While TLIF is often considered to be a kyphotic procedure, an expandable cage demonstrated superiority in terms of segmental lordosis improvement and should be considered when such correction is desired. 
4:15 - 4:20 pm

\section{Could Botulinum Toxin Pre-conditioning be an Ancillary Therapy to Improve Motor Nerve Transfer Surgery?}

Nikhil Murthy MD; Dominic D'Andrea; Audrey Leung; Colin Franz

Introduction: Peripheral axon regeneration is improved when the nerve lesion under consideration has recently been preceded by another nerve injury. This is known as the conditioning lesion effect (CLE). While the CLE is one of the most robust and well characterized means to enhance motor axon regeneration in experimental models, it is not considered a clinically feasible strategy. A pharmacological means to re-produce the CLE is highly desirable in the clinical setting.

Methods: We examined the effects of a 1-week preconditioning administration of BoTX on motor axon regrowth in both a mouse tibial nerve injury and human embryonic stem cell (hESC)-based model. In-vivo neuronal reinnervation was assessed with retrograde tracers and histological analysis of peripheral nerve tissue after injections into the triceps surae muscle group. In-vitro motor neuron neurite outgrowth was assessed after incubation of HESC in BoTX by immunohistochemistry and morphometric analysis. Additionally, we used microfluidic channels to examine morphologic innervation of hESC motor neurons with human primary satellite cell-derived myotubes.

Results: BoTX conditioning treatment enhanced outgrowth of both murine motor axons in vivo $(p=0.004)$ and human MN neurites in vitro $(p<0.001)$. Microfluidic channel experiments showed a trend towards increased micro-channel "innervation" in the BoTX group $(p=0.071)$.

Conclusion: BoTX preconditioning represents a candidate for a pharmacological intervention to enhance motor axon regeneration in specific clinical scenarios, such as nerve transfer surgery. Further studies are underway to address this in a pre-clinical model of nerve transfer surgery as well as to elucidate its molecular mechanism. 
Friday, March 6, 2020

Kuntz Scholar Oral Abstract Presentations

Concurrent Session 2

\section{3:15 - 3:20 pm}

\section{Development of Predictive Model for 30-days Complications Following Posterior Cervical Fusion}

Nida Fatima; John H. Shin MD

Introduction: Perioperative prognostication of the adverse events following posterior cervical fusion (PCF) is critical to be able to implement targeted efforts in limiting these complications

Methods: A total of 3,210 patients from the American College of Surgeons, National Surgical Quality Improvement Program who underwent PCF between 2005 and 2017 were extracted. Multivariable logistic regression analysis (MLR) model was used to evaluate the association between peri-operative clinical characteristics and complications following PCF. All associations were computed as odds ratio (OR) with 95\% confidence interval $(\mathrm{Cl})$.

Results: The 30 -d complications for the 3,210 patients (48.8\% females, mean age: $63.9 \pm 13.5$ years) following PCF was 400 (12.4\%). The most common complications were bleeding disorders $(n=395,12.3 \%)$, reoperation $(n=178,5.5 \%)$, urinary tract infection $(n=83,2.6 \%)$, and pneumonia $(n=82,2.6 \%)$. Risk factors of any $30-d$ complications following PCF included bleeding disorders (OR: 2.78, 95\% Cl: 1.00-7.67, $p=0.05$ ), preoperative blood transfusions (OR: 8.76, 95\%Cl: 2.59-29.6, p<0.001), American Society of Anesthesiologists (ASA) II (OR: 4.12, 95\%Cl: 1.86-9.07, p<0.001), ASA III (OR: 2.42, 95\% Cl: 1.12-4.46, p=0.02), preoperative albumin (OR: $1.01,95 \% \mathrm{Cl}: 1.003-1.01, \mathrm{p}=0.001$ ), total operative time (OR: $1.01,95 \% \mathrm{Cl}: 1.001-1.01, \mathrm{p}<0.001$ ), and duration of anesthesia (OR: 1.00, 95\% Cl: 1.001-1.002, p=0.05).

Conclusion: This is the largest study to date of the patients undergoing PCF and provides a set of predictive variables that accounts for patient and surgical-related factors which can contribute to patient-counselling and accurate risk-adjustments 


\section{3:25 - 3:30 pm}

\section{The Natural History of Degenerative Cervical Myelopathy: An Ambispective Longitudinal Cohort Study}

Allan R. Martin BASc, MD; Sukhvinder Kalsi-Ryan BScPT, MSc, PhD; Muhammad Akbar BSc, MD; Jetan H. Badhiwala; Jefferson R. Wilson MD, PhD; Lindsay Tetreault Bsc; Aria Nouri MD, MSc; Anna Rienmuller; Eric M. Massicotte MD; Michael G. Fehlings MD, PhD, FRCS(C), FACS

Introduction: Degenerative cervical myelopathy $(\mathrm{DCM})$ is the most common pathology affecting the spinal cord, but its natural history is poorly characterized. Mild DCM is often managed non-operatively, but surgery is recommended if neurological deterioration occurs.

Methods: Patients with 1) a new diagnosis of DCM or 2) recurrent myelopathy after previous surgery were enrolled prospectively and retrospective chart reviews were also performed. Patients that did not undergo surgery or had multiple clinic visits prior to surgery were included. Standard clinical assessments by the treating surgeons were used as the clinical case definition of neurological deterioration. A battery of quantitative neurological assessments were performed at one or more visits, including mJOA, QuickDASH, GRASSP-myelopathy (motor, sensory, and dexterity), grip dynamometer, Berg Balance, gait stability ratio, and gait variability index; a deterioration of $10 \%$ in any of these measures was considered significant (e.g. a 2-point decrease in mJOA). Anatomical MRI scans were assessed for evidence of worsening compression or spinal cord signal change.

Results: 116 DCM patients were included (94 newly diagnosed, 22 recurrent myelopathy). Over a mean follow-up of 2.2 years, $57 \%(95 \% \mathrm{Cl}=47-67 \%)$ of newly diagnosed and $73 \%(95 \% \mathrm{Cl}=52-87 \%)$ of recurrent DCM patients deteriorated neurologically. The most sensitive quantitative measures to detect deterioration were grip strength (60\%), GRASSP dexterity $(60 \%)$, and gait stability ratio (50\%). mJOA and anatomical MRI had relatively low sensitivity $(33 \%, 28 \%$, respectively). A composite score of clinical measures had sensitivity $=81 \%$ and specificity $=82 \%$.

Conclusion: DCM has a poor natural history with a high rate of neurological deterioration. Longitudinal monitoring of patients should include grip strength, dexterity, and gait analysis. A lack of worsening on anatomical MRI or mJOA should not be considered evidence of clinical stability. 
3:35 - 3:40 pm

\section{The Effect of Elevated Mean Arterial Blood Pressure in Cervical Traumatic Spinal Cord Injury with Hemorrhagic Contusion}

Harry M. Mushlin MD; Timothy Chryssikos MD, PhD; Noah Lessing; Nathan L. Pratt MD; Nicholas Caffes MD; Jeffrey Oliver MD; Aaron P. Wessell MD; Bizhan Aarabi MD; Gary T. Schwartzbauer MD, PhD

Introduction: The natural history of hemorrhagic contusion (HC) in cervical spinal cord injury (CSCl) is poorly understood. We investigated $\mathrm{HC}$ expansion in $\mathrm{CSCl}$ patients with a mean arterial pressure (MAP) goal $\geq 85$ $\mathrm{mm} \mathrm{Hg}$ for 7 days following injury. Neurological follow up $\geq 6$ months was reported.

Methods: A retrospective review was performed from 2005-2016 to identify motor complete SCl patients with at least two cervical MRIs within 7 days of injury with evidence of hemorrhagic contusion. T2 MRI was used to calculate hemorrhagic surface area in the sagittal plane. Basic demographic information was recorded. Calculated MAPs were recorded between the time of the first and second MRI. AIS disability score and ASIA motor score was recorded for follow up $\geq 6$ months. Univariate and multivariate regression analysis was performed.

Results: One hundred and ninety-three patients were identified. Overall mean MAP was $88.77 \mathrm{mmHg}$. Mean change in hemorrhagic area was $23.97 \mathrm{~mm}^{2}$. There were 114 AIS A and 79 AIS B patients. Multivariate regression analysis demonstrated that elevated mean arterial pressure was a non-significant predictor of expansion at $>5,>10,>25 \mathrm{~mm}^{2}$. At follow up $\geq 6$ months, post-operative MRI hemorrhagic contusion area was significantly related to an AIS improvement $\geq 2$ ( $p=0.008$; OR 0.92$)$. Mean MAP was not significantly related to neurological improvement.

Conclusion: This is the first study to provide clinical evidence for the potential safety of elevated MAP in HC. Elevated MAP in setting of motor complete SCI does not significantly increase the risk of HC expansion in $\mathrm{CSCl}$. In addition, this study does show the importance of $\mathrm{HC}$ with post-operative $\mathrm{HC}$ area being a significant predictor of AIS improvement $\geq 2$. 


\section{Ultra-Early Surgery (<12 hrs) Correlates with Improvement in Motor Recovery after Spinal Cord Injury: A TRACK-SCI Study}

John Frederick Burke MD, PhD; Anthony M. DiGiorgio DO, MHA; Michael Beattie; Jacqueline Bresnahan; Leigh H. Thomas BA; Xuan Duong Fernandez BA; Lisa U. Pascual MD; Ferguson Adam PhD; Jonathan Pan; Debra P. Hemmerle RN, MSN; Vineeta Singh; Abel Torres; Nikolaos Kyritsis PhD; Geoffrey T. Manley MD, PhD; Jason Talbott; William Whetstone; Sanjay S. Dhall MD

Introduction: Surgical timing after spinal cord injury (SCI) remains a controversial topic. Although several studies have shown that early surgery is beneficial, it is unclear how early patients should be taken to surgery. Here, we report results from a prospective trial designed to test if ultra-early surgery (<12 hours after injury) improves outcome after SCl.

Methods: Patients presented to the emergency department with acute spinal cord injury. All patients in the current study were evaluated and determined to require surgical decompression based on their injury. Exclusion criterion consisted of (1) death while admitted to the hospital, (2) inability to participate in motorexaminations, and (3) unknown time of injury. Motor scores were taken daily while patients were admitted, and the primary endpoint for the current study was the difference in motor scores between admission and discharge.

Results: 35 patients with cervical SCI were prospectively collected. The mean time to surgery in this group was 15.4 hours. We grouped patients based on whether they went to the operating room within 12 hours of injury (20 patients), between 12-24 hours of injury (8 patients), and after 24 hours of injury (7 patients). The average motor score improvement for the ultra early group (<12 hours) was 13.8 and for the remaining groups was 2.47 (two tailed t-test $p=0.06$ ).

Conclusion: Preliminary data from our prospective trial suggests that the faster patients are surgically decompressed and stablized after $\mathrm{SCl}$, the greater the motor recovery. 
3:55 - 4:00 pm

\title{
The Association of Preoperative Narxcheck Scores and Perioperative Outcomes in Posterior Cervical Surgery Patients
}

\author{
Jacob Hoffmann; Robert Winkelman BS; Michael P. Steinmetz MD; Thomas E. Mroz MD
}

Introduction: Preoperative opioid consumption is associated with increased opioid requirements and adverse perioperative outcomes following elective surgery. Quantifying opioid use can be difficult for surgical providers preoperatively, as opioids are often prescribed by multiple providers. The NarxCheck score is developed from an algorithm that analyzes state data for controlled substances.

Methods: This study is a retrospective review of posterior cervical surgery patients whohadpreoperative NarxCheck scores. Electronic medical record database was queried to obtain perioperative pain and opioid medications prescribed at discharge. Multivariable linear regression models were developed to assess the association of preoperative NarxCheck scores with perioperative visual analog scale (VAS) pain scores, dosing of discharge opioid medication (in morphine equivalent doses), and length of stay (LOS).

Results: One hundred and two patients met inclusion for the study: 74 patients with posterior cervical fusion and 28 with laminoplasty. Preoperative NarxCheck scores ranged from 0-711 with a median value of 90 (IQR: 0 to 230). Holding other variables constant in the regression model, a 150 point increase in the preoperative NarxCheck score was significantly associated with higher pre $(+0.5,95 \% \mathrm{Cl}: 0.1$ to 0.9$)$ and post-operative pain scores $(+1.0,95 \% \mathrm{Cl}: 0.5$ to 1.4$)$, lower improvement in pain scores following surgery $(-0.5,95 \% \mathrm{Cl}:-1.0$ to 0$)$, and longer LOS ( $+10 \%, 95 \% \mathrm{Cl}: 2.0$ to $22 \%)$. Preoperative NarxCheck scores were not significantly associated with amount of opioids prescribed at discharge.

Conclusion: High preoperative NarxCheck scores were associated with increased perioperative pain scores and LOS in posterior cervical surgery. Elevated NarxCheck scores may be used to identify patients at risk for increased narcotic requirements, and prompt early utilization of multimodal pain therapies and referral to pain management. 
4:05 - 4:10 pm

\section{Long-Term Radiographic Outcomes in Patients Undergoing Four and Five Level ACDF Without Posterior Supplementation}

Samuel H. Farber MD; David Mauler; Soumya Sagar; Mark A. Pacult; Corey T. Walker MD; James Zhou MD; Jay D. Turner MD, PhD; Juan S. Uribe MD, FAANS; Steve W. Chang MD; Udaya K. Kakarla MD

Introduction: Anterior cervical discectomy and fusion (ACDF) is a common neurosurgical procedure performed to treat a wide variety of pathologies in the cervical spine. Fusion rates and long term outcomes are well studied for ACDF of three levels or less, but much less is known regarding surgery on four or more segments.

Methods: We retrospectively reviewed patients who underwent four- or five-level ACDF at a single institution over a five-year period (May 2013-May 2018). Patients who underwent a planned second stage posterior fusion or an anterior corpectomy were excluded. Only those patients with greater than one year follow up were analyzed for fusion outcomes. Fusion was evaluated by two independent neurological surgeons based on dynamic radiographs and/or computed tomography.

Results: A total of 148 patients were included ( 4 level $=142,5$ level $=6$ ). The average age was 60.4 years and $51.4 \%$ were males. The average follow-up interval was $2.00(1.41)$ years. $95.2 \%$ of patients had successful fusion across all levels. The rate of radiographic non-union was $1.2 \%$ of total segments. The rate of revision surgery was $2.0 \%$. Indications for revision included persistent stenosis/symptoms $(1.4 \%, n=2)$, and pseudoarthrosis $(0.7 \%, n=1)$. The average length to revision was 291 (165) days. The patients with persistent symptoms were treated with posterior laminoplasty and the one patient with pseudoarthrosis was treated with a posterior fusion at the non-fused segment.

Conclusion: Stand-alone ACDF can be performed with good long term rates of fusion without the need for posterior supplementation. Rates of asymptomatic non-union and symptomatic pseudoarthrosis necessitating revision surgery were lower than previously reported. This is the largest series to examine long-term radiographic outcomes in four- and five-level ACDF to date. 


\title{
4:15 - 4:20 pm
}

\section{Characterizing Cortical Plasticity of Patient with Cervical Spondylosis Associated with Neck Pain}

\author{
Chencai Wang; Langston T. Holly MD; Azim laiwalla; Benjamin Ellingson; Noriko Salamon
}

Introduction: Cervical spondylosis (CS) can cause neurological deterioration and cortical atrophy, and cerebral reorganization will be adapted through cortical plasticity to maintain residual motor function. While the majority of focus has been on neurological deficits as a result of spinal cord changes, neck pain has been largely understudied in CS patients.

Methods: 26 CS patients and 32 healthy volunteers (HCs) underwent prospective, observational analysis. Voxel-wise association of DTI measurements and fiber density (FD) with neck pain, measured by the neck disability index (NDI), was assessed in areas of the brain responsible for motor control and pain modulation. Significance was set at $p<0.05$.

Results: The FD difference demonstrated that CS patients have a significant reduction (> 30\%) in the number of fiber tracts connecting the thalamus and primary sensory cortex in comparison to HCs. In addition, a negative correlation between FA values and NDI score was observed in regions, including the basal ganglia, the cerebral and cerebellar peduncles, and fibers along the corticospinal tracts - demonstrating cortical atrophy of primary motor and sensory cortex in CS patients. However, elevated FA along the right posterior corona radiata $(R 2=0.2496, p=0.0094)$, and an increased functional connectivity at the superior frontal gyrus $(R 2=0.2608, p<0.0001)$ and pre- and post-central gyri $(R 2=0.2729, p<0.0001)$ were observed to be associated with worsening neck pain, indicating the presence of cortical reorganization for the preservation of neurological function. Such compensatory mechanisms were further confirmed by higher MD values associated with lower NDI score in regions localized near thalamus, and along fibers projecting to internal capsule, and somatosensory processing areas..

Conclusion: Significant cortical microstructural reorganization occurs in CS patients, particularly associated with sensorimotor regulation and coordination and pain processing and modulation. 
Friday, March 6, 2020

Kuntz Scholar Oral Abstract Presentations

Concurrent Session 3

3:15 - 3:20 pm

Does Spinopelvic Parameters Matter in Short-Segment Posterior Lumbar Spinal Fusion? A MetaAnalysis

Lee Onn Chieng; Mick J. Perez-Cruet MD, MS

Introduction: Restoration of sagittal balance has been associated with better clinical outcome in spinal deformity.However its implications on short-segment lumbar spinal fusion for degenerative spine disease remains unknown.In this study wesoughtto determine the association between adjacent segment disease(ASD) and spinopelvic parameters in short-segement posterior lumbar spinal fusion.

Methods: With adherence to PRISMA guideline, comprehensive database searches were conducted to identify studies that evaluate ASD with spinopelvic parameters in short-segment lumbar fusion.ASD was defined as radiographic and symptomatic axial pain or radiculopathy at adjacent level following index surgery. Independent reviewers extracted data and assessed the outcome.A meta-analysis using random effect weight mean difference model(WMD) was constructed.

Results: A total of 13 studies comprising of 1716 patients underwent 1-3 levels posterior lumbar fusion for degenerative spondylisthesis and spinal stenosis. Of these, 439 patients developed ASD following index surgery while the rest of the patients serve as control. Both groups had comparable basic demographic features in term of age,gender,and BMI. Preoperatively, there were significantly higher pelvic incidence-lumbar lordosis(PI-LL) mistmatch in ASD group(WMD 5.1, 95\% Cl 1.4-8.8; $p=0.007$ ) and lower sacral slope(WMD -1.8, $95 \% \mathrm{Cl}-3.6$ to $0.02 ; p=0.05)$. Interestingly, preop lumbar lordosis (LL) was not observed to be associated with ASD along with pelvic tilt(PT), pelvic incidence(PI), sagittal vertical axis(SVA). However postoperatively, significant loss of LL was then observed in ASD group(WMD $-5.7,95 \% \mathrm{Cl}-8.8$ to $-2.6 ; p=0.0003$ ) along with much higher PI-LL mismatch(WMD 10.1, 95\% Cl 5.6 to 14.6).A subgroup analysis of patients with ASD who eventually treated with surgery was again revealing significant pre- \&post-op PI-LL mismatch>10.

Conclusion: This study demostrated that the relation of pelvis to spine is critical in treating patients with degenerative lumbar spine disease.Failure to correct or progression of PI-LL mismatch were significantly associated with ASD with posterior lumbar spinal fusion even in short-segment constructs. 


\section{3:25 - 3:30 pm}

\section{Multiple Rod Constructs and use of BMP2 Results in Lower Rod Fracture Following Lumbar Pedicle Subtraction Osteotomy}

Darryl Lau; Qiunan Lyu; Vedat Deviren MD; Alexander F. Haddad BS; Christopher P. Ames MD

Introduction: Incidences of rod fracture (RF) remains high following lumbar PSO. Various rod constructs (RC) are used in attempt to solve this problem.

Methods: A retrospective review of ASD patients who underwent lumbar PSO from 2007 to 2017 was performed. Minimum follow-up was 2-years. Three RC were compared: standard (2 main rods), satellite (2 main rods with satellite rods), and nested ( 2 main rods and 2 short rods spanning osteotomy site). Multivariate analysis was used.

Results: 141 patients were included: 55 standard, 23 satellite, and 63 nested. Mean age was 64.9 years and $34.8 \%$ were male. Radiographic preoperative and postoperative results were: SVA (11.0 vs. $3.9 \mathrm{~cm})$, LL (28.5 vs. 57.1 degrees), PT (30.6 vs. 21.0 degrees), and PI (61.5 vs. 60.0 degrees). Time to RF was 12.4 months. Overall RF rate was $22.7 \%$ (bilateral in $5.0 \%)$. Standard-RC had significantly higher rate of bilateral RF $(35.0 \%$ vs. $0.0 \%$ vs. $0.0 \%, p=0.021$ ). Pseudarthrosis and reoperation rate was $20.1 \%$ and $17.7 \%$. Standard-RC experienced significantly higher rate of RF (36.4\% vs. $13.0 \%$ vs. $14.3 \%, p=0.009)$, pseudarthrosis $(34.5 \%$ vs. $8.7 \%$ vs. $12.7 \%, p=0.004)$, and reoperation ( $30.9 \%$ vs. $4.3 \%$ vs. $11.3 \%, p=0.003)$ compared to satellite-RC and nested-RC. Satellite-RC(OR 0.21, p=0.016), nested-RC (OR 0.24, p=0.003), and BMP-2(OR 0.29, $p=0.006$ )were independently associated with lower odds of RF.

Conclusion: Multiple rods and BMP-2 are able to decrease rod-related complications following lumbar PSO. Satellite-RC and nested-RC are able to decrease risk for RF, pseudarthrosis and reoperation. 
3:35 - 3:40 pm

\section{High Radiation Burden of Minimally Invasive Approaches to Adult Spinal Deformity}

Jakub Godzik MD, MSc; Gregory M. Mundis; Celene Bardales Mulholland MD, MPH; Corey T. Walker MD; Jay D. Turner MD, PhD; Juan S. Uribe MD, FAANS; Robert Eastlack MD

Introduction: Minimally invasive spine surgery relies on image guidance, including intraoperative fluoroscopy. As such, the subject of radiation exposure to the patient and surgeon is of concern.

Methods: Patients with ASD from two institutions undergoing cMIS were identified using a prospectively collected database. Inclusion criteria were: coronal cobb (CC) $\geq 20$, sagittal vertical axis (SVA) $\geq 5 \mathrm{~cm}$, pelvic tilt $(\mathrm{PT}) \geq 25^{\circ}$, or thoracic kyphosis (TK) $\geq 60^{\circ}$, undergoing cMIS with more $\geq 3$ intervertebral levels fused, and use of percutaneous screws. Fluoroscopy time (FT) and $\mathrm{mGy}$ for all patients undergoing cMIS was abstracted from the medical records; emission data were compared to risk thresholds based published by the US Nuclear Commission (5 mGy).

Results: 52 patients underwent cMIS deformity correction. Average age was $70 \pm 9$ years, $41 \%$ were female with average BMI 29.4 \pm 5.4 and bone mineral density T-score of-1.6 \pm 0.8 (femur) and $1.4 \pm 0.6$ (spine). Mean preoperative SVA was $9.1 \pm 12 \mathrm{~cm}$, with preoperative $\mathrm{PI}-\mathrm{LL}$ of $14 \pm 14^{\circ}$. On average, patients underwent a $5 \pm 1$ level fusion, with $3 \pm 1$ levels of lateral interbody fusion (LLIF); $40 \%$ of cases were revisions. Mean total FT was $223 \pm 155$ seconds, with mean radiation emission of $179 \pm 191 \mathrm{mGy}(33-1038 \mathrm{mGy})$; the average emission dose exceeded the annual risk threshold 36 times. Radiation dose did not appear to be associated with age $(p=0.81)$, number of levels ( $p=0.37)$, number of LLIF's $(p=0.9)$, sagittal imbalance $(p=0.09)$, and coronal imbalance $(p=0.05)$. The greatest association was with spine T-score $(p=0.004)$.

Conclusion: Radiation emission during cMIS surgery is unsustainably high. It is imperative that we consider and investigate strategies to reduce the radiation burden when treating ASD with cMIS techniques. Special attention needs to be placed on patients with high BMD and appropriate adjustments made to fluoroscope settings to minimize emission. 
Spine Summit Meeting Abstracts, May 2021

3:45 - 3:50 pm

Impact of Opioid Prescribing Guidelines on Postoperative Narcotic Prescriptions Following Elective Spine Surgery

William E. Krauss MD; Elizabeth B. Habermann PhD; Anshit Goyal MBBS; Mohammed A. Alvi MD; Daniel Ubl; Daniel Whipple; Amy E. Glasgow MS; Halena Gazelka; Mohamad Bydon MD

Introduction: With a dramatic rise in prescription opioid use over the last decade, it is imperative to understand post-surgical prescribing patterns and their impact on persistent opioid use. Over-prescribing may contribute to the opioid epidemic due to risk of abuse and diversion.

Methods: Patients undergoing elective cervical or lumbar spine surgery at our institution between 2017-2018 were identified. Procedure-specific opioid prescribing guidelines to limit postoperative prescribing were enacted in January 2018. The cohort was stratified based on whether surgery was performed prior or following guideline implementation. Discharge opioid prescriptions in morphine milliequivalents (MMEs), and the proportion of patients who required a refill within 30 days of discharge as well as the proportion of patients with guideline compliant prescriptions were compared in the two subgroups.

Results: A total of 1,194 patients were identified (cervical: 316; lumbar: 878) with 569 (47.7\%) patients from the pre-guideline period. Guideline adherence was found to be excellent (cervical: $92 \%$, lumbar: $94 \%$ ). Following implementation of the guidelines, fewer patients were discharged with a postoperative opioid prescription (cervical: $90.8 \%$ vs $82.33 \%, p=0.028$; lumbar: $93.3 \%$ vs $81.6 \%, p<0.001$ ). At the same time, the average postoperative opioid prescription was found to decrease significantly (cervical: 343 vs 266 MMEs, p=0.003; lumbar: 323 vs 207 MMEs, p<0.001). Guideline implementation also did not significantly impact the proportion of patients requiring refill prescriptions within 30 days of discharge (cervical: $28.3 \%$ vs $21.7 \%$, $p=0.12$; lumbar: $23.01 \%$ vs $20.0 \%, p=0.27$ ).

Conclusion: Provider-aimed interventions such as implementation of institutional procedure-specific prescribing guidelines may have the potential to significantly reduce postoperative opioid prescriptions following spine surgery without significant impact on the need for refill prescriptions for pain control. 


\section{3:55 - 4:00 pm}

\section{Patients with Bilateral Sacroiliac Joint Vacuum Sign have a Change in Pelvic Incidence between Supine and Standing}

Jeremy L. Fogelson MD; Anthony L. Mikula MD; Soliman Oushy MD; Zachariah Pinter; Pierce Peters; David W. Polly MD; Benjamin D. Elder MD, PhD

Introduction: Pelvic incidence (PI) is an increasingly utilized spinopelvic parameter in the evaluation of spinal deformity patients and is thought to be a fixed parameter.

Methods: A retrospective chart review identified patients with a standing x-ray, supine x-ray, and CT encompassing the SI joints within six months. Patients were grouped according to their SI joints having either no vacuum sign, unilateral vacuum sign, or bilateral vacuum sign. PI was measured by two independent reviewers.

Results: Seventy three patients were identified with an average age of 66 years and a BMI of 30 . Patients with bilateral $\mathrm{SI}$ joint vacuum sign $(\mathrm{N}=27)$ had an average absolute change in $\mathrm{PI}$ of 7.2 degrees $(p$-value $=0.045)$ between the standing and supine position compared to unilateral $\mathrm{SI}$ joint vacuum sign $(\mathrm{N}=20)$ patients who changed 5.2 degrees ( $\mathrm{p}$-value $=0.25$ ) and patients without an $\mathrm{SI}$ joint vacuum sign $(\mathrm{N}=26)$ who changed 4.1 degrees $(p$-value $=0.90)$. Analysis of variance Tukey test showed a statistically significant difference in the change in PI between the bilateral $\mathrm{SI}$ joint vacuum sign patients and those without an $\mathrm{SI}$ joint vacuum sign ( $p$ value $=0.023$ ). Intraclass correlation coefficient between the two reviewers was 0.97 for standing PI and 0.96 for supine PI ( $p$-values <0.0001).

Conclusion: Patients with bilateral SI joint vacuum sign had a change in PI suggesting that there may be substantial motion across the SI joint between the standing and supine position. 


\section{4:05 - 4:10 pm}

\section{Patient Reported Outcomes for Lumbar Spinal Stenosis: Comparison of a Universal and Multitier Health Care System}

Oliver GS Ayling MD, MSc; Tamir Ailon MD, MPH; Nicolas Dea MD, MSc, FRCSC; Raphaele Charest-Morin; Charles Fisher MD

Introduction: Canada has a government funded universal health care systemandaccess to spinal surgeons requires a referral by general practitioners. The United States utilizes a combined public and private payer system where patients may directly access specialists.

Methods: Surgical lumbar spinal stenosis patients treated in Canada that were enrolled in the Canadian Spine Outcome Research Network (CSORN) prospective multicenter registry were compared with the surgical cohort enrolled in the Spine Patients Outcome Research Trial (SPORT) study.

Results: There were 432 and 278 patients in the CSORN and SPORT cohorts. Patients in the CSORN cohort were older (66.8 \pm 10.9 vs. $64.3 \pm 12.5, p=0.017)$, had a higher rate of smoking $(16.7 \%$ vs. $8.9 \%, p=0.0035)$, and were less likely to be employed $(29.7 \%$ vs. $34.2 \%, p=0.043)$. The SPORT cohort had a lower baseline physical component score of the $(33.2 \pm 8.4$ vs. $28.6 \pm 7.5, p<0.01)$. CSORN had a higher proportion of patients with a symptom duration greater than 6 months $(92.3 \%$ vs. $58.3 \%, p<0.0001)$. The CSORN cohort demonstrated significantly greater rates of satisfaction after surgery at 3 months $(89.2 \%$ vs. $60.4 \%, p=0.003)$ and 1 year $(86.8 \%$ vs. $62.6 \%, p<0.001)$.

Conclusion: Patients undergoing surgical treatment for lumbar spinal stenosis in Canada (CSORN cohort) reported higher rates of satisfaction at 3 months and 1 year post-operatively compared to the United States cohort (SPORT) despite having longer durations of symptoms prior to surgery. 


\section{4:15 - 4:20 pm}

\section{Development and Validation of a Prediction Score for Poor Postoperative Pain Control Following Elective Spine Surgery}

Michael M.H. Yang MD, M.Biotech, MSc; Jay Riva-Cambrin MD; Jonathan Cunningham; Nathalie Jette; Tolulope Sajobi; Steven Casha MD, PhD

Introduction: Postoperative pain is often poorly controlled following spine surgery. The ability to identify these patients before surgery may be useful to facilitate the development of personalized clinical-care pathways to improve postoperative pain management.

Methods: Adult patients were consecutively enrolled through the Canadian Spine Outcomes and Research Network registry and were included if they underwent elective cervical or thoracolumbar spine surgery. The outcome was poor pain control defined as the mean numeric-rating-scale for pain $>4$ in the first 24-hours after surgery. A split-sample design was used to develop and validate the prediction model. The prediction model was transformed into a risk-based score and simplified to a three-tier score to maximize clinical utility.

Results: Of 1,300 patients, $57 \%$ had poorly controlled pain. Seven significant predictors were associated with poor pain control: younger age, female sex, preoperative daily opioid medication use, higher preoperative neck/back pain intensity, higher PHQ-9 depression score, $\geq 3$ motion segment operation, and fusion surgery. The model was discriminative (c-statistics 0.74 [95\% Cl=0.71-0.77]) and accurate (Hosmer-Lemeshow goodness-of-fit, $p=0.99$ ) at predicting the outcome. Patients classified to low-, high-, and extreme-risk groups by the score had $32 \%, 63 \%$, and $85 \%$ predicted-probability of developing poor pain control, respectively. This closely mirrored the observed-probability of $37 \%, 62 \%$, and $81 \%$ in the same risk-groups for poor pain control in the validation cohort.

Conclusion: This validated prediction score based on seven easily acquired characteristics accurately predicted the probability of developing poor pain control after spine surgery. 
Friday, March 6, 2020

Minimally Invasive/Motion Preservation/Spondylolisthesis

Abstract Breakout Session

3:15 - 3:17 pm

Variability in Recommendations for Treating Grade 1 Spondylolisthesis, in 114 Patients Evaluated by 28 Spine Surgeons

Eric N. Momin MD; Bradley T. Schmidt MD; Scott Hetzel MS; Frederick G. Barker MD, FACS; Zoher Ghogawala MD, FACS; Daniel K. Resnick MD, MS

Introduction: The role of fusion in symptomatic lumbar spondylolisthesis remains debated. We hypothesize that there is significant variability in whether a fusion is recommended for grade 1 spondylolisthesis, and that this is due to both patient and surgeon factors.

Methods: Our data originate from one arm of the SLIP II study in which 28 senior surgeons evaluated 114 patient cases, and made treatment recommendations for each case. Responses ranged from No surgery (score 1) to Instrumented Interbody Fusion (score 6).

Results: Surgeon demographics spanned a variety of geographies, practice type, training, and specialty. Overall, 1463 treatment recommendations were given. The variability in recommendations was visualized with a heatmap. On average, fusion was recommended $58.5 \%$ of the time. Overall agreement was low, and perfect agreement on the need for fusion was seen in only $24(21.1 \%)$ of patients. Kappa statistic for agreement on fusion was $0.378(95 \% \mathrm{Cl} 0.324$ to 0.432$)$. The average patient received a treatment recommendation score of 4.2, however, the average standard deviation was 1.4 indicating a high variability of surgical recommendations.

Conclusion: Even among expert spine surgeons, there is significant disagreement in whether to perform fusion for grade 1 spondylolisthesis. Individual patients in this study received a wide variety of treatment recommendations. Additionally, some patients tended to receive more invasive surgical recommendations than others. We suspect that the population of patients described by the diagnosis of "grade 1 spondylolisthesis" is a heterogeneous group in which there may not be one optimal treatment strategy. 
3:17 - 3:19 pm

\section{Machine Learning Segmentation of Lumbar Spinal Canal in Lumbar Stenosis}

Azim laiwalla; Bilwaj K. Gaonkar PhD; Bryan Yoo; Banafsheh Salehi; Mark A. Attiah MD, MS, MBE; Wi Jin Kim; Bayard Wilson BA; Joel S. Beckett MD, MHS; Luke Macyszyn MD, MA

Introduction: Lumbar stenosis (LS) is a debilitating condition, occurring in up to $77.9 \%$ of adults over 40 , and is the most frequent cause of spinal surgery in patients older than 65. Osteocartilaginous and ligamentous degeneration and hypertrophy causes progressive canal constriction and neurovascular compromise, resulting in lower back pain, radicular lower extremity pain, and neurogenic claudication. As the degree of canal constriction leading to symptomatic LS is unclear, methods to assess canal area are of significant interest.

Methods: Hybrid machine learning algorithms were trained to segment lumbar spinal canals at each level (L1L5) on 50 PACS-acquired axial MR images of LS patients. These segmentations were compared with two sets of expert-generated segmentations. Dice scores, the Hausdorff distances, and average surface distance metrics were used to compare overlaps between automatic and expert-generated segmentations, as well as interrater segmentation.

Results: Machine-generated segmentations $\left(\mathrm{M}_{\mathrm{A}}\right)$ were qualitatively similar to both expert-generated segmentations $\left(\mathrm{M}_{E 1}, \mathrm{M}_{\mathrm{E} 2}\right)$ despite irregular and inconsistent lumbar canal shapes. Furthermore, machine- and expert- generated segmentations were quantitively comparable as indicated by dice scores (MA vs. ME $0.85+/-0.17, \mathrm{MA}$ vs. $\mathrm{ME}_{2}: 0.84+/-0.18$ ), the Hausdorff distance (MA vs. $\mathrm{ME}_{1}: 17.0 \mathrm{~mm}+/-17.8, \mathrm{MA}$ vs. $\mathrm{ME}_{2}$ : $15.8 \mathrm{~mm}+/-20.4$ ), and average surface distance (MA vs. $M_{E} 1: 1.1 \mathrm{~mm}+/-4.9, M_{A}$ vs. $M_{2}: 1.1 \mathrm{~mm}+/-4.9$ ) metrics. Further, these metrics are in the same range as variations between the expert raters (dice scores: $0.93+/-0.07$, the Hausdorff distances: $12.0 \mathrm{~mm}+/-20.0$, average surface distances: $0.17 \mathrm{~mm}+/-0.46$ ).

Conclusion: We have trained a novel hybrid machine learning algorithm to automatically segment lumbar spinal canals in LS patients. These automatic segmentations are both qualitatively and quantitively comparable to expert-generated segmentations. 
3:19 - 3:21 pm

\section{Full Endoscopic Posterior Cervical Foraminotomy: Early Experience and Comparison with Open Surgery}

Masahiko Akiyama MD, DMSc; Hisashi Koga

Introduction: Posterior cervical foraminotomy is one of the standard surgeries for cervical spondylotic radiculopathy and full endoscopic application have recently applied instead of classical open surgery.

Methods: Six cases of one level FEPCF were carried out during March and August of 2019, and 6 cases of previously performed one level OPFC were included for comparison in this study. Operating time, foraminotomy area calculating from postoperative reconstructed 3D-CT scan, improvement of Oswestry neck disability index (ONDI) at 1 month after surgery, and use of postoperative PRN medication were compared. Mann-Whitneys $u$ test was used for statistical analysis.

Results: Operating time of FEPCF and OPFC was $73.7 \mathrm{~min}$ and $86.7 \mathrm{~min}$ respectively and not statistically different $(p=0.52)$. Foraminotomy area of FEPCF was $52.33 \mathrm{~cm} 2$, significantly smaller than that of OPFC of $96.18 \mathrm{~cm} 2$ ( $p=0.037$ ). Improvement of ONDI 1 month after surgery was not significantly different $(50.4 \%$ for FEPCF and $41.0 \%$ for OPCF $p=0.72$ ). Use of postoperarive PRN pain medication was significantly less in FEPCF, 0.67 times as compared with OPCF, 6.83 times $(p=0.0056)$.

Conclusion: One level FEPCF is as effective as OPCF in terms of radiculopathy related symptoms and requires similar operating time as OPCF. In addition, FEPCF significantly reduces foraminotomy area requires for nerve root decompression, and use of postoperative pain medication compared with OPCF. FEPCF is technically feasible and considered as one of the minimum invasive treatment options for cervical spondylotic radiculopathy. 


\section{3:21 - 3:23 pm}

\section{Transforaminal Endoscopic Lumbar Approaches with Radiofrequency Nerve Stimulation and Continuous Electromyography}

Ibrahim Hussain; Benjamin I. Rapoport; Katie Krause; Gregory Kinney; Christoph Hofstetter; Eric H. Elowitz MD

Introduction: Transforaminal endoscopic lumbar approaches for discectomy or foraminotomy involve working in Kambins triangle. These procedures are performed on awake patients or under general anesthesia with continuous electromyography (EMG). Potential morbidity of this approach includes injury to the exiting and traversing nerve roots since substantial dissection or cauterization of overlying tissues are required for visualization.

Methods: 30 consecutive patients undergoing transforaminal endoscopic lumbar approaches for discectomies $(73.3 \%)$, foraminal stenosis $(23.3 \%)$, or lateral recess stenosis $(3.3 \%)$. were enrolled. Demographic, operative, and outcomes data were collected.

Results: The average age was 61.4 years and the $L 4 / 5$ segment was most commonly treated (65.6\%). Electrophysiological mapping of the exiting nerve root was attempted in 28 patients with an average stimulation threshold of $8.6 \pm 0.9 \mathrm{~mA}$. Mapping of the traversing nerve root was attempted in 12 patients with an average stimulation threshold of $6.0 \pm 0.8 \mathrm{~mA}$. There were no instances of new postoperative sensorimotor deficits or dysesthesia. These findings persisted through a mean and median follow-up of 294 days and 165 days, respectively. No patient required subsequent lumbar surgery.

Conclusion: Our modified instrumentation and technique allows for accurate identification of the exiting and traversing nerve roots with minimal changes to the workflow of transforaminal endoscopic lumbar approaches. Modification of a bipolar radiofrequency device connection arrangement is simple, inexpensive, and reusable. In our study, no patients developed injury or pain related to nerve root dysfunction. 
3:23 - 3:25 pm

\section{Minimum Clinically Important Difference in Outcomes after Surgery for Grade 1 Spondylolisthesis: A QOD Analysis}

Mohamad Bydon MD; Praveen V. Mummaneni MD; John Knightly; Anshit Goyal MBBS; Mohammed A. Alvi MD; Andrew Kai-Hong Chan MD; Jian Guan MD; Steven D. Glassman ; Kevin T. Foley MD, FACS, FAANS; Jonathan Slotkin MD; Eric A. Potts MD; Mark E. Shaffrey MD, FAANS, FACS; Christopher I. Shaffrey MD, FACS; Regis W. Haid; Kaiming Fu; Michael Y. Wang MD, FAANS; Paul Park MD; Anthony L. Asher MD, FACS; Erica F. Bisson MD, MPH, FAANS

Introduction: Minimum Clinically Important Difference(MCID) and substantial clinical difference(SCD) in patient reported outcomes (PROs) are considered standards for determining effectiveness and measurement of patient satisfaction in response to intervention.

Methods: We queried the QOD for patients with grade 1 lumbar degenerative spondylolisthesis undergoing a surgical intervention between July 2014 and June 2016. Both anchor-based(NASS satisfaction scale) and distribution-based methods were used to determine MCID in ODI, EQ-5D, Numeric Rating Scale-Back Pain(NRS-BP) and Numeric Rating Scale-Leg Pain(NRS-LP) at 1 year and 2 years following surgery. SCD was determined using an anchor-based approach (ROC analysis, change difference). NASS satisfaction=1 indicated SCD while NASS satisfaction=1/2 indicated MCID.

Results: There were 602 patients with available baseline PROs eligible for inclusion in the analysis. The calculated MCID values were highly variable at both 1 year(ODI:3.43-20.9; EQ5D:0.045-0.17; NRS-BP:0.56-4; NRS-LP:0.57-5) and 2 years(ODI:3.43-17.9; EQ5D:0.045-0.19; NRS-BP:0.56-4; NRS-LP:0.57-3). SCD values at 1 year were ODI: 23.8, EQ5D: 0.165; NRS-BP: 4 and NRS-LP: 5; at 2 years: ODI:30, EQ5D: 0.165; NRSBP: 4 and NRS-LP: 5 points and were substantially higher than the change difference seen in satisfied vs nonsatisfied patients. The MDC method yielded values higher than the measurement error and was generally closer to the change difference in satisfied vs non-satisfied patients. Subgroup analyses for decompression alone vs fusion showed similar values for MCID and SCD for ODI, however values were higher for EQ5D and NRSBP in patients undergoing fusion while values for NRS-LP were higher in patients undergoing decompression alone.

Conclusion: MCID determination for grade I spondylolisthesis is highly variable depending on the measurement technique. MDC method is statistically appropriate for MCID calculations. SCD in PRO scores is higher than difference in average improvement between patients considered to have achieved substantial benefit versus those who do not. 


\title{
3:25 - 3:27 pm
}

\section{Patient Satisfaction after MIS Lumbar Fusion: Comparison of Three Interbody Techniques}

\author{
W. H. Andrew Ryu BSc, MSc, MTM, MD; Alia Rathore; Brian T. David PhD; Richard G. Fessler MD, PhD
}

Introduction: There has been a growing emphasis in patient satisfaction after elective spine surgery as part of evaluating operative outcomes. With expansion of different minimally invasive surgery (MIS) interbody techniques, surgeons have examined the relative clinical and radiographic outcomes of these techniques. However, whether patient satisfaction differ depending on the type of interbody fusion utilized remains uncertain.

Methods: Retrospective chart review was performed on patients who underwent MIS lumbar interbody fusion between 2015-2019 involving up to 3 spinal levels. Inclusion criteria were age>18, degenerative spine pathology, and completed patient reported outcome (PRO) surveys postoperatively with minimum of 6-month follow-up. Patient satisfaction was rated using visual analog scale out of 50 with 0 representing "delighted" and 50 representing "not satisfied". Patient demographics, clinical data, and PRO scores were compared between the open and MIS groups.

Results: In total, 140 patients met the inclusion criteria (average age 62.1 years; 73 females: 67 males) consisting of 29 anterior lumbar interbody fusion (ALIF), 26 lateral lumbar interbody fusion (LLIF), and 85 transforaminal lumbar interbody fusion (TLIF). Majority of the cases (105/140) were single level fusion. At postoperative follow-up, overall satisfaction score was similar between the three procedures (ALIF 12.4 vs. LLIF 13.0 vs. TLIF 13.9; $p>0.05$ ). Similarly, patient satisfaction scores on back pain improvement and leg pain improvement were comparable between the three groups (ALIF 20.0 vs. LLIF 16.3 vs. TLIF 16.4; $p>0.05 ;$ ALIF 16.4 vs. LLIF 17.3 vs. TLIF 13.8; $p>0.05$, respectively).

Conclusion: Patients who underwent MIS lumbar interbody fusion reported satisfaction in their back pain, leg pain, and overall surgical outcome. This positive outcome was seen in all the three commonly utilized interbody fusion techniques. 
3:27 - 3:29 pm

\section{Radiographic Parameters of Listhesis are Not Associated with Health Status in Grade 1 Degenerative Spondylolisthesis}

Joseph Laratta; Aidin Kashigar; Erica F. Bisson MD, MPH, FAANS; Leah Carreon; Andrew Y. Yew MD; Tino Mkorombindo; Steven D. Glassman

Introduction: Slip magnitude and presence of motion are utilized in surgical planning for degenerative spondylolisthesis. Fusion is considered in patients with higher slip magnitude or instability.

Methods: From the Quality Outcomes Database, patients who had fusion for grade 1 degenerative spondylolisthesis with complete pre-op and one-year post-op Patient Reported Outcomes (PROs) were identified. The magnitude of slip and presence of motion were measured on pre-op MRI, upright neutral, flexion, and extension x-rays. Pre-op and one-year post-op PROs including Back Pain (BP, 0-10), Leg Pain (LP, 0-10), Oswestry Disability Index (ODI) and EQ-5D were analyzed.

Results: Seventy-nine patients were identified. Mean age was 60.7 years and there were 46 females (58\%). Patients were categorized based on upright slip into 3 groups: $<5 \mathrm{~mm}, 5-7 \mathrm{~mm}$, and $>7 \mathrm{~mm}$ slip. Motion was defined as $>3 \mathrm{~mm}$ slip difference between flexion and extension films and supine MRI-upright neutral $x$-rays. No significant differences were identified in PROs at baseline or at one-year post-op follow-up between the groups $(p>0.05)$.

Conclusion: Slip magnitude and presence of motion were not associated with pre-op or 12 month post-op PROs for patients with grade 1 degenerative spondylolisthesis. 


\section{3:29 - 3:31 pm}

\section{Fusion Versus Decompression Alone for Lumbar Spondylolisthesis: Impact on the 10 Sub-Items of Oswestry Disability Index}

Andrew Kai-Hong Chan MD; Erica F. Bisson MD, MPH, FAANS; Mohamad Bydon MD; Kevin T. Foley MD, FACS, FAANS; Steven D. Glassman; Christopher I. Shaffrey MD, FACS; Eric A. Potts MD; Mark E. Shaffrey MD, FAANS, FACS; Domagoj Coric MD; John J. Knightly MD; Paul Park MD; Michael Y. Wang MD, FAANS; Kai-Ming G. Fu MD, PhD; Jonathan Slotkin MD; Anthony L. Asher MD, FACS; Michael S. Virk MD, PhD; Panagiotis Kerezoudis ; Mohammed A. Alvi MD; Jian Guan MD; Regis W. Haid; Praveen V. Mummaneni MD

Introduction: The impact of fusion procedures on lumbar spondylolisthesis has not been investigated as it pertains to the individual components of the Oswestry Disability Index (ODI). Such study may offer insights into the specific benefit fusion may hold with regards to different domains of disability.

Methods: Prospectively-collected data from the Quality Outcomes Database (QOD) registry was analyzed. 608 patients underwent elective single-segment spine surgery for degenerative grade 1 lumbar spondylolisthesis. Responses for the 10 individual items of the ODI, which focus on disability in the domains of pain intensity, self care, heavy-weight lifting, walking, sitting, standing, sleeping, sexual activity, social activity, and ability to travel, respectively, were collected at baseline and 24 months. Multivariable methods were utilized to assess the impact of fusion on each ODI sub-item.

Results: 468 (77.0\%) patients underwent fusion; 140 (23.0\%) underwent decompression alone. Fusions were younger ( 59.9 vs. 69.6 years, $p<0.001)$ and had a greater proportion with preoperative back pain $(86.1 \%$ vs. $52.9 \%, p<0.001)$. At 24 months, both cohorts improved significantly for each ODI sub-item ( $p<0.05$, all subitems, for each cohort respectively). Adjusting for baseline differences, fusion-compared to decompression alone-was associated with superior outcomes for pain intensity $(\beta=-0.4, p<0.001)$, heavy-weight lifting ( $\beta=-$ $0.4, p=0.02)$, walking $(\beta=-0.5, p<0.001)$, standing $(\beta=-0.5, p=0.002)$, sexual activity $(\beta=-0.3, p=0.01)$ social activity $(\beta=-0.5, p<0.001)$, and ability to travel $(\beta=-0.4, p=0.002)$. However, there were no significant associations between fusion and self care (e.g., washing, dressing), sitting, and sleeping ( $p>0.05)$.

Conclusion: Fusions were associated with superior outcomes for intensity of back pain, ability to lift heavy weights, walk, stand, travel, and participate in both sexual and social activities. These results may help to cater the surgical decision-making process to an individual patients chief complaint. 
3:31 - 3:33 pm

\section{Multi-level MIS Transforaminal Lumbar Interbody Fusion in the Elderly: A Predictive Modeling Comparative Cohort}

Chad F. Claus DO; Evan J. Lytle DO; Doris Tong; Matthew Bahoura BA; Lucas Garmo; Elise J. Yoon BS; Jake Jasinski DO; Ascher Kaufmann; Boyd F. Richards DO; Teck Mun Soo

Introduction: The number of elderly patients undergoing spinal fusion is rising. Spinal surgery in the elderly is considered high risk with high rates of complications. However, perioperative and postoperative complications in the elderly undergoing multi-level minimally invasive transforaminal lumbar interbody fusion (MIS TLIF) is not known.

Methods: A retrospective analysis was performed on 467 consecutive patients who underwent multi-level MIS TLIF at a single institution from 2013 to 2017. Two cohorts, 70 years or older and 50-69 years old were evaluated by univariate analyses. Multiple logistic regressions with minor and major complication rates as the dependent variables were performed to identify predictors for complication based on previously sited risk factors. A p-value of $\leq 0.025$ was considered significant.

Results: One-hundred-fifty-two elderly and 315 non-elderly patients underwent multi-level MIS TLIFs. The average age was 76.4 years and 60.4 years for the elderly and non-elderly cohorts. We observed 13 major $(8.44 \%) 72$ minor $(47.4 \%)$ complications in the elderly. No difference was noted in complication between the cohorts, except for UTI ( $p=.004)$ and urinary retention $(p=.014)$. There were no myocardial infarctions, hardware complications, visceral, vascular, neural injuries, or death. Length of stay, comorbidity, and length of surgery was predictive of major and minor complications.

Conclusion: Elderly may undergo multi-level MIS TLIF with comparable complication rates. Age was not a predictor of complications. Rather, attention should focus on the evaluation of comorbidity and limiting operative times. 
3:33 - 3:35 pm

\title{
Preliminary Radiographic Results of the Prone Transpsoas Technique PTP Multi Center
}

\author{
Luiz Pimenta; William R. Taylor MD; Antoine Tomeh; Rodrigo Augusto Amaral MD; Gabriel Pokorny
}

Introduction: The lateral lumbar interbody fusion (LLIF) is a safe and effective technique to treat a vast range of lumbar disorders.

Nevertheless, the LLIF is also burdened by some problems, such as postoperative neurological deficits, small segmental index level lordosis, and the necessity to re-position the patient to instrument posteriorly.

A new approach to the lateral lumbar interbody fusion was devised to solve or reduce some of the problems regarding the lateral approach. Its principal difference from the standard LLIF relies on positioning the patient in a prone decubitus, which might lead to an increase in the intradiscal lordosis.

Methods: A retrospective, multicenter, non-comparative, and non-randomized study was undertaken to evaluate segmental and regional lordosis following prone transpsoas (PTP) approach to LLIF. All patients undergoing prone transpsoas surgery at the involved institutions were included. Patients with low-quality images not allowing the measurement of the required spinopelvic parameters were excluded. Measurements included pre- and post-operative index-level segmental lordosis, lumbar lordosis, pelvic incidence, pelvic tilt.

Results: Thirty-two (32) patients were included in the study, in which 23 underwent single-level, 6 underwent two-level, 2 underwent three-level, and 1 underwent four-level PTP. Mean index-level segmental lordosis increased from $8,7^{\circ}$ to $14,8^{\circ}$ ( $p<0.001$ ), lumbar lordosis (L1-S1) increased from $42,1^{\circ}$ to $45,8^{\circ}(p=0,11)$, although after excluding an outlier value the L1-S1 lordosis results were $41,9^{\circ}$ pre-op to $46,7^{\circ}$ post-op $(p=0,003)$. Twenty-two (22) patients had a pre-op PI-LL mismatch $\geq 10^{\circ}$ or $\leq-10^{\circ}$, while at the postoperative visit, only 12 patients had a mismatch $\geq 10^{\circ}$ or $\leq-10^{\circ}(p=0,01)$.

Conclusion: The prone transpsoas technique is safe and feasible, and it is associated with a significant gain of segmental lordosis and correction of spinopelvic mismatch. 
Spine Summit Meeting Abstracts, May 2021

3:35 - 3:37 pm

\section{Hounsfield Units by Computed Tomography Correlates with Cage Subsidence after Transforaminal Lumbar Interbody Fusion}

Zhuo Xi MD, PhD; Chih-Chang Chang; Pingguo Duan; Sigurd Berven; Praveen V. Mummaneni MD; Dean Chou

Introduction: Cage subsidence can occur after transforaminal lumbar interbody fusion (TLIF). Hounsfield units (HU) from computed tomography (CT) has been correlated with bone density.

Methods: A retrospective study of patients who underwent TLIF at L4-L5 was performed. Patients with cage subsidence were matched 1:1 to controls without cage subsidence on the basis of age and sex. HU values were measured from the preoperative lumbar CT. Cage subsidence was evaluated using lumbar CT at minimum of 1 year post-operatively. HU values were measured from L1 to L5, and each lumbar vertebral body was measured in 4 separate times: the mid-sagittal plane, mid-body axial, near superior endplate axial, and near inferior endplate axial. The mean $\mathrm{HU}$ value of the 4 measurements was used as the vertebral body $\mathrm{HU}$ value.

Results: 60 total patients were included in this study. The mean lumbar HU values were significantly lower in patients with cage subsidence than without $(110.41 \pm 10.08$ vs. $141.58 \pm 10.21 ; P<0.05)$. Because the majority of the TLIFs were at L4-5, the mean L4 HU values were $139.5 \pm 9.47$ in cage subsidence group and $163.25 \pm$ 8.13 in the control group $(P<0.05)$. Similar, The mean L5 HU values for cage subsidence group $(121.29 \pm$ 10.01) were significantly lower than that in control group (171.36 $\pm 11.23, \mathrm{P}<0.01)$.

Conclusion: TLIF cage subsidence correlated with lower HU values by CT, especially at L4 and L5. HU may be a useful marker for pre-operative optimization to decrease subsidence rates. 


\title{
3:37- 3:39 pm
}

\section{Increasing Intraoperative Efficiency in Circumferential Lumbar Surgery: Eliminating the Flip}

\author{
Richard A. Hynes; Ron Lehman; Olumide Aruwajoye; Daniel Refai MD; Russ P. Nockels MD
}

Introduction: Circumferential lumbar surgery (CLS) is desirable to optimize clinical outcomes in select patients. The popularity of oblique and lateral interbody approaches has complicated the decision to perform posterior stabilization with pedicle screws. Surgeons often perform stand alone procedures, stage surgeries, or do not achieve restoration of lumbopelvic realignment. As a result, these approaches are 1) suboptimal, 2) time consuming and 3 ) not cost effective.

Methods: We prospectively enrolled 24 consecutive patients undergoing OLIF and posterior lumbar fusion, and compared them to a matched, cohort of 24 patients undergoing the same procedure but with a standard flip (re-prepping and draping) without the PPS system). All procedures were performed at a single institution and all relevant timepoints recorded.

Results: There were no significant demographic or procedural differences between the study and cohort groups (average age 63, M/F 27/21). The average number of levels fused was 2.25 in both groups. Repositioning times differed significantly between the study and cohort groups $(5.1 \pm 1.5$ min. versus $35.7 \pm 6.0$ min., $p<0.001$, t-test) There were no surgical or positioning complications in either group.

Conclusion: :This novel patient positioning system (PPS) reduced operative repositioning time when performing OLIF and posterior spinal fusion by $86 \%$, resulting in over 30 minutes of decreased operative time. These procedural advances will significantly improve work-flows and optimize spine surgery. 
3:39 - 3:41 pm

\section{Types and Timing of Reoperation Following Surgery for Grade 1 Degenerative Lumbar Spondylolisthesis}

Andrew Kai-Hong Chan MD; Erica F. Bisson MD, MPH, FAANS; Mohamad Bydon MD; Kevin T. Foley MD, FACS, FAANS; Steven D. Glassman; Christopher I. Shaffrey MD, FACS; Eric A. Potts MD; Mark E. Shaffrey MD, FAANS, FACS; Domagoj Coric MD; John J. Knightly MD; Paul Park MD; Michael Y. Wang MD, FAANS; Kai-Ming G. Fu MD, PhD; Jonathan Slotkin MD; Anthony L. Asher MD, FACS; Michael S. Virk MD, PhD; Panagiotis Kerezoudis ; Mohammed A. Alvi MD; Jian Guan MD; Regis W. Haid; Praveen V. Mummaneni MD

Introduction: The comparative durability of decompression and fusion versus decompression-only surgery for lumbar spondylolisthesis remains unclear.

Methods: 608 patients at twelve, top-enrolling sites underwent single-segment spine surgery for grade 1 degenerative lumbar spondylolisthesis. Baseline through 24-month data were collected. Types and timing of reoperations were recorded. Reoperation occurrence was assessed at 30-day, 1-year, 2-year, and 3-year time points.

Results: 468 (77.0\%) patients underwent fusion and 140 (23.0\%) underwent decompression alone. Fusions were younger (59.9 vs. 69.6 years, $p<0.001)$, had higher mean BMI $(30.9$ vs. $28.7, p<0.001)$, and a greater proportion with preoperative back pain $(86.1 \%$ vs. $52.9 \%, p<0.001)$.

The overall reoperation rate was 6.9\%. Reoperation rates were similar (Decompression alone:9.3\% vs. Fusion: $6.2 \%, p=0.21)$. However, there were more reoperations within 30 days for fusions $(p=0.02)$, whereas there were significantly more reoperations from 30 days to 1 year for decompression alone $(p=0.01)$. There were no differences at other time points $(p>0.05)$.

Among decompression-alone reoperations, $53.8 \%$ were revision decompressions and $46.2 \%$ were for fusions. Among fusion reoperations, 3.2\% were for decompression of adjacent segment stenosis, $41.9 \%$ were revision fusions, and $54.8 \%$ were for miscellaneous (SSI, implant revision/removal, hematoma evacuation, wound revision, and spinal cord stimulator).

In multivariable models, reoperations were independently associated with inferior change in Oswestry Disability Index $(\beta=10.1,95 \% \mathrm{Cl}(4.5-15.7), p<0.001)$ and NASS Satisfaction $(\mathrm{OR}=3.1,95 \% \mathrm{Cl}(1.7-5.5), p<0.001)$. In predictor modeling, shorter surgery time was the only factor predictive of reoperation (OR=1.01 per minute shorter, $95 \% \mathrm{Cl}(1.003-1.013), p=0.004)$.

Conclusion: Though reoperation rates are similar between cohorts, the types and timing of reoperations differ. Fusions had a higher rate of reoperation 30 days following surgery, whereas decompression alone was associated with a higher rate between 30 days and 1 year. Reoperations have a negative impact on disability and patient satisfaction. Shorter lengths of surgery were associated with an increased odds of reoperation. 
3:41 - 3:43 pm

\section{Cervical Disc Arthroplasty: Comparison of 4 Semi-Constrained Implants Using FEA}

Yuvi Purushothaman; Davidson Jebaseelan; Hoon Choi MD, MS; Jamie Baisden MD; Narayan Yoganandan

Introduction: CDA differ in design and material properties. This effects there biomechanical properties. We will compare 4 different semi-constrained CDA's at C5-6 in a cervical spine model using FEA.

Methods: 4semi-constrained CDA (Prodisc-C PC, Prestige-LP PLP, Mobi-C MC, and Secure-C SC) were tested in a validated subaxial $\mathrm{C}$-spine FEM using both pure moment at $2 \mathrm{Nm}$ and hybrid moment assessing the ROM, discal pressures, and facet loads at the index, and adjacent rostral and caudal levels.

Results: ROM trends between the 4 CDA's are similar at the rostral adjacent level for both flexion (5.4-6.7\%) and extension (12.5-18,8\%) and caudally at the adjacent level in extension (5.4-7.1\%). Flexion ROM at the adjacent level PLPand MC (11.5 and 11.6\%) are twice that of PC and SC (4.3and 5.9\%).

PLP (M-M)design in unique for ACL loading. PC-has least ACL changes among the $3 \mathrm{M}-\mathrm{P}$ CDA's at both levels and both modes because of no translation. MC-has the greatest ACL change among the 3 M-P CDA's at both levels and both flexion and extension modes, likely due to fixation of the polymer to metal. PLP increases while SC decreases ACL.

Conclusion: This suggests there is an interaction between M-M/M-P interface, the translational level, and additional design features which may explain the flexion ROM and ACL differences between CDA's. The difference in types of constraint between M-P may explain adjacent level kinematics. Secure-C's more rigid attachment of the polymer may be responsible for less PCL changes which may reduce long-term effects such as $\mathrm{HO}$, and facet arthropathies. 


\section{$3: 43-3: 45 \mathrm{pm}$}

\section{Biomimetic 3D-printed Titanium-Alloy Interbody Spacers Demonstrate Uniform Bone Growth over 12 Weeks in an Ovine Model}

Margaret Van Horn; Roland Beard; Bryan W. Cunningham; Ken Mullinix; Brandon Bucklen PhD

Introduction: A primary clinical outcome of interbody fusion surgery is achievement of bony fusion. Biomimetic implants may promote formation of new bone. A uniform rate of bone formation during healing may lead to optimized fusion.

Methods: Eighteen sheep underwent a two-level LLIF using polyetheretherketone (PEEK), titanium-alloy (TAV), or 3D-printed biomimetic TAV (3DP) spacers ( $n=6$ levels per group per time point). Animals were sacrificed at 6 and 12 weeks postoperatively. Cadaver sheep ( $n=4$ levels per group) were implanted with bone graft packed spacers to quantify the initial 0 week time point. Microcomputed tomography quantified bone volume (BV) through the spacer and BGR between time points was analyzed.

Results: The 3DP-treated group demonstrated a more consistent BGR over 12 weeks compared to PEEK and TAV groups. The BGR for the 3DP group was $10.6 \mathrm{~mm}^{3} /$ week and $9.6 \mathrm{~mm}^{3} /$ week from 0 to 6 weeks and 6 to 12 weeks, respectively. Bone growth in PEEK and TAV groups was delayed, evidenced by not surpassing the remodeling phase until after 6 weeks and an inconsistent BGR between early and late time intervals (PEEK: 1.5 and $17.0 \mathrm{~mm}^{3} /$ week, respectively; TAV: -2.1 and $17.7 \mathrm{~mm}^{3} /$ week, respectively).

Conclusion: 3DP biomimetic TAV interbody spacers facilitated uniform bone growth through the spacers over 12 weeks of healing, with lower bone volume observed with PEEK and TAV implants at the 6 week time point. Biomimetic 3DP implants lead to robust intervertebral fusion. 
3:45 - 3:47 pm

\section{Rough Biomimetic Titanium Surface is Associated with Increased Alkaline Phosphatase Activity in Mesenchymal Stem Cells}

Margaret Van Horn; Roland Beard; Brandon Bucklen PhD

Introduction: Osseointegration and implant stability are a primary goal of many orthopaedic surgeries. Implants with biomimetic surface features may stimulate osteoblastic activity and be optimal for bone growth. Subtractive laser-etching allows for controlled, intentionally designed micro-scale features to be applied to surfaces.

Methods: 3D surface roughness $\left(S_{a}\right)$ was characterized using a scanning electron microscope. Cells were cultured for 7 days at a seeding density of 10,000 cells $/ \mathrm{cm}^{2}$ on PEEK, smooth TAV (sTAV), and a roughened, biomimetic TAV (rTAV) substrates ( $n=6$ per group). Cellular proliferation was measured at days 1,3 and 7 . At day 7 cells were lysed and alkaline phosphatase (ALP) activity, an early marker of osteoblastic differentiation, was assayed. ALP activity was normalized to total protein in each lysate sample.

Results: The rTAV substrates with bone-inspired topography had 10x greater $\mathrm{S}_{a}$ than PEEK and sTAV $(36.3 \mu \mathrm{m}, 0.3 \mu \mathrm{m}$, and $0.4 \mu \mathrm{m}$, respectively). Cell proliferation on rTAV was significantly greater than PEEK $(p<0.05)$, and was not significantly different from sTAV $(p>0.05)$. Normalized ALP activity was significantly greater in the $\mathrm{rTAV}$ group $(16.35 \pm 1.80 \mathrm{nmol} / \mu \mathrm{g} \mathrm{min} / \mathrm{mL})$ compared to both PEEK $(6.61 \pm 1.51 \mathrm{nmol} / \mu \mathrm{g}$ $\mathrm{min} / \mathrm{mL})$ and $\operatorname{sTAV}(7.12 \pm 2.01 \mathrm{nmol} / \mathrm{\mu g} \mathrm{min} / \mathrm{mL})(\mathrm{p}<0.05)$.

Conclusion: A roughened biomimetic TAV substrate elicited significantly higher cellular activity compared to PEEK and significantly higher ALP activity than both sTAV and PEEK, suggesting the rTAV substrate facilitated a more osteogenic environment. 


\section{3:47 - 3:49 pm}

\section{Surgical Outcomes of Dynamic Cervical Implants vs Total Disc Replacement and ACDF: A Systematic Review and Meta-Analysis}

Aya Akhras; Waseem Wahood; Atiq Bhatti; Mohammed A. Alvi MD; Anshit Goyal MBBS; Mohamad Bydon MD

Introduction: Dynamic cervical implants $(\mathrm{DCl})$ a device used for the surgical treatment of degenerative disc disease, has been shown to have a stabilizing effect, reducing the stresses contributing to adjacent segment disease, as seen following anterior cervical discectomy and fusion (ACDF). Furthermore, the stringent inclusion criteria for cervical total disc replacement (TDR) limit its use as a surgical option for many patients.

Methods: An electronic literature search across databases in the years up to 2018 was conducted, in English only. The inclusion criteria for studies included all patients over 18 years of age, undergoing single level or twolevel ACDF, CTDR or DCl for cervical degenerative disc disease, refractory to conservative therapy. The primary outcomes of our study were differences in Heterotopic Ossification (HO), ASD and reoperation rates between the groups.

Results: There were 3050 patients analyzed in all studies; 408 patients underwent DCl, 1262 with ACDF, 1380 with CTDR. Mean age ranged from (42.6-53.4) years in the DCI group, (24.6-53.3) in the ACDF group, (40-52) in the CTDR group. The results showed that $\mathrm{DCl}$ is associated with an increased incidence of $\mathrm{HO}$ as compared to CTDR $(p=0.012)$. Additionally, $\mathrm{DCl}$ is associated with a lower reoperation rate as compared to ACDF ( $p=0.015)$. Finally, $D C l$ is associated with lower rates of ASD compared to ACDF, although not significant $(p=0.538)$.

Conclusion: While DCI seems to confer a reduction in reoperation rate, which is a concern with ACDF, further prospective studies are needed to confirm its reliability as an alternative to ACDF and CTDR in the treatment of cervical degenerative disc disease. 
3:49 - 3:51 pm

\section{Does Meyerding Slippage Reduction in Spondylolisthesis Correlate with Patient Outcomes?}

Rory R. Mayer MD; Andrew Kai-Hong Chan MD; Erica F. Bisson MD, MPH, FAANS; Michael S. Virk MD, PhD; Brenton H. Pennicooke MD, MS; Mohammed A. Alvi MD; Eric A. Potts MD; Mohamad Bydon MD; Regis W. Haid; Steven D. Glassman ; Kevin T. Foley MD, FACS, FAANS; Dean Chou MD

Introduction: Reduction of Meyerding grade is often a goal during spondylolisthesis surgery. Although radiographic appearance may improve, correlation with patient reported outcomes (PROs) is rarely reported.

Methods: The Quality Outcomes Database (QOD) was queried for patients undergoing posterior lumbar fusion for spondylolisthesis with minimum 2-year follow up. PROs, including ODI, EQ-5D, numeric rating scale leg and back pain, and satisfaction were noted. Multivariable regression models were fitted for 24-month PROs. Data were analyzed for magnitude of slippage reduction and correlated with PROs. Patients were divided into two groups: $<3 \mathrm{~mm}$ reduction and $\geq 3 \mathrm{~mm}$ reduction.

Results: 206 patients were identified; demographics and clinical characteristics were similarly distributed between the cohorts except for depression, use of interbody, preoperative $\mathrm{mm}$ of spondylolisthesis and percentage of cases with mobile spondylolisthesis which were corrected for in the multivariate analysis. 104 patients underwent lumbar decompression and fusion with slippage reduction $\geq 3 \mathrm{~mm}$ (mean 5.13 , range 3 to 12 ), and 102 underwent surgery with slippage reduction less than $3 \mathrm{~mm}$ (mean $0.44 \mathrm{~mm}$, range -2 to 2 ). Patients in both groups (slippage reduction $\geq 3 \mathrm{~mm}$, and slippage reduction $<3 \mathrm{~mm}$ ) reported significant improvement in all PROs ( $p<0.001$ ). There was no significant difference with regard to the PROs between patients with or without reduction of listhesis on univariate and multivariate analysis.

Conclusion: Significant improvement was found in terms of all patient-reported outcomes in patients undergoingdecompression and fusion for lumbar spondylolisthesis. There was no correlation with clinical outcomes and magnitude of Meyerding slippage reduction. 
3:51 - 3:53 pm

\section{Operative Time Between Conventional Fluoroscopy, Fluoroscopy-based Instrument Navigation, and the Robot in MIS-TLIF}

Timothy Y. Wang MD; Farah Hamouda; Vikram Mehta MPH, MD; Eric W. Sankey MD; Khoi D. Than MD; CR Goodwin; Isaac O. Karikari MD; Muhammad M. Abd-El-Barr MD, PhD

Introduction: Multiple instrument-navigation modalities including CT-guided and robot-assisted methods claim both efficacy and accuracy when applied to spine surgery. However, current navigational systems increase setup and operating times.

Methods: Patients undergoing minimally invasive transforaminal lumbar interbody fusion (MIS-TLIF) using fluoroscopy-based instrument navigation (TrackX) were prospectively enrolled. Age, body mass index (BMI), sex, operative levels, laminectomy, any hardware removal, and total operative time were recorded. These data were then retrospectively compared to previous consecutive MIS-TLIF cases using conventional fluoroscopy as well as robot-assisted (Mazor Robotics) MIS-TLIF.

Results: A total of 120 cases (74 conventional, 14 robot-assisted, 32 Track-X) were included in analysis. Perioperative and demographic data were similar between groups. Average fluoroscopy time for conventional, robot-assisted, and Track-X was $118 \pm 36,157 \pm 41$, and $107 \pm 37$ minutes, respectively, for each MIS-TLIF level ( $p<0.05$ across each group). Time added for decompression or any hardware removal did not significantly differ between groups ( $p>0.05)$. Operative times for robot-assisted cases remained consistently greater than those for conventional fluoroscopy, while those for TrackX trended downwards through approximately 11 patients, at which point operative times were consistently lower than those for conventional MIS-TLIFs. There were no TrackX MIS-TLIF cases that were abandoned in favor of standard fluoroscopy.

Conclusion: TrackX fluoroscopy-assisted instrument navigation during MIS-TLIF, while associated with a short initial learning curve, results in shorter operative times and increased surgeon efficiency when compared to conventional or robot-assisted MIS-TLIF. This is the first navigational system that improves surgeons operative times. 


\section{3:53 - 3:55 pm}

\section{Minimally Invasive TLIF Results in Less Reoperation than Open TLIF}

Barry Cheaney II BS; Diana Ko; Brittany Stedelin; Ahmed M.T. Raslan MBBS MCh; Khoi D. Than MD

Introduction: Transforaminal lumbar interbody fusion (TLIF) is a well-established procedure traditionally performed in an open (O-TLIF) fashion;to minimize tissue trauma, the minimally invasive (MIS-TLIF) approach was developed.

Methods: The authors retrospectively reviewed the medical records of patients who underwent a single-level TLIF procedure by neurological and orthopedic surgeons at a single institution, with at least 6-month follow-up. Patient demographics and surgical details were collected. Data was analyzed by SPSS.

Results: A total of 127 patients met inclusion criteria; 98 O-TLIF (77.2\%) and 29 MIS-TLIF (22.8\%). The groups had similar demographics. There was no difference in mean operative time $(p=0.772)$ or mean hospital stay $(p=0.630)$. MIS-TLIF had less blood loss $(p=0.007)$ and O-TLIF had longer follow-up $(p=0.018)$. Of the 98 O-TLIF procedures, there were $30(30.6 \%)$ reoperations, including $6(20.0 \%)$ due to infection, $10(33.3 \%)$ ASD, $6(20 \%)$ hardware malposition, $3(10.0 \%)$ non-union, and $5(16.7 \%)$ spondylosis. Of the 29 MIS-TLIF procedures, there were $3(10.3 \%)$ reoperations, including 1 (3.4\%) due to infection, $1(3.4 \%)$ ASD, and 1 $(3.4 \%)$ spondylosis. There was no difference in reoperations due to infection $(p=1.000)$, ASD $(p=0.454)$, hardware malposition $(p=0.335)$, non-union $(p=1.000)$, or spondylosis $(p=1.000)$. Collectively, there was a difference between all-cause reoperations $(p=0.031)$ and non-infectious reoperations $(p=0.040)$.

Conclusion: In this study, MIS-TLIF approach resulted in less all-cause reoperations and less reoperations due to adjacent segment disease, hardware malposition, non-union, and spondylosis combined when compared to O-TLIF approach. 
3:57 - 3:59 pm

\section{Risk Factors of 30-days Reoperation after First and Second Interspace Cervical Arthroplasty}

Nida Fatima; John H. Shin MD;

Introduction: The cervical motion conserving procedures to minimize the biomechanical stress on adjacentlevel degenerative disc in the form of Cervical Arthroplasty (CA) has gained wide importance. As such compared to anterior cervical discectomy and fusion (ACDF), it is associated with fewer rate of complications and readmission.

Methods: The patients from American College of Surgeons, National Surgical Quality Improvement Program who underwent CA between 2005 and 2017 were extracted. Multivariable logistic regression analysis (MLR) model was used to evaluate the association between peri-operative clinical characteristics and reoperation following CA. All associations were computed as odds ratio (OR) with 95\% confidence interval $(\mathrm{Cl})$.

Results: Statistical analysis included 986 patients (age: $48.6 \pm 8.4$ years, $57.1 \%$ females) with 7 patients $(0.7 \%)$ who underwent reoperation within 30-days of the cervical disc replacement. The overall complication rate was $0.2 \%$ in our cohort. The patients who were chronic smokers within one year of surgery had 25.8-folds higher likelihood of reoperation within 30-days (OR: $25.8,95 \% \mathrm{Cl}: 4.44-150.6, p<0.001$ ). Furthermore, patients with deep-incisional surgical site infection (SSI) had 4.7-folds were also an independent predictor of 30-day reoperation (OR: 4.7, 95\% Cl: 3.23-47.8, p=0.03). However, none of the other demographic, co-morbidities and laboratory parameter reached statistically significant $(p<0.05)$.

Conclusion: Our results corroborate that smoking status and deep-incisional SSI are predictors of 30-d reoperation in patients with $\mathrm{CA}$. 
3:59 - 4:01 pm

\section{Safety \& Efficacy of Bicortical Pedicle Screw Instrumentation in Lumbar Fusion: Controlled-Cohort Study of 521 Patients}

Robert W. McCabe DO; Doris Tong; Lucas Garmo; Matthew Bahoura BA; Boyd Richards; Teck Mun Soo

Introduction: Pedicle screw instrumentation and fusion is a common treatment for spinal conditions. Bicortical screws placement has been shown to improve biomechanical properties. However, safety and clinical efficacy data on this technique are lacking.

Methods: We retrospectively reviewed the charts of consecutive adults who underwent elective lumbosacral fusion by multiple surgeons in a single institution between 1/2/2014 and 9/30/2015. Bicortical screw instrumentation was defined as a pedicle screw breaching the anterior vertebral body cortex by at least one visible screw thread. A bicortical segment was defined by an interspace with two bicortical screws in each of the upper and lower adjoining vertebra. Radiographs/CT, $>6 \mathrm{~m}$ postoperatively, were evaluated for fusion by an independent neuroradiologist. We evaluated fusion using Suk's XR criteria of crossing trabeculation between vertebral endplates and less than four degrees of motion and Christensen's CT criteria. Using operative reports and hospital adverse outcome registry, we identified accidental intraoperative puncture/laceration, prevertebral hematoma, venous thrombosis and pulmonary embolism. The hospital-reported complications were validated by chart and radiologic review. Univariate statistics and multivariate logistic regression were used. $\mathrm{P}<0.05$ was considered significant.

Results: We evaluated 521 patients for perioperative complications and 757 interspaces for fusion. Six patients did not have a $6-\mathrm{m}$ follow-up and their fusion outcomes were analyzed as non-fusions. Between the bicortical vs. non-bicortical groups, fusion rate was not significantly different $(95.8 \%$ vs. $96.3 \%, p=.737)$ and average Imaging interval was not significantly different $(8.6 \pm 5.1 \mathrm{vs}$. $8.7 \pm 4.7$ months, $p=.67)$. Multivariate logistic regression demonstrated no significant fusion predictor due to the small non-fusion number. No perioperative complication attributable to bicortical screw placement was observed.

Conclusion: Bicortical screw instrumentation provided similar fusion and perioperative complication rate as non-bicortical screw instrumentation in lumbosacral fusion. 
4:01 - 4:03 pm

\section{Comorbidities Do Not Affect Minimum Clinically Important Difference (MCID) for Fusion in Grade 1 Spondylolisthesis}

Joseph Laratta; Leah Carreon; Avery L. Buchholz MD; Andrew Y. Yew MD; Erica F. Bisson MD, MPH, FAANS; Praveen V. Mummaneni MD; Steven D. Glassman

Introduction: Medical comorbidities, particularly anxiety or depression, may potentially influence how patients perceive clinical benefit after surgery. The current study defined and compared MCID thresholds in patients with and without anxiety/depression and obesity undergoing fusion for grade 1 degenerative spondylolisthesis.

Methods: The Quality Outcomes Database (QOD) was queried for patients who underwent lumbar fusion for grade 1 degenerative spondylolisthesis from January 2014 to August 2017. Patient Reported Outcomes (PROs) included Oswestry Disability Index (ODI), EuroQOL5D (EQ-5D), Back (BP, 0-10) and Leg Pain (LP, 010) scores. Both anchor-based and distribution-based methods for MCID calculation were used.

Results: Of 462 eligible cases, 356 (77\%) had complete baseline and 12-month PROs. The MCID values for ODI were similar in patients with and without a diagnosis of obesity (20.58 and 20.69, respectively), and with and without anxiety/depression (24.72 and 22.56, respectively). The MCID values for BP, LP, and EQ-5D were also similar among all groups regardless of method used to determine MCID.

Conclusion: MCID thresholds for ODI, EQ5D, back pain and leg pain in patients with and without anxiety/depression and obesity undergoing spinal fusion for grade 1 degenerative spondylolisthesis are similar. Preoperative clinical and shared decision-making may be improved by understanding that preop medical comorbidities do not affect how patients determine an important clinical change postoperatively. 
Spine Summit Meeting Abstracts, May 2021

4:03 - 4:05 pm

Correlation of Return to Work with Patient Satisfaction After Surgery for Lumbar Spondylolisthesis: A QOD Analysis

Anthony M. DiGiorgio, DO, MHA; Praveen V. Mummaneni MD; Andrew Kai-Hong Chan MD; Paul Park MD; Erica F. Bisson MD, MPH, FAANS; Mohamad Bydon MD; Steven D. Glassman ; Kevin T. Foley MD, FACS, FAANS; Christopher I. Shaffrey MD, FACS; Eric A. Potts MD; Mark E. Shaffrey MD, FAANS, FACS; Domagoj Coric MD; John Knightly; Michael Y. Wang MD, FAANS; Kaiming Fu; Anthony L. Asher MD, FACS; Michael Virk; Panagiotis Kerezoudis; Mohammed A. Alvi MD; Regis W. Haid; Jonathan Slotkin MD

Introduction: Return to work (RTW) is an important outcome measure after surgery for degenerative spine disease.

Methods: QOD was queried for patients undergoing surgery for degenerative lumbar spondylolisthesis. The primary outcome of interest was correlation between RTW and patient satisfaction. Secondarily, satisfied patients were examined to see what patient factors predicted RTW.

Results: There were 276 patients on whom both satisfaction and RTW status were available in the QOD. Of these, $84 \%$ were satisfied with surgery (NASS index 1-2) and 76\% RTW. Of the patients who did not RTW after surgery, $71 \%$ were satisfied with surgery. Of the patients who RTW, $15 \%$ were unsatisfied with surgery (NASS index 3-4). The differences in patient satisfaction between those who did and didn't RTW wasn't statistically significant ( $p>.05$ ). There were significantly more people who had a NASS score of 1 in the RTW group than in the non-RTW group $(72.2 \%$ vs42.6\%,p<.05). Failure to RTW was associated with lower level of education, worse baseline back pain (measured by NRS), and worse baseline disability (measured by ODI).

Conclusion: There are a substantial number of patients who are satisfied with surgery despite not returning to work.Patients who were satisfied with surgery and didn't RTW typically had worse preoperative back pain and ODI and typicallydid not have a college education. While RTW remains an important measure after surgery, physicians should be mindful that patients who do not RTW may still be satisfied with their outcome. 
4:05 - 4:07 pm

\section{Minimally Invasive Posterior Cervical Foraminotomy versus Anterior Cervical Fusion and Arthroplasty: A Systematic Review}

Andrew Platt MD; John E. O'Toole MD, MS

Introduction: Although minimally invasive posterior cervical foraminotomy (MIS-PCF) is frequently employed in the treatment of cervical radiculopathy, there are very few studies directly comparing outcomes between MIS-PCF and anterior cervical discectomy and fusion (ACDF) and between MIS-PCF and cervical total disc arthroplasty (TDA).

Methods: Studies were evaluated for differences in operative/admission outcomes, visual analog scale(VAS), neck disability index(NDI), complications, and reoperation.

Results: Four directly comparative studies including one randomized controlled trial were included in analysis. One study found that average operative time was significantly decreased in the ACDF group. Two studies compared the revision rate after MIS-PCF to ACDF and found that the rate after ACDF ranged from $3.8 \%$ to $4.7 \%$ whereas the revision rate after MIS-PCF ranged from $4.1 \%$ to $6.7 \%$. Neither study found the differences to be statistically significant. One study compared operative/admission outcomes after TDA and MIS-PCF and found average operative time and hospital length of stay to be significantly decreased with MIS-PCF. Two studies compared VAS-A, VAS-N, and NDI after MIS-PCF and TDA and neither study found significant differences between the groups.

Conclusion: Studies comparing MIS-PCF, ACDF, and TDA are limited by short follow-up periods and small sample sizes. The results of this systematic review suggest that MIS-PCF has similar operative/admission outcomes and patient reported outcomes to ACDF/TDA. Further research is necessary to assess which surgical approach is superior in rate of revision. 
4:07 - 4:09 pm

\section{Age as a Predictor for Complications and Outcomes in Multilevel TLIF: Michigan Spine Surgery Improvement Collaborative}

Chad F. Claus DO; Evan J. Lytle DO; Doris Tong; Chenxi Li; Matthew Bahoura BA; Richard Easton MD; Muwaffak Abdulhak MD, FRCS; Peter L. Bono DO; Paul Park MD; Clifford M. Houseman DO; Boyd Richards; Teck Mun Soo

Introduction: The Michigan Spine Surgery Improvement Collaborative (MSSIC) is a quality improvement collaborative with 26 hospitals. Elderly patients undergoing multi-level transforaminal interbody lumbar fusion (MTLIF) are considered high risk for complications. However, data on complications and patient-reportedoutcomes (PRO) are lacking.

Methods: We queried for patients $>50-y-0$ who underwent MTLIF ( $\geq 2$ ) between 3/2014-7/2019 for 90-day complications and PRO (baseline, 90-d, 1-y, 2-y). Complications were defined as major or minor based on Carreon's classification. PRO was measured by VAS back/leg, PROMIS, EQ5D, and NASS patient satisfaction index. Univariate analyses were used to compare the complications cohorts and PRO over time. Multiple Imputation (MI) was used to generate complete covariate datasets. Generalized estimating equation (GEE) was used to identify predictors for complications and PRO using the whole cohort. GEE was repeated for both outcomes in subgroup analyses using the elderly (>70-y-0) cohort. Baseline PRO and complications were used as independent variables in the PRO GEE. Bonferroni's correction was used.

Results: We included 3,120 patients with 961 (31\%) > 70-y-o and 2,159 (69\%) between 50-69. Approximately $30 \%$ of baseline PRO were missing. Age was not predictive in all complication analyses. Number of operative levels was predictive of any $(p=.001)$ and minor $(p=.002)$ complication. Patients had significant PRO improvement $(p=<.001)$ over all intervals. Complication was predictive of worse leg VAS, PROMIS, and satisfaction scores $(p=<.001)$. Smoking was predictive of worse back VAS and satisfaction $(p=<.001)$. Independent preoperative ambulation was predictive of improved PROMIS, and satisfaction $(p=<.001)$. Within the elderly, independent preoperative ambulation and BMI were predictive of improved PROMIS $(p=<.001)$. Complications had no significant effect on PRO in the elderly.

Conclusion: We demonstrated that age was not a predictor of complications or poor PRO. 
4:09- 4:11 pm

\section{Effect Of Cage Type On Subsidence In Transpoas Lateral Lumbar Interbody Fusion}

Ifije Ohiorhenuan MD, PhD; Corey T. Walker MD; Jakub Godzik; Jay D. Turner MD, PhD; Juan Uribe

Introduction: Subsidenceof an implant can lead to the failure of a lateral lumbarinterbody fusion (LLIF) to indirectly decompress neural elements.

Methods: A retrospective, single-center study of 94 consecutive patients whounderwent LLIF without direct decompression was performed.Radiographic measurements were taken from pre-operative andpost-operative images, including immediate post-operative computedtomography. Radiographicsubsidencewas measured using a four-pointgrading scheme (Malham et al., 2015). Clinical subsidence was determined by a chart review of post-operative course.

Results: Radiographic subsidence was observed in 18 of 185 treated levels (12\%). In 10 cases, subsidence occurred intra-procedurally, with intra-operative imaging showing subsidence occurring during graft placement or endplate preparation with the cobb or box cutter instruments. In 7 cases, new subsidence was found at 6-week follow-up and in 1 case at 12-week follow-up. No subsidence was seen after 12 weeks and average follow-up across groups was 31.3 weeks. Type-2 subsidence (end plate violation on both lateral sides of the inferior end plate) was seen in 15 cases. Type-3 subsidence (superior and inferior end plate violations) was observed in 3 cases. Subsidence rates were as follows: L1/2, 17\% (2/12); L2/3 13\% (4/30); L3/4 12\% (7/58) and L4/5 8\% (5/66). Subsidence in PEEK implants was seen in $18 / 154$ levels (12\%) but none was observed in 31 levels treated with Titanium implants. This was statistically significant ( $p<0.05$, Chi-squared test). Clinical subsidence was observed in 3 of 18 patients with radiographic subsidence $(17 \%)$. This was managed conservatively, and no patient underwent posterior decompression.

Conclusion: Radiographicsubsidenceoccurred at a rate of $13.6 \%$ of LLIFs andclinicalsubsidenceoccurred at a rate of 3\%.Subsidencemost oftenresulted from an intra-operative endplate violation and was seen morefrequently in PEEK implants than titanium. 
4:11- $4: 13$ pm

\section{Cost Comparison of Single Level Posterior and Anterior/Lateral Lumber Interbody Fusions}

Shashank V. Gandhi; Justin Noble Virojanapa; Ahmad Latefi DO

Introduction: Some patients with single-level lumbar degenerative disc disease (LDDD) may be candidates for either posterior, anterior, or lateral lumbar interbody fusions.

Methods: All patients from 2017-2019 at this institution who underwent PLIF/TLIF or ALIF/LLIF were assessed. Patient specific hospital metrics including post-anesthesia care unit (PACU) length of stay (LOS), intensive care LOS, hospital LOS, foley-catheter days, discharge disposition, use of assistive devices, 30-dayreadmission rates, operating room cost, and hospital costs were assessed for each group.

Results: 314 patients were assessed: 220 in the PLIF/TLIF group and 94 in ALIF/LLIF group. There was no difference in gender between the groups. The patients in the ALIF/LLIF group were younger (means 54.9 versus 60.2 years, $p=0.012)$. The PLIF/TLIF group had significantly shorter mean foley-catheter days $(2.3$ versus 2.9 days, $p=0.030$ ). The PLIF/TLIF group compared to ALIF/LLIF had no significant difference in PACU LOS ( 0.35 versus 0.32 days, $p=0.389$ ), ICU LOS ( 3.7 versus 1.3 days, $p=0.137$ ), or hospital LOS (6.2 versus 6.8 days, $p=0.406)$. There was similar postoperative use of assistive devices for ambulation $(72.3 \%$ PLIF/TLIF versus $75.7 \%$ ALIF/LLIF, $p=0.533)$. There was no difference in discharge to rehabilitation facility rates $(24.5 \%$ PLIF/TLIF versus $20.2 \%$ ALIF/LLIF, $p=0.405)$. 30-day-readmission rates were similar (10.9\% PLIF/TLIF versus 9.6\% ALIF/LLIF, $p=0.724)$. However, PLIF/TLIF group had significantly lower operating room cost $(\$ 18,504$ versus $\$ 23,775 ; p<0.001)$ and hospital cost $(\$ 74,350$ versus $\$ 90,629 ; p=0.001)$.

Conclusion: In the immediate 30-day postoperative period, PLIF/TLIF is more cost-effective and has better value than ALIF/LLIF with lower operating room and hospital costs and fewer foley-catheter days resulting in similar length of stay metrics, discharge to rehabilitation facility, and 30-day-readmission rates for single-level LDDD. Long-term data is needed to quantify outcomes and complications such as pseudoarthrosis and adjacent segment disease. 
4:13-4:15 pm

\section{A Cost Benefit Analysis of Increasing Surgical Technology in Lumbar Spine Fusion}

Peter G. Passias MD; Avery Brown; Haddy Alas; Cole Bortz BA; Katherine Pierce; Waleed Ahmad; Sara Naessig; Erik Wang; Hamid Hassanzadeh; Lawal Labaran; Varun Puvanesarajah BS; Aaron J. Buckland MBBS, FRACS; Michael Gerling

Introduction: Numerous advances have been made in spinal fusion, such as minimally invasive (MIS) robotic-assisted surgery. However, it is unknown how these advances impact cost of care.

Methods: Patients $\geq 18$ yo undergoing lumbar fusion surgery included. Patients categorized into 3 groups based on procedure type: open, MIS, robotic. Open included posterior spinal fusion. MIS included TLIF or LLIF with percutaneous screws. Robotic included robot-assisted interbody fusion. Propensity score matching (PSM) among groups for number of levels fused. Costs calculated using PearlDiver database. For robotic cases, costs reflective of operational fees \& initial purchase costs. Complications \& comorbidities (CC) \& major CC (MCC) assessed according to CMS.gov manual definitions. QALY \& cost/QALY calculated using 3\% discount rate to account for residual decline to life expectancy (78.7 years). Cost/QALY calculated for Y1 \& life expectancy, assuming no loss of benefit.

Results: 360 PSM patients (120 open, 120 MIS, 120 robotic) included. Descriptive stats: $58.8 \pm 13.5 y r s, 50 \% F$, BMI29.4 \pm 6.3 , op time 294.4 $\pm 19 \mathrm{~min}$, LOS 4.56 \pm 3.31 days, EBL 515.9 $\pm 670 \mathrm{cc}, 2.3 \pm 2.2$ levels fused. Postop complication rate highest in robotic $(p<0.05)$. Revision rates comparable among groups $(p>0.05)$. Factoring in complications, revisions, purchasing \& operating fees, costs of robotic cases highest (Fig 1,p<0.05). Subanalysis of 42 patients with BL \& Y1 EQ5D data: Y1 cost/QALY for open, MIS, robotic was \$296,624.48, $\$ 115,911.69, \$ 592,734.30$, respectively. If utility gained sustained to life expectancy, cost/QALY was, respectively, $\$ 14,905.75, \$ 5,824.71, \$ 29,785.64$.

Conclusion: Matching for levels fused, robot-assisted patients had $30 \%$ higher costs of surgery rate of complications than MIS open spine surgery patients. While Y1 economic outcomes werent optimal for robotic surgery cases, projected costs per QALY at life expectancy well below established acceptable thresholds. 
4:15 - 4:17 pm

Two Year Comparative Effectiveness of Adjustable Lordotic Expandable vs. Static Lateral Lumbar Interbody Fusion Devices

Yan M. Li MD, PhD; Richard F. Frisch MD; Zheng Huang; James Towner MD; Yanicy Li PhD; Jessica

Riggleman; Charles Ledonio MD, CCRP

Introduction: Static interbody spacers may cause iatrogenic endplate damage and implant subsidence due to forceful impaction and excessive trialing. Expandable interbody spacers with adjustable lordosis offer in situ expansion that may optimize endplate contact and maximize and maintain sagittal correction until fusion occurs.

Methods: Multi-surgeon, retrospective, IRB-exempt chart review of consecutive patients who underwent MIS LLIF at 12 contiguous level(s) using either a polyetheretherketone (PEEK) static (27 patients) or a titanium expandable spacer with adjustable lordosis (24 patients).

Results: For VAS back pain scores, mean improvement from preoperative to 6, 12, and 24 months was significantly greater in the expandable group compared to the static group by $51.7 \%, 33.6 \%$, and $73.8 \%$, respectively $(\mathrm{P}<0.05)$. For ODI scores comparing both groups, mean improvement from preoperative to 3,6 , 12 , and 24 months was significantly greater in the expandable group by $56.2 \%, 73.5 \%, 75.5 \%$, and $109.2 \%$, respectively, compared to the static group $(\mathrm{P}<0.05)$. The mean improvement in neuroforaminal height from preoperative to 6 weeks, 3 months, and 6 months was significantly greater in the expandable group compared to the static group $(\mathrm{P}<0.05)$. The mean improvement in segmental lordosis from preoperative to 6 weeks, 3,6 , and 12 months was significantly greater in the expandable group compared to the static group $(\mathrm{P}<0.05)$.

Conclusion: This study showed significant positive clinical outcomes for patients who underwent MIS LLIF using titanium expandable interbody spacers with adjustable lordosis. 


\title{
4:17 - 4:19 pm
}

\section{Comparing the Surgical Outcomes of Dynamic Cervical Implants vs Total Disc Replacement and ACDF}

\author{
Mohamad Bydon MD; Yagiz U. Yolcu MD; Waseem Wahood; Atiq Bhatti; Aya Akhras; Anshit Goyal MBBS
}

Introduction: Dynamic Cervical Stabilization, first introduced by Matge in 2004, has been introduced in Europe as a new device for the surgical treatment of degenerative disc disease. The Dynamic Cervical Implant (DCl; Paradigm Spine) has been shown to have a stabilizing effect, thus reducing the stresses contributing to ASD, as seen following ACDF. Furthermore, the stringent inclusion criteria for CTDR limit its use as a surgical option for many patients.

Methods: A literature search across numerous databases in the years up to 2018 was conducted, in English only. The inclusion criteria for studies included all patients over 18 years of age, undergoing either 1-2 level ACDF, CTDR or DCl for cervical degenerative disc disease, refractory to conservative therapy. The primary outcomes of our study were differences in Heterotopic Ossification (HO), Adjacent Segment Disease (ASD) and reoperation rates between the groups.

Results: There were 3050 patients analyzed in all studies; 408 patients underwent DCI, 1262 with ACDF, 1380 with CTDR. Mean age ranged from (42.6-53.4) years in the DCI group, (24.6-53.3) in the ACDF group, (40-52) in the CTDR group. The results showed that $\mathrm{DCl}$ is associated with an increased incidence of $\mathrm{HO}$ as compared to CTDR $(p=0.012)$. Additionally, $\mathrm{DCl}$ is associated with a lower reoperation rate as compared to ACDF ( $p=0.015)$. Finally, $D C l$ is associated with lower rates of ASD compared to ACDF, although not significant $(p=0.538)$.

Conclusion: While $\mathrm{DCl}$ seems to confer a reduction in reoperation rate, a great concern with ACDF, further prospective trials will need to confirm its reliability as an alternative to ACDF and CTDR in the treatment of cervical degenerative disc disease. 


\section{4:19 - 4:21 pm}

\section{Percutaneous Lumbar Interbody Fusion: Short Term Radiographical and Quality of Life Outcomes}

Vikram Mehta MPH, MD; Timothy Y. Wang MD; Eric W. Sankey MD; Mostafa A. Gabr MD, MHSc; Alexia Bwensa; CR Goodwin ; Isaac O. Karikari MD; Muhammad M. Abd-El-Barr MD, PhD

Introduction: Minimally-invasive transforaminal lumbar interbody fusion is a common procedure in lumbar spine fusion. Typically, facetectomy is necessary to access the disc space for cage placement. Most authors have described the use of endoscopy to insert static cages through this approach or the use of a porous allograft-containment mesh. In this study, we describe percutaneous access to the disc and insertion of an expandable titanium cage through Kambins triangle without facetectomy.

Methods: A retrospective review of patients undergoing single-level percLIF without the use of an endoscope for grade 1 lumbar spondylolisthesis via Kambins triangle with an expandable cage was performed.

Demographic information, Oswestry disability index, pre- and postoperative radiographic factors, perioperative data, and complications were recorded from the electronic medical record.

Results: Eleven total patients (two males) were included in this study. Anterior disc height significantly improved at six weeks, however, lost significance at six months. Posterior disc height significantly improved at six weeks and six months. Oswestry disability index was significantly improved by $24.2 \%$ from baseline to 12 months postoperatively $(p<0.05)$.

Conclusion: Initial experiences have shown than percLIF without the use of an endoscope with an expandable titanium cage through Kambins triangle is a safe and clinically efficacious procedure for reducing grade 1 lumbar spondylolisthesis and radiculopathy. 


\section{4:21 - 4:23 pm}

\section{Patient Reported Outcomes Following Lumbar Disc Herniation: Comparison of a Universal and Multitier Health Care System}

Oliver GS Ayling MD, MSc; Tamir T. Ailon MD, MPH; Nicolas Dea MD, MSc, FRCSC; Raphaele CharestMorin; Charles Fisher MD

Introduction: Canada has a government funded health systemandaccess to spinal surgeons requires a referral. The United States utilizes a combined public and private payer system where patients may directly access specialists.

Methods: Surgical lumbar disc herniation patients treated in Canada enrolled in the Canadian Spine Outcome Research Network (CSORN) prospective registry were compared with the surgical cohort enrolled in the Spine Patients Outcome Research Trial (SPORT) study.

Results: There were 443 and 573 patients in the CSORN and SPORT cohorts. Patients in CSORN were older (46.2 \pm 13.2 vs. $41.6 \pm 10.9, p<0.001)$, had higher rates of smoking $(32.0 \%$ vs. $22.8 \%, p<0.001)$, and higher employment rates $(66.9 \%$ vs. $61.3 \%, p=0.034)$. The CSORN cohort had a lower Owestry Disability score at baseline $(50.5 \pm 15.1$ vs. $55.7 \pm 19.6, p<0.01)$ but a higher proportion of patients with symptom durations greater than 6 months $(44.5 \%$ vs. $21.1 \%, p<0.0001)$. The CSORN cohort demonstrated significantly greater rates of satisfaction after surgery at 3 months $(74.8 \%$ vs. $65.3 \%, p=0.003)$ and 1 year $(81.4 \%$ vs. $68.7 \%, p<0.001)$. CSORN was a significant predictor of patient satisfaction at 1 year on multivariable regression $(\mathrm{OR} 1.3,95 \% \mathrm{Cl}$ $1.29-1.49, p<0.001)$.

Conclusion: Patients undergoing surgery for lumbar disc herniation in Canada (CSORN cohort) reported higher rates of satisfaction at 3 months and 1 year post-operatively compared to the United States cohort (SPORT) despite having longer durations of symptoms prior to surgery. 


\section{$4: 23-4: 25$ pm}

\section{One-Level Versus Two-Level Cervical Disc Arthroplasty: A Biomechanical Study}

Hoon Choi MD, MS; Yuvaraj Purushothaman MS; Jamie Baisden MD; Davidson Jebasselan; Narayan Yoganandan

Introduction: Cervical disc arthroplasty (CDA) is a motion preserving alternative to anterior cervical discectomy and fusion (ACDF). Following FDA approval for two-level CDA, patients and surgeons are opting for two-level CDA instead of two-level ACDF.

Methods: A previously validated C2-T1 osteoligamentous finite element model (FEM) was used. FEM simulated cortical bone, cancellous bone, annulus ground substance, annulus fibrosus, nucleus pulposus, anterior longitudinal ligament (ALL), posterior longitudinal ligament (PLL), facet joints with capsular ligament, ligamentum flavum, and interspinous ligament. A commercially available CDA device FDA-approved for two consecutive levels (Mobi-C; Biomet Zimmer LDR) was modeled. CDA was simulated at C5-6 and C6-7 levels.

Results: CDA increases range of motion (ROM) and facet force (FF) at the index level and decrease intradiscal pressure (IDP) at the adjacent levels. Two-level CDA leads to less extreme elevations in ROM and FF at the index levels. IDP declines were comparable in one and two-level CDA models.

Conclusion: CDA changes biomechanics in all three columns of the spine at index and adjacent levels. Twolevel CDA leads to less extreme biomechanical changes at the index levels by distributing the forces over two levels. 
Sunday, March 8, 2020

General Spine Surgery 1/Trauma/Spinal Cord Injury

Abstract Breakout Session

\section{7:30 - 7:32 am}

\section{Predictors of Thirty-day Complications Following Anterior Cervical Fusion}

Nida Fatima; John H. Shin MD

Introduction: Rate of medical and surgical complications following anterior cervical fusion (ACF) vary widely depending upon the clinical-, diagnosis-, and procedure-related factors.

Methods: A total of 3,316 patients from the American College of Surgeons, National Surgical Quality Improvement Program who underwent ACF between 2005 and 2017 were extracted.

Results: The 30 -d complications for the 3,316 patients (62.2\% females, mean age: $57.8 \pm 13.8$ years) following ACF was $90(3.4 \%)$. The most common complications were bleeding disorders $(n=28,0.8 \%)$, reintubation $(n=25,0.7 \%)$, pneumonia $(n=21,0.6 \%)$, and urinary tract infection $(n=17,0.5 \%)$. Univariate analysis revealed that age $>55$ years $(p<0.001)$, female gender $(p=0.02)$, history of chronic obstructive pulmonary disease $(p=0.007)$, dialysis $(p=0.04)$, disseminated cancer $(p=0.04)$, long-term steroid use $(p=0.008)$, functional status $(p=0.03)$, blood transfusions $(p=0.04)$, American Society of Anesthesiologists (ASA) II $(p<0.001)$, ASA III $(p<0.001)$, preoperative serum White Blood Cell Count $(p=0.04)$, total operative time $(p<0.001)$ and duration of anesthesia $(p<0.001)$ were significantly associated with complications. However, the MLR model indicated that (i) female gender (OR: $1.79,95 \% \mathrm{Cl}: 1.00-3.21, \mathrm{p}=0.04$ ), (ii) long-term pre-operative steroid use (OR: 2.84 , 955Cl: 1.02-7.81, $p=0.04$ ), and (iii) total operative time (OR: 1.00, 95\% Cl: 1.00-1.01, $p<0.001$ ) were significant independent predictors of 30-d complications.

Conclusion: This is the largest study to date of the patients undergoing ACF and provides a set of predictive variables that accounts for patient and surgical-related factors which can contribute to patient-counselling and accurate risk-adjustments. 


\section{7:32 - 7:34 am}

\section{Association between Intraoperative ALIF Complications and Number of Prior Abdominal Surgeries}

Arbaz Momin; Edward Barksdale; Zaeem Lone; Jacob Enders BSE; Michael P. Steinmetz MD

Introduction: Anterior lumbar interbody fusion (ALIF) requires mobilization of intra-abdominal viscera/vasculature to access the anterior spine. The approach to the spine can become complicated as scarring and/or adhesions develop from prior abdominal surgeries, increasing risk of intraoperative complications.

Methods: Records of 660 patients who underwent ALIF between 2008 and 2018 were retrospectively reviewed. Patient demographics, Charleston Comorbidity Index $(\mathrm{CCl})$, level of fusion, past abdominal surgical history, use of access surgeon during exposure, intraoperative, and postoperative complications were collected. Predictors of intraoperative and postoperative complications were analyzed using simple and multivariable logistic regression.

Results: The odds of developing an intraoperative complication increases 36\% for each additional prior abdominal surgery (OR 1.36, [95\% Cl: 1.09-1.68]). After controlling for age, BMI, preoperative comorbidities $(\mathrm{CCl})$, multilevel exposure, and the use of vascular access surgeon the odds of developing an intraoperative complication remained statistically significant (OR1.38, [95\% Cl 1.10-1.74]). Number of prior abdominal surgeries was not a significant predictor of postoperative complications.

Conclusion: With each additional prior abdominal surgery, accumulation of scarring and adhesions can likely obscure anatomical landmarks and increase the odds of developing an intraoperative complication. Therefore, history and quantity of prior abdominal surgeries should be taken into consideration during planning and operative exposure of the anterior spine via a retroperitoneal approach. 


\section{7:34 - 7:36 am}

\section{Chiropractic Adjustment and Temporal Association with Vertebral Artery Dissections}

Akash Mitra; Hooman Azad; Nikil Prasad BS; Michael Cloney MD, MPH; Nader S. Dahdaleh MD

Introduction: Vertebral artery dissections (VADs) are rare yet potentially devastating events, and chiropractic vertebral artery dissections (cVADs) are a known subset of VADs that remain understudied. This study presents a large cohort of these patients and analyzes predictors of outcomes among them.

Methods: 310 patients who were treated at a level 1 trauma center for VADs from 2004-2018 were included in this study. Patient, event, and treatment characteristics were collected, including: demographics, Charlson Comorbidity Index, cause of VAD (traumatic vs. spontaneous), location of VAD, complications, medical therapy (anticoagulation vs. antiplatelet), and modified Rankin scores (mRS) at various times.

Results: Of our 310 patient cohort, $34(11 \%)$ had recent chiropractic manipulation prior to onset of their VAD. As compared to the general cohort, we found cVADs occurred more frequently in those were younger $(p=0.01)$, healthier $(p=0.05)$. cVADs were more frequently bilateral $(p=0.048)$, had fewer segments of the vertebral artery affect $(p=0.02)$, had fewer $30 d$ readmissions $(p=0.07)$, and had similar clinical resolution $(p=0.98)$.

Conclusion: In this study we found that cVADs preferentially occurred in a younger and healthier demographic. cVADs also tended to be more bilateral, affect a smaller region of the vertebral artery. However, despite the unique nature of the inciting event these individuals demonstrated a similar propensity for clinical resolution, and had lower $30 \mathrm{~d}$ readmission rates. 
7:36:00 AM - 7:38:00 AM

\section{The Effect of Anticoagulation vs. Antiplatelet Therapy on Neurologic Status Following Vertebral Artery Dissections}

Nikil Prasad BS; Hooman Azad; Akash Mitra; Michael Cloney MD, MPH; Nader S. Dahdaleh MD

Introduction: Vertebral artery dissections (VADs) are rare yet potentially devastating events. Due to their rarity, there is a paucity of quantitative literature analyzing the predictors of positive outcomes. Typically, these events are managed medically, with the end goal of preventing clot formation to reduce stroke risk. The two principal methods of medical management for VADs are anticoagulation and antiplatelet therapy.

Methods: Charts for 310 patients who were treated at a level 1 trauma center for VADs from 2004-2018 were included in this study. Patient, event, and treatment characteristics were collected, including: demographics, Charlson Comorbidity Index, cause of VAD, location of VAD, complications, medical therapy (anticoagulation vs. antiplatelet), and modified Rankin scores (mRS) at various times.

Results: There were improved clinical outcomes among those patients on anticoagulation, with an average change in $\mathrm{mRS}$ of $-0.82 v$. $-0.64(p=0.02)$ for antiplatelet therapy, despite similar mRS at VAD onset (Tables $2,3)$. Anticoagulation was used more frequently in those with radiographic signs of occlusion $(p=0.03)$ and those who were younger $(p=0.01)$, healthier $(p=0.02)$, and female $(p=0.04)$. In a multivariate model, only initial mRS was significantly associated with clinical improvement $(p<0.001)$.

Conclusion: Anticoagulation is not associated with improved clinical outcomes from VADs after controlling for other confounders. Therefore, the most important consideration in selection of medical management is the presence of other comorbid conditions requiring anticoagulation. 
7:38 - 7:40 am

\section{Utilization of Intraoperative Neuromonitoring During the Treatment of Cervical Myelopathy}

Michael Karsy MD, PhD, MSc; Austin Gamblin BS; Al-Wala Awad MD, BS; Jian Guan MD; Marcus D. Mazur MD; Erica F. Bisson MD, MPH, FAANS; Orhan Bican; Andrew T. Dailey MD

Introduction: Cervical myelopathy represents an increasingly more prevalent spinal pathology with significant morbidity. The use of intraoperative neuromonitoring (IONM) has been a common practice in an attempt to detect nervous system injuries when they are reversible and thus mitigate new postoperative neurologic deficits.

Methods: A retrospective review of patients who underwent surgical treatment of cervical myelopathy was performed to evaluate patients who underwent IONM changes from 1/2018 to 8/2018.

Results: Among 366 screened patients, a total of 42 patients (58.2 \pm 16.3 years, $54.8 \%$ males) showed IONM changes compared to 89 patients without IONM change. The most common Nurick grade was $1(n=21,50.0 \%)$ with patients undergoing anterior $(61.9 \%)$, posterior $(33.3 \%)$ or combination $(4.8 \%)$ surgical approaches. The most common instrumented level was C4 (71.4\%) followed by C5 $(66.7 \%)$ with an average of $3.7 \pm 1.9$ levels and corpectomy used in $7(16.7 \%)$ cases. Among the 42 patients with IONM changes, 3 patients showed postoperative deficits ranging from worsened sensory issues, bowel incontinence, and specific or multiple radicular motor deficits all preceded by IONM changes. All 3 patients showed baseline deficits prior to surgical treatment. Reasons for IONM changes varied: electromyography ( $n=25,59.5 \%)$, somatosensory evoked potentials $(n=14,33.3 \%)$, motor evoked potentials $(n=13,31.0 \%)$. Among the 89 patients without an IONM change, 4 showed worsened postoperative deficits, which were also seen at last followup. A sensitivity of $42.86 \%$ and specificity of $68.55 \%$ for predicting post-operative neurological change by IONM was seen. The majority of patients $(124 / 131,94.7 \%)$ with IONM showed no worsened neurological deficit.

Conclusion: Neurological deficits after treatment of cervical myelopathy remain challenging to predict and prevent. Use of multimodal IONM as well as multidisciplinary collaboration among providers and bundled protocols may be a strategy to achieve best outcomes. 
7:40 - 7:42 am

How Does Bias Influence SCI Care? A National Study of Gender and Age in Surgical Decision Making

Theresa Williamson MD; Sarah E. Hodges BA; Zidanyue Yang; Isaac O. Karikari MD; C. Rory Goodwin MD, PhD; Shivanand P. Lad MD, PhD; Muhammad Abd-El-Barr

Introduction: $\mathrm{SCl}$ is a large public health problem with management controversy. Disparities have been identified in disposition to rehab in SCI (Lad 2013). We performed a study to evaluate gender and age differences in surgical intervention and interhospital transfer, possible mechanisms for outcome disparities in SCl.

Methods: Retrospective analysis of patients with spinal cord injury from the National Trauma Database (20112014). The primary objective of the study was to determine the effect of surgical timing, hospital center type, and transfer on discharge outcomes. This study is the result of a secondary analysis to determine the interaction of age, gender and surgical timing and transfer. We modeled the probability of surgical intervention (Yes/No) as a function of trauma center levels, adjusted for teaching status, inter-hospital transfer, region, age, sex, race, ethnicity, payment method, and multiple medical markers. Logistic regression analysis was used for the multivariable analysis.

Results: 21,721 patients had SCI in the cohort and 11,744 met criteria for the study. 61.7 percent of patients received surgery. $75 \%$ of patients were male. Median age was 45 . Elder patients (OR 0.792, Cl 0.769-0.815, $\mathrm{p}<0.001)$ and females $(0.900, \mathrm{Cl} 0.816-0.993, \mathrm{p}=0.0363)$ were less likely to receive surgery. Elder patients were less likely to receive early surgery within 24 hours (OR 0.846, Cl 0.81700.875, p < 0.0001). Among transferred and non-transferred patients, elder patients are less likely to receive surgery.

Conclusion: In this study, women and elderly patients were less likely to receive surgery. If they received surgery, elderly patients were less likely to receive surgery early. Transfer status did not influence this result. Further study is warranted to address mechanisms of gender and age bias in $\mathrm{SCl}$ care. 
7:42 - 7:44 am

\section{Correlation Between Neurosurgery and ISNCSCI Motor Scores After Acute Spinal Cord Injury: A TRACK-SCI Study}

John F. Burke MD, PhD; Anthony M. DiGiorgio DO, MHA; Leigh H. Thomas BA; Xuan Duong Fernandez BA; Lisa U. Pascual MD; Jonathan Pan; Debra P. Hemmerle RN, MSN; Vineeta Singh; Abel Torres; Ferguson Adam PhD; Jacqueline Bresnahan; Michael Beattie; Sanjay S. Dhall MD

Introduction: The International Standards for Neurological Classification of Spinal Cord Injury (ISNCSCI) assessment is considered the gold standard for evaluation of neurologic function after spinal cord injury (SCI). However, the length of the ISNCSCI examination occasionally makes it impractical, with some trials having little over a third of eligible ISNCSCI examinations completed.

Methods: We prospectively collected data on all patients with $\mathrm{SCl}$ as a part of the Transforming Research and Clinical Knowledge in Spinal Cord Injury (TRACK-SCI), which is a prospective, multi-center research collaboration. Patients are evaluated using full ISNCSCI exams while admitted. Separately, neurosurgery motor exams are done daily while in the hospital. We found all instances where ISNCSCI and neurosurgery exams were performed on the same day, and correlated the motor scores across each exam.

Results: We collected data on 113 patients with $\mathrm{SCI}$, including 60 separate examinations with full ISNCSCI and neurosurgery motor scores. We found that the overall motor scores were highly correlated (correlation coefficient $=0.99, p<1 e-50)$. The upper extremity examinations were slightly more variable, but still significantly correlated (correlation coefficient $=0.98, p<1 e-40$ ). We then used the neurosurgery motor score to estimate the AIS grade, and found that the neurosurgery motor score was able to predict AIS grade correctly in $75.76 \%$ of cases. Furthermore, when AIS grades of identical motor scores were grouped together $(A+B, D+E)$, neurosugery motor score AIS prediction accuracy increased to $97.0 \%$.

Conclusion: Neurosurgery motor examinations show good correlation with the motor score of the ISNCSCI examination. Given the time and training that go into regular collection of ISNCSCI examinations, allowingthe neurosurgery motor exam to act as a surrogate for the full ISNCSCI examinationwill only expand the data available for spinal cord research. 
7:44 - 7:46 am

\section{Operative versus Non-Operative Treatment of Geriatric Odontoid Fractures: A Study of North American Trauma Centers}

Jetan H. Badhiwala MD; Christopher D. Witiw MD; Jefferson R. Wilson MD, PhD; Leo da Costa MD; Avery Nathens; Michael G. Fehlings MD, PhD, FRCS(C), FACS

Introduction: With the aging population, the optimal management of geriatric type 2 odontoid fractures has become an increasingly relevant clinical problem.

Methods: Data were derived from the American College of Surgeons (ACS) Trauma Quality Improvement Program (TQIP) database for 2016. Patients $\geq 65$ years old with an acute type 2 odontoid fracture were identified by ICD-10-CM codes. Cases of posterior C1-2 fusion were identified by ICD-10-PCS codes. Propensity score matching of non-operative versus operative treatment was performed in 1:1 ratio adjusting for age, sex, race, comorbidities, mechanism of injury, and ISS. The primary outcome was inpatient mortality. Secondary outcomes included inpatient morbidity (MI, cardiac arrest, DVT, PE, AKI, stroke, sepsis, VAP), hospital length of stay (LOS), and discharge destination. Kaplan-Meier survival curves were created for inpatient mortality by treatment group, and these were statistically compared by the log-rank test.

Results: A final cohort had 506 elderly patients with an acute type 2 odontoid fracture (mean age, 77.8 yrs). Baseline characteristics were balanced between matched non-operative $(N=253)$ and operative $(N=253)$ treatment groups. Inpatient mortality was significantly greater in the non-operative treatment group $(8.7 \%$ vs. $2.4 \%, \mathrm{P}=0.004)$. Kaplan-Meier analysis reveled significantly shorter survival among patients treated nonoperatively $(P<0.001)$. There were no differences in inpatient complications between treatment groups. Patients treated operatively had a longer inpatient hospital stay on average (11.6 vs. 6.3 days, $\mathrm{P}<0.001)$. Most patients who underwent posterior C1-2 fusion were discharged to either a rehabilitation (28.1\%) or skilled nursing $(41.1 \%)$ facility.

Conclusion: These data support a short-term survival advantage among elderly patients with an acute type 2 odontoid fracture who are treated operatively with posterior C1-2 fusion, over those managed non-operatively. 
7:46 - 7:48 am

\title{
Candidates for Percutaneous Pedicle Screw Fixation without Fusion in Thoracolumbar Fractures
}

\author{
Hazem AlKosha; Ahmed Albayar; Sherif A. Omar; Ali Kemal Ozturk MD; Basem I. Awad MD
}

Introduction: Despite the recent immense growth in popularity of percutaneous screw fixation (PFS), the best candidates for PSF without fusion have not been identified in literature. While several classifications for thoracolumbar spinal injuries have been described, Thoracolumbar Injury Classification System (TLICS) has proven acceptable reliability and validity owing to its inclusion of both; neurological status and the morphological features of the injuries.

Methods: A retrospective cohort of patients who underwent PSF were split into 3 TLICS-score categories, then matched with groups having similar scores managed either non-operatively or via open screw fixation (OSF). Each category was assessed for corrective power and loss of correction by comparing initial and 1-year cobb angles as well as Oswestry Disability Index (ODI) and rates of fracture healing at 1 year.

Results: 102 patients (40 females) with age range 19 to 51 years, were admitted 1-25 hours following trauma. Each of TLICS categories consisted of matched treatment groups for comparison. In TLICS-3 fractures (2 treatment groups, $n=12$ each), PSF showed similar outcomes but longer time to ambulation and length of stay (LOS) compared to non-operative management. In TLICS-4 fractures (3 treatment groups, $n=18$ each), PSF showed comparable corrective power and outcomes as OSF but was better in terms of operative time, blood loss, time to ambulation, LOS and cosmesis. Despite higher LOS when compared to non-operative cases, PSF showed superior radiologic and functional outcomes. In TLICS-5 fractures (2 treatment groups, $n=12$ each), PSF showed shorter admissions and time to ambulation but lower corrective power, functional recovery and tendency to lower healing rates.

Conclusion: Isolated PSF is a valid choice in managing TLICS-4 thoracolumbar fractures, however, it did not surpass conventional methods in TLICS-3 or TLICS- 5 fracture types. Further studies are needed before the generalization of findings. 
7:48 - 7:50 am

\section{First in Human Implementation of a Novel Spinal Cord Perfusion Pressure Protocol in Acute Traumatic Spinal Cord Injury}

John K. Yue MD; Debra P. Hemmerle RN, MSN; Ethan Winkler; Leigh H. Thomas BA; Xuan Duong Fernandez BA; Nikolaos Kyritsis PhD; Jonathan Pan; Lisa U. Pascual MD; Vineeta Singh; Philip R. Weinstein MD; Jason Talbott; J. Russell Huie PhD; Ferguson Adam PhD; William Whetstone; Geoffrey T. Manley MD, PhD; Michael Beattie; Jacqueline Bresnahan; Praveen V. Mummaneni MD; Sanjay S. Dhall MD

Introduction: Mean arterial pressure (MAP) targets cannot fully account for changes in intraspinal pressure for maintaining spinal cord perfusion pressure (SCPP) during acute traumatic spinal cord injury (SCI) management.

Methods: Starting 12/2017, blunt SCI patients presenting $<24$ h of injury with admission American Spinal Injury Association Impairment Scale (AIS) A-C (or AIS D at neurosurgeon discretion) received lumbar subarachnoid drain (LSAD) placement for SCPP monitoring in the intensive care unit. This is known as the SCPP Protocol, and comprises standard care at our institution. SCPPs were monitored for 5 days with goal $\geq 65 \mathrm{mmHg}$ achieved through intravenous fluids and vasopressor support. AIS grades were assessed at admission and day 7 .

Results: In 15 patients enrolled to date, age was $60.5 \pm 17.0$-years. Injury level was $93.3 \%$ cervical, $6.7 \%$ thoracic. Admission AIS were 20.0\%/20.0\%/26.7\%/33.3\% for A/B/C/D. Fourteen patients underwent surgical decompression with time-to-surgery $8.8 \pm 7.1 \mathrm{~h}(71.4 \%<12 \mathrm{~h})$. Length of stay was $14.7 \pm 8.3$-days. None had LSAD-related complications. Seven patients had respiratory complications and one expired after family transitioned to comfort care. Thirty-three percent experienced a one-grade AIS improvement.

Conclusion: In our initial experience of 15 patients with acute traumatic SCI, standardized SCPP goal-directed care through LSAD monitoring for the first 5 days postinjury was feasible and without SCPP-related complications. Our institution is the first in the world to implement SCPP as a clinical standard of care, and this represents the first published report of the implementation. 
7:50 - 7:52 am

\section{Cervical Collar, Halo Brace and Surgery for Treatment of Type II Odontoid Fractures in the Elderly: A Markov Analysis}

Mark Andrew Attiah MD, MS, MBE; Jane Kim; Allison Portnoy; Andrew Schoenfeld; Luke Macyszyn MD, MA

Introduction: There is no consensus as to the most advantageous treatment strategy for acute type II odontoid fractures in patients 65 years and older without neurologic deficit.

Methods: We built a decision-analytic state transition model with health states defined by pain, complications, and development of delayed myelopathy. We estimated transition probabilities from published literature. Health outcomes were measured in quality-adjusted life years (QALYs). We conducted analyses over patients lifetimes. Health outcomes were discounted by $3 \%$ annually. We used probabilistic sensitivity analyses to account for uncertainty in data inputs.

Results: We estimated that the collar treatment strategy resulted in the greatest amount of QALYs, followed by halo bracing. Surgical options resulted in fewer QALYs, including surgical salvage following failure of bone union across the fracture after noninvasive options, due to decreased quality of life scores at 1 year, and a higher complication rate. The decrease in the already very low rate of developing delayed myelopathy did not compensate for these shortcomings. Probabilistic sensitivity analysis showed that collar resulted in the most QALYs in $60.1 \%$ of the simulations followed by surgery in $25.8 \%$ and halo in $13.6 \%$.

Conclusion: In patients 65 years and older with acute type II odontoid fracture without neurologic deficit, rigid cervical collar application is an attractive option compared with halo and surgery. This finding supports noninvasive treatment options for these patients. 
7:52 - 7:54 am

\section{Same Day Surgical Intervention Dramatically Minimizes Complication Occurrence for Central Cord Syndrome}

Cole Bortz BA; Avery Brown; Haddy Alas; Katherine Pierce; Sara Naessig; Burhan Janjua; Aaron Hockley MD; Alex Soroceanu; Daniel M. Sciubba MD; Bassel G. Diebo MD; Michael Gerling; Peter G. Passias MD

Introduction: As surgery is increasingly recommended for patients with neurologic deterioration secondary to central cord syndrome (CCS), it is important to investigate the relationship between time to surgery and patient outcomes. The merits of early vs delayed surgical treatment remain controversial in the literature.

Methods: CCS patients were isolated in NIS database 2005-2013. Operative patients were grouped by time to intervention: same day, 1-day delay, 2, 3, 4-7, 8-14, and >14 days. ANOVA and chi-squared tests compared demographics, Charlson Comorbidity ( $\mathrm{CCl}$ ) scores, LOS, discharge status, periop complications, and charges across patient groups. Controlling for age, $\mathrm{CCl}$, and concurrent traumatic fractures, binary logistic regression assessed surgical timing associated with increased odds of periop complication, using same-day as reference group(OR [95\% Cl]).

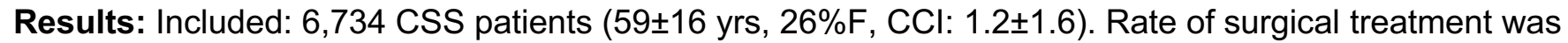
$64.1 \%$, with rates of surgery increasing from $2005(50 \%)$ to $2013(73 \%, p<0.001)$. The most common injury mechanisms were falls (30\%) and pedestrian accidents (7\%). Of patients that underwent surgery, $52.0 \%$ underwent fusion (62\% 2-3 levels, 33\% 8-levels, $1 \% 9+$ levels), 30\% discectomy, and $14 \%$ other decompression of the spinal canal. Differences in baseline characteristics and outcomes across patient groups: same-day patients had the lowest LOS, total charges, and the highest rates of home discharge. Sameday patients showed a trend of lower periop sepsis neurologic complications. Patients delayed $>14$ days to surgery had increased odds of periop cardiac (7.0 [1.6-30.0]) and infection (6.1 [2.2-16.3]) complications. Timing groups beyond 3-days showed increased odds of VTE: 4-7 days (3.0 [1.6-5.5]), 8-14 days (3.0 [1.46.3]), 14+ days (5.6 [2.3-13.6]).

Conclusion: CCS patients undergoing surgery the same day as admission had lower odds of complication, hospital charges, and higher rates of home discharge than patients that experienced a delay to operation. Delay $>14$ days to surgery was associated with inferior outcomes, like increased odds of cardiac complication and infection. 
7:54 - 7:56 am

\section{Predictors of Thoracic and Lumbar Spine Injuries in Patients with Traumatic Brain Injury: A Nationwide Analysis}

Blake M. Hauser ; John McNulty; Mark M. Zaki BS, BA; Saksham Gupta BA; David J. Cote BS; Joshua Bernstock MSc, MPH; Yi Lu MD, PhD; John Chi; Michael Groff; Ayaz M. Khawaja MD; Timothy R. Smith MD, $\mathrm{PhD}, \mathrm{MPH}$; Hasan A. Zaidi BS, MD

Introduction: Cervical spine injury screening is a common practice for patients with traumatic brain injury (TBI). However, risk factors for concomitant thoracolumbar trauma remain unknown.

Methods: We conducted a multi-center, retrospective cohort analysis of TBI patients in the National Trauma Databank from 2011-2014 using multivariable logistic regression.

Results: Out of 768,718 TBls, 46,654 (6.1\%) and 42,810 (5.6\%) patients were diagnosed with thoracic and lumbar spine fractures, respectively. Only $11 \%$ of thoracic spine fracture patients and $7 \%$ of lumbar spine fracture patients had an accompanying spinal cord injury at any level. The most common mechanism of injury was motor vehicle accident ( $67 \%$ of thoracic and $71 \%$ and lumbar spinal injuries).

Predictors of thoracic spine fractures included moderate (OR 1.26; 95\% Cl 1.21-1.31) and severe Glasgow Coma Scale (GCS) score (OR 1.71; 95\%Cl 1.67-1.75) compared to mild; epidural hematoma (EDH; OR 1.36; $95 \% \mathrm{Cl} 1.28-1.44)$; abdominal injury (OR 2.02; 95\% Cl 1.97-2.07); lower extremity injury (OR 1.38; 95\% Cl 1.351.41); upper extremity injury (OR 2.19; $95 \% \mathrm{Cl} 2.14-2.23)$; smoking (OR 1.09, $95 \% \mathrm{Cl} 1.06-1.12)$; and obesity (OR 1.39; 95\% Cl 1.34-1.45).

Predictors of lumbar spine fractures were similar, though with a stronger association with lower extremity and thoracic injuries and a weaker association with upper extremity injuries: moderate $(\mathrm{OR} 1.13 ; 95 \% \mathrm{Cl} 1.08-1.18)$ and severe (OR 1.17; 95\%Cl 1.13-1.20) GCS score compared to mild; EDH (OR 1.11; 95\%Cl 1.04-1.19); lower extremity injury (OR 2.50; $95 \% \mathrm{Cl} 2.45-2.55)$; upper extremity injury (OR $1.15 ; 95 \% \mathrm{Cl} 1.13-1.18)$; thoracic injury (OR 4.45; 95\%Cl 4.35-4.55); smoking (OR 1.12; 95\%Cl 1.09-1.15); and obesity (OR 1.29; 95\% Cl 1.241.35).

Conclusion: We identified GCS, smoking, upper and lower extremity injuries, and obesity as common risk factors for thoracic and lumbar spinal fractures for TBI. Abdominal injuries also predict thoracic fractures, while thoracic injuries predict lumbar fractures. 
7:56 - 7:58 am

\section{Results of an Institutional ED Protocol to Expedite MRI for Patients with Suspected Spinal Cord Compression}

Jay K. Nathan MD; Zoey Chopra; Paul Park MD; Mark E. Oppenlander MD

Introduction: In 2014, our institution began a protocol to identify patients at highest risk of spinal cord compression and obtain their MRIs within 4 hours. This protocol is labor-intensive for our neurosurgery service, requiring consultation prior to imaging, and may be over-utilized.

Methods: Retrospective review of ED patients treated under this protocol from July 2016 through June 2017, excluding acute trauma. Exam findings were separately recorded from ED and neurosurgery, as were relevant factors including history of cancer or spine surgery. Outcomes included MRI diagnosis of cord compression or cauda equina syndrome, and urgent treatment with surgery or radiation within 6 hours of imaging.

Results: 73 orders for 69 patients were analyzed. Region of clinical concern was most commonly lumbar $(48 \%)$ followed by thoracic (37\%). 22 MRIs were positive $(30.1 \%)$, yet 5 occurred after the 4 -hour goal. Chisquare testing revealed that a new inability to ambulate and sensory level on neurosurgery exam were independently associated with a positive MRI, with only sensory level remaining statistically significant on multivariate logistic regression $(P=0.006$, OR 6.74, 95\% Confidence Interval 1.7126 .57 ).

Following 11 of $73 \mathrm{MRIs}(15.1 \%)$, patients underwent urgent treatment. Weakness or sensory level on neurosurgery exam were each independently associated with this, though neither retained significance in multivariate analysis.

Abnormal sensory level alone had a positive predictive value of $69.2 \%$ for cord compression on MRI and $38.5 \%$ for urgent treatment.

Conclusion: This protocol had a false-activation rate of $70 \%$ for MRI diagnosis and $85 \%$ for subsequent urgent treatment, suggesting room for refinement to minimize overuse. A new inability to ambulate and abnormal sensory level were the two factors most directly associated with spinal cord compression and could be used as standalone triggers for activation. 
Spine Summit Meeting Abstracts, May 2021

7:58 - 8:00 am

\section{Targeting the $\mathrm{K}+/ \mathrm{Cl}-$ co-transporter KCC2 Facilitates Rapid Recovery after Acute Cervical Spinal Cord Injury}

Zachary A. Medress MD; Vanessa M. Doulames PhD; Adrian Rodrigues BA; Giles W. Plant PhD

Introduction: Spinal cord injury (SCI) results in a penumbra of damaged yet salvageable tissue that has been rendered functionally silent by the milieu of the injury micro-environment. KCC2, a neuron-specific potassium chloride antiporter responsible for maintaining low intracellular [Cl-], is significantly down-regulated in neurons following $\mathrm{SCl}$, leading to dysregulation of fast synaptic inhibition in damaged spinal cord circuits. We hypothesized that restoration of KCC2 function by delivering a small molecule KCC2 agonist following cervical $\mathrm{SCl}$ would augment functional recovery.

Methods: We administered the agent CLP290, a small molecule agonist of KCC2 that crosses the blood brain barrier, to rats 24 hours after undergoing a right unilateral spinal cord contusion at C5 (100 Kilodynes). The drug was administered daily via intraperitoneal injection $(35 \mathrm{mg} / \mathrm{kg})$ for six weeks after injury. Animals underwent testing of sensorimotor function, gait, and complex coordination. Immunohistochemical analysis was performed in order to determine the effect of KCC2 modulation on excitatory and inhibitory subsets of cervical inter-neurons.

Results: Within one week after injury, animals $(n=18)$ receiving daily intraperitoneal injection of CLP290 demonstrated significantly improved scores on a complex ladder walking task compared to vehicle-treated animals $(22.8 \%$ vs. $45.5 \%$ missteps, $p=0.0018)$. Automated gait analysis with CatWalk XT demonstrated improved swing speed cadence, and gait regularity in CLP290-treated animals four weeks after injury. Immunohistochemical analysis demonstrated alterations in KCC2 expression within populations of cervical inter-neurons in peri-lesional tissue in CLP290 treated animals.

Conclusion: For the first time, wedemonstrated that daily systemic administration of a small molecule KCC2 agonist improves functional outcomes after severe cervical contusion within the acute phase after SCl. Taken together, these results support KCC2 as a promising therapeutic target for cervical spine injury. Further translational studies investigating the safety, pharmacokinetics, and mechanism of CLP290 should be undertaken in order to further establish its potential as a treatment for human $\mathrm{SCl}$. 
8:00 - 8:02 am

\section{Conservative vs Surgical Management of Type II Odontoid Fractures: A Retrospective Analysis of Sixty-six Patients}

Nathan A. Shlobin ; Akash Mitra; Hooman Azad; Nikil Prasad BS; Michael Cloney MD, MPH; Nader S. Dahdaleh MD

Introduction: Type II odonoid fractures are the most common fractures of the axis. The management of these fractures remains a topic of interest especially in the elderly population. Both surgical stabilization and non operative treatments can be considered depending on the age, neurological status and fracture degree of biomechanical stability of the fracture.

Methods: A retrospective study reviewing the charts of66 patients who were treated for type II odontoid fractures from 2015-2019 were included in this study. Patient, event, and treatment characteristics were collected, including: demographics, Charlson Comorbidity Index $(\mathrm{CCl})$, cause, treatment (conservative vs. surgical) and outcome measureed by Nurick scores (Nuricks) at various times.

Results: Those treated surgically tended to be younger $(p=0.0001)$ and healthier $(p=0.005)$. Conservative therapy was pursued preferentially in fractures due to falls $(p=0.003)$. Both those treated conservatively and surgically had similar Nuricks at presentation $(p=0.48)$, however, at last follow-up those treated surgically had significantly lower Nuricks $(p=0.004)$ and greater improvement in Nuricks $(p=0.0001)$. To control for demographic differences between the surgically and conservatively managed groups a multivariate analysis was performed. This analysis demonstrated that only Nuricks at presentation $(p=0.016)$ and surgical management $(p=0.002)$ were significant predictors of change in Nurick at last follow-up.

Conclusion: Surgical intervention when controlled for age, $\mathrm{CCl}$, cause, and initial Nuricks was predictive of greater clinical improvement following a type II odontoid fracture. 
8:02 - 8:04 am

\section{Survival and Extension of Motor and Sensory Neuron Constructs (micro-TENNs) in Spinal Cord Injury}

Patricia E. Zadnik; Ahmed Albayar; Justin Burrell; Kevin Browne; Victoria Johnson; Douglas H. Smith MD; D. Kacy Cullen; Ali Kemal Ozturk MD

Introduction: Our group has developed micro-tissue engineered neural networks (micro-TENNs), a pioneering strategy to bridge axonal injuries. This technology utilizes neural circuits that can serve as a functional bridge to restore connectivity in addition to providing physical guidance for axons to traverse through the injured spinal cord. Moreover, micro-TENNs can be implanted using minimally invasive techniques, sparing the spinal cord from further injury.

Methods: Micro-TENNs were constructed using agarose hydrogel outer encasement lined with extracellular matrix (12 mm long, $700 \mathrm{~m}$ outer diameter, and $300 \mathrm{~m}$ inner diameter). Rat embryonal dorsal root ganglion (DRG) explants labeled with green fluorescent protein (GFP) or cortical motor neuron aggregates labeled with Td-Tomato were cultured within the inner lumen of the micro-TENN until a bridging axonal fascicle was noted. Female Sprague-Dawley rats were injured using a balloon-compression SCl model and allowed to recover for two weeks.

Micro-TENNs were implanted using stereotaxis along the longitudinal axis of the injury. Survival time-points were one-week (Sensory; $n=3$, Motor; $n=2$ ), one-month (Sensory; $n=7$, Motor; $n=5$ ) and three-month (Motor; $\mathrm{n}=4$ ) post-implantation of micro-TENNs. Acellular controls were implanted and sacrificed at similar time-points $(n=15)$.

Results: Five of seven DRG-micro-TENN implanted animals demonstrated axonal outgrowth at one-month post-implantation.

At one-week, no DRG-micro-TENN implanted animals revealed axonal outgrowth into host tissue, however, lumens of two constructs showed GFP positive axons. Motor micro-TENNs demonstrated axonal survival and regeneration at one-month and three-month time-points.

Conclusion: DRG and motor micro-TENNs survive manipulation and implantation for up to three-month postimplantation. Importantly, in both models, axonal sprouting was noted at one-month post-implantation. These findings suggest micro-TENNs as a potential multi-modal treatment strategy for SCl. 
8:04 - 8:06 am

\section{3-Dimensional Bioprinted Spinal Cord Scaffolds Encourage Neuronal Network Formation}

Nicholas Lavoie; Daeha Joung; Manuel Esguerra; Michael McAlpine; Ann M. Parr MD, PhD, FRCS(C);

Introduction: Chronic Spinal Cord Injury ( $\mathrm{SCl}$ ) currently has few treatment options. Encouraging results have been demonstrated utilizing human neural progenitor cell transplants but issues remain including survival, immune response, and lack of mechanistic understanding.

Methods: Regionally specific human spinal cord neural progenitor cells were generated from iPSCs utilizing a novel culture protocol developed in our laboratory. Cells were then 3D bioprinted in precise locations directly into user-defined scaffolds via one-pot printing. 30 scaffolds were cultured for 13-50 days and then examined for survival, differentiation, axon extension, and electrophysiological properties.

Results: Cells survived and differentiated into organotypically layered ventral neuronal cells. Cells in the scaffolds were shown to differentiate up to 19 days faster than with traditional 2D culture methods. They extended axons along the length of the channels, and displayed electrophysiological dynamics indistinguishable from spinal neurons in vivo.

Conclusion: 3D printed spinal cord scaffolds have several advantages over direct transplantation of cells including cell survival and more rapid differentiation, leading to improved neuronal network formation. Future work will involve transplantation of the scaffolds in vivo. 
8:06 - 8:08 am

\section{Traumatic Spinal Injuries from Border Wall Jumping at the U.S.-Mexico Border: A Single Institution's 6- Year Experience}

Wyatt L. Ramey MD; Peyton L. Nisson BS; Travis M. Dumont MD; R. John Hurlbert MD, PhD, FACS, FRCS(C)

Introduction: The border between the United States (US) and Mexico is an international boundary spanning $3000 \mathrm{~km}$, where unauthorized crossings occur regularly. We examine patterns of spine injuries, health care utilization, and financial costs at ourinstitution, which services over 350 perimeter miles of the U.S.-Mexico border, incurred by patients from wall-jumping into the US.

Methods: A review of all patients who suffered a spinal column injury as a direct resultof jumping over the U.S.-Mexico border wall was performed at a single Level-1 Trauma center between January 2012 to December 2017. Demographics, clinical status, radiographic findings, treatment, length of stay, and financial data were analyzed for all patients sufferingsuch injuries during that time.

Results: A total of 50 patient were diagnosed with traumatic spinalcolumn injury spanning this 6-year period. There were 28 males and 22 females, with a mean age of 34 years (Range 17-63 years). Twenty patients $(40 \%)$ underwent surgical fixation with 6 of those also requiring decompression. There were 5 acute spinal cord injuries (SCI); 3 ASIA A and 2 ASIA D SCI. The grand total of medical charges (summation of both provider and hospital charges) equaled $\$ 2,477,617$, while reimbursement totaled $\$ 459,735$ thus yielding a total reimbursement rate of $18.6 \%$.

Conclusion: Spinal column trauma can be a devastating injury for immigrants jumping over the U.S.-Mexico border wall. These injuries tended to be treated more aggressively (40\% undergoing surgical fixation/decompression) due to the presumed lack of follow-up. Significant costs, including physical, financial, and emotional, are associated with such injuries. Further efforts are needed from both Mexican and American entities to minimize risk to immigrants and support surrounding border healthcare systems. 
8:08 - 8:10 am

\section{Predictors of Neurological Improvement after Posterior Fusion Surgery in Degenerative Cervical Spine Disease}

Nida Fatima; John H. Shin MD

Introduction: Posterior cervical fusion surgery is a common treatment option to alleviate the radiculopathy and myelopathy associated with degenerative cervical spine disease (DCSD).

Methods: A retrospective chart review of the consecutive patients admitted at an academic tertiary care hospital was carried out from 2009-2019. For pre-operative demographics, surgical and radiographic characteristics, a univariable association with the post-operative clinical improvement was first determined using logistic regression, and a prediction model was then constructed using all characteristics significant at the 0.30 alpha level.

Results: The study cohort included 199 patients (50.8\% females, mean age:65.512.5 years), with a median clinical follow-up of 361 days. There was an overall clinical improvement in $51.3 \%(n=102)$ of the patients following the posterior fusion surgery. Patients with pre-operative modified Japanese orthopedic association (mJOA) score of 15-17 had 2.8-folds higher likelihood (OR:2.87,95\%Cl:1.29-6.37,p=0.01) of clinical improvement at the last follow-up evaluation after posterior fusion surgery. Furthermore, patients with preoperative mJOA score of $0-11$ had $93 \%$ less likelihood of clinical improvement at the last follow-up evaluation (OR:0.07,95\% Cl:0.01-0.36, $\mathrm{p}=0.001$ ). Furthermore, patients with pre-operative C2-C7 SVA4.5 cm had 4.1folds greater odds of clinical improvement at last follow-up evaluation (OR:4.19,95\%Cl:1.65-10.5, $\mathrm{p}=0.002$ ).

Conclusion: Our results corroborate an overall improvement in clinical status following posterior fusion surgery in DCSD, and pre-operative neurological status and C2-C7 SVA are independent predictors of clinical improvement at the last follow-up. 
8:10 - 8:12 am

\section{Analysis of Transfusions and Effect of Anemia on Transfusion Rates in Common Elective Spine Surgery Procedures}

Yaroslav J. Gelfand MD; Rafael De la Garza Ramos MD; Michael Longo BA; Vijay Yanamadala MD, MBA; Reza Yassari MD, MS

Introduction: Pre-operative anemia along with perioperative blood loss and transfusions have been linked to an increased rate of complications and length of stay. Comparison of transfusion risks and the effect of degree of anemia on transfusion risks in various elective spine procedures is poorly studied.

Methods: Patients undergoing elective spine surgery were identified in the American College of Surgeons National Surgical Quality Improvement Program (ACS-NSQIP) database from 2006 to 2016. Those with disseminated cancer, spine metastasis, and epidural abscess were excluded. 12 different groups based on the procedure were identified. Minimum overlap between the groups was confirmed. The descriptive statistics was used to analyze the transfusion risk and the effect of anemia (hematocrit less than $40 \%$ in males, and less than $36 \%$ in females) and severe anemia (hematocrit less or equal to $29 \%$ ) on transfusion rates for each individual procedure.

Results: 172,753 patients fit the inclusion criteria. Transfusions happened in $5.9 \%$ of the patients analyzed, with smallest number happening in ACDF patients $(0.2 \%)$ and largest number in deformity surgery $(46.1 \%)$. $15.8 \%$ of all patients had preoperative anemia, and $0.6 \%$ had severe anemia. Anemia and severe anemia had independent significant effect on transfusions in all procedures (even when controlled for operative time and patients age and gender), but the effect varied widely between the procedures.

Conclusion: This is the largest study evaluating the effect of various degrees of anemia on the transfusion risks in common spine surgery procedures. Given that most hospitals have limited resources and ERAS protocols becoming an integral part of spine care, this study allows for selection of patients and procedures that would benefit the most from pre-operative anemia workup and optimization. 
8:12 - 8:14 am

\section{Inter-Observer Reliability of the Modified Japanese Orthopedic Association Score in Degenerative Cervical Myelopathy}

Allan R. Martin BASc, MD; Jamie RF Wilson BA.BM.BCh(OXON).FRCS(Neurosurgery); Thorsten Jentzsch; Fan Jiang MD; Jetan H. Badhiwala; Ali Moghaddamjou; Muhammad Akbar BSc, MD; Anick Nater MD, PhD; Anna Rienmuller; Mario Ganau MD, PhD, FACS; Eric M. Massicotte MD; Michael G. Fehlings MD, PhD, $\operatorname{FRCS}(\mathrm{C})$, FACS

Introduction: The modified Japanese Orthopedic Association (mJOA) score is widely accepted as the most important assessment in degenerative cervical myelopathy (DCM); this score has been utilized in clinical practice guidelines and directly influences treatment recommendations, but its reliability has not been established.

Methods: This prospective cross-sectional study involved blinded administration of a refined version of mJOA to DCM patients by 2 or more experienced clinicians. The reliabilities of subscores and total score were analyzed using intra-class correlation (ICC) and concordance. Subgroup analyses were performed by mJOA severity (mild: 15-17, moderate: 12-14, severe: <12). Data were also analyzed using ANOVA for differences by assessor, assessment order, previous surgery, age, and sex.

Results: 115 DCM patients underwent 245 assessments. ICC was 0.66 for upper extremity motor, 0.70 for lower extremity motor, 0.57 for upper extremity sensation, 0.65 for sphincter function, and 0.71 for total mJOA. The average difference in mJOA was 0.90 points between assessors. Identical scores (across all 4 subscores) were observed in $21 \%$, differences of $>=2$ points occurred in $19 \%$, and disagreement between mild and moderate severity occurred in $14 \%$ of patients. Lower extremity motor score was lower during $2^{\text {nd }}$ assessments, $p=0.02$ ). Other variables that were analyzed did not demonstrate significant relationships with mJOA scores.

Conclusion: The inter-observer reliability of the mJOA is moderate, and disagreement occurs in the vast majority of patients. These findings suggest that the mJOA should be interpreted with caution and considered in conjunction with additional measures; when the score falls near the threshold between severity categories, or when a patient is monitored longitudinally for deterioration, small differences can alter management. Further efforts to standardize the mJOA are needed to improve its reliability and help deliver optimal management of DCM. 
8:14 - 8:16 am

\section{Relationship Between Gastrointestinal Comorbidities, Clinical Presentation and Surgical Outcome in Patients with Degenerative Cervical Myelopathy: Analysis of a Global Cohort}

Aria Nouri MD, MSc; Jetan H. Badhiwala; So Kato; Hamed Reihani-Kermani MD; Kishan Patel; Jefferson R. Wilson MD, PhD; Insa Janssen MD; Enrico Tessitore; Karl Schaller MD; Joseph S. Cheng MD, MS; Michael G. Fehlings MD, PhD, FRCS(C), FACS

Introduction: Degenerative Cervical Myelopathy (DCM) is the most common cause of spinal cord impairment in adults, presenting most frequently in patients 50 years or older. Gastrointestinal comorbidities (GIC) commonly occur in this group, however, their relationship with DCM has not been thoroughly investigated.

Methods: 757 patients with clinical data and 458 with MRI data from2 AOSpine International studies on DCM were evaluated. GIC wererecorded at presentation and included gastric, intestinal, hepatic and pancreatic conditions. Patients were dichotomized into 2 groups: GIC and without GIC. Clinical and MRI presentation, and baseline neurological and functional status were compared. Neurological and functional outcome at 2-years follow-up were also compared.

Results: GIC were present in 121 patients (16\%). These patients were less commonly male (48.76\% vs $65.4 \%, p=0.001)$, were slightly less neurologically impaired based on Nurick grade (3.05 \pm 1.10 vs $3.28 \pm 1.16$, $p=0.044)$ but not based on mJOA (12.74 \pm 2.62 vs $12.48 \pm 2.76, p=0.33)$, had a worse general heath score (71.27 \pm 16.88 vs $75.22 \pm 17.08, p=0.022)$, and worse neck disability $(46.31 \pm 20.04$ vs $38.23 \pm 20.44, p<0.001)$, a high rate of psychological comorbidities $(31.4 \% \mathrm{vs} 10.4 \%, \mathrm{p}<0.001)$ and a lower prevalence of upper motor neuron signs (hyperreflexia, $70.2 \%$ vs $78.9 \%$, $p=0.037$; Babinski's sign $24.8 \%$ vs $37.3 \%$, $p=0.008$ ). On MRI, GIC patients less commonly exhibited signal intensity changes (T2 hyperintensity, $49.2 \%$ vs $75.6 \%, p<0.001$; T1 hypointensity, $9.7 \%$ vs $21.1 \%, p=0.036)$ and had a lower number of T2 hyperintensity levels $(0.82 \pm 0.98$ vs $1.3 \pm 1.11, p=0.001)$. There was no difference in surgical outcome between the groups.

Conclusion: DCM patients with GICs are more likely to be female, have psychological comorbidities and have significantly more general health impairment and neck disability. However, these patients have less clinical and MRI features typical of more severe neurological impairment. This constellation of symptoms is considerably different than those typically observed in DCM and it is therefore plausible that nutritional factors including B12 deficiency which frequently manifests in elderly patients may contribute to this unique observation. 
8:16 - 8:18 am

\section{Predictors of the Timing of Decompressive Surgery in Metastatic Spinal Cord Compression}

Nida Fatima; John H. Shin MD

Introduction: The optimal surgical timing in patients with metastatic spinal cord compression (MSCC) is still controversial, and the previous studies did not clearly discern the predictors of the timing of decompressive surgery (DS) in patients with MSCC.

Methods: Consecutive patients with MSCC presented at an academic tertiary care hospital from 2011 to 2018 were identified through retrospective chart review. For the timing of the surgery categorized as Group 1:<24 hours ( $n=40,33.6 \%$ ), Group 2:24-48 hours ( $n=28,23.5 \%)$, and Group3: $>48$ hours $(n=51,42.9 \%)$ from the onset of acute neurological symptoms, univariable association with patient characteristics were first determined, and a prediction model using Proportional Cox Hazard Regression Model was constructed using all characteristics significant at the 0.20 alpha level.

Results: The study cohort included 119 patients (63\% males, median age 67 years), with a median survival of 345 days (95\% $\mathrm{Cl}$ : 91.7-652.8 days, $\mathrm{p}=0.15)$. Postoperative changes in neurology showed statistically significant improvement in the patients treated within 24 hours compared to the other groups $(p=0.003)$. Multivariable regression analysis revealed that pre-operative poor neurological status (modified Frankel Grade A-B-C) was a significant independent predictor of performing DS within 24 hours by 14.7 folds (HR:14.7, 95\%Cl:2.01-107.9,p=0.008). Similarly, the pre-operative poor Karnofsky performance status (KPS) (>80) was significantly associated with DS <24 hours in patients with MSCC by 2.6 folds (HR: $2.61,95 \% \mathrm{Cl}: 1.12-$ $6.03, p=0.02$ ).

Conclusion: Our results suggest that poor pre-operative neurology serve as a surrogate marker to adjudicate timing of the DS in patients with MSCC. 
8:18 - 8:20 am

\section{Validation of 5-Item Modified Frailty Index for Patients following Posterior Cervical Fusion Surgery}

Nida Fatima; John H. Shin MD

Introduction: The relationship between frailty and postoperative outcomes following degenerative cervical spine has been studied widely.

Methods: A total of 3,210 patients from the American College of Surgeons, National Surgical Quality Improvement Program who underwent PCF between 2005 and 2017 were extracted. Multivariable logistic regression analysis (MLR) model, after adjusting for age and smoking status, was computed to assess the association between frailty indices and outcome parameters. Assessment of agreement between the 5- and 11-item frailty index was achieved using Kappa statistics.

Results: MLR model of postoperative 30-day morbidity with an exposure score of $\geq 2$ indicated that $\mathrm{mFI}-5$ (OR: $3.04,95 \% \mathrm{Cl}: 1.36-6.77, \mathrm{p}=0.007$ ) which is comparable to $\mathrm{mFI}-11$ (OR: $3.42,95 \% \mathrm{Cl}: 1.22-9.67, p=0.02)$. The secondary end-points with exposure of $\geq 2$ factors in terms of length of hospital stay [mFI-5 $(\mathrm{OR}: 1.12,95 \% \mathrm{Cl}$ : 1.06-1.17, $\mathrm{p}<0.001$ ) vs mFl-11 (OR: $1.11,95 \% \mathrm{Cl}: 1.05-1.17, \mathrm{p}<0.001)$ ], 30-days reoperation [mFI-5 (OR: 1.34 , 95\%Cl: $0.37-4.81, p=0.65$ ) vs $\mathrm{mFl}-11$ (OR: $1.75,95 \% \mathrm{Cl}: 0.37-8.23, \mathrm{p}<0.47$ )], and mortality [mFI-5 (OR: 5.51 , 95\% Cl: $0.33-90.8, p=0.23)$ vs mFI-11 $(p=0.99)]$. The scoring agreement between the 5 -item and the 11-item indices were strong with a weighted kappa of $0.41(p<0.001)$.

Conclusion: The 5-item $\mathrm{mFI}$ is a good predictor of postoperative outcomes while allowing for a significant simplicity compared to 11 -item $\mathrm{mFI}$. Thus 5 -item $\mathrm{mFI}$ is a useful research tool for identifying patients at increased risk of postoperative morbidity following posterior cervical spinal fusion surgery. 
8:20 - 8:22 am

\section{Influence of Socioeconomic Disparities on Management and Outcomes After Traumatic Upper Cervical Spine Fracture}

Aladine A. Elsamadicy BE; Erica Giles; Wyatt David; Andrew B. Koo ; Adam Kundishora MD; Christopher Sungwoon Hong MD; Kristopher T. Kahle MD, PhD; Michael DiLuna

Introduction: There is a paucity of data examining the impact socioeconomic disparities, such as race and insurance status, have on management and treatment outcomes following upper cervical spine (UCS) fracture.

Methods: The medical records of 239 consecutive cases of patients presenting with an UCS fracture at a major academic institution from 2002 to 2013 were retrospectively reviewed. Patients were grouped by race (white and nonwhite) and insurance (government, private, no insurance). Demographic information, Charlson Comorbidity Index $(\mathrm{CCl})$, injury information, primary management and outcome data at follow-up were collected.

Results: There were 218 patients included in this study, comprised of $110(50.5 \%)$ females with a mean patient age at injury of $69(\mathrm{SD}=21.6)$. There were $79(36.2 \%)$ patients with a $\mathrm{CCl}$ score of $0,57(26.3 \%)$ with score of 3 or greater, and $9(4.2 \%)$ score over 5 . On logistic regression, the Nonwhite cohort was more likely to have complete healing [OR: $2.80,95 \% \mathrm{Cl}:(1.00,7.87), p=0.05]$ and lower likelihood of delayed healing [OR: $0.22,95 \% \mathrm{Cl}:(0.05,1.00), p=0.04]$ compared to the White cohort. Between the Uninsured vs. Non-Insured Cohorts, the Uninsured cohort was more likely to undergo definitive halo [OR: 3.79, 95\%: (1.30, 11.02), $p=0.01]$ Compared to the Private coverage cohort, the Medicare/Medicaid cohort had lower likelihood of follow-up CT [OR: 0.40, 95\% Cl: $(0.17,0.95), p=0.03$ ] and increased likelihood of delayed healing [OR: 3.50, 95\% Cl: $(0.95,12.86), p=0.05$. The Medicare/Medicaid cohort had decreased likelihood of complete healing [OR: $0.28,95 \% \mathrm{Cl}:(0.10,0.77), p=0.01]$.

Conclusion: Our study suggests that socioeconomic differences including race and insurance may have a significant impact on the management, resource use, and post-treatment outcomes for patients presenting with upper cervical spine fracture. 
8:22 - 8:24 am

\section{Role of Cervical Collar on Outcomes Following Cervical Laminectomy and Fusion: CSM-S Trial Sub- group Analysis}

Mehdi Khani MD; Melissa Dunbar; Robert G. Whitmore MD; Zoher Ghogawala MD, FACS; Subu N. Magge MD

Introduction: The ongoing multi-center prospective, randomized CSM-S trial examines ventral versus dorsal surgical strategies for cervical spondylotic myelopathy (CSM). One arm, dorsal laminectomy plus fusion, included patients whose post-operative collar usage varied by enrolling site.

Methods: Patients randomized to dorsal CSM surgery operated by posterior laminectomy/fusion were included. Group C: posterior laminectomy and fusion with collar and Group NC: posterior laminectomy and fusion without collar. Average collar use was nearly 2 months. Data compared included number of levels fused, 3 month and 1 year neck disability index (NDI), ongoingopioid use at 1 year, complications with 1 year of surgery, and time to return to work.

Results: Group C consisted of 39 patients from 10 centers (age range 47-81 years, mean 63.5 years) and Group NC consisted of 27 patients from 8 centers (age range 45-78 years, mean 61.3years). Average stenotic levels in Group $\mathrm{C}$ and $\mathrm{NC}$ was 3 and 2.59, respectively $(P=0.04) .3$ month NDI average in Group $\mathrm{C}$ and NC was $34.4 \%$ and $31.14 \%$, respectively $(P=0.48)$. One-year NDI average in Group C and NC was $23.44 \%$ and $28.48 \%$, respectively $(P=0.38) .17 .9 \%$ of patients in Group $C$ were still on opioids at 1 year, compared to $11 \%$ of patients in group NC ( $\mathrm{P}=0.51)$. Time to return to work average in Group $\mathrm{C}$ and NC was 5.04months and 4.97 months, respectively $(P=0.96)$. Percentage of complications within 1 year in Group $\mathrm{C}$ and NC was $33 \%$ and $22 \%$, respectively $(\mathrm{P}=0.32)$.

Conclusion: Use of cervical orthrosis postoperatively in CSM patients undergoing posterior laminectomy and fusion does not significantly alter outcome. 
8:24 - 8:26 am

\section{Which MRI Findings are Associated with Long-term Disability?}

\section{Peter Udby; Leah Carreon}

Introduction: Multiple MRI classifications are used to assess lumbar degenerative disease. The most widely used are the Pfirmann classification, the presence or absence of Modic Change (MC) and the Fujiwara classification of facet joint degeneration. The purpose of this study was to examine the associations between long-term RMDQ and baseline Pfirmann classification, Modic Changes (MC) and Fujiwara classification.

Methods: In 2004-2005, patients aged 18-60 with daily LBP were enrolled in an RCT and lumbar MRI was performed. Patients completed numeric rating scales (NRS, 0-10) for LBP and leg pain (LP), Roland-Morris Disability Questionnaire (RMDQ) at baseline and 13-years after the MRI. We performed a linear regression to determine associations with long-term disability (13-yr RMDQ). The MRI parameters included baseline disc degeneration (Pfirmann grade $>3$ on any lumbar level), Modic changes and facet joint degeneration (Fujiwara grade $>2$ on any lumbar level). Demographic variables at baseline included BMI, alcohol consumption, smoking and weekly physical activity.

Results: Of 204 cases with baseline MRI, 170 (83\%) were available for follow-up. 88 had disc degeneration $(52 \%), 67$ had $\mathrm{MC}(39 \%)$ and 86 had facet joint degeneration $(81 \%)$. The presence of MC was the only radiologic finding that was statistically significantly associated with better 13-year RMDQ scores $(p=0.003)$. Baseline disc degeneration and the presence of facet joint degeneration showed no significant association with 13-year disability. Baseline weekly physical activity was also significantly associated with better 13-year RMDQ scores $(p<0.000)$.

Conclusion: Presence of MC and weekly physical activity was significantly associated with less long-term disability. Baseline MRI findings of disc degeneration and facet joint degeneration were not associated with long-term disability. 
8:26 - 8:28 am

\section{Complications and Survival in Octogenarians Undergoing Surgery for Spinal Metastases with Instrumented Stabilization}

Ibrahim Hussain; Lily McLaughlin ; Ilya Laufer MD; Mark H. Bilsky MD; Ori Barzilai MD

Introduction: Octogenarians living with spinal metastases are a challenging population to treat, given a high incidence of medical comorbidities.

Methods: A retrospective study of patients aged 80 or greater years undergoing surgery for spinal metastases was performed. Cox proportional hazards and logistic regression were used for statistical analyses.

Results: 78 patients (mean 83.6 years) were included. 63 patients were treated by open decompression plus stabilization, the remaining by MIS pedicle screw stabilization. Average operative time, blood loss, and length of stay were 157 minutes, $615 \mathrm{~mL}$, and 9 days, respectively. Discharge destinations were home $(\mathrm{N}=39)$, rehab $(\mathrm{N}=32)$, and hospice $(\mathrm{N}=3) .4$ patients died in the hospital. Average survival was 136 days. 24 patients developed postoperative complications: wound dehiscence/infection $(\mathrm{N}=6)$, pulmonary $(\mathrm{N}=4)$, and hematoma $(\mathrm{N}=3) .9$ patients had return to the OR for complication management. There was a non-significant trend towards increased mortality when decompression was performed in addition to stabilization (HR=2.12; $P=0.09)$. There were also non-significant trends towards increased mortality $(H R=1.93 ; P=0.10)$ and complications $(\mathrm{OR}=2.08 ; \mathrm{P}=0.29)$ in surgeries involving the cervical/cervicothoracic spine. $E B L$ was significantly associated with increased risk of postoperative complications $(O R=1.001 ; P=0.048)$.

Conclusion: Surgery for metastatic spine disease in octogenarians should not be ruled out based on age only as it can be performed with goals of palliation. Octogenarians undergoing surgery for spinal metastases are at increased risk for postoperative complications particularly when these include cervical/cervicothoracic surgeries, decompression in addition to stabilization, and increasing intraoperative blood loss. 
8:28 - 8:30 am

\title{
Real Time Cervical Spine MRI for the Assessment of Dynamic Spinal Instability
}

\author{
Basem I. Awad MD; Khaled Zaghloul; Donia Sobh
}

Introduction: There is a large agreement that cervical spine instability (CSI) can be essentially diagnosed with clinical judgments e.g. intolerance to prolonged static postures, fatigue and inability to hold head up, better with external support, episodes of acute sharp pain with sudden movements.

Objective criteria have been established in the analysis of end-range flexion and extension radiographs to diagnose CSI. However, radiographs do not provide information about the quantity or quality of motion in the neutral zone, which limits their value in the diagnosis of CSI.Even after severe whiplash injuries, plain radiographs are usually normal despite clinical findings indicating presence of soft tissue damage.

Methods: Tencervical spine(of healthy participants) were scanned during neck flexion and extension maneuvers. Using 1.5T MRI scanner (Philips Ingenia), sagittal and axial T1 and T2 images of the cervical spine were obtained in routine neutral position. Real time MRI was performed in the sagittal plane using a balanced fast-field echo (BFFE) sequence with the patient slowly moving his/her neck to flexion and extension.

Results: Functional imaging technology, as opposed to static standards, is superior toillustrate the normal physiological changes during movement. Cervical spine dynamic imagingcan providequantitative measures relevant to evaluatethe presence and degree of dynamicCSI.

Conclusion: This pilot study demonstrates thatreal-time cervical spineMRlof the moving neck is feasible. Real Time MRI canbe an objective diagnostic tool for dynamic CSI. This technique is at an early stage of development but encouraging. 
8:30 - 8:32 am

\section{A Feasible Benefit Of The Renin-Angiotensin System Blockage: Looking For Clues In Cervical Spondylotic Myelopathy}

Alexander Perdomo-Pantoja MD; Alejandro Chara; Samuel Kalb; Joshua Casaos BS; Ali Karim Ahmed BS; Zachary Pennington; Ethan Cottrill BS; Sohan Shah; Bowen Jiang MD; Amir Manbachi PhD; Corinna C. Zygourakis MD; Timothy F. Witham BS, MD; Nicholas Theodore MD

Introduction: In vivo studies suggest a beneficial effect of renin-angiotensin system (RAS) blockers in the central nervous system, especially in brain inflammation and stroke. Data on their influence on the spinal cord is limited to a couple of rat models of spinal cord injury $(\mathrm{SCl})$ that have reported encouraging findings.

Methods: Adults with symptomatic degenerative cervical stenosis who underwent surgery were included. Demographic data, comorbidities, and antihypertensive medications (particularly RAS blockers) were collected. Functional status was evaluated using the modified Japanese Orthopedic Association [mJOA] and Nurick grading scales, while imaging characteristics (eg, canal and spinal cord compromise, increased signal intensity, signal intensity ratio, etc.) were measured on sagittal T2-weighted (T2W) MRIs.

Results: Of the 267 patients, 149 patients (55.8\%) had hypertension, of which 142 (95.3\%) were taking antihypertensive medications: 37 angiotensin-II receptor blockers [ARBs], 44 angiotensin-converting enzyme inhibitors [ACEIs], and 61 other medications).

Patients treated with ARBs displayed a higher signal intensity ratio (ie, lower signal intensity change in the compressed cord area) compared to untreated patients without hypertension $(p=0.004)$. Patients with hypertension had worse preoperative mJOA and Nurick scores than those without $(p<0.001)$. In the multivariate analysis, ARBs remained an independent beneficial factor for lower signal intensity change on T2W images $(p=0.04)$, while hypertension remained a risk factor for worse preoperative neurological status $(p<0.01)$.

Conclusion: In our study, cervical spinal cord compression patients with hypertension who were prescribed with RAS inhibitors had decreased T2W signal intensity change than untreated patients without hypertension. Prospective case-control studies may deepen understanding of RAS modulators in the imaging and functional status of chronic spinal cord compression. 
8:32 - 8:34 am

\section{Associated Risk Factors for Extended Length of Stay Following PCF for Cervical Spondylotic Myelopathy}

Aladine A. Elsamadicy BE; Andrew B. Koo ; Wyatt David; Megan Lee; Adam Kundishora MD; Ramana Gorrepati MD; Gregory A. Kuzmik MD; Joaquin Camara-Quintana; Luis Kolb MD; Maxwell Laurans; Khalid M. Abbed MD

Introduction: Relatively little is known about the clinical factors that predict extended length of hospital stay (LOS) for patients undergoing posterior cervical fusion (PCF) for cervical spondylotic myelopathy.

Methods: The National Inpatient Sample database was queried to identify adult patients ( $\geq 18$ years) with a diagnosis of cervical spondylotic myelopathy undergoing PCF between 2010 and 2014. Updated trend weights were used to assess patient demographics, comorbidities, complications, LOS, discharge disposition and total cost. Multivariate logistic regression was used to determine the odds ratio for risk-adjusted LOS.

Results: We identified 58,098 patients with 11,498 (24.7\%) experiencing an extended LOS. The Extended LOS cohort was older than the Normal LOS, and had similar proportion of female patients. The overall complication rates in the extended LOS cohort were 3-folds greater compared to the Normal LOS cohort (19.9\% vs. $60.5 \%, p<0.001)$. The total cost of admission was significantly greater in the extended LOS cohort $(\$ 29,719 \pm 14,835$ vs. $\$ 58,277 \pm 38,031, p<0.001)$. On multivariate logistic regression, several risk-factors were associated with extended LOS, including: female sex, Black and Hispanic race, healthcare coverage, deficiency anemias, rheumatoid arthritis/collagen vascular diseases, congestive heart failure, chronic pulmonary disease, coagulopathy, depression, hyperlipedmia, fluid and electrolyte disorders, paralysis, smoking, blood transfusion, and number of complications. The odds ratio for extended LOS was $2.47(95 \% \mathrm{Cl}$, 2.16-2.82) for patients with 1 complication and 8.99 (95\% Cl:7.69-10.50) for patients with >1 complication.

Conclusion: Our study demonstrates that patient baseline demographics, comorbidities, and post-operative complications all contribute to extended LOS after posterior cervical fusion for cervical spondylotic myelopathy. 


\section{8:34 - 8:36 am}

\section{Institutional Review of the Management of Type II Odontoid Fractures: Associations and Outcomes with Fibrous Non-union}

Christopher Wilson MD; Mariana Hoyos; Andrew Huh; Stephen Avila; Stephen K. Mendenhall MD; Miracle C. Anokwute BS, MD; George Eckert; David Stockwell MD

Introduction: Type II odontoid fractures may be managed operatively or nonoperatively. If managed with bracing bony union may never occur despite stability, which is termed fibrous nonunion.

Methods: A retrospective review of our spine trauma database was performed for adults with type II odontoid fractures between 2015-2019. Two-sample t-tests and Fishers Exact tests identified associations with follow-up stability and compared operative and non-operative outcomes. Sensitivity, specificity, and predictive values were calculated to validate initial stable upright cervical radiographs related to follow-up stability.

Results: Among 88 patients, 10\% received upfront surgical fixation, and 90\% were managed nonoperatively of whom $22 \%$ were unstable on follow-up. Associations with instability after nonoperative management include dens displacement $2 \mathrm{~mm}(\mathrm{OR} 0.29,95 \% \mathrm{Cl} 0.07-1.0)$, cerebrovascular disease (OR0.23, 95\% $\mathrm{Cl} 0.06-1.0)$ and myelopathy (OR0.04, 95\% Cl 0.0-0.92). Advanced age was not associated with follow-up instability. Stable initial upright radiographs were associated with stability on follow-up (OR 4.29, 95\% Cl 1.0-18) with excellent sensitivity and positive predictive value (Sensitivity $89 \%$, Specificity 35\%, PPV 83\%, NPV 46\%). Respiratory failure requiring ventilation was commonest in surgically managed patients $(33 \%$ vs. $3 \%$, respectively; $p$ 0.007). Operative or nonoperative management conferred no difference in length of hospital or intensive care stay, discharge disposition, and mortality.

Conclusion: We identified factors related to follow-up instability of type II odontoid fractures, compared morbidity of operative and nonoperative management, and validated use of upright cervical radiographs to assess type II odontoid fracture stability. 
8:36 - 8:38 am

\section{Predicting Survival in Patients with Metastatic Renal Cell Carcinoma: Which Scoring System is the most Accurate?}

Elie Massaad; Nida Fatima; Ganesh Shankar MD, PhD; John H. Shin MD

Introduction: Renal cell carcinoma (RCC) is one of the most commonly diagnosed malignancies, with an estimated 74,000 new cases in 2019 . Approximately $40 \%$ of bony metastasis occurs in the spine.

Methods: A multi-centric retrospective study included $n=86$ patients with spinal metastatic RCC. Preoperative scores were calculated using: (1) Tomita, (2) original Tokuhashi, (3) revised Tokuhashi, (4) original Bauer, (5) modified Bauer, (6) Katagiri, (7) Van Der Linden, (8) SORG classic algorithm, (9) SORG nomogram, (10) New England spinal metastasis score (NESMS). Univariate Cox proportional hazard models were calculated to assess the association of patient variables with 1-year survival. The time-dependent ROC was performed for each model. Cut-offs for (AUC) are as follows: excellent ( $A \cup C \geq 0.90$ ), good ( $A \cup C \geq 0.80$ and $<0.90$ ), fair (AUC $\geq 0.70$ and $<0.80$ ), and poor performance (AUC < 0.70).

Results: $\mathrm{N}=86$ patients $(60.90 \pm 11.36$ years, $73.25 \%$ male) undergoing spine surgery for spinal metastatic RCC . Univariate analysis showed that patient and tumor factors were strongly associated with 1-year survival: (1) Poor KPS (HR:6.78 [95\% Cl:1.96-23.48]), (2) ECOG grade 3-4 (HR:3.52 [1.57-7.91]), (3) Frankel grade AD (HR:2.54 [1.01-6.46]), (4) albumin <3.5 g/dL (HR:4.05 [1.67-9.80]). NESMS had the best performance in predicting survival at $\mathrm{t}=3$ months $(\mathrm{AUC}=0.83[95 \% \mathrm{Cl}: 0.63-1]), \mathrm{t}=6$ months (AUC=0.84 [0.70-0.98]), $\mathrm{t}=12$ months (AUC=0.88 [.77-0.99]). All other prognostic scores had a poor or fair performance at $t=3$ months (AUC range: $0.61-0.78), t=6$ months $(A \cup C=0.59-0.73), t=12$ months $(A \cup C=0.60-0.76)$.

Conclusion: Most validated prognostic scores have a poor or fair performance in predicting the survival of RCC surgical patients with spinal metastatic disease. Tumor-specific factors and newer treatment modalities affect the survival of RCC patients and should be evaluated in future prognostic studies 
8:38 - 8:40 am

\section{Effects of Prophylactic Hypothermia in Evoked Motor Potentials after latrogenic Spinal Cord Impact Injury}

Ahmed Jorge PhD; Souvik Roy; Erika Fish; Edward Dixon; David Hamilton; Jeffrey Balzer; Parthasarathy

Thirumala MD

Introduction: Rates for an iatrogenic spinal cord injury (iSCl) during spinal cord procedure range from $0.57 \%$ to $5.93 \%$ and can be caused by direct instrumentation, compressive injury during spinal correction, or vascular injury. It has been hypothesized that hypothermia may serve a neuroprotective role within iSCI and thus minimize morbidity in this population.

Methods: 26 Female Sprague Dawley rats were randomly assigned to a normothermic (control) group maintained at $36^{\circ} \mathrm{C}$ and a hypothermic group (experimental) maintained at $32^{\circ} \mathrm{C}$. A laminectomy was performed to all groups at the T8 level and subgroups were randomly assigned a contusive spinal cord injury with $200 \mathrm{kdyn}$ of force. Rats were then rewarmed after two hours of hypothermic treatment, with the sham surgery group experiencing the same procedure minus the contusion. Motor Evoked Potentials were obtained at baseline and at specified points after the procedure.

Results: When recording from the left lower limb, the hypothermic group had a significantly smaller decline in MEP amplitude compared to the normothermic group both at time of impact (mean difference $=1.3 u \mathrm{~V}, \mathrm{p}$ val<0.05) and 2 hours after (mean difference=1.6, $\mathrm{p}$-val<0.05). The hypothermic group continued to show higher MEP amplitudes when measured 35 days after the impact when compared to normothermic cohort (mean difference $=4.2 \mathrm{uV}, \mathrm{pp}$-val<0.05). There was no difference in MEP amplitudes between both groups at any stage when measuring from the forelimbs of the same rats.

Conclusion: MEPs were significantly different after an iSCI depending if hypothermia was applied. Moreover, hypothermia was shown to provide a protective effect as shown by higher MEPs immediately after, 2 hours after the injury and 35 days after injury. 
8:40 - 8:42 am

\section{Spinal Cord Injury in the United States Army Special Forces}

Remi A. Kessler BA; Raj K. Shrivastava MD; Ansh Bhammar; Jonathan J. Rasouli MD; Deborah L. Benzil MD, FACS, FAANS; Joshua B. Bederson MD; Constantinos G. Hadjipanayis MD, PhD

Introduction: Spinal cord injury (SCI) is high priority in military medicine, as $\mathrm{SCl}$ may be present in $>38 \%$ of all US war-related casualties. Despite that US Army Special Forces (SF) soldiers, known as Green-Berets, constitute $60 \%$ of all US Special Operations casualties, the demographics and ramifications of SCl specifically among SF have never been investigated.

Methods: A 162-question study was formulated via collaboration of neurosurgeons, SF-members, and GreenBeret Foundation (GBF) staff. Data on military background, medical history, and training/deployment were collected. This study was disseminated to thousands of soldiers via GBFs military network. Inclusion criteria mandated SF qualification (18-Series).

Results: 529 participants met inclusion criteria. SCI diagnosis was reported by $>18 \%$ of SF soldiers, of whom $84 \%$ were in combat and $51 \%$ are Military Free-Fall-qualified. $69 \%$ receive SCI disability benefits with mean Veterans-Administration (VA) disability rating of 82/100. Most common causes were Parachute Jump/Fastroping/Airborne-Operations (61\%). Over $88 \%$ were wearing headgear and only $36 \%$ were wearing body army at injury. Only $21 \%$ of SF soldiers with SCI were Medevac'ed.

Conclusion: Our study demonstrates high SCI incidence among US Army SF soldiers, with most receiving VA disability. That a majority of SCls occurred with headgear and no body armor may reflect inadequate shock absorption in protective equipment not optimized for airborne landings. Low rate of Medevac rescue may suggest tertiary medical care was not attainable or that SCls were deemed minor at time of injury. 


\section{Analysis of Non-Motor Vehicle Accident Etiologies of Spinal Cord Injury in the United States from 1972-} 2018.

Remi A. Kessler BA; John Rutland BA; Raj K. Shrivastava MD; Ansh Bhammar; Constantinos G. Hadjipanayis MD, PhD; Jonathan J. Rasouli MD; Joshua B. Bederson MD; Deborah L. Benzil MD, FACS, FAANS

Introduction: There are $\sim 18,000$ new cases of $\mathrm{SCl}$ annually in the US, with initial hospitalization costs at $\sim \$ 130,000 /$ patient and $>1 / 3$ will be readmitted annually. While MVAs are the US's leading cause of SCI, the non-MVA etiologies are incompletely understood and comprise over $\sim 60 \%$ of all SCl.

Methods: This study used a retrospective cohort-design, including all patients sustaining SCI in the NSCISC database (1972-2018). SCI was defined as occurrence of a lesion of neural elements in the spinal-canal, resulting in deficit. The NSCISC database is the largest, prospective multi-center study capturing data from US SCl patients.

Results: 34,206 SCI patients met inclusion criteria. Violence-related SCI rose from a low of 13\% (1972-1979), peaked at $29 \%$ (1990-1994), and declined to $14 \%$ by $2015-2018$. Sports-related SCls were $14 \%$ in $1972-1979$, and declined to $8 \%$ by $2015-2018$. Notably, SCI from falls originated at $16 \%$ in $1972-1997$ and rose to $32 \%$ by 2015-2018. SCls due to medical/surgical complications were at 1\% in 1972-1979 and rose to 4\% (2015-2018).

Conclusion: Sports-related SCls have a stable downward trend, likely due to awareness of sports-related trauma and adoption of safety equipment/regulations. Incidence from falls and surgical complications has risen, indicating the importance of elderly fall education/prevention. The increase in acquired SCls related to surgical complications may reflect increasing rates of elective spine cases, with spinal fusion procedures increasing by $70 \%$ between 2001 and 2011 . 
Spine Summit Meeting Abstracts, May 2021

8:44 - 8:46 am

\section{Mesenchymal Stem Cells and Curcumin Enhance Spinal Cord Injury Recovery by Modulating the Immune Response}

Anubhav G. Amin MD; Lucia Machova-Urdzikova; Chirag D. Gandhi MD, FACS, FAANS; Meic H. Schmidt MD, MBA; Pavla Jendelova; Meena Jhanwar-Uniyal PhD

Introduction: Traumatic spinal cord injury ( $\mathrm{SCl}$ ) triggers asevere inflammatory reaction which hinders regeneration and recovery. Mesenchymal stem cell (MSC) transplantation is a promising treatment option for $\mathrm{SCI}$ to replace damaged neurons/astrocytes. Also, Curcumin longo, a naturally occurring compound, displays potent anti-inflammatory activity via the NF- pathway.

Methods: Rats underwent a T9-10 laminectomy followed by spinal cord contusion and were randomized to four treatment groups. Rats received MSC or saline intrathecally 7 days after $\mathrm{SCl}$ at the site of injury; while curcumin $(0.5 \mathrm{mmol} / \mathrm{kg}$ or $60 \mathrm{mg} / \mathrm{kg})$ or DMSO was administered daily intraperitoneally. Recovery was measured via multiple functional tests. Secretory inflammatory cytokines, immune gene-expression array, and NF-k $\beta$ activity were evaluated via immunoassays at multiple time points. White and grey matter scarring was measured via immunohistochemistry of tissues proximal, caudal, and at the injury site. Neuronal Regeneration was determined by measuring protoplasmic astrocytes and axonal sprouting.

Results: SCI recovery was significantly better in the MSC + curcumin co-treatment group in comparison to either therapy alone and in comparison to the control group. This improvement was greater than the predicted additive effects of both treatments, suggesting a synergistic effect. MSC + curcumin improved gray and white matter tissue sparing. Cytokine levels displayed a biphasic effect, where IL-6 and IL-12p70 levels increased robustly at post-injury day 28 , while remaining lower in early stages of injury. Alternatively, TNFa was increased day 7 post-injury, but decreased at day 28 in treatment groups. NF-kb activity was suppressed at all measured time points.

Conclusion: These results provide evidence that MSCs plus Curcumin synergistically improve recovery from $\mathrm{SCl}$ by modulating the immunological response via the NF-k $\beta$ pathway, therefore underscoring the potential use of these treatments in humans. 
8:46 - 8:48 am

\section{Variation in Elective Anterior Cervical Discectomy and Fusion Outcomes between Patients with or without Mental IIIness}

Jake Berg; Mohammed A. Alvi MD; Waseem Wahood; Allie J. Canoy Illies; Anshit Goyal MBBS; Panagiotis Kerezoudis; Benjamin D. Elder MD, PhD; Mohamad Bydon MD

Introduction: Patients with a comorbid mental illness have been observed to have worse outcomes following surgery compared to individuals without any mental illness comorbidities. However, not much is known about the effects of mental disorders on patient outcomes following spinal surgery.

Methods: The National Readmissions Database (NRD) was queried for patients undergoing an ACDF between 2012 and September 30th, 2015, the fourth quarter of the year. The presence of anxiety, major depressive disorder, concurrent anxiety and major depressive disorder, and schizophrenia were determined using International Classification of Diseases, Ninth Revision (ICD-9) codes. Multivariable regression was used to establish an association between mental illness and the risk of 30- and 90- day readmission.

Results: A total of 139,877 patients undergoing an elective ACDF from 2012 up to the end of the third quarter of 2015 were identified. Upon multivariable analysis of procedural related readmissions and adjusting for an array of patient and hospital related factors, patients with schizophrenia, compared to controls, had a significantly higher risk of 30-day readmission $(O R=2.62, p=0.002)$, while patients with schizophrenia $(O R=1.92, p=0.016)$ and those with anxiety $(O R=1.13, p=0.023)$ had a significantly higher risk of 90-day readmission.

Conclusion: Our analysis indicates that mental illness comorbidities may be associated with adverse postsurgical outcomes including increased rates of procedure related readmission following elective spinal surgery. 
Spine Summit Meeting Abstracts, May 2021

8:48 - 8:50 am

\section{Prognostic Factors in Degenerative Cervical Myelopathy for Patients Managed Operatively and Non- Operatively}

Allan R. Martin BASc, MD; Sukhvinder Kalsi-Ryan MSc, BScPT; Muhammad Akbar BSc, MD; Jetan H. Badhiwala; Jefferson R. Wilson MD, PhD; Lindsay Tetreault Bsc; Aria Nouri MD, MSc; Anna Rienmuller; Eric M. Massicotte MD; Michael G. Fehlings MD, PhD, FRCS(C), FACS

Introduction: Degenerative cervical myelopathy (DCM) is the most common pathology affecting the spinal cord, but few factors have been identified that predict outcomes with or without surgery.

Methods: Patients with a diagnosis of DCM were enrolled prospectively. In patients managed non-operatively, a binary outcome variable of neurological deterioration was defined based on assessment by the neurosurgeon in clinic. For post-operative recovery, the modified Japanese Orthopedic Association (mJOA) recovery ratio was used as the outcome variable. Baseline data were analyzed for univariate associations with the outcome variables using Chi-squared, T, and Pearson correlation tests. Logistic and linear regression models with backward stepwise elimination were used for multivariate analysis.

Results: In 117 patients, deterioration was more common with lower baseline GRASSP-dexterity (nondominant hand: $p=0.001$; dominant: $p=0.006)$, lower mJOA $(p=0.003)$, clumsy hands $(p=0.008)$, numb hands $(p=0.01)$, unsteady gait $(p=0.01)$, longer follow-up $(p=0.02)$, smoking $(p=0.03)$, depression $(p=0.03)$, and decreased grip strength (dominant: $p=0.04$ ); trends were also seen with higher QuickDASH ( $p=0.06)$, Hoffman sign $(p=0.07)$, obesity $(p=0.09)$, and age $(p=0.10)$. In 71 patients, post-operative recovery was improved with stronger baseline grip strength $(p=0.01$ non-dominant), cord compression at $C 4-5(p=0.01)$ and no compression at $\mathrm{C} 2-3(p=0.02)$; trends were observed with diabetes $(p=0.07)$, no cardiac dysfunction $(p=0.08)$, and younger age $(p=0.10)$. Multivariate analysis found independent predictors of mJOA $(p=0.02)$, follow-up duration ( $p=0.02)$, and smoking $(p=0.04)$ for non-operative deterioration, and grip strength (non-dominant: $p=0.02$ ) and $C 4-5$ compression $(p=0.03)$ for post-operative recovery.

Conclusion: Patients with more severe baseline neurological dysfunction, smoking, depression, and longer follow-up appear more likely to deteriorate without surgery, whereas those with preserved grip strength and C4-5 cord compression have better recovery potential. These variables may be useful to inform surgical decision-making and improve outcomes. 
8:50 - 8:52 am

\section{Spine Injury Transport Times to Level 1 Trauma Facility}

Robert Sterner; Nathaniel P. Brooks MD; Clayton Haldeman MD, MHS

Introduction: Acute spinal cord injury results in loss of neurological function and permanent disability for the patient. Early surgical decompression and stabilization have been shown to improve outcomes, with evidence that "ultra early" ( $<12$ hours after injury) surgery provides the most benefit. In states with large rural areas, like Wisconsin, time from injury to surgery is variable and influenced by multiple factors, including distance from a level I trauma center, mode of transport, and time spent in referring hospitals.

Methods: Prehospital time, EMS transport time, and route of transportation (helicopter vs ambulance) were obtained from the trauma registry database for 175 de-identified patients.

Results: T-test showed that EMS transport time ( $4.3 \mathrm{hrs} \pm 3.7)$ was significantly longer than the time spent at the referring hospital $(3.2 \mathrm{hrs} \pm 3.1)$. Additionally, there was no significant difference in time for the route of EMS transport between helicopter (2.5 hrs \pm 2.5$)$ and ambulance (2.8 hrs \pm 4.6$)$.

Conclusion: Timely triage and transport to a level I trauma center is essential in facilitating early decopression and stabilization of spinal cord injury patients. However, there is wide variability in transport time and time spent at referring hospitals. 
8:52 - 8:54 am

\section{Radiographic Predictors of Mechanical Failure Following Posterior Fusion Surgery in Degenerative Cervical Spine Disease}

Nida Fatima; John H. Shin MD

Introduction: Mechanical failure (MF) in the context of posterior fusion surgery is attributed to the active range of motion and cyclic loading of the cervical spine, this leads to mechanical instability which eventually is the most common etiology of reoperation

Methods: Consecutive patients with DCSD presented at an academic tertiary care hospital from 2009 to 2019 were identified through retrospective chart review. For outcome, univariable association with the postoperative cervical alignment radiographic characteristics were first determined using logistic regression, and a prediction model was then constructed using all characteristics significant at the 0.30 alpha level

Results: Our study cohort included 199 patients (50.8\% females, mean age:65.5 \pm 12.5 years), with a median clinical follow-up of 361 days. The cumulative incidence of MF was $5.0 \%$ in our cohort, with an estimated failure rate of $50 \%$ at the 1-year which increased to $80 \%$ in 3-years following posterior fusion surgery. Multivariable regression analysis revealed that $>5$ number of vertebral levels fused were significant independent predictor of MF by 9.5 -folds (OR:9.56,95\% $\mathrm{Cl}: 1.01-98.3, \mathrm{p}=0.05$ ). Furthermore, immediate postoperative $\mathrm{T} 1$ slope $>25^{\circ}$ was significantly associated with MF by 23.5 folds (OR:23.5,95\% $\mathrm{Cl}: 1.92-$ $287.4, p=0.01$ ). The comparative change in the C2-7 SVA of $>-21 \mathrm{~mm}$ was a significant independent predictor of MF by 13.3 folds (OR:13.3,95\% $\mathrm{Cl}: 1.46-121.7, \mathrm{p}=0.02)$.

Conclusion: Our study corroborates that the fixation range $>5$, post-operative T1 slope $>25 \circ$ and $\Delta \mathrm{C} 2-7$ SVA $\leq-21 \mathrm{~mm}$ are independent predictors of MF following posterior fusion surgery in DCSD. 
8:54 - 8:56 am

\section{Hounsfield Units in the Thoracic Spine Correlate with Bone Mineral Density on Dual Energy X-ray Absorptiometry}

Pierce Peters; Anthony L. Mikula MD; Soliman Oushy MD; Jeremy L. Fogelson MD; Benjamin D. Elder MD, $\mathrm{PhD}$

Introduction: Hounsfield units (HU) of lumbar spine correlate with bone mineral density (BMD) as measured on dual energy x-ray absorptiometry (DXA) and can be used as opportunistic osteoporosis screening. However, HU outside the lumbar spine have not been investigated.

Methods: A retrospective chart review identified patients with a DXA and CT encompassing the thoracic spine within a one year period of time. HU were measured in the thoracic spine at T1-T2 and T11-T12. BMD and TScores in the femoral neck, hip, and lumbar spine were collected.

Results: One-hundred patients were identified for analysis with an average age of 55 years. Thoracic HU Pearson correlation coefficient to lumbar BMD was 0.48 ( $p$-value $<0.0001$ ), to hip BMD was 0.51 ( $p$-value $<0.0001$ ), and to femoral neck BMD was 0.31 ( $p$-value $=0.0024)$. The average thoracic $\mathrm{HU} \pm$ standard deviation for patients with World Health Organization (WHO) classified osteoporosis ( $N=30)$ was $149.9 \pm 54.3$, osteopenia ( $\mathrm{N}=49$ ) was $181.5 \pm 46.3$, and normal bone density $(\mathrm{N}=21)$ was $210.5 \pm 39.4$. Analysis of variance (ANOVA) showed a statistically significant difference in HU between all three groups, most strongly between patients with osteoporosis and normal bone density $(p<0.0001)$.

Conclusion: Thoracic vertebral HU correlated moderately well with lumbar and hip BMD on DXA but poorly with femoral neck BMD. Thoracic HUs differed between patients with osteoporosis, osteopenia, and normal BMD. 
8:56 - 8:58 am

\section{Peri-Operative and Swallowing Outcomes in Patients Undergoing Four and Five Level ACDF Surgery}

Samuel H. Farber MD; David Mauler; Soumya Sagar; Mark A. Pacult ; Corey T. Walker MD; James Zhou MD; Jay D. Turner MD, PhD; Juan S. Uribe MD, FAANS; Steve W. Chang MD; Udaya K. Kakarla MD

Introduction: Anterior cervical discectomy and fusion (ACDF) is one of the most common neurosurgical procedures performed to treat a variety of pathologies in the cervical spine. Literature on ACDF surgery for four or more segments is sparse. Increasing the number of levels increases surgical complexity, tissue retraction, and risks of complications, particularly dysphagia.

Methods: We retrospectively reviewed patients who underwent four or five level ACDF at our institution over a six-year period (May 2013-May 2019). Patient demographics, peri-operative complications, re-admission rates, and swallowing outcomes were recorded. Outcomes were analyzed with a multivariate linear regression.

Results: A total of 177 patients were included ( 4 level $=169,5$ level $=8$ ). The average age was 60.5 years and $53.7 \%$ were males. The majority (87.6\%) underwent surgery at C3-7. $13.6 \%$ underwent a corpectomy. $56.5 \%$ of patients experienced transient dysphagia after surgery. The average length to solid food intake was 2.3 (2.1) days. 5.1\% of patients required treatment with a feeding tube due to severe approach-related dysphagia. Peri-operative complication rates were uniformly low. The average length of stay (LOS) was 5.1 (4.8) days. Corpectomy was positively correlated with increased days to solid food intake $(p=0.004)$ and increased LOS ( $p=0.004$ ). $82.5 \%$ of patients were discharged home. $7.9 \%$ of patients returned to the ED within 30 days and $2.8 \%$ required readmission. $1.1 \%$ required revision surgery within 30 days.

Conclusion: This is the largest series of four and five level ACDF surgery reported to date. We found that this procedure can be performed safely with minimal intra-operative complications. Over half of patients experience transient dysphagia after surgery but the large majority are discharged home without long-term sequelae. Corpectomy was associated with increased time to food intake and increased LOS. 
8:58 - 9:00 am

\section{Early versus Late Surgical Decompression for Central Cord Syndrome: An Analysis of Prospective, Multicenter Data}

Jetan H. Badhiwala MD; Jefferson R. Wilson MD, PhD; Michael G. Fehlings MD, PhD, FRCS(C), FACS

Introduction: The optimal management of central cord syndrome (CCS) remains unclear; yet this pattern of injury is becoming increasingly commonowing to the aging population.

Methods: Patients who underwent surgery for CCS (LEMS-UEMS $\geq 5$ ) were identified from three prospective, multi-center SCI datasets (NACTN; STASCIS; NASCIS III). Propensity scores were calculated for early $(<24$ hrs) versus late ( $\geq 24 \mathrm{hrs}$ ) surgery adjusting for age, injury mechanism, data source, and baseline ASIA motor score (AMS), AIS grade, and neurological level. Propensity score matching was performed in a 1:1 ratio. The primary endpoint was motor recovery (UEMS; LEMS; AMS) at 1 year. Secondary endpoints were FIM motor score and complete independence in each FIM motor domain at 1 year. Effect sizes for outcomes were summarized by mean difference (MD) or odds ratio (OR).

Results: The final cohort had 186 patients. Baseline characteristics were balanced between matched early $(N=93)$ and late $(N=93)$ surgery groups. Early surgery resulted in significantly improved recovery in upper limb (MD 2.3, $\mathrm{P}=0.047$ ), but not lower limb (MD 1.1, $\mathrm{P}=0.256$ ), motor function, as compared with late surgery.

Concerning overall motor function, there was a strong, but non-significant, trend toward improved recovery with early surgery (MD 3.2, $\mathrm{P}=0.065$ ). More patients in the early surgery group appeared to achieve complete independence in various functional activities, particularly those involving upper limb function; however, these associations did not reach statistical significance, and there was no difference in 1-year FIM motor score (MD 4.4, $\mathrm{P}=0.182)$.

Conclusion: In patients with CCS, early surgical decompression (24 hrs) resulted in improved recovery in upper limb motor function at 1 year. Treatment paradigms for central cord syndrome may need to be redefined to encompass early surgical decompression as a neuroprotective therapy. 
9:00 - 9:02 am

Cervical Fractures with associated Vertebral Artery Dissections: an analysis of a 310 patient cohort.

Michael Cloney MD, MPH; Hooman Azad; Nikil Prasad BS; Akash Mitra; Tyler R. Koski MD; Nader S. Dahdaleh MD

Introduction: Cervical fractures can primarily cause severe neurologic injury, and can be associated with secondary processes such as vertebral artery dissections (VADs) with further negative consequences.

Methods: We retrospectively analyzed all 310 patients who were treated at our center for VADs from 20042018. Patient, event, and treatment characteristics were collected, including: demographics, Charlson Comorbidity Index, cause of VAD, location of VAD, complications, medical therapy, and modified Rankin scores (mRS) at various times.

Results: Of the 310 patients with vertebral artery dissections identified, 40 (12.9\%) had associated cervical fractures. Fractures were associated with having violence as the mechanism of injury leading to presentation (OR 16.17 [1.03, 251.74], $p=0.048$ ), and motor vehicle collisions showed a trend toward significance (OR 4.36 $[0.89,21.24], p=0.068)$. Fractures were associated with having a worse $m R S$ at discharge (OR 39.76 [2.53, 624.41), $p=0.009$ ), and a higher likelihood of not being functionally independent at both discharge (OR 10.51 [4.49, 24.61], p0.001) and follow up (OR 5.66 [1.09, 29.54], $p=0.040)$, with a trend toward no improvement or worsening at three months (OR $2.15[1.00,4.65], p=0.051)$. While there was a trend toward a higher proportion of strokes among patients with associated fractures $(p=0.0549)$, fracture was not an independent predictor of a comorbid stroke, and being trauma-associated was a negative predictor of stroke among vertebral artery dissection patients (OR 0.31 [0.14, 0.67], $p=0.004$ )

Conclusion: While vertebral artery dissection is a feared cause of neurologic injury, for patients with cervical fractures, the fracture itself is the primary driver of neurologic outcomes. Cervical fractures do not increase the risk of stroke from a vertebral artery dissection. 
9:02 - 9:04 am

\section{Self-Assembling Peptide Material to Optimize Human Stem Cell-Based Repair of the Traumatically Injured Spinal Cord}

Christopher Saagar Ahuja MD; Mohammad Khazaei ; Maryam Dadabhoy; Nitya Gulati; Niharikaa Aiyar; Vjura Senthilnathan; Nayaab Punjani; Ali Hasan; Inaara Walji; Yao Yao; Michael G. Fehlings MD, PhD, FRCS(C) FACS

Introduction: Human induced pluripotent stem cell-derived neural stem cells (hiPS-NSCs) are a potentially autologous therapeutic approach to regenerate after traumatic spinal cord injury (SCl). Unfortunately, the majority of chronically injured patients develop ex vacuo microcystic cavitations which prevent regenerative cell migration and neurite outgrowth. QL6 $\left(\mathrm{K}_{2}(\mathrm{QL})_{6} \mathrm{~K}_{2}\right.$; Medtronic Inc.) is a novel, pH-neutral, biomaterial which selfassembles into an extracellular matrix (ECM)-like lattice in vivo. It has previously been shown to support endogenous and exogenous mouse cell survival. However, its ability to support human grafts continues to be a critical knowledge gap.

Methods: Nonvirally-generated hiPS-NSCs were cultured on QL6 biomaterial against an ECM control. Adhesion was assessed by EDTA assay and qPCR. hiPS-NSC survival, division, and neurosphere formation were extensively characterized through immunohistochemistry and electron microscopy. T-cell deficient rats capable of supporting a human graft were given a clinically-relevant chronic C6-7 clip-contusion injury or sham surgery(laminectomy alone). Animals were randomized to: (1)vehicle, (2)hiPS-NSCs, (3)QL6, (4)QL6+hiPSNSCs. All rats underwent delayed daily treadmill rehabilitation. A subset of animals underwent high throughput single-cell RNA sequencing (scRNAseq).

Results: hiPS-NSCs proliferated robustly on the QL6 biomaterial (Ki67 ${ }^{+} / \mathrm{DAPI}{ }^{+} ; 29 \% \mathrm{vs} 6 \%$; $\left.\mathrm{p}<0.01\right)$. An EDTA adhesion assay demonstrated that human NSC binding to QL6 is largely driven by calcium-independent mechanisms. Importantly, QL6 enhanced the formation of adherent neurospheres, the native conformation of NSCs. EM-imaging provided the first images of an interwoven human NSC-QL6 biomaterial interaction. A battery of blinded sensorimotor assessments are ongoing with a 22-week post-injury endpoint. However, scRNAseq differential gene expression analyses suggest the QL6 is altering the fate determination of the human cell graft after transplantation.

Conclusion: This work provides key proof-of-concept data that QL6 self-assembling peptide can support translationally-relevant human iPS-NSCs for use in traumatic SCI. 
9:04 - 9:06 am

\section{The Utility of Preoperative Laboratory Testing in Assessing Risk of Adverse Outcomes Following ACDF: Insights from NSQIP}

Mohamad Bydon MD; Jad Zreik BS; Anshit Goyal MBBS; Mohammed A. Alvi MD; Brett Freedman

Introduction: Preoperative laboratory tests are varied in their administration among physicians, and previous studies have published conflicting reports on their utility. Anterior cervical discectomy and fusion (ACDF) is a commonly performed spine surgery, and patients often undergo preoperative testing.

Methods: ACS-NSQIP was queried from 2012-2017 to identify patients undergoing elective ACDF. Multivariable logistic regression was performed to assess the association between abnormal laboratory test values and adverse 30-day outcomes. Relative predictor importance was determined using an importance metric defined as Wald $x^{2}$ penalized by degrees of freedom.

Results: A total of 47,111 patients were included. On multivariable analysis, high creatinine $(p=0.030)$, anemia $(p<0.001)$, hyponatremia $(p=0.034)$, and leukocytosis $(p<0.001)$ were found to be significantly associated with any 30-day complications. Anemia $(p<0.001)$, hypernatremia $(p=0.028)$, hyponatremia $(p=0.016)$, and leukocytosis $(p<0.001)$ were found to be significantly associated with serious 30 -day complications. High creatinine $(p=0.027)$, anemia $(p<0.001)$, hyponatremia $(p=0.047)$, and leukocytosis $(p=0.004)$ were found to be significantly associated with 30-day unplanned readmissions. High blood urea nitrogen $(p=0.007)$, high creatinine $(p=0.028)$, anemia $(p<0.001)$, low platelet count $(p<0.001)$, hyponatremia $(p<0.001)$, and leukocytosis $(p<0.001)$ were found to be significantly associated with non-home discharge. Predictor importance analysis revealed that abnormal preoperative lab values were an important determinant in predicting these 30-day outcomes.

Conclusion: Our analyses indicate that abnormal preoperative lab values are associated with increased risk for adverse outcomes following elective ACDF and can be used in predictive analyses of outcomes. 
9:06 - 9:08 am

\section{Nutritional Status as a 30-d Predictor of Complications and Mortality Following Posterior Cervical Fusion}

Nida Fatima; John H. Shin MD

Introduction: Preoperative serum albumin serves as a clinical marker of nutritional status. There exists a paucity of literature in the realm of postoperative outcomes following posterior cervical fusion (PCF) in patients who are nutritionally depleted.

Methods: Patients who underwent PCF from the American College of Surgeons, National Surgical Quality Improvement Program between 2005 and 2017 were extracted. Multivariable logistic regression analysis (MLR) model was used to predict the short-term complications and mortality in patients who had a nutritional deficiency (serum albumin $<3.5 \mathrm{~g} / \mathrm{dl}$ ). All associations were computed as odds ratio $(\mathrm{OR})$ with $95 \%$ confidence interval $(\mathrm{Cl})$.

Results: Statistical analysis included 3, 210 patients (48.8\% females, mean age: $63.9 \pm 13.5$ years) with preoperative serum albumin $<3.5 \mathrm{~g} / \mathrm{dl}$ in $9.3 \%(n=298)$ of the patients. Nutritionally depleted individuals had 3.76-folds independent risk of developing any 30-d complications (OR: $2.76,95 \% \mathrm{Cl}: 2.45-5.78$, $\mathrm{p}<0.001)$. Furthermore, patients with nutritional deficiency were associated with increased risk of postoperative number of red blood cell transfusions (OR: 2.97, 95\% Cl: 2.01-4.39, p<0.001), sepsis (OR: 2.98, $95 \% \mathrm{Cl}: 1.39-6.42, \mathrm{p}=0.005)$, total length of hospital stay (OR: $1.04,95 \% \mathrm{Cl}: 1.02-1.06, \mathrm{p}<0.001$ ), and mortality (OR: $3.63,95 \% \mathrm{Cl}: 1.25-10.4, \mathrm{p}=0.02$ ).

Conclusion: Since preoperative serum albumin is a modifiable risk factor, thus can potentially improve the surgical outcomes following PCF. Our study corroborates that nutritional status is an independent risk factor for complications, length of hospital stay and mortality in patients undergoing PCF 
9:08 - 9:10 am

\section{Timing of Surgical Decompression for Acute Traumatic Spinal Cord Injury: A Study of North American Trauma Centers}

Jetan H. Badhiwala MD; Gerald Lebovic; Jefferson R. Wilson MD, PhD; Michael G. Fehlings MD, PhD, FRCS(C), FACS; Christopher D. Witiw MD

Introduction: In recent years, there has been growing recognition of the role of early decompressive surgery in patients with acute traumatic spinal cord injury $(\mathrm{SCl})$. Despite this, there are limited data pertaining to current practices in North America.

Methods: Adults with acute SCI who underwent decompressive surgery within 7 days of injury were identified from the ACS TQIP database for 2010-2016. Mixed-effects modeling with a random intercept for trauma center was used to examine the adjusted association of patient- and hospital-level variables with time to decompression. The random-effects output of the model was used to assess the risk-adjusted variability in timeliness of surgery across trauma centers. Outlier hospitals were identified and the intraclass correlation coefficient (ICC) was calculated.

Results: A total of 15,388 patients admitted to 438 TQIP centers were eligible (mean age, 46.2 yrs). Older age, black race, non-private insurance, greater comorbid status, fall mechanism, cervical incomplete injury, thoracic complete injury, lower GCS score, and presence of concomitant head, facial, abdominal, or lower extremity injury were associated with longer time to decompressive surgery. Time to decompression decreased incrementally from 2010 to 2016 and was inversely correlated with hospital volume of SCl patients treated. Seventy-nine hospitals were outliers, with a significantly shorter or longer time to decompression than average. The ICC was $8.8 \%$.

Conclusion: The timing of surgical decompression for acute traumatic $\mathrm{SCl}$ is influenced to some degree by patient- and hospital-related factors. However, there remains substantial variability between trauma centers in timeliness of surgery that that is not explained by differences in case-mix or hospital characteristics. 
9:10 - 9:12 am

\title{
Quantitative Romberg using a Force Plate: An Objective Measure for Cervical Myelopathy
}

\author{
Jeffrey L. Gum M.D.; Steven D. Glassman; Morgan Brown; Christy Daniels; Joseph Laratta; Leah Carreon
}

Introduction: Surgical decision making for Cervical Spondylotic Myelopathy (CSM) relies on symptoms and physical examination. The Romberg test is a clinical exam used to identify balance issues with CSM, but has subjective interpretation and has a binary (positive or negative) result. Using a force plate, the area, frequency and speed of sway with eyes open and eyes closed can be quantified. This objective measurement has the potential for earlier intervention and/or to monitor treatment progress. Additionally, this measurement tool is more practical than a formal gait analysis in the clinical setting.

Methods: Patients with CSM requiring surgery and healthy normal volunteers were asked to perform the Romberg test while on a force plate measuring the center of pressure: standing up straight with arms extended for 30 seconds with eyes open, followed by 30 seconds with eyes closed. The change in total sway area (TSA), sway frequency and sway speed with eyes closed and eyes open were calculated.

Results: 27 of 48 CSM patients were age-matched to 27 healthy volunteers with a mean age of 54 years. The change in TSA $\left(211.5 \mathrm{~mm}^{2}\right)$, frequency $(365.4 / \mathrm{s})$ and speed $(7.0 \mathrm{~m} / \mathrm{s})$ was statistically greater in the CSM group compared to normal TSA $\left(87.8 \mathrm{~mm}^{2}, \mathrm{p}=0.005\right)$, frequency $(62.1 / \mathrm{s}, \mathrm{p}=0.008)$ and speed $(4.2 \mathrm{~m} / \mathrm{s}, \mathrm{p}=0.085)$.

Conclusion: Poor standing balance can be quantified in patients with CSM. The Romberg test on a force plate may help diagnose and evaluate CSM. Further studies are needed to determine its utility to measure treatment effectiveness. 
9:12 - 9:14 am

\section{Anterior Discectomy and Fusion (ACDF): Does the Teaching Status of the Hospital Matter?}

Aya Akhras MBBS; Waseem Wahood MS; Mohammed A. Alvi MD; Anshit Goyal MBBS; Yagiz U. Yolcu MD; Mohamad Bydon MD

Introduction: Teaching hospitals are responsible for the education of residents, and the base for research and advancement in an era of evidence based medicine. There is still scarce research in the field of neurosurgical outcomes between these institutions, and even less in cervical spine surgery. ACDF is a commonly performed procedure to treat cervical degenerative disc disease refractory to conservative therapy. This rationalizes the investigation of differences in surgical outcomes and mortality between teaching and non-teaching hospitals.

Methods: The Nationwide Inpatient Sample (NIS) was queried between 2012 and 2015 to identify patients with cervical degenerative disc disease (DDD) undergoing ACDF. Patients with concurrent lumbar DDD, spinal trauma, infections, or tumors were excluded. Multivariable regression was conducted to assess the association of teaching status of urban hospitals with outcomes including: mortality, cost, and length of stay. Results are represented with odds ratios (ORs) for categorical outcomes, and coefficients for continuous outcomes.

Results: A total of 83,878 patients were identified in NIS with 32,108 (38.3\%) in non-teaching hospitals and $51,770(61.7 \%)$ in teaching hospitals. Mean age (SD) was 55.4 (11.6) and 55.1 (11.6) years, respectively. Adjusting for comorbidities and other patient and hospital factors, teaching status had no effect on mortality (OR: $0.77,95 \% \mathrm{Cl}$ : 0.43 to $1.39, \mathrm{p}=0.39$ ) or cost (coef.:-112.6, 95\% Cl: -248.9 to 23.6, $\mathrm{p}=0.11$ ). However, patients at teaching hospitals had longer stays, on average (coef.: $0.16,95 \% \mathrm{Cl}: 0.14$ to $0.19, p<0.001$ ).

Conclusion: Results of this study will be crucial in influencing the choice of care for patients regarding this common procedure, in order to optimize and better facilitate their healthcare experience. 
9:14 - 9:16 am

\section{Local Retropharyngeal Space Anesthetic for Dysphagia Reduction After Anterior Cervical Discectomy and Fusion Surgery}

Alan T. Villavicencio MD; Ewell L. Nelson MD; Sharad Rajpal MD; Kara D. Beasley DO, MBe; Vinod Kantha

BA; Sigita Burneikiene MD

Introduction: The main objective of this study was to analyze the ability of local anesthetic instillation into the retropharyngeal space to reduce dysphagia symptoms and occurrence rates in patients undergoing anterior cervical discectomy and fusion (ACDF) procedures.

Methods: A single-center, prospective, randomized, double-blinded, and placebo-controlled clinical study was performed. We enrolled patients undergoing one- or two-level ACDF procedures for cervical degenerative disc disease with disc herniation, radiculopathy and/or myelopathy symptoms. The patients were randomly assigned (1:1 ratio) to receive either $0.5 \%$ bupivacaine hydrochloride or $0.9 \% \mathrm{NaCl}$ solution.

Results: In total, 120 patients were randomized and 111 completed the study. Twenty-seven (23.7\%) patients reported no dysphagia symptoms: $15(25.9 \%)$ in the investigational and $12(22.6 \%)$ in the control groups. There were no statistically significant differences in duration of dysphagia symptoms, Swallowing-Quality of Life (SWAL-QOL) survey or pain scores between the investigational or control patient groups at any of the follow-up time points. Controlling for independent variables, only younger age significantly predicted dysphagia symptoms at discharge, 2-week, and 3-month follow-ups ( $p \leq 0.03 ; \mathrm{R} \geq-0.038$; OR=0.96, 95\% $\mathrm{Cl} 0.93-0.99$. Female gender was associated with lower SWAL-QOL scores at discharge $(p=0.046 ; R=0.87 ; O R=2.38,95 \%$ Cl 1.02-5.56).

A total of $8(13.8 \%)$ and $6(11.3 \%)$ patients in the investigational and control groups, respectively, were referred to a specialist or underwent speech therapy for their dysphagia symptoms. There were no adverse reactions to the study drug observed.

Conclusion: Local retropharyngeal space anesthetic instillation did not reduce dysphagia symptoms or occurrence rates in patients undergoing anterior discectomy and fusion surgeries. 
9:16 - 9:18 am

\title{
Clinical and Radiographic Benefits of Skipping C7 Instrumentation in Posterior Cervicothoracic Fusion
}

\author{
Saavan Patel; Matthew K. Tobin BS; Cristian Gragnaniello; Sergey Neckrysh MD, FAANS
}

Introduction: Including C7 level as a point of fixation during a posterior cervicothoracic fusion can be challenging because it requires additional work of either placing side connectors to a single rod or placing two rods. Our clinical observations suggested that skipping instrumentation at C7 in a multiple level posterior cervicothoracic fusion will result in minimal intraoperative complications, decreased surgical time, while still maintaining fixation, sagittal balance parameters and successful rates of fusion.

Methods: This is a retrospective chart review of 93 consecutive patients who underwent multilevel PCF at our Institution. Out of 93 patients, 8 patients were instrumented at C7, serving as the control group, while the remaining patients were not. Evaluation of efficacy was based on intraoperative complications, operative time, estimated blood loss, significant long-term complications, and radiographic evidence of fusion.

Results: Skipping the C7 level resulted in a reduction in estimated blood loss $(669 \pm 769 \mathrm{~mL}$ vs $1369 \pm 1591 \mathrm{~mL})$ and decreased operative time (207 \pm 116 minutes vs $218 \pm 85$ minutes). Complications were minimal in both group and not statistically significant. Additionally, patients skipped at C7 maintained sagittal balance with fusion rates similar to control patients at follow-up. Furthermore, $\mathrm{C} 7$ bridge patients had a significantly increased postoperative SVA $\left(35.8^{\circ}\right.$ vs $\left.22.6^{\circ}, p=0.015\right)$ with significantly increased SVA correction $\left(10.9^{\circ}\right.$ vs $\left.15.6^{\circ}, p<0.0001\right)$. No significant long-term complications in either group.

Conclusion: Skipping instrumentation at C7 in a multilevel posterior cervicothoracic fusion demonstrates lower EBL and faster operative times compared to performing instrumentation at the $\mathrm{C} 7$ level. Additionally, postoperative assessment revealed identical rates of fusion between groups. The results of this study show the clinical benefits of skipping $\mathrm{C} 7$ to minimize surgical risk and complications in patients undergoing posterior cervical fusion across the cervicothoracic junction. 
9:18 - 9:20 am

\section{ASA Class Surpasses the Diagnosis-Related Group System in Predicting Costs of Posterior Cervical Discectomy and Fusion}

Annie E. Arrighi-Allisan BA; Sean N. Neifert BS; Rebecca Baron; Frank Yuk MD; Jeffrey Gilligan MD; John M. Caridi MD

Introduction: Posterior cervical discectomy and fusion (PCDF) is designed to treat numerous degenerative pathologies of the cervical spine. Medicare Reimbursement for PCDF is tied to Diagnosis-Related Groups (DRGs); however, numerous other classification systems have been utilized in the surgical literature to stratify patients by comorbidities and operative risk. The superiority of DRG to other classification systems in predicting PCDF patient costs remains unproven.

Methods: Patients undergoing PCDF at a single institution from 2008 to 2016 were queried; those undergoing simultaneous ACDF were excluded. A tiered system was created that accounted for the increasing levels of comorbidity burden reflected by the DRG, $\mathrm{ECI}, \mathrm{CCl}$ and $\mathrm{ASA}$ Class for each patient. Each system was correlated to demographic factors, and predictive models for cost were created and compared among the several methodologies.

Results: The mean age of PCDF patients increased commensurately as DRG, ASA Class, and ECI level increased $(P<0.0001)$. ASA Class yielded the most accurate cost prediction for PCDF patients $(\$ 4,426 ; 95 \% \mathrm{Cl}$ $\left.\$ 1,818-\$ 5,476 ; P<0.0001 ; R^{2}=0.06\right)$. DRG and $\mathrm{CCl}$ exhibited equally predictive cost estimates to one another $\left(\$ 2,767 ; \$ 1,818-\$ 3,716 ; P<0.0001 ; R^{2}=0.03\right.$ and $\$ 2,342 ; \$ 1,500-\$ 2,185 ; P<0.0001 ; R^{2}=0.03$, respectively). The $\mathrm{ECl}$ was the least predictive system of the four $(\$ 1,307 ; 95 \% \mathrm{Cl} \$ 827-\$ 1,788 ; P<0.0001$; $\left.\mathrm{R}^{2}=0.02\right)$.

Conclusion: ASA Class is most predictive of episode-based costs for PCDF, challenging the utility of the DRG classification for Medicare reimbursement. Further studies are needed to elucidate whether ASA Class remains the most accurate predictor of costs for additional spine surgeries. 
9:20 - 9:22 am

\section{Anterior Hybrid Fusion For Degenerative Cervical Myelopathy: Case Series, Systematic Review, and Meta-analysis}

Andrew Platt MD; W. H. Andrew Ryu BSc, MSc, MTM, MD; Harel Deutsch MD

Introduction: Degenerative cervical myelopathy, with an annual incidence of 41 per million in North America, is among the most common indications for cervical spine surgery.

Methods: We performed a retrospective case series of patients undergoing Hybrid fusion and analyzed demographics, blood loss, complications, patient reported outcomes and C2-7 cervical lordosis. We performed a systematic review and meta-analysis to assess superiority in terms of operative time, blood loss, cervical lordosis, JOA/NDI, fusion, and complications.

Results: When comparing Hybrid fusion to ACCF there was a statistically significant increase in postoperative cervical lordosis in the hybrid group. We found that patients undergoing three level ACDF had significantly less blood loss than patients undergoing hybrid fusion who in turn had significantly less blood loss than patients undergoing two level corpectomy. Regarding Hybrid fusion and ACCF our meta-analysis showed that Hybrid fusion had a significantly increased fusion rate and a significantly decreased frequency of complications.

Conclusion: We have shown that in comparison to 3 level ACDF, Hybrid fusion has a similar outcome, complication and fusion success rate, however, that in comparison to 2 level corpectomy Hybrid fusion has improved radiographic outcomes, a higher fusion rate, and lower complication rate including lower implant failure/mesh subsidence rate. We recommend hybrid fusion for patients with post-vertebral degenerative cervical myelopathy and 3 level degenerative cervical myelopathy. 
9:22 - 9:24 am

\section{Cost Analysis of Three Surgical Approaches for Cervical Spondylotic Myelopathy}

Zoher Ghogawala MD, FACS; Aniksha Balamurugan; Melissa Dunbar; Jenna Whooley BS; Subu N. Magge MD; Robert G. Whitmore MD

Introduction: Cervical Spondylotic Myelopathy (CSM) is the most common cause of spinal cord dysfunction in the world. There is significant practice variation among the surgical options for treatment with varying outcomes and costs. It is estimated that hospital costs for surigcal treatment of CSM exceed 2 billion dollars/ year in the US.

Methods: Coding and billing data were collected from 15 American centers in the randomized CSM-S trial (NCT02076113). Medicare reimbursement was calculated using ICD-9/10 CM, PCS, and CPT codes. Medicare DRG reimbursement was calculated for each surgical approach and for each hospital. DRG and CPT reimbursements were combined to reach a total reimbursement for each case. One-way ANOVA analysis was then performed.

Results: 108 patients had coding and billing data available ( $n=43$ for VF, $n=45$ for DF, and $n=20$ for L). Average total reimbursement for each surgical approach from 2014-2017 was $\$ 19,016$ for VF, $\$ 18,938$ for $\mathrm{DF}$, and $\$ 10,081$ for $\mathrm{L}$. Laminoplasty was significantly less costly than either dorsal or ventral fusion $(F=141.37, P<0.001)$.

Conclusion: Cervical laminoplasty was less costly compared to ventral or dorsal fusion for treating CSM within the context of an RCT. Next steps would include calculation of QALYs gained in order to estimate costeffectiveness of these procedures. In addition, further studies would measure the comparative outpatient costs associated with these operations including lost productivity. 
9:24 - 9:26 am

\section{Prevalence and Impact of Anemia on Neurological Status in Patients with Degenerative Cervical Myelopathy}

Aria Nouri MD, MSc; Abhijith V. Matur; Zach Pennington BS; Nora C. Elson BS, MS; Sakibul Huq; A. Karim Ahmed BS; Kishan Patel; William J. Jeong MD; Rani Nasser MD; Enrico Tessitore; Daniel M. Sciubba MD; Joseph S. Cheng MD, MS

Introduction: Degenerative cervical myelopathy (DCM) and radiculopathy are commonly present in older patients, many of whom are also afflicted by anemia due to nutritional deficiencies. Herein, we assess the prevalence and association of anemia with neurological status in patients receiving surgical treatment for DCM or radiculopathy.

Methods: Data including CBC were collected, and Nurick grading performed. Established criteria for diagnosing abnormalities were used to identify microcytosis, macrocytosis, anemia, microcytic anemia, macrocytic anemia, and iron deficiency. Mean Nurick grades were compared between patients with and without lab abnormalities. Multivariable linear regression was used to determine the impact of abnormal conditions on Nurick grades.

Results: 725 patients $(57.1 \pm 11.7$ years) with myelopathy $(n=398)$ and radiculopathy $(n=327)$ were evaluated. Mean preoperative Nurick grade was $2.09 \pm 1.29$, and $2.98 \pm 1.12$ for myelopathy patients. Patients with radiculopathy were significantly younger $(53.8 \pm 11.0 \mathrm{vs} 59.8 \pm 11.6, \mathrm{p}<0.001)$. Anemia was observed in $26.1 \%$ overall, but was more common in myelopathy $(33.7 \% \mathrm{vs} 16.8 \%, \mathrm{p}<0.0001)$. Anemic patients were significantly older ( $p<0.0001)$, less likely current smokers $(20.1 \%$ vs $34.3 \%, p<0.001)$, and more likely to have undergone posterior/combined surgical approaches $(p<0.0001)$. Nurick grading was significantly higher in myelopathy patients with anemia $(3.13 \pm 1.19$ vs $2.91 \pm 1.07, p=0.05)$ and macrocytic anemia $(4.00 \pm 1.41$ vs $2.97 \pm 1.11$, $p=0.04)$. Multivariate regression demonstrated that anemia $(p<0.001)$, age $(p<0.0001)$, and posterior surgical approach $(p<0.0001)$ were related with a worse preoperative Nurick grade.

Conclusion: Prevalence of anemia in DCM and radiculopathy is high, and smoking may mask the presence of anemia. Anemia, and macrocytic anemia specifically, was a predictor of worse neurological status in myelopathy. The presence of anemia may also impact surgical recovery. The reason for the high prevalence of anemia in DCM needs to be further evaluated, and patients with macrocytic anemiashould be evaluated for potential B12 deficiency and may benefit from supplementation. 
9:26 - 9:28 am

\section{Diagnosis-Related Group Classification Best Predicts Episode-Based Costs for Anterior Cervical Discectomy and Fusion}

Annie E. Arrighi-Allisan BA; Sean N. Neifert BS; Rebecca Baron; John M. Caridi MD; Frank Yuk MD; Jeffrey Gilligan MD; Jonathan Gal

Introduction: Anterior cervical discectomy and fusion (ACDF) is performed to treat cervical disc herniation and degenerative disease. Though there exist numerous classification systems that stratify operative risk based on comorbidities and demographic factors, Medicare reimbursement is determined by a patients assigned Diagnosis-Related Group (DRG), a classification system developed in the early 1980s based on clinical characteristics and costs. It remains unclear, however, whether the DRG system still prevails as the most accurate predictor of episode-based costs.

Methods: Patients undergoing ACDF at a single institution from 2008-2016 were reviewed; those undergoing concurrent PCDF were excluded. A tiered system was created that accounted for the increasing levels of comorbidity burden reflected by the DRG, ECI, $\mathrm{CCl}$ and ASA Class for each patient. Each system was correlated to demographic factors, and predictive cost models were compared among the various systems.

Results: The mean age of patients undergoing ACDF increased as DRG level grew; a similar relationship was observed as ASA and ECI increased ( $P<0.0001$ for all). The DRG system provided the best cost estimate for patients undergoing ACDF $\left(\$ 4,162 ; 95 \% \mathrm{Cl} \$ 3,681-\$ 4,645 ; \mathrm{P}<0.0001, \mathrm{R}^{2}=0.1\right)$. ECI and ASA exhibited the second and third most accurate cost predictions, respectively $(\$ 1,270 ; 95 \% \mathrm{Cl} \$ 1,063-\$ 1,477 ; \mathrm{P}<0.0001$; $\mathrm{R}^{2}=0.06$ and $\left.\$ 2,316 ; 95 \% \mathrm{Cl} \$ 1,888-\$ 2,743 ; \mathrm{P}<0.0001 ; \mathrm{R}^{2}=0.04\right)$. The $\mathrm{CCl}$ yielded the least accurate cost prediction of the four $\left(\$ 1,531 ; 95 \% \mathrm{Cl} \$ 1,134-\$ 1,929 ; \mathrm{P}<0.0001 ; \mathrm{R}^{2}=0.02\right)$.

Conclusion: The DRG classification system is superior to ASA Class, $\mathrm{ECl}$, and $\mathrm{CCl}$ when predicting episodebased costs for ACDF. These findings support the continued use of the DRG system when determining ACDF Medicare reimbursement, though future studies are required to explore whether this extends to other surgical disciplines. 
9:28 - 9:30 am

\section{Frailty Predicts Complications Better than Age after Surgery for Cervical Myelopathy: A NSQIP Database Study}

Jamie RF Wilson BA.BM.BCh(OXON).FRCS(Neurosurgery); Jetan H. Badhiwala; Robert Ravinksy; Fan Jiang MD; Michael G. Fehlings MD, PhD, FRCS(C), FACS

Introduction: A direct comparison between assessments of frailty and older age in relation to peri-operative complications for surgery in Degenerative Cervical Myelopathy (DCM) has not been performed.

Methods: Patients who underwent surgery for DCM from the NSQIP database were included. The 5-point modified Frailty Index $(\mathrm{mFI})$ was applied, with descriptive statistics calculated for continuous and categorical variables of demographic and complication metrics. The independent effect of age and frailty on outcomes was evaluated by multivariable regression. For each outcome, a logistic or linear regression model was constructed that included both variables and adjusted for sex, type of fusion, and number of levels as covariates. Effect sizes were summarized by odds ratio (OR) (dichotomous outcomes) or mean difference (MD) (continuous outcomes) and associated $95 \%$ confidence intervals (Cls). To weigh the relative importance of age versus frailty in predicting each outcome, standardized regression coefficients were calculated and their magnitudes directly compared.

Results: 5107 patients (2248 females) were extracted for analysis (mean 71.7 years), with 3298 (64.6\%) undergoing anterior surgery, 1649 (32.3\%) posterior surgery, and $160(3.1 \%)$ combined. The mean mFI was 0.23 (SD 0.16). In the regression analyses, age and $\mathrm{mFI}$ were found to be statistically significant predictors for 30-day mortality, readmission, reoperation, major complication, hospital LOS and discharge home. However, $\mathrm{mFI}$ was found to have a greater effect size than age, as measured by the standardized Beta coefficient, for mortality, reoperation, major complication and discharge home.

Conclusion: Frailty, as measured by the $\mathrm{mFI}$, is a better predictor than age for mortality, reoperation, major complication and discharge home in the first 30 days after surgical treatment for DCM. We recommend this index should be used in preference over age when considering the risks of surgical management in DCM. 
Sunday, March 8, 2020

General Spine Surgery 2/Tumor/Opioids

Abstract Breakout Session

7:30 - 7:32 am

Vertebral Hemangiomas: Diagnosis and Management. A Single Center Experience

Nahom T. Teferi MD

Introduction: Vertebral hemangiomas $(\mathrm{VH})$ are common benign lesions involving the spine. They are often discovered incidentally during evaluation for spinal pain.

Methods: Between 2005 and 2019, 11 cases of VH were managed by our department. There were 2 cervical cases, with the remaining 9 in the thoracic spine. Ten cases were symptomatic, and 1 incidental. Three patients presented with localized pain, and the remaining 7 had neurological deficit consisting of numbness or paraparesis. Decompression with maximal resection of the hemangioma was undertaken in 10 cases, and vertebroplasty in 1.

Results: In the 10 patients who underwent surgery, the preoperative diagnosis of VH was confirmed to be accurate in all but 1 case. There were 2 cervical cases treated with corpectomy. One patient was treated with vertebroplasty, and the remaining 8 patients were treated with maximal decompression. Radiation was used in 2 cases, 1 for recurrence and for residual tumor in the second. Of the 10 patients undergoing decompression, 7 patients had improvement of the neurologic deficit, with resolution of pain in the remaining 3 . None of our cases demonstrated deterioration.

Conclusion: VH are often discovered incidentally during evaluation of patients with back or neck pain. Observation for the asymptomatic lesion is appropriate. For $\mathrm{VH}$ presenting with pain, vertebroplasty has been demonstrated to be effective. For VH presenting with deficit or pain unresponsive to vertebroplasty, decompressive surgery is recommended. Radiation is appropriate in cases of recurrent $\mathrm{VH}$. 
7:32 - 7:34 am

\section{Can We Make Them Walk Again? Predictors of Ambulatory Function in Metastatic Spinal Cord Compression}

Nida Fatima; John H. Shin MD

Introduction: Metastatic spinal cord compression (MSCC) is an oncological emergency, and potentially leads to permanent neurological deficits. As such, the optimum predictors of ambulatory status after decompressive surgery (DS) in MSCC are poorly delineated.

Methods: Consecutive patients who underwent DS for MSCC at an academic tertiary care hospital from 20112018 were identified through retrospective chart review of a prospectively maintained database. Multivariate Cox proportional hazards regression model was used to identify the predictors of ambulatory status after DS in MSCC at the last follow-up examination.

Results: With a median age of 67 years (range, 33-84 years), there were 75 male patients (63\%) in our cohort. The median clinical follow-up of patients was 345 days (range,90-678 days). From the multivariate analysis, presence of preoperative ambulation (HR:2.61,95\% Cl:1.46-4.65; $p=0.001)$ independently increased the likelihood of regaining ambulation at the last follow-up evaluation by 2.6 folds. Preoperative radiation therapy (HR: $0.42,95 \% \mathrm{Cl}: 0.25-0.70 ; p=0.001$ ) independently decreased the likelihood of regaining ambulation at the last follow-up evaluation by $68 \%$. For patients with primary genitourinary tumors (HR:0.24,95\% $\mathrm{Cl}: 0.07$ $0.82 ; p=0.02)$, and those with extraspinal metastasis $(>1 / 0)(\mathrm{HR}: 0.57,95 \% \mathrm{Cl}: 0.36-0.92 ; p=0.02)$ had less likelihood of preserving ambulation at the last follow-up examination..

Conclusion: The preoperative ambulatory status and radiation therapy guides regarding the return of ambulatory function, whereas, the presence of extraspinal metastasis and primary tumor histology provides insight for the maintenance of the ambulatory status in patients surviving after DS for MSCC at the last followup. 


\section{7:34 - 7:36 am}

\section{Impact of Dialysis on 30-day Outcomes Following Spinal Fusion Surgery for Pathological Fractures: Insights from NSQIP}

Mohammed A. Alvi MD; Jad Zreik BS; Waseem Wahood; Anshit Goyal MBBS; Brett Freedman; Mohamad Bydon MD

Introduction: Patients with chronic renal failure (CRF) undergoing hemodialysis have been shown to have poor overall health, osteoporosis and altered bone metabolism. However, the impact of hemodialysis on patient outcomes after spinal fusion remains unknown

Methods: We queried the National Surgical Quality Improvement Program (NSQIP) database from 2009-2016 to identify subjects receiving cervical or lumbar fusion for degenerative spine disease. Three-to-one propensityscore matching using sex, age, BMI, and number of operated levels was employed to match patients not undergoing dialysis with those undergoing dialysis. Multivariable conditional regression was used to identify the association between dialysis and 30-day clinical outcomes, after adjusting for confounders

Results: A total of 48,492 patients undergoing cervical fusion were identified; $156(0.32 \%)$ of these were on dialysis. On multivariable regression, dialysis-dependency was found to be associated with increased operative time (Coef. 15.93, 95\% Cl 0.4-31.5, p-value 0.045), length of stay (LOS) (Coef. 6.06, 95\%Cl 4.64-7.48, p-value: $<0.001$ ), 30-day readmissions (OR 1.07, 95\% Cl 1.02-1.12, p-value 0.009), any complications $(\mathrm{OR} 1.08,95 \% \mathrm{Cl}$ 1.03-1.13, p-value 0.002), and serious complications (OR 1.08, 95\% Cl 1.02-1.14, p-value 0.012 ). A total of 25,417 patients undergoing lumbar fusion were identified; $51(0.2 \%)$ of these were on dialysis. On multivariable-regression, dialysis-dependency was found to be associated with significantly higher LOS (Coef. 2.98, 95\% Cl 1.28-4.68, p-value <0.001)

Conclusion: Our analyses indicated that dialysis-dependency is associated with poor peri- and post-operative outcomes following cervical/lumbar fusion for pathological compression fractures 
7:36 - 7:38 am

\section{Predictors of Survival and Scoring System after Decompressive Surgery in Metastatic Spinal Cord Compression}

Nida Fatima; John H. Shin MD

Introduction: The optimal selection of patients who might benefit from decompressive surgery (DS) in metastatic spinal cord compression (MSCC). is poorly defined

Methods: Consecutive patients who underwent DS for MSCC at an academic tertiary care hospital from 20112018 were identified through retrospective chart review of a prospectively maintained database. Multivariate Cox proportional hazards regression model was used to identify the predictors of survival after DS in MSCC at 3-months.

Results: The study cohort included 119 patients (63\% males, median age: 67 years) with a median survival time of 345 days [95\% Confidence Interval $(\mathrm{Cl}): 91.77-652.8$ days). From the multivariate analysis, extraspinal metastasis (31/0) [Hazards Ratio (HR): $2.44,95 \% \mathrm{Cl}: 1.32-4.54 ; \mathrm{p}=0.005$ ] independently increased the risk of mortality by 2.4 folds at 3-months of surgery. Poor modified Frankel grade at presentation (A-B-C/D-E) (HR: 2.17, $95 \% \mathrm{Cl}: 1.13-4.19 ; \mathrm{p}=0.02$ ) independently increased the risk of death at 3-months of $\mathrm{DS}$ by 2.1 folds. For patients with primary genitourinary tumors (HR: $0.15,95 \% \mathrm{Cl}: 0.04-0.52, p=0.003)$, gastrointestinal tumors (HR: $0.32,95 \% \mathrm{Cl}: 0.11-0.92 ; \mathrm{p}=0.03)$, and lung cancer ( $\mathrm{HR}: 0.33,95 \% \mathrm{Cl}: 0.12-0.84 ; \mathrm{p}=0.02)$ were associated with decreased risk of mortality, hence increased survival compared to other primary tumors histology at 3-months of DS.

Conclusion: In our analysis, the presence of more than 1 extraspinal metastasis and poor preoperative neurology $(A-B-C)$ were poor prognostic factors of survival in patients with MSCC. 
7:38 - 7:40 am

\section{Surgery and Radiation Therapy versus High Dose Definitive Radiation Therapy for the Treatment of Mobile Spine Chordomas}

Nida Fatima; Elie Massaad; Ganesh Shankar; John H. Shin MD

Introduction: The treatment of mobile spine chordomas is typically en bloc resection when feasible as intralesional resections are associated with higher rates of recurrence and poor survival. The main consideration for surgery is operative morbidity and the potential complications associated with excisional resections with margins. Given the various regional anatomic nuances affecting the feasibility of en bloc resection, radiation therapy in the form of protons, photons, and carbon ion therapy have been utilized as alternatives to surgery. For this reason, there is currently variability in how newly diagnosed chordomas of the mobile spine are treated.

Methods: A literature search was performed in compliance with Preferred Reporting of Systematic Review and Meta-Analysis from 1990-2019. Outcome measures included progression free-survival (PFS) at the last followup and overall survival (OS). Pooled estimates of PFS and OS was computed, and reported using $95 \%$ Confidence Interval $(\mathrm{Cl})$ with $\mathrm{p}<0.05$ considered statistically significant.

Results: With a median follow-up of 6.3 years (range, 33 months-14.5 years), 1,680 patients were included from 28 articles. Majority of the patients (89.9\%; males, $62.3 \%)$ were treated primarily with surgery+/- radiation therapy while $10.1 \%$ (males, $59.5 \%$ ) had high-dose radiation therapy as a part of initial management of mobile spine chordomas. The PFS at 5 to 10 -years was $69.9 \%$ vs $72.2 \%, p=0.65$ among patients with surgery \pm radiation therapy and high dose definitive radiation therapy respectively. However, patients treated with highdose radiation therapy had significantly longer OS compared to patients who underwent surgery $+/$ - radiation (OS rate $=84.90 \%$ vs $58.89 \% ; \mathrm{Cl} 95 \%=11.75-40.31 ; \mathrm{p}=0.001)$.

Conclusion: Our results suggest that patients with primary radiation therapy have better survival than surgery \pm radiation therapy. However, there does not exist difference in terms of PFS between the two groups. Further prospective studies are needed to validate our results. 


\section{7:40- 7:42 am}

\section{Identification of Factors Associated with Outcomes in Surgical Decompression for Metastatic Spinal Cord Compression}

Asad M. Lak MD; Amina Rahimi; Abdullah M. Abunimer MD; Ian Tafel MD; Sharmila Devi; Akash Premkumar; Fidelia Ida; Hasan A. Zaidi BS, MD

Introduction: MSCC can result in significant impairment in a patients QoL and often needs surgical intervention.

Methods: Retrospective chart review

Results: A total of 151 patients were included in this study. The mean age was 60.4 years. The five most common tumor types were; lung, multiple myeloma, renal, breast and prostate cancer. The median time from tumor diagnosis to cord compression was 12.0 months. $67.5 \%$ patients had single level cord compression and $49.3 \%$ patients were operated within 24 hours. The median EBL was $500 \mathrm{ml}$ and average LOS was 10.3 days. $17.8 \%$ of patients had post-op complications and the mean follow-up was 7 months. Pre-op presenting symptoms included motor weakness $(70.8 \%)$, pain $(70.1 \%)$, sensory disturbances $(47.6 \%)$ and bowel or bladder disturbance (31.1\%). Post-operatively, 58.3\% reported improvement, 31.5\% had no improvement and $10.0 \%$ had worsening of pre-op status. The mean QALY gained in the entire cohort per year was 0.55. QALY gained in the first six months was 0.1 and in the first year was 0.4 . For patients who lived 1-2 years, 2-3 years, 3-4 years and 4-5 years, the mean QALY gained was 0.8, 1.5, 1.9 and 2.6 respectively. Multivariate logistic regression identified pre-op motor weakness, bowel or bladder dysfunction and ASA class as independent predictors of outcome.

Conclusion: The benefits of surgical decompression following MSCC are directly correlated with the amount of time the patient lives. 
7:42 - 7:44 am

\section{Predictors of Neurological Recovery after Decompressive Surgery in Metastatic Spinal Cord Compression}

Nida Fatima; John H. Shin MD

Introduction: Metastatic spinal cord compression (MSCC) is a debilitating oncological disorder, and often associated with permanent neurological deficits.

Methods: A retrospective chart review of a prospectively maintained database of the patients who underwent DS for MSCC, at an academic tertiary care hospital, was carried out (2011-2018). Multivariate Cox proportional hazards regression model was used to identify the predictors and create the scoring system of neurological recovery after DS in MSCC.

Results: The study cohort included 119 patients (63\%males, median age:67 years) with a median clinical follow-up of 345 days (range,90-678 days). From the multivariate analysis, involvement of (>4) vertebrae (HR:0.47,95\% Cl:0.22-1.01; $p=0.05$ ] independently decreased the likelihood of neurological recovery to modified Frankel grade D-E by 53\%. However, the posterior approach (HR:14.2,95\%Cl:1.76-118.1;p=0.01) independently increased the likelihood of improved neurological recovery at the last follow-up evaluation by 14folds. For patients with sacral metastasis (HR:0.06,95\% Cl:0.05-0.75; $\mathrm{p}=0.02)$, and those with primary tumor histology of melanoma (HR:0.10,95\% Cl:0.03-0.33; $\mathrm{p}=<0.0001)$ and gastrointestinal $(\mathrm{HR}: 0.31,95 \% \mathrm{Cl}: 0.11$ $0.82 ; p=0.02$ ) had less likelihood of neurological recovery at the last follow-up examination.

Conclusion: The posterior approach during the surgical decompression improved neurological status (modified Frankel grade D-E), however, the presence of 4 spinal level of metastasis, sacral location, and primary tumor histology of melanoma and gastrointestinal were associated with poor neurological recovery among patients who underwent DS for MSCC 
7:44 - 7:46 am

\section{Predictive Ability of Metabolic Syndrome-ATP III For Survival and Complications in Metastatic Spinal Cord Compression}

Nida Fatima; John H. Shin MD

Introduction: Metabolic Syndrome (MetS) is a global epidemic disorder, especially in the western capitalistic economic development model.

Methods: Patients with MSCC presented at an academic tertiary care hospital from 2011 to 2018 were identified through retrospective chart review. Patients were categorized into MetS-ATP III $(42.9 \%, n=51)$ if they meet 3 or more of the following criterion: waist circumference (men $>102 \mathrm{~cm}$ and women $>88$ $\mathrm{cm}$ ), triglycerides ( $\geq 150 \mathrm{mg} / \mathrm{dl}$ ), high density cholesterol (men $<40 \mathrm{mg} / \mathrm{dl}$ and women $<50 \mathrm{mg} / \mathrm{dl}$ ), blood pressure $(\geq 130 / \geq 85 \mathrm{mmHg})$ and fasting glucose $(\geq 110 \mathrm{mg} / \mathrm{dl})$. Cox-Multivariate proportional hazards regression model was computed to determine the association of MetS-ATP III with postoperative outcomes. Kaplan-Meier survival curve with the log-rank test was used to visually illustrate the differences in survival between MetS patients.

Results: Statistical analysis included 119 patients (median age: 67 years; $63 \%$ males), with a median survival of 14 months $(95 \% \mathrm{Cl}: 1-28.2$ months, $p=0.01)$. All-cause mortality and complications were $62.2 \%(n=74)$ and $22.7 \%(n=27)$ in our cohort respectively. Patients with MetS-ATP III had 1.74-folds higher risk of mortality at last follow up evaluation ( $\mathrm{HR}: 1.74,95 \% \mathrm{Cl}: 1.07-2.81, \mathrm{p}=0.02$ ), after adjusting for age, sex, tumor histology and spine instability neoplastic score (SINS). Overall complication rates were significantly higher among patients with MetS (HR: 4.06, 95\% $\mathrm{Cl}$ : 1.68-9.82, $\mathrm{p}=0.002$ ). Furthermore, the patients with MetS had a higher probability of impaired wound healing (HR: $3.85,95 \% \mathrm{Cl}: 1.57-9.40, \mathrm{p}=0.003)$ and thrombotic disorders (HR: 5.56, 95\% Cl: 1.63-19.0, $\mathrm{p}=0.006)$, adjusted for age, sex, tumor histology and SINS.

Conclusion: MetS-ATP III analysis in patients with MSCC who underwent DS was effective in identifying patients at higher risk for shorter survival and more complications. However, further prospective studies are needed to validate our results. 
Spine Summit Meeting Abstracts, May 2021

7:46 - 7:48 am

\section{The Effect of Red Blood Cell Transfusion on Perioperative Morbidity after Metastatic Spinal Tumor Surgery}

Joshua Benton; Rafael De la Garza Ramos MD; Yaroslav J. Gelfand MD; Phillip C. Cezayirli MD; Murray Echt MD; Vijay Yanamadala MD, MBA; Reza Yassari MD, MS

Introduction: Patients undergoing surgery for spinal metastases often experience significant morbidity. We performed a retrospective analysis at a single institution to identify the impact of red blood cell (RBC) transfusion on perioperative complications after metastatic spinal tumor surgery (MSTS).

Methods: Adult patients with cord compression or instability from a metastatic tumor who underwent surgery between 2013 and 2019 were identified from our institutional database. Patient demographics, comorbidities, oncologic history, pre-operative laboratory results, surgical data, transfusion events, and perioperative complications (within 30 days) were collected. Patients were considered to have received a RBC transfusion if it occurred intraoperatively or within 96 hours post-operatively. Multiple logistic regression was used to assess the independent effect of RBC transfusion and transfusion volume on our primary outcome.

Results: A total of 105 patients were identified from our database. The median age was 61.3 years, and $58.1 \%$ of patients were male. Thirty-four patients $(32.4 \%)$ experienced at least one perioperative complication. The most common complications patients experienced were infectious (14.2\%), thromboembolic events (13.2\%), and 30-day mortality (4.7\%). On multiple logistic regression analysis after controlling for age, sex, hypertension, radiographic evidence of spinal cord compression, Frankel grade, Eastern Cooperative Oncology Group status, and Tokuhashi scores, we found that RBC transfusion (OR 3.17, 95\% Cl 1.16 - 9.35, $p=0.029)$ and a preoperative Karnofsky score $<70$ (OR 4.62, 95\% Cl $1.26-18.45, p=0.023)$ were both independent predictors of perioperative complications. Secondary analysis also showed an independent and significant increase in complications with increasing RBC transfusion volume (OR 7.29, 95\% Cl 2.48 - 29.54, $\mathrm{p}=0.002$ ).

Conclusion: In this study, RBC transfusion and a preoperative Karnofsky score less than 70 were found to significantly increase the risk of perioperative complications after MSTS. 
Spine Summit Meeting Abstracts, May 2021

7:48 - 7:50 am

\section{Operative Predictors of Discharge Disposition, Hospital Stay Duration, and Expenditures After Intradural Tumor Removal}

Michael C, Jin; Allen L. Ho MD; Atman Desai MD, MA

Introduction: Intradural-extramedullary spinal tumors are rare and how operative variables affect short- and long-term outcomes are not well established.

Methods: IBM MarketScan Claims Database was queried for adult patients receiving surgery for intradural extramedullary tumors between 2007 and 2016. Multiple regression was used to evaluate factors associated with prolonged post-surgical inpatient stay, hospital costs, and 90-day post-discharge costs. Multivariable logistic regression was used to assess factors associated with discharge to continued care (defined as rehab, SNF, or another healthcare facility).

Results: A total of 4,573 adult patients underwent removal of intradural-extramedullary tumors. Most surgeries utilized a posterior approach $(n=4,467,97.7 \%)$ and tumors were most commonly found in the lumbar region $(n=1,851,40.5 \%)$, followed by the thoracic $(n=1,716,37.5 \%)$ and cervical $(n=911,19.1 \%)$ regions. In total, 970 $(21.2 \%)$ were discharged to continued care, frequently to either inpatient rehabilitation $(n=404,8.8 \%)$ or to a skilled nursing facility $(\mathrm{n}=177,3.9 \%)$.

Microscope use was independently associated with decreased need for continued care after discharge $(\mathrm{OR}=0.71, p<0.001)$ while older age $(\mathrm{OR}=1.03, p<0.001)$, female sex $(\mathrm{OR}=1.35, p<0.001)$, and malignant tumors (OR=1.61, $p=0.001$ ) were associated with increased likelihood for needing continued care. While anterior approach was independently associated with increased duration of post-surgical hospitalization $(b=2.32, p<0.001)$ and increased index admission cost $(b=42213.42, p<0.001)$, it was not associated with differences in discharge disposition $(\mathrm{OR}=1.22, p=0.399)$ or 90 -day healthcare costs $(\mathrm{b}=-397.78, p=0.898)$. Microscope use was also associated with decreased post-surgical hospital stay duration $(b=-0.77, p<0.001)$, index admission costs $(b=-6465.02, p<0.001)$, and 90-day healthcare costs $(b=-5340.70, p<0.001)$. Tumor histology was not associated with discharge disposition, short-, or long-term costs.

Conclusion: Anterior surgical approach was associated with longer hospital stays and hospital costs but did not affect discharge disposition or long-term healthcare costs. Microscope use was associated with improved discharge disposition, shorter hospital stays, and lower short- and long-term costs. 
7:50 - 7:52 am

\section{Variation in Opioid Prescribing Patterns and Impact on Refill Prescriptions Following Elective Lumbar Spine Surgery}

Anshit Goyal MBBS; Stephanie Payne; Lindsey Sangaralingham; Mohammed A. Alvi MD; Molly Jeffery; James Naessens; Halena Gazelka ; Elizabeth B. Habermann PhD; Benjamin D. Elder MD, PhD; Robert J. Spinner MD; Mohamad Bydon MD

Introduction: With a dramatic rise in prescription opioid use over the last decade, it is imperative to understand post-surgical prescribing patterns and their impact on persistent opioid use.

Methods: The OptumLabs Data Warehouse(OLDW) was queried for postdischarge opioid prescriptions for patients undergoing the following elective lumbar spine procedures for degenerative spine disease-, posterior lumbar fusion(PLF), anterior lumbar fusion(ALF); circumferential lumbar fusion(CLF) and lumbar decompression(LD);grouped by US Census Division. Mean total morphine milliequivalents(MMEs) adjusted for age and sex were calculated for each procedure type by census division. Frequency of overprescribing(defined either as total MMEs>200 or length of supply>7 days, per CDC guidelines) was also calculated.

Results: A total of 43,438 patients with 39,760 postdischarge opioid prescriptions were included, with the majority following PLF $(43 \%, n=17,355)$ or $\operatorname{LD}(39 \%, n=15,471)$. There was wide variation in adjusted total prescribed mean MMEs across all census divisions for each procedure (ALF: 767-1156, CLF: 910-1445, PLF: 713-1260 and LD: 503-745). Across all census divisions, a significant proportion of cases were found to be overprescribed in terms of total MMEs (ALF: $87 \%$ to $95.6 \%$; CLF: $59 \%-94.2 \%$; PLF: $81.4 \%$ to $96.5 \%$; LD: $80.5 \%$ to $90.9 \%)$. Multivariable analysis showed that following an index fill, opioid-naïve(OR: $0.37(0.34-0.40)$, $p<0.001)$, Hispanic(OR: 0.86 (0.76-0.98), $p<0.001)$, elderly patients $>65$ years (OR: $0.54(0.44-0.67), p<0.001)$ and those prescribed short acting opioids $(0.67(0.59-0.76), p<0.001)$ were less likely to seek an opioid refill while females (OR: 1.14(1.07-1.22), $p<0.001)$ and patients undergoing inpatient surgery(OR: 1.25(1.11-1.40), $\mathrm{p}<0.001$ ) were more likely to seek a refill prescription within 6 weeks of a PLF.

Conclusion: There is wide variation in postdischarge opioid prescribing patterns following elective lumbar spine surgery, with a significant proportion of patients being overprescribed. Females and patients undergoing inpatient surgery are more likely to seek opioid refills within 6 weeks of surgery. 
7:52 - 7:54 am

\section{Risk Factors for Dysphagia following Posterior Cervical Fusion: A 10-year Single Institutional Experience}

Nida Fatima; John H. Shin MD

Introduction: Severe and disabling dysphagia has commonly been attributed as a complication of anterior cervical discectomy and fusion, however, the current literature is still scarce regarding dysphagia following posterior cervical fusion (PCF).

Methods: Patients who underwent PCF were retrieved from a prospectively maintained database over the period of 10-years at an academic tertiary care hospital. Dysphagia numeric rating scale was used for pre and postoperative assessment of dysphagia. Cox-Proportional Hazards Regression model was computed to determine the risk factors associated with dysphagia following PCF.

Results: Our cohort included 199 patients (50.8\% females, mean age: $65.5 \pm 12.5$ years), with a median clinical follow-up of 361 days. Postoperatively, 11 patients (5.5\%) developed dysphagia. Patients with postoperative dysphagia had significantly greater history of preoperative swallowing difficulty $(p<0.001),{ }^{35}$ fixation range $(p=0.01)$, and revision surgery $(p=0.01)$. The postoperative radiographic alignment parameters and correction in these angles were not significantly associated with the occurrence of dysphagia. Multivariable regression analysis revealed that preoperative dysphagia had 20.6-folds higher risk of developing postoperative dysphagia in patients with the PCF (HR: $20.6,95 \% \mathrm{Cl}: 2.46-171.5, \mathrm{p}=0.005)$. Furthermore, among patients with ${ }^{3} 5$ levels of PCF and instrumentation had 4.8-folds higher risks of postoperative dysphagia (HR: $4.87,95 \% \mathrm{Cl}$ : 1.15-20.6, $p=0.03)$.

Conclusion: Regardless of the surgical corrective angles, the preoperative history of dysphagia and increased number of posterior levels fused were independent risk factors for postoperative dysphagia. 


\title{
7:54 - 7:56 am
}

\section{Long-Term Assessment of Post-Surgical Opioid Use in Patients after Resection of Intradural- Extramedullary Tumors}

\author{
Allen L. Ho MD; Michael C. Jin; Atman Desai MD, MA
}

Introduction: Despite efforts to better control pharmacological dependency among spine surgery patients, the risk factors predisposing patients receiving intradural tumor resection to long-term use remain unclear.

Methods: Adult patients receiving spine surgery for resection of intradural-extramedullary tumors between 2007 and 2015 were identified in the IBM MarketScan Claims Database. Only patients with at least 365 days of continuous post-discharge follow-up were included. Multiple linear regression and multivariable logistic regression were used to assess pharmaceutical prescribing practices of opioids, benzodiazepines, and skeletal muscle relaxants (SMR) following discharge. Long-term use was defined by the presence of at least 6 prescriptions during the 12 months following surgery.

Results: A total of 2,749 patients receiving resection of intradural extramedullary tumors were eligible. The majority of surgeries were anterior approach $(n=2,694,98.0 \%)$ and most tumors were benign $(n=1,736$, $90.3 \%)$. A minority of patients received arthrodesis after tumor removal $(n=295,10.7 \%)$.

On multivariable analysis, arthrodesis was associated with increased post-discharge opioid $(b=1.14, p=0.002)$ and benzodiazepine $(b=0.37, p=0.025)$ prescriptions. Anterior approach was associated with decreased benzodiazepine $(b=-0.75, p=0.37)$, but not opioid $(b=-0.18, p=0.819)$, prescribing. Neither arthrodesis nor anterior approach were associated with SMR prescribing after surgery. After adjusting for available covariates, a decrease in long-term opioid and benzodiazepine use between 2007 and 2015 was observed, likely due to specialty-wide changes in opioid prescribing practices (continuous; opioid $\mathrm{OR}=0.95, p=0.019$ ); benzo $\mathrm{OR}=0.91, p=0.032)$. However, patients receiving post-resection fusion remain at increased risk for long-term opioid $(\mathrm{OR}=1.41, p=0.045)$ and benzodiazepine use $(\mathrm{OR}=1.78, p=0.041)$. Notably, neither tumor malignancy nor tumor location were associated with differences in long-term opioid or benzodiazepine use.

Conclusion: While long-term opioid and benzodiazepine usage after spinal tumor resection appears to be decreasing, arthrodesis following intradural tumor removal remains an important risk factor for long-term use of both restricted drugs, regardless of tumor location or malignancy. 
7:56 - 7:58 am

\section{Bauer and Spinal Instability Neoplastic Scores as Predictors of Blood Transfusion After Metastatic Spinal Tumor Surgery}

Rafael De la Garza Ramos MD; Joshua Benton; Yaroslav J. Gelfand MD; Phillip C. Cezayirli MD; Murray Echt MD; Vijay Yanamadala MD, MBA; Reza Yassari MD, MS

Introduction: Patients undergoing surgery for spinal metastases often require red blood cell (RBC) transfusion intraoperatively or in the immediate post-operative period. Allogenic blood transfusions are associated with an increased risk of post-operative complications, making tools to predict and avoid the need for RBC transfusions in surgical patients of great importance.

Methods: Adult patients with cord compression or instability from a metastatic tumor who underwent surgery between 2013 and 2019 were identified from our institutional database. Patient demographic data, comorbidities, oncologic history, pre-operative labs, surgical data, and transfusion events were collected. Patients were considered to have received a RBC transfusion if it occurred intraoperatively or within 96 hours post-operatively. Univariate analysis and multiple logistic regression were used to compare data between patients who received RBC transfusions versus those who did not receive one. Multiple logistic regression was used to assess the effect of independent predictors on our primary outcome.

Results: A total of 105 patients were identified from our database. The median age was 61.3 , and $58.1 \%$ were male. Fifty patients (47.6\%) received at least one RBC transfusion. On multiple logistic regression controlling for age, gender, and all statistically significant variables on univariate analysis - Spinal Instability Neoplastic score (SINS) and Bauer scores - the preoperative SINS score (OR 1.18, 95\% Cl 1.01 - 1.40, $p=0.048$ ) and Bauer score (OR 0.60, 95\% Cl $0.39-0.91, p=0.019)$ were identified as independent predictors of patients requiring $\mathrm{RBC}$ transfusions.

Conclusion: Preoperative SINS and Bauer scores may serve as useful predictors of which patients undergoing surgery for spinal metastases will require RBC transfusions. 
Spine Summit Meeting Abstracts, May 2021

7:58 - 8:00 am

\section{Variation in Opioid Prescribing Patterns and Impact on Refill Prescriptions Following Elective Cervical Spine Surgery}

Anshit Goyal MBBS; Mohamad Bydon MD; Stephanie Payne; Lindsey Sangaralingham; Mohammed A. Alvi MD; Molly Jeffery; James Naessens; Halena Gazelka ; Elizabeth B. Habermann PhD; Benjamin D. Elder MD, PhD; Robert J. Spinner MD

Introduction: Understanding post-surgical prescribing patterns and their impact on persistent opioid use is important to establish effective and reasonable opioid prescribing protocols.

Methods: The OptumLabs Data Warehouse(OLDW) was queried for postdischarge opioid prescriptions for patients undergoing the following elective cervical spine procedures for degenerative spine disease-anterior cervical discectomy and fusion(ACDF), posterior cervical fusion(PCF), circumferential cervical fusion (CCF) and cervical decompression(CD); grouped by US Census Division. Mean total morphine milliequivalents(MMEs) adjusted for age and sex were calculated for each procedure type by census division. Frequency of overprescribing(defined either as total MMEs $>200$ or length of supply $>7$ days, per CDC guidelines) was also calculated.

Results: A total of 21,947 patients were included with the majority undergoing ACDF $(78 \%, n=17,298)$. There was wide variation in adjusted total prescribed mean MMEs across all census divisions for each procedure(ACDF: 518-863, CCF: 696-2138, PCF: 724-1449;CD: 551-961). Across all census divisions, a significant proportion of cases were found to be overprescribed in terms of total MMEs(ACDF: $84 \%$ to 93.2\%;CCF: $63 \%-94.2 \%$;PCF: $78.6 \%$ to $95 \%$;CD: $81.2 \%$ to $94.2 \%$ ). Multivariable analysis showed that after an index fill, opioid-naïve(OR: 0.30 (0.28-0.32),p<0.001), Hispanic (OR: 0.84 (0.73-0.95), $p<0.001)$, elderly patients $>65$ years(OR:0.52(0.42-0.65), $p<0.001)$ and those prescribed short acting opioids $(0.50(0.42-0.60), p<0.001)$ were less likely to seek an opioid refill while females(OR: 1.17(1.10-1.25),p<0.001) and patients undergoing inpatient surgery(OR: $1.16(1.09-1.24), p<0.001)$ were more likely to seek a refill prescription within 6 weeks of an ACDF.

Conclusion: In analysis of fill data, we observed that there was significant overprescribing and wide variation postdischarge opioid prescribing patterns following elective cervical spine surgery. Females and patients undergoing inpatient surgery were more likely to require refill prescriptions. 
8:00 - 8:02 am

Outcomes after Decompressive Surgery in patients with Metastatic Spinal Cord Compression: A 70,734 Person-year Study

Nida Fatima; John H. Shin MD

Introduction: Surgical decompression is considered as a palliative care from oncological perspective for patients with metastatic spinal cord compression (MSCC).

Methods: A retrospective cohort study was performed using the electronic database at an academic tertiary care hospital for the patients who underwent DS for MSCC from January 2011-December 2018. Fischer exact test was used to examine difference between the groups, and Kaplan-Meier Estimate was plotted for survival analysis.

Results: Our cohort included 119 patients with $75 \%$ males $(n=63)$, with a median age of 67 years (range, 3384 years). The median time to intervention (DS) from primary tumor diagnosis was 2.4 years (range, 1 day-32 years), with the lung adenocarcinoma being the most common primary tumor histology (19.3\%) metastasizing to thoracic vertebrae in $70.6 \%$ of the patients. Majority of the patients $(93.3 \%)$ underwent posterior surgical approach in the form of laminectomy $(46.2 \%, n=55)$ and vertebrectomy $(53.8 \%, n=64)$, either within 48 hours $(57.1 \%, n=68)$ or $\geq 48$ hours $(42.8 \%, n=51)$. Overall, thirty-five patients $(29.4 \%)$ improved to modified Frankel Grade E postoperatively, whereas 2 patients (1.7\%) deteriorated to modified Frankel Grade A. Thirty-two patients $(26.8 \%)$ developed complications, which included wound complications and dehiscence $(10.1 \%$, $n=12)$, deep venous thrombosis and pulmonary embolism $(12.6 \%, n=15)$, and instrumentation failure $(4.2 \%$, $\mathrm{n}=5)$. The median overall survival in our cohort was 345 days $(95 \% \mathrm{Cl}$ : 91.77-652.8 days).

Conclusion: Our analysis indicate that functional status and survival improved in patients after DS in MSCC. 
Spine Summit Meeting Abstracts, May 2021

8:02 - 8:04 am

\section{Thirty- and 90- Day Readmissions after Spinal Surgery for Spine Metastases: A National Trend Analysis}

Aladine A. Elsamadicy BE; Andrew B. Koo ; Wyatt David; Cheryl Zogg; Adam Kundishora MD; Christopher S. Hong MD; Gregory A. Kuzmik MD; Ramana Gorrepati MD; Pedro O. Coutinho MD; Luis Kolb MD; Maxwell Laurans; Khalid M. Abbed MD

Introduction: Unplanned 30- and 90-day readmissions after major surgery are increasingly being valued as important markers of quality of care. The 30- and 90-day rates for readmission following surgery for spine metastases have not been well described.

Methods: The Nationwide Readmission Database years 20132015 was queried.Unique patient linkage numbers were used to follow patients and identify 30- and 31 to 90-day readmission rates. Patients were grouped by no readmission (Non-R), readmission within 30 days (30-R), and readmission within 31 to 90 days (90-R). Weighted multivariate analysis assessed impact of treatment approach and clinical factors associated with 30- and 90-day readmissions.

Results: There were a total of 4,423 patients with a diagnosis of spine metastases identified who underwent spine surgery, of which $1,657(37.5 \%)$ encountered either a $30-(24.1 \%)$ or 90 -day $(13.3 \%)$ unplanned readmission. The most prevalent inpatient complications observed were postoperative infection (30-R: $16.3 \%$, 90-R: $14.3 \%$, Non-R: $11.5 \%$ ), acute post-hemorrhagic anemia (30-R: 13.4\%, 90-R: 14.2\%, Non-R: 14.5\%), and genitourinary complication (30-R: 5.7\%, 90-R: 2.9\%, Non-R: 6.2\%). The most prevalent 30-day and 90-day reasons for admission were sepsis (30-R: 10.2\%, 90-R: 10.8\%), postoperative infection (30-R: 13.7\%, 90-R: $6.5 \%)$ and genitourinary complication (30-R: $3.9 \%, 90-R: 4.1 \%)$. On multivariate regression analysis, surgery type, age, hypertension, and renal failure were independently associated with 30-day readmission; rheumatoid arthritis/collagen vascular diseases and coagulopathy were independently associated with 90-day readmission.

Conclusion: In this study, we demonstrate that readmissions after surgical treatment intervention for spine metastases are common. Furthermore, we find that the most common reasons for readmission are sepsis, postoperative infection and genitourinary complications. We found few patient-level factors were independently associated with hospital readmission. 
8:04 - 8:06 am

\section{Differential Bone Marrow Immune Landscape May Foster Permissive Tumor Growth in the Spine}

Michael J. Strong MD, PhD; Aqila Ahmed; Sabrina Rocco; Anda-Alexandra Calinescu; Nicholas J. Szerlip MD, FACS

Introduction: Roughly 400,000 people have bone metastases in the U.S. and the vast majority of these occur in the spine. Metastases to the spine, result in fractures, pain, paralysis, and enormous health care costs. This predilection is not fully understood. The seed and soil theory posits that differences in local bone microenvironment may account for this differential growth pattern. Further, the immune microenvironment has been shown to have a large impact on tumor growth and progression.

Methods: A total of 10 spines and femurs from male C57BL6/J mice were harvested and processed for immunophenotyping using Mass Cytometry by Time-Of-Flight (CyTOF) and stained for 33 surface protein markers using a mouse antibody panel. We also collected and processed 3 spine and 2 femurs from human and analyzed them with CyTOF using the Maxpar Complete Human T cell Immuno-Oncology Panel Set (Fluidigm).

Results: There are global shifts in immune cell composition between the long bone and spine microenvironment. In the long bone, there was an increase in neutrophils and monocytes; however, in the spine, there was an elevation of myeloid-derived suppressor cells (MDSCs). Although there are general differences between the mouse and human immune systems, we were able to observe similar trends in our human data with a predominance of MDSCs in the spine compared to long bone marrow.

Conclusion: There appears to be an immunosuppressive signature that is specific to vertebral bodies compared to the long bone that may partial contribute to the metastatic permissive growth seen in spine. 
8:06 - 8:08 am

\section{Use of Preoperative Nutrition Consults Predicts Fewer Complications and Faster Discharge after Spine Metastasis Surgery}

Jeffrey S. Ehresman BS; Andrew Schilling; A. Karim Ahmed; Zach Pennington BS; Daniel Lubelski MD; Ethan Cottrill; Matthew Goodwin; Alexandra Liddy; Nancy A. Abu-Bonsrah MD; C. Rory Goodwin MD, PhD; Daniel M. Sciubba MD

Introduction: Preoperative malnutrition is associated with increased postoperative complications rates and hospital length of stay. However, the degree to which these risks can be mitigated by nutritional consultation has not been well-described.

Methods: Adult patients who underwent neurosurgical intervention for spinal metastases at a tertiary care institution between 2005 and 2016 were retrospectively reviewed. Stepwise multivariable linear regression analyses were used to identify associations with 30-day complication rates and hospital length of stay.

Results: Among 95 patients who met inclusion criteria, average length of stay was 8.9 days and $39(41.1 \%)$ experienced one or more postoperative complications. On multivariable analysis, thirty-day complication rates were higher in the absence of a preoperative nutrition consult, a Modified Charlson Comorbidity Index (MCCl) of greater than 2 points, greater operative blood loss, and malnutrition (Nutritional Risk Index below 97.5). Further, hospitalization duration was increased with the absence of a nutrition consult, malnutrition, congestive heart failure, and prior systemic therapy in the multivariable analysis.

Conclusion: On multivariable analysis, receipt of a preoperative nutrition consult was the only factor associated with both decreased 30-day complication rate and shorter hospitalization. We therefore posit that greater implementation of nutritional counseling may help to decrease complication rates and expedite discharge in patients undergoing surgical intervention for spinal metastases. 
8:08 - 8:10 am

\section{Application of Cooperative Game Theory Principles to Interpret Machine Learning Models of Readmission in Spine Surgery}

Michael L. Martini BA; Eric K. Oermann MD; Sean N. Neifert BS; Jonathan Gal; Dominic A. Nistal BA; John M. Caridi MD

Introduction: Unplanned hospital readmissions present a substantial cost burden on the healthcare system. Previous studies identified factors implicated in readmission risk, however, it is unclear how interaction effects between clinical factors may modulate overall readmission risk.

Methods: A total of 396 patients (3.6\%) experienced a 30-day unplanned readmission of the 11,150 reviewed cases involving procedures for degenerative spine conditions. Gradient boostingclassifiers were used to construct predictive patient-level readmission models. Shapley values, which apply an optimal cost-sharing rule to assign a unique distribution of the total readmission risk to each factor in the model, were used to quantify feature importance and examine interaction effects between variables across the study population.

Results: Gradient boosting models constructed from features identified by optimized Shapely values performed well in predicting patient-level readmission risk (mean C-statistic=0.72). While length of stay and age were the most important factors across all models, Shapley interaction analysis revealed that transfer admission status significantly modifies the importance of several variables in readmission prediction. These include positive interactions with sex, comorbidity burden, and surgical duration, and negative interaction effects with age.

Conclusion: This strategy provides novel insights into global patterns of feature interactions that contribute to readmission risk in spine surgery. Certain patient subgroups with multiple positively interacting risk factors, such as transfer admissions, may require special attention during their stay to control readmission risk. 
8:10 - 8:12 am

\section{Machine Learning-Based Feature Importance Analysis Enhances Prediction of Nonroutine Discharge Following Spine Surgery}

Michael L. Martini BA; Eric K. Oermann MD; Sean N. Neifert BS; Jonathan Gal; Dominic A. Nistal BA; John M. Caridi MD

Introduction: Nonroutine discharge (NRD) is an important source of rising healthcare costs. Identifying factors contributing to NRD risk presents opportunities to reduce costs and improve quality of care.

Methods: From 11,150 reviewed cases involving surgery for degenerative spine conditions, 1,764 (15.8\%) involved NRD. Over 75 pre-discharge variables were collected and categorized into feature domains pertaining to demographic, perioperative, and postoperative factors. Multilayer perceptron neural networks were used to construct predictive models for NRD from feature domains. An ensemble tree-specific method quantified and ranked features by relative importance.

Results: In the demographics domain, age and comorbidity burden were the most important features for NRD prediction. Surgical duration, urine output, intraoperative oral morphine equivalents, and estimated blood loss were the most important perioperative features. Hospitalization cost was the most important postoperative factor for predicting NHD. Supervised learning experiments showed that the postoperative events model performed the best alone $(\mathrm{C}$-statistic $=0.85)$ while the perioperative model performed the worst $(\mathrm{C}$ statistic=0.79), suggesting that postoperative events may contribute more to NRD risk than perioperative or intraoperative variables. An enriched model using only the most important features significantly improved predictive capacity $(\mathrm{C}$-statistic $=0.91)$. A threshold of 0.27 optimized the models precision-recall tradeoff $(\mathrm{F} 1$ score $=0.65$ ).

Conclusion: This study provides insight into potential drivers of spine surgery NRD and suggests that importance analysis with feature domains is a valuable method for institutions to construct high-performing machine learning models for predicting clinical outcomes. 
8:12 - 8:14 am

\section{Spine Surgery is Safe and Effective in the Extremely Elderly; An Ambispective Study of 722 Patients Over 75 Years Old}

Jamie RF Wilson BA.BM.BCh(OXON).FRCS(Neurosurgery); Jetan H. Badhiwala; Robert Ravinsky; Hetshree Joshi; Omar Khan; Fan Jiang MD; Raja Y. Rampersaud MD, FRCS(C); Stephen Lewis MSc, MD, FRCS(C); Eric M. Massicotte MD; Michael G. Fehlings MD, PhD, FRCS(C), FACS

Introduction: Degenerative spine disease is an emerging epidemic in the elderly age group, however a number of important questions remain regarding the effectiveness and safety of complex spine procedures in those over 75 years old.

Methods: Prospectively-collected database records from a single tertiary spine care center were retrospectively interrogated from January 1st 2005 until July 31st 2018 to identify all patients over the age of 75 years. Descriptive demographics were collected, including type of surgery, region of surgery, operated levels, perioperative complications and patient reported outcome measures, amongst others. Multivariate regression analysis was performed to compare increasing age with the change of complications by region, number of levels, rate of revision surgery, and outcomes at 1 month and 1 year.

Results: 722 patients were included, with an age range of 75 - 92 years old (mean 78.36 ). 57\% underwent lumbar region surgery, $27 \%$ cervical and $16 \%$ thoracic/occipital. In total, 412 cases (57\%) underwent instrumented fusion surgery, with the most common indication being degenerative stenosis. $43 \%$ of all cases were 1 or 2 level surgery, with $57 \%$ including 3 or more levels. Perioperative complications (total) occurred in $16 \%$ of cases, with $93(13 \%)$ revision cases performed in the follow up period. Multivariate analysis demonstrated an increasing likelihood of fewer levels of surgery as age increased $(p=0.009)$. The rate of complications at 1 year did not vary with region of surgery $(p=0.773)$ and did not increase with increased number of levels $(p=0.265)$, however, increasing age was associated with increased risk of revision surgery (adjusted for region and number of levels; $p=0.009$ ).

Conclusion: Complex spine surgery at any anatomical region, including instrumented fusion surgery, can be effectively administered in the extremely elderly patient population. However, increasing age is associated with an increased risk of revision surgery and this should be considered during pre-operative decision making. 
8:14 - 8:16 am

\section{Preoperative Factor Evaluation in Patients Undergoing Elective Spine Surgery: Results from a Single- Center Online Survey}

Kyle Mueller MD; Matthew R. D'Antuono BS; Nathan Nair MD

Introduction: Preoperative factor consideration to minimize complication avoidance following elective spine surgery is a part of clinical practice and resident education.

Methods: A 10-question online survey was sent to faculty and residents of a single center institution. Results were blinded. Respondents were asked whether they would delay or cancel an elective spine surgical case if a certain preoperative factor or radiographic image was abnormal or not obtained, respectively, at the time of the clinic visit, absent myelopathy or weakness. Preoperative factors considered were related to behavioral health (PHQ-9), nutritional status (Albumin/Pre-albumin), infection risk (hemoglobin A1C), bone quality (Vitamin D, DEXA), radiographic planning $(C T, 36$ " standing films, XR with plain and dynamic views) and baseline standard controls (MRI, CBC/BMP).

Results: $67 \%$ (18/27) of respondents completed the survey. Residents were statistically more likely to evaluate PHQ-9, albumin/pre-albumin, DEXA, CT and XR's in patients undergoing posterior cervical fusions and multilevel lumbar fusions $(p<0.05)$. No statistical difference was seen regarding the evaluation of MRI, hemoglobin A1C, vitamin D or 36" standing films.

Conclusion: A difference exists between clinical practice and trainee education regarding necessary preoperative data that should be acquired to minimize complications in elective spine surgery, specifically regarding multi-level spinal fusions. Standardized preoperative planning data collection processes may facilitate increased use of data in surgical planning and complication reduction. Further study on into the difference between practice standard and resident education is warranted. 
8:16 - 8:18 am

\section{The Impact of Preoperative Opioid Use on Patient Satisfaction in the Outpatient Spine Surgery Setting}

Nicholas M. Rabah BS; Hammad A. Khan BS; Jay M. Levin BA; Robert Winkelman BS; Thomas E. Mroz MD; Michael P. Steinmetz MD

Introduction: The Clinician and Group Consumer Assessment of Healthcare Providers and Systems (CGCAHPS) survey was developed as a result of the value based purchasing initiative by the Center for Medicare and Medicaid Services. It allows patients to rate their experience with their provider in the outpatient setting. With the prevalence of opioid abuse continuing to increase, many laws are being established to limit the number of opioids that can be prescribed for acute pain. For spine surgeons, this may mandate a change in practice, leaving many providers wondering how these new limitations might affect the patient experience after surgery.

Methods: All patients who had underwent lumbar spine surgery between 2009 and 2017 who completed a CGCAHPS survey after their procedure were studied. Patients who were prescribed an opioid at their postoperative visit created one cohort, while those who did not comprised the other. The two cohorts were then compared using a chi-square analysis based on the percentage of patients who selected a 9 or 10 on the overall provider rating (OPR) question, which is considered a top-box response.

Results: Our study population included 456 patients who had underwent lumbar spine surgery and completed a postoperative CGCAHPS survey. Of those patients, $80(17.5 \%)$ received a prescription for opioids at their postoperative visit and $376(83.5 \%)$ did not. Of those patients receiving opioids at their visit, $63(78.7 \%)$ indicated a top-box OPR. This is in comparison to the $334(89 \%, p<0.001)$ who did not receive opioids but selected a top-box OPR.

Conclusion: In the context of new opioid prescribing limitations, the results of the present study are encouraging that spine surgeons can decrease their opioid prescribing habits, while possibly improving satisfaction rates. 
8:18 - 8:20 am

\section{Value of the Hospital Frailty Risk Score for Predicting Adverse Postoperative Outcomes in Spinal Surgery}

Theodore Hannah BA; Sean N. Neifert BS; Jonathan Gal; Michael Martini ; Alexander J. Schupper; Trevor Hardigan MD; John Caridi

Introduction: As spine surgery becomes increasingly common in the elderly, frailty indices are being used to risk stratify patients. The recently published Hospital Frailty Risk Score (HFRS) is a novel method of assessing frailty using International Classification of Diseases, Tenth Revision (ICD-10) codes. However, HFRS utility has yet to be evaluated in spinal surgery.

Methods: Patients undergoing elective spine surgery at a single institution from 2008 to 2016 were reviewed. Those undergoing surgery for tumors, traumas, and infections were excluded. The HFRS was calculated for each patient, and rates of adverse events were calculated for low, medium, and high frailty cohorts. Predictive ability of the HFRS for various outcomes was also assessed using a model containing other relevant variables.

Results: ICU stays were more prevalent in high HFRS patients $(66 \%)$ than medium (31\%) or low $(7 \%)$ HFRS patients. Similar results were found for non-home discharges and 30-day readmission rates. Logistic regressions showed HFRS significantly improved the accuracy of predicting ICU stays (AUC=0.80), non-home discharges $(A \cup C=0.81)$, and total complications $(A \cup C=0.81)$. HFRS was less effective at improving predictions of 30-day readmission rates $(A \cup C=0.64)$ and $E D$ visits $(A \cup C=0.57)$.

Conclusion: HFRS is a better predictor of LOS than readmission and may improve on mFI in predicting LOS. Since ICU stays and non-home discharges are known to be the main drivers of cost variability in spine surgery, HFRS may be a valuable tool for cost prediction in this specialty. 
8:20 - 8:22 am

\section{A Multidisciplinary Spine Surgical Indications Conference Leads to Alterations in Surgical Plans in a Significant Number of Cases}

Joshua Benton; Rafael De la Garza Ramos MD; Yaroslav J. Gelfand MD; Phillip C. Cezayirli MD; Murray Echt MD; Reza Yassari MD, MS; Vijay Yanamadala MD, MBA

Introduction: Determining the optimal spine procedure for patients can often be difficult. Multidisciplinary decision making has previously demonstrated optimization of surgical planning. The input of multiple spine specialists may significantly change and improve this decision making process.

Methods: We reviewed consecutive cases at our institutions weekly spine surgery indications conference from September - October 2019 where three neurosurgical and three orthopedic spine surgeons discuss each upcoming surgery. The initial surgical decisions were documented prospectively. Every elective surgery at the institution was presented and each surgeon voiced their opinion before a group consensus decision was made on how to proceed. Patient demographics, comorbidities, surgeon specific information, the proposed surgery, invasiveness rating, and group consensus for each surgery were collected. Descriptive statistics were performed for the groups decision to accept or alter a proposed surgery. Univariate analysis was performed using chi-squared tests and Fishers exact test for categorical variables and t-tests and Wilcoxon rank-sum test for continuous variables to compare differences between surgical cases approved without changes and those that the group altered

Results: A total of fifty-five consecutive cases were reviewed. The median age was 60 and $30.9 \%$ of patients were male. Eleven cases (20\%) were altered based upon group consensus. Analysis with a chi-squared test indicated that the multidisciplinary group altered a statistically significant number of surgeries $\left(X^{2}=12.22\right.$, $p<0.001)$. The group suggested alterations that increased the surgery's invasiveness in five cases $(45.5 \%)$ and decreased the invasiveness or canceled the other six cases $(54.5 \%)$. No other variables were associated with an increased incidence of procedure alteration.

Conclusion: Multispecialty indication conferences may improve the spine surgery planning process by offering surgeons feedback on their surgical decision and allowing them to optimize their surgical plan. 
8:22 - 8:24 am

\section{Perioperative and Long-Term Opioid Use in Spine Surgery: An Analysis of the Medical Expenditure Panel Survey Data}

Luke Wooster; Robert Winkelman BS; Dominic Pelle MD; Thomas Mroz; Michael P. Steinmetz MD

Introduction: While prior studies have characterized the risk of long-term opioid use in the general population, the risk of long-term opioid use after spine surgery is not as clearly understood. Given that the field of spine surgery has been shown to have some of the largest postoperative opioid doses of all surgeries, an accurate assessment of the risks of long-term opioid use in this population may improve the development and implementation of opioid-related policy.

Methods: Information related to inpatient surgery admissions and opioid prescriptions were gathered from the 2009-2016 annual medical expenditure panel survey (MEPS) database. Spine surgery admissions (defined by IPC codes IPCCC1X-4X) who underwent an operation $(n=198)$ were compared against all other hospitalized patients who underwent an operation $(n=6031)$. Opioid use was assessed at each round of the survey (every 6 months) and chronic opioid use was defined as a filled opioid medication at least six months after index surgery.

Results: Overall prevalence of chronic opioid use in patients following inpatient spine surgery was higher than that in patients undergoing other inpatient surgeries ( $26 \%$ vs. $9.1 \%)$. The higher prevalence was observed across all time points between 2009 and 2016. Additionally, opioid use in the six months prior to surgery was observed in $34 \%$ of spine surgery patients and was associated with significantly greater odds of developing chronic use (OR = $26.7(8.0-89.5))$.

Conclusion: The prevalence of chronic opioid use following inpatient spine surgery is considerably higher than the prevalence of chronic opioid use in patients undergoing other inpatient operations. Additionally, opioid use prior to surgery was found to increase the risk of chronic opioid use following spine surgery. 
8:24 - 8:26 am

\section{Reducing Wrong Level Spinal Surgeries Through Root Cause Analyses: Analysis of an Institution's Iterative Policy Changes}

Shaarada R. Srivatsa BA; Shaleen Vira; Ajit A. Krishnaney MD, FAANS

Introduction: There is currently no protocol in the literature that guarantees elimination of wrong level spinal surgery (WLSS). Prior studies are limited to multi-institution surgeon surveys and opinion works, thus completely eliminating WLSS remains a challenge.

Methods: We retrospectively reviewed all wrong site spine surgeries and subsequent iterative prevention strategies employed at our institution between 2008 and 2019. The institutional database of wrong site surgeries was queried, and all corresponding WLSS-related RCA's were collected from institutional records. We conducted a longitudinal analysis of these reports and tracked policy implementations that resulted along with the incidence of WLSS resulting from each policy change.

Results: 15 WLSS were identified with 13 corresponding RCAs out of estimated21,179 spine surgeries between 2008-2019. Three broad policy categories emerged: imaging, operating room culture, and vertebral body marking. The longest-lasting changes from each category were: requiring two unmistakable, immovable vertebral markers (2013); requiring intraoperative radiographs with two markers and retractors in place (2014); open-ended questioning during spinal level verification by residents and fellows (2015); and requiring an impartial out-of-room radiologist to have verbal contact with the operating surgeon intraoperatively to collaboratively discuss localization for each case (2018). Each change was met with a decline in WLSS (5 in 2014, 3 in 2015, and 0 in 2019).

Conclusion: As evidenced by the slow progression of these stepwise guidelines, no one policy is effective in eliminating WLSS. Iterative policy changes in verbal communication between operating room staff and surgeon and radiologist, as well as improvements in intraoperative imaging and marking contributed to improved WLSS rates. By focusing on lessons learned from RCA's using this methodology, institutions can iteratively improve their rate of WLSS. 
8:26 - 8:28 am

\title{
Limb and Extremity Undergrowth in Perineuriomas: An Under-recognized Association
}

\author{
Courtney Pendleton MD; Karina Lenartowicz BS; Robert J. Spinner MD
}

Introduction: Perineuriomas are a rare benign hypertrophic nerve lesion, most frequently occurring in young patients. The true incidence may be higher, it is often underdiagnosed. Patients with perineurioma have been anecdotally found to have upper or lower limb/extremity (hand/foot) undergrowth. However this has not been systematically evaluated.

Methods: Archived electronic records from 1990-2018 from a single institution were reviewed for pathology or radiology reports documenting a diagnosis of perineurioma. This identified 110 patients. We further reviewed patients with a documented limb length or extremity size discrepancy.

Results: In total, 25 patients had either a limb length discrepancy or a hand/foot size discrepancy. Nine patients had only a limb length discrepancy, 4 patients had a hand/foot size discrepancy, and 12 patients had both limb and extremity discrepancy.

Fourteen patients had specific details of limb length discrepancy documented: the average discrepancy was $2.7 \mathrm{~cm}$, ranging from $0.5-8.0 \mathrm{~cm}$. Six patients with foot size discrepancy had specifics documented: 4 patients had shoe size discrepancies recorded (average 1.25 sizes); 3 patients had length in cm documented (average $1.67 \mathrm{~cm})$. No patients with hand size discrepancy had specific details recorded.

Patients with limb and extremity undergrowth were significantly younger at age of diagnosis than patients without undergrowth: 12.12 years $v .28 .84$ years $(p<0.0001)$

Conclusion: Limb and extremity undergrowth is likely underreported in patients with perineuriomas. Within our series, patients with documented limb length discrepancy and hand/foot size discrepancy were likely to be significantly younger at diagnosis than patients without recorded undergrowth. The presence of limb and extremity size discrepancy supports the theory that perineurioma may be a congenital lesion. 
8:28 - 8:30 am

\section{Three Fraction Stereotactic Body Radiation Therapy Results in Similar Functional Outcomes as Single Dose Therapy}

Vikram Mehta MPH, MD; Christine Park BA; Luis Ramirez MPH; Elizabeth P. Howell BS; Tara Dalton; John Kirkpatrick; Muhammad M. Abd-El-Barr MD, PhD; Isaac O. Karikari MD; Jordan Torok; Scott Floyd ; CR Goodwin

Introduction: Performance status is an important determinant of the available treatment options for patients diagnosed with spinal metastasis. Spine stereotactic body radiation therapy (SBRT) is an emerging treatment option designed to improve outcomes such as local tumor control compared to conventional radiotherapy.

Methods: A single-institution retrospective study was performed of 156 adult patients with spinal metastases treated with single- or 3-fraction SBRT from 2008 to 2019. Demographics and baseline characteristics, radiographic data, and minimum 3-month follow-up post-treatment outcomes including patient-reported pain scores, tumor progression, performance status, and morphine equivalent dosing were recorded.

Results: Of the 156 patients included in the study, 70 (44.9\%) underwent single-fraction SBRT (median total dose $1700 \mathrm{cGy}$ ) and 86 (55.1\%) underwent 3-fraction SBRT (median total dose $700 \mathrm{cGy}$ ). At baseline, a greater number of patients reported weakness in the 3-fraction group compared to those in the single-dose fraction group $(p<0.05)$. More patients in the 3-fraction cohort were also undergoing treatments other than radiotherapy $(p<0.05)$, had prior resection to the site of SBRT $(p<0.001)$, and had worse modified Rankin Score $(m R S)(p<0.01)$ and Karnofsky Performance Status (KPS) scores $(p<0.01)$ compared to those in the single-dose cohort. At 3-month follow-up, patients treated with single-dose and 3-fraction SBRT demonstrated similar improvements in $\mathrm{mRS}$ scores ( $p=0.0008$ and $p<0.0001$, respectively) with no significant difference in KPS scores.

Conclusion: Patients in the 3-fraction SBRT group showed similar functional outcomes as those in the singledose group. Future studies should consider the effect of prior treatment to the site of SBRT and other metastatic spine tumor assessment metrics such as the Tokuhashi score, Bilsky grade, and spinal instabilityneoplasticscore to differentiate the factors that affect the decision for fractionated SBRT. 
8:30 - 8:32 am

\section{Fractionated Stereotactic Body Radiation Therapy Results in Improved Pain Outcomes For Patients with Spinal Neoplasms}

Vikram Mehta MPH, MD; Christine Park BA; Luis Ramirez; Elizabeth P. Howell BS; Meghan Price; John Kirkpatrick; Muhammad M. Abd-El-Barr MD, PhD; Isaac O. Karikari MD; Jordan Torok; CR Goodwin

Introduction: Spinal stereotactic body radiation therapy (SBRT) offers a non-invasive treatment modality for the control of spinal neoplasms. High radiation doses can cause adverse outcomes, fractionated delivery of SBRT may improve its safety profile while maintaining its efficacy.

Methods: A single-institution retrospective study was performed of 156 adult patients with spinal metastases treated with single- or 3-fraction SBRT from 2008 to 2019. Demographics and baseline characteristics, radiographic data, and minimum 3-month follow-up post-treatment outcomes including patient-reported pain scores, tumor progression, performance status, and morphine equivalent dosing were recorded.

Results: Of the 156 patients included in the study, 70 (44.9\%) underwent single fraction SBRT and 86 (55.1\%) underwent 3-fraction SBRT. At baseline, higher proportion of patients in the 3-fraction group had previous nonSBRT radiotherapy $(p<0.0001)$, had prior resection to the site of SBRT $(p<0.001)$, presented with greater severity of pain $(p<0.05)$, and exercised greater pain medication use on average $(p<0.001)$ compared to that in the single-dose fraction cohort. At 3-month follow-up, although there was no significant difference in the mean pain medication usage both between and within the two groups, the 3-fraction cohort experienced a greater frequency of improved pain outcomes compared to the single-fraction group $(p<0.05)$.

Conclusion: A greater frequency of patients who received fractionated delivery of spinal SBRT achieved significant pain relief compared to those treated with single-fraction. Future work is needed to further establish the relationship between fractionation schedule and clinical outcomes including consideration of primary tumor type and effect on health-related quality of life metrics. 
8:32 - 8:34 am

\section{Separation Surgery and Adjuvant Stereotactic Radiation for Renal Cell Carcinoma Spinal Metastases: Analysis of 91 Cases}

Ibrahim Hussain; Lily McLaughlin; Ilya Laufer MD; Mark H. Bilsky MD; Ori Barzilai MD

Introduction: The management of renal cell carcinoma (RCC) spinal metastases is controversial in regards to extent of resection and radiation dosing.

Methods: A retrospective study of a prospectively-collected cohort from 2003-2017 with surgically-treated, pathology-proven active RCC spinal metastases.

Results: 91 patients were included (mean age 62.2 years). $89 \%$ involved the thoracolumbar spine. Median levels decompressed and construct length were 3 and 5, respectively. Average operative time, blood loss, and length of stay were 176 minutes, $1.3 \mathrm{~L}$, and 5.9 days, respectively. The average dose, fractions, and time from surgery for radiation were $26.9 \mathrm{~Gy}, 2.9$ fractions, and 30.5 days. The complication rate was $17.6 \%$ ( $N=4$ wound-related, $\mathrm{N}=7$ hardware-related, $\mathrm{N}=3$ radiation-related. There was an average 0.13 -point improvement in ECOG status at 1 year ( $N=45)$. Progression free survival (PFS) was $89 \%$ with reirradiation required an average of 1.9 years postoperatively. Reoperation rate for recurrence after failing radiation was $7.7 \%$ a median of 422 days from initial surgery. For deceased patients, median survival was 352 days (40-2,315 days). For living patients, median follow-up was 1,163 days (58-2,624 days).

Conclusion: We present the largest series to date of outcomes for patients treated with Hybrid Therapy for RCC spinal metastases. Separation surgery with adjuvant single-fraction or hypofractionated radiation is safe and effective with stable ECOG status at 1 year, 89\% PFS rate, and 352-day median survival in deceased patients. 
8:34 - 8:36 am

\section{Surgical Treatment of Cervicothoracic Junction Tumors}

Ibrahim Hussain; Joseph Carnevale MD; Jemma Villavieja; Lily McLaughlin; Ori Barzilai MD; Ilya Laufer MD; Mark H. Bilsky MD

Introduction: Stabilization of the cervicothoracic junction (CTJ) in the setting of tumor excision is associated with region-specific challenges.

Methods: A retrospective study of a prospectively-collected cohort from 2011-2018 of patients undergoing decompression and instrumented stabilization across the CTJ for spinal tumors.

Results: 70 patients were included (mean age 60.8 years). The most common primary malignancies were NSCLC $(\mathrm{N}=13)$, renal $(\mathrm{N}=10)$, and breast $(\mathrm{N}=7)$. Median levels decompressed and construct length were 3 and 7.5 , respectively. Average operative time, blood loss, and length of stay were 175 minutes, $557.5 \mathrm{~mL}$, and 7.5 days, respectively. 50 patients received adjuvant radiation, with median dose, fractions, and time from surgery being $27 \mathrm{~Gy}, 3$ fractions, and 20 days, respectively. All patents had lateral mass and pedicle screw instrumentation. 41 patients had tapered $(4.0 / 5.5$ or $3.5 / 5.5 \mathrm{~mm})$ rods, 28 had uniform diameter $(3.5 \mathrm{or} 4.0 \mathrm{~mm})$ rods, 1 had both. 10 patients required anterior reconstruction with PMMA. The complication rate was $15.7 \%$ $(\mathrm{N}=5$ wound, $\mathrm{N}=4$ hardware, $\mathrm{N}=1$ both, $\mathrm{N}=1$ sepsis/pneumonia). For the 5 patients with hardware failure, 4 had tapered rods, and all had cervical screw pullout (of these 1 also had rod/screw fracture). The average time to hardware failure was 134.8 days. For deceased patients $(\mathrm{N}=48)$, median survival was $155.5(8-1,504)$ days. For living patients, median follow-up was 383.3 (59-2,353) days.

Conclusion: Instrumented stabilization across the CTJ for spinal tumor reconstruction can be accomplished using tapered or uniform diameter rods with good outcomes. Tapered rods demonstrate a 2.7-fold increase in hardware failure compared with uniform diameter rods. 
8:36 - 8:38 am

\section{The Change in Chronic Opioid Use Rates After Lumbar Decompression Surgery Following a State- Level Prescribing Reform}

Robert Winkelman BS; Pavitra Ravishankar; Edward Barksdale; Michael Kavanagh; Joseph E. Tanenbaum PhD; Dominic Pelle MD; Edward C. Benzel MD; Thomas E. Mroz MD; Michael P. Steinmetz MD

Introduction: On August 31st 2017, the state of Ohio implemented legislation limiting opioid prescriptions for acute pain. Although state-level prescribing reforms have been shown to reduce overall opioids prescribed following discharge from surgery, little is known about how these reforms may impact the prevalence of chronic opioid use following elective surgery.

Methods: This study is a retrospective review of the postoperative opioid prescribing practices at a single healthcare system for 1031 patients undergoing lumbar laminectomy between 9/1/20168/31/2018. Patient demographics and outpatient opioid use three months prior and twelve months following surgery were obtained from the electronic medical record. Chronic opioid use following surgery was assessed at three, six, and twelve months of follow-up between the two groups.

Results: Ninety-seven percent of patients received an outpatient opioid prescription at discharge. Three, six, and twelve-month follow-up data were available for $88 \%, 83 \%, 73 \%$ of patients, respectively. The prevalence of chronic use in the year before and after the reform was $33 \%$ and $29 \%$ at three months $(-3.5 \%, 95 \% \mathrm{Cl}$ : $10.6 \%$ to $+3.1 \%), 26 \%$ and $23 \%$ at six months $(-3.4 \%, 95 \% \mathrm{Cl}:-11.2 \%$ to $+5.2 \%)$, and $12 \%$ and $10 \%$ at twelve months (-2.2\%, $95 \% \mathrm{Cl}:-8.2 \%$ to $3.1 \%)$, respectively.

Conclusion: The prevalence of chronic opioid use after elective lumbar laminectomy surgery was observed to decrease at three, six, and twelve months in the year following the state-level reform. However, none of the decreases in prevalence were found to be statistically significant. Given the relatively small decreases observed in this study, studies of larger patient populations are likely needed in order to detect a change in the prevalence of chronic opioid use following state-level opioid prescribing reforms. 
8:38 - 8:40 am

\section{Opiate Epidemic Consequences in Kentucky: Trends of Spinal Infections from 2014 to 2019 at a Tertiary Referral Center}

Candice Nguyen BS, RN; Meena Thatikunta; Stephen Furmanek; Luke H. Pearson ; Mayur Sharma MD, MPH; Forest Arnold; Thomas J. Altstadt MD; Maxwell Boakye MD; Emily P. Sieg MD

Introduction: Kentucky ranks 7th in opioid abuse related deaths nationally. IV drug abuse is a known risk factor for spinal infections (SI). Rapid increases in the rates of SI over the last decade have been noted at several neurosurgical centers but Midwestern/Appalachian populations are not well described.

Methods: Retrospective review of patients carrying diagnoses of spinal epidural abscess (SEA), osteomyelitis (OM), and/or discitis (DS) treated at University of Louisville Hospital from 2014 to 2019 was performed. Demographic, infectious, surgical and charge data were analyzed annually and between IV drug abusers (IVDA) and non-IV drug abusers.

Results: A cohort of 76 patients was included. Annual increase in incidence of SI was seen. $49 \%$ of patients were found to be IVDA only and 13\% were opiate dependent and IVDA. Incidence of IVDA increased sharply between 2016 to 2017 (36\% to 56\%). 52\% had operative pathology at first admission. Increasing complexity of surgery is noted with readmission. $41 \%$ of patients were readmitted during the study period and IVDA was an independent risk factor. Charge was highest in those with SEA or Medicare. Readmission charges mirror initial admission charges.

Conclusion: Increasing incidence of spinal infections at our center mirror national shifts in the opiate epidemic with a sharp increase between 2016 and 2017. >50\% require surgery and are prone to reoperation. High charges are maintained at each readmission. 
8:40 - 8:42 am

\section{Ketorolac Significantly Reduces Opioid Use After MIS Thoracolumbar Fusions: Results from a Randomized Controlled Trial}

Chad F. Claus DO; Evan J. Lytle DO; Doris Tong; Diana Sigler R.Ph; Matthew Bahoura BA; Lucas Garmo; Michael H. Lawless BS, DO; Dejan Slavnic DO; Jacob Jasinski DO; Robert W. McCabe DO; Ascher Kaufmann; Gustavo Anton; Karl Kado; Daniel A. Carr DO; Prashant S. Kelkar DO; Clifford M. Houseman DO; Peter L. Bono DO; Boyd Richards; Teck M. Soo

Introduction: Postoperative pain control following spinal fusion remains difficult and limited to opioids. Opioid use remains an important public health concern. Ketorolacs immediate impact on safely reducing opioid consumption while demonstrating equivalent trends in fusion rates is becoming evident.

Methods: A multi-hospital, prospective, double-blinded, randomized placebo-controlled trial of patients undergoing 1 to 3-level MIS thoracolumbar TLIF is ongoing. Patients were randomized to receive a 48-hour scheduled treatment of either intravenous Ketorolac or saline in addition to a standardized pain regimen. Fusion is the primary outcome. Secondary outcomes include postoperative opioid use demonstrated as morphine equivalence, pain scores, length of stay, as well as QoL outcomes. Univariate analysis was performed. P-value 0.05 was considered significant.

Results: One hundred and eighty-two patients were analyzed per protocol. Patient characteristics and operative data were comparable between the groups. There was a significant reduction in total/48-hour opioid consumption $(p=<0.0001)$ and length of stay $(p=0.0006)$ for the Ketorolac group while demonstrating equivalent postoperative pain scores $(p=0.45)$. There was no significant difference in rates of perioperative complications. One hundred and sixty-two levels at 6-months and 90 levels at 1-year were evaluated for fusion. Ketorolac had no significant effect on fusion rates at 6-months $(p=0.70)$ or 1 -year $(p=0.39)$.

Conclusion: Ketorolac safely demonstrated a significant reduction in postoperative opioid consumption and length of stay. Ketorolac continues to demonstrate no impact on rates of pseudoarthrosis. 
Spine Summit Meeting Abstracts, May 2021

8:42 - 8:44 am

\section{Surgical Treatment of Intradural Extramedullary Nerve Sheath Tumors with Neurofibromatosis 1 (NF1) at a Cancer Center}

Kimberly B. Hoang MD; John Slopis; Claudio E. Tatsui MD; lan E. McCutcheon MD

Introduction: Multiple symptomatic intradural extramedullary lesions are a major cause of myelopathy in NF1 and schwannomatosis patients, and are surgically challenging. Few large series areavailable.

Methods: We systematically reviewed NF1 patients with symptomatic spinal lesions from 1993 to 2018. Univariate and multivariate statistical analyses were performed. We describe the inside-out, "intraduralto-extradural" resection technique for maximal safe resection under intraoperative monitoring while minimizing cord manipulation and surgical exposure.

Results: Forty-eight patients underwent 67 surgeries and 53 unique sites of disease. Presenting symptoms were primarily pain $(66.3 \%)$, weakness $(58.5 \%)$, and numbness $(41.5 \%)$. The median number of tumors resected was two (range 1-8) with a recurrence rate of $20.8 \%$; median duration to recurrence was 52 months (range 6-107 months). Extent of resection (EoR) was gross total in 41.5\%, near total in 18.9\%, and subtotal in $30.9 \%$. Four patients developed postoperative spinal deformity at a median time of 18.5 (range 7-48) months. Detailed motor scores at multiple time points demonstrated $16.98 \%$ improved immediately after surgery; increased to $39.02 \%$ improved at last follow up (Figure 1). On univariate analysis, EoR was significantly correlated only with the number of tumors resected $(p=0.03)$ and iatrogenic deformity with the number of levels operated $(p=0.006)$. Multivariate analysis of recurrence indicated diagnosis (neurofibromatosis vs. schwanommatosis) and presenting symptoms were significant predictors $[p=0.02 ; p=0.03$. Multivariate analysis of functional improvement showed the presence of post-operative complication(s) and the immediate post-op motor score were significant predictors $[p=0.03 ; p=0.008]$.

Conclusion: This is the largest series characterizing this patient population. Resection of symptomatic, multilevel intradural tumors in NF1 patients is feasible with good extent of resection and improvement or preservation of neurologic function. 
Spine Summit Meeting Abstracts, May 2021

8:44 - 8:46 am

\section{The "Hit Rate" For Determining Malignancy in Percutaneous Biopsy of Peripheral Nerve Tumors of Uncertain Behavior}

Courtney Pendleton MD; Robert J. Spinner MD

Introduction: The diagnostic workup of peripheral nerve tumors is complicated. Biopsy of benign nerve sheath tumors has not been recommended due to its potential for complications including nondiagnostic or misdiagnosed tissue, neurologic compromise or more difficult tumor resection from scarring. In patients with peripheral nerve tumors of uncertain behavior, tissue diagnosis confirming a malignancy may significantly alter treatment plans and prognosis.

Methods: The notes of a single surgeon were reviewed from 2000-2018. Inclusion criteria were presence of a peripheral nerve tumor, biopsy obtained at our institution, and a pathology report available for review. When patients underwent resection of their tumor, those pathology reports were reviewed, and that diagnosis was considered as the "final diagnosis." Patients with divergent biopsy and resection pathology were considered to be misdiagnosed. The "hit rate" was determined to be the percentage of patients sent for biopsy who had a malignancy on their final pathology.

Results: In total, 159 patients met our inclusion criteria. 104 (65.4\%) were referred for biopsy after neurosurgical consultation. 55 (34.59\%) were referred for biopsy by a non-neurosurgeon.

The neurosurgical group included 76 patients with tumors of uncertain behavior (73\%); the non-neurosurgical group included 26 patients $(42.3 \%)$ ( $p=0.0002)$. The neurosurgical group had a hit rate of $44.7 \%$, while the non-neurosurgical group was $7.7 \%(p=0.0007)$.

The neurosurgical group included 16 MPNST, 7 other sarcomas (synovial sarcoma, Ewing sarcoma, liposarcoma), 8 metastatic malignancies, and 1 neuroblastoma. The non-neurosurgical group included 1 sarcoma and 1 metastatic adenocarcinoma.

Conclusion: Increased familiarity with clinicoradiological findings associated with MPNST, and experience differentiating malignant, benign, and uncertain tumors may allow neurosurgeons, particularly peripheral nervefocused neurosurgeons, to better identify patients who will benefit from a percutaneous biopsy. 
8:46 - 8:48 am

\title{
Predictive Modeling of Long-Term Postoperative Opioid Use after Spine Surgery
}

\author{
Yi Zhang; Parastou Fatemi; John K. Ratliff MD, FACS
}

Introduction: No guideline exists for identifying or effectively reducing outpatient postoperative opioid use in spine patients.

Methods: In this retrospective cohort study, patients who were newly diagnosed with back pain between 2008 and 2015, were opioid-naive 6 months prior to diagnosis, and underwent spine surgery within one year after diagnosis were enrolled. We required at least 12 months of postoperative follow-up and at least one opioid prescription filled within the 30 days following surgery. Long-term opioid use was defined as filling $\geq 180$ days of opioids within one year after surgery. Using demographic variables, comorbidities, preoperative opioid use, and 30-day postoperative opioid prescriptions, we generated and evaluated several models, including stepwise logistic regression, least absolute shrinkage and selection operator (LASSO), support vector machine, random forest, stochastic gradient boosting, and convolutional neural network.

Results: 903 of 19,317 patients met criteria for long-term opioid use. The full logistic regression model demonstrated the highest sensitivity at $79.2 \%$, while stepwise logistic regression demonstrated the highest area under the curve (AUC) at 0.845 . The three strongest predictors of long-term opioid use were number of 30-day postoperative opioid prescriptions (OR 2.28; 95\% Cl 1.98-2.64), preoperative opioid prescription pattern (OR 1.97; 95\% Cl 1.75-2.23), and number of dosage increases between postoperative day 15 to 30 (OR 1.95, 95\% Cl 1.69-2.26).

Conclusion: We evaluate several predictive models for postoperative long-term opioid use in a large cohort of spine patients, which may be used to identify patients at high risk of long-term opioid use based on preoperative risk factors and opioid prescription pattern in the first 30 days after surgery. 
8:48 - 8:50 am

\section{c on Opioid Requirement and Pain Control in Patients Undergoing Lumbar Fusions}

Alan T. Villavicencio MD; Sharad Rajpal MD; Ewell L. Nelson MD; Steven L. Hobbs PhD; Christopher Zielenski PharmD; Kara D. Beasley; Vinod Kantha BA; Sigita Burneikiene MD

Introduction: Preventive multimodal analgesia (PMA) addresses multiple sources and pathways of acute and chronic pain by interfering with peripheral and central sensitization and should provide a way to achieve safer and more effective pain management with reduced opioid medication use.

Methods: A prospective observational study with a historical reference group was performed. The study compared postoperative opioid requirement and analgesic effect in a total of 103 patients undergoing elective, one- or two-level transforaminal lumbar interbody fusion (TLIF) surgeries for symptomatic lumbar degenerative disc disease. The PMA patient group included 52 consecutive patients who received $1000 \mathrm{mg}$ of acetaminophen, $300-900 \mathrm{mg}$ of gabapentin, and $200-400 \mathrm{mg}$ of celecoxib one hour before the procedure. The reference group included 51 patients who received $15 \mathrm{mg}$ of morphine-equivalent dose (MED) preoperatively.

Multiple linear regression was used to evaluate the influence of PMA on pain, MED and acetaminophen use over 4 postoperative days (PODs), while controlling for all variables likely to influence these outcomes, including age, gender, baseline opioid use, duration of surgery, post-operative intrathecal morphine use and administration of muscle relaxants and anticonvulsants.

Results: The differences in opioid requirement and postoperative pain scores were statistically significant on all four postoperative days. The effect size varied from -0.54 to -0.99 for the postoperative opioid requirement and from -0.59 to -1.16 for postoperative pain indicating that these measures were reduced by about $1 / 2$ to 1 standard deviation in the PMA patient group.

Conclusion: PMA is a highly effective method for postoperative pain management in patients undergoing lumbar fusion surgeries, which not only improves pain control, but also reduces opioid requirement. 
8:50 - 8:52 am

\section{Trends in Spine Surgery Training during Neurological and Orthopedic Surgery Residency: A Ten-Year Analysis of ACGME Data}

Martin H. Pham MD; Arvin R. Wali BA; Andre M. Jakoi; Lawrence Lenke MD

Introduction: Spine surgery training in the United States currently involves residency training in neurological or orthopedic surgery.

Methods: The Accreditation Council of Graduate Medical Education resident case logs for both orthopedic surgery and neurological surgery were reviewed for exposure to spine surgery procedures for the graduating years of 2009-2018.

Results: The average number of spine surgery procedures performed during that 10 -year period was 433.8 for neurosurgery residents and 119.5 orthopedic surgery residents $(p<0.01)$. From 2009 to 2018, neurosurgery residents saw an increase of $26.5 \%$ in spine surgery procedures from 389.6 to 492.9 procedures whereas orthopedic surgery residents saw a decrease of $41.3 \%$ from 141.1 to 82.8 procedures. The 10 -year average percentage of total spine procedures of all total surgical cases was $33.5 \%$ for neurosurgery residents compared to $6.2 \%$ for orthopedic surgery residents $(\mathrm{p}<0.01)$. This percentage decreased both for neurosurgery residents (35.8\% in 2009 to $31.3 \%$ in 2018 ) and orthopedic surgery residents $(7.2 \%$ in 2009 to $4.9 \%$ in 2018$)$. Neurosurgical residents performed on average 3.6 times more total spine procedures than orthopedic surgery residents, a number that increased from 2.8 fold in 2009 to 6.0 fold in 2018.

Conclusion: Case volume of spine surgery procedures vary greatly with higher rates for neurological and lower for orthopedic surgery residencies, with an enlarging increasing discrepancy over time. 
8:52 - 8:54 am

Race is a Predictor of All-Cause Mortality in Patients Diagnosed with a Primary Spine Tumor

Aladine A. Elsamadicy BE; Isaac G. Freedman BPhil, MPH; Andrew B. Koo ; Wyatt David; Adam Kundishora MD; Ramana Gorrepati MD; Gregory A. Kuzmik MD; Luis Kolb MD; Maxwell Laurans; Khalid M. Abbed MD

Introduction: The influence that racial disparities have on mortality rates in spine tumor patients is relatively unknown.

Methods: The Surveillance, Epidemiology, and End Results (SEER) Registry was used to identify all patients with a coded spinal tumorfrom 1973 through 2016. Racial groups (Black vs. White) were balanced using propensity-score matching using a non-parsimonious 1:1 nearest neighbor matching algorithm.

Results: A total 7,498 patients were identified with a primary tumor of the spine (Black:648 vs.

White:6,850). After PS-matching, both groups were matched across all demographic, tumor, and treatment variables. Black patients with primary spinal tumors were more likely to die of all causes than were White patients in both the non-PS-matched (HR: 1.26, 95\% Cl: 1.04-1.51,P=0.01) and PS-matched cohorts $(H R$ : 1.64, 95\% Cl: 1.28-2.11, $P<0.0001)$. Patients who received RT were also more likely to die of all causes than were patients who did not in both the non-PS-matched (HR: 2.78, 95\% Cl: 2.43-3.18,P<0.0001) and PSmatched cohorts $(H R: 3.15,95 \% \mathrm{Cl}: 2.36-4.19, P<0.0001)$. On multivariate $\mathrm{CPH}$ regression analysis age at diagnosis (HR: $1.03,95 \% \mathrm{Cl}: 1.02-1.05, P<0.0001)$, race (HR: $1.82,95 \% \mathrm{Cl}: 1.22-2.74, P=0.004)$, and receipt of RT (HR:2.62, 95\% Cl: 1.56-4.37,P=0.0002) were all significantly associated with all-cause mortality, when controlling for other demographic, tumor, and treatment variables.

Conclusion: Our study demonstrates a population-based estimates of the prognosis for patient with spine tumors and suggests that racial disparities may influence all-cause mortality. 
8:54 - 8:56 am

\section{Survival and Fusion Outcomes after Surgery for Spinal Metastatic Disease}

Timothy J. Yee; Yamaan S. Saadeh MD; Michael J Strong MD, PhD; Clay M. Elswick MD; Ayobami Ward MD; Paul Park MD; Mark E. Oppenlander MD; Nicholas J. Szerlip MD, FACS

Introduction: Decompression and fusion is integral to the multidisciplinary approach to patients with symptomatic spinal metastatic disease. Bony fusion is typically sought despite procedural morbidity and historically poor prognosis.

Methods: We performed a retrospective cohort study of adult patients undergoing decompression and fusion for spinal metastases at a single institution. Patients were followed clinically until death or loss to follow-up. Extent of fusion was assessed with CT scans at 6-month intervals from 6 months to 24 months postoperatively barring death or loss to follow-up. Cumulative incidence estimates for fusion and hardware failure accounted for the competing risk of death.

Results: One hundred fifty-seven consecutive patients were identified. Mean age \pm SD was $62.4 \pm 10.7$ years, $61.2 \%$ were male, $98.7 \%$ received allograft and/or autograft, and $91.7 \%$ received postoperative radiation. Kaplan-Meier estimate of median survival was 10.3 (IQR 3.6-32.7) months. Proportions with any fusion and with complete fusion were $23.6 \%$, and $6.4 \%$. At the longest observed time to any fusion of 29.3 months, estimated cumulative incidence of any fusion was $30.5 \%(95 \% \mathrm{Cl} 22.6-38.8 \%)$. At the longest observed time to complete fusion of 23.3 months, the estimated cumulative incidence of complete fusion was $8.7 \%(95 \% \mathrm{Cl} 4.4-$ $14.8 \%$ ). Of patients surviving 6 months and 12 months, complete fusion was observed in $12.9 \%$ and $16.7 \%$. Risk of hardware failure requiring reoperation was 3.2\%. At the longest observed time to hardware failure of 44.3 months, the estimated cumulative incidence was $4.6 \%$ (95\% Cl 1.6-10.2\%). Using a multivariate Fine and Gray proportional subdistribution hazard model, increasing age independently predicted hardware failure (HR $1.2, p=0.0058)$.

Conclusion: Low rates of complete fusion and hardware failure were observed due to the high competing risk of death. Efforts to redirect the traditional emphasis on bony fusion may be warranted. 
8:56 - 8:58 am

\section{Targeting Histone and DNA Methylation as a Therapeutic Strategy for Chordoma}

Sakibul Huq; Jayanidhi Kedda; Tianna Zhao; Riccardo Serra MD; Andy Ding; Manuel Morales; Jeffrey Ehresman; Henry Brem MD; Gary L. Gallia MD; Daniel M. Sciubba MD; Betty Tyler

Introduction: The epigenetic landscape of chordoma has recently been described as involving a distinct histone code and DNA methylation profile, both of which may contribute to chordoma tumorigenesis. Strategies targeting epigenetic changes using small molecule inhibitors of histone and DNA methylation have demonstrated therapeutic promise in multiple cancers. However, these approaches have not been explored in chordoma.

Methods: Multiple in vitro assays were performed using DZNeP and decitabine in sacral (U-CH1) and clival (UM-Chor1) chordoma cell lines. Cell growth was evaluated via Cell Counting Kit 8 assay. Clonogenic potential was determined via colony formation assays. Cell death was assessed via flow cytometry with AnnexinV (AnnV) and Propidium lodide (PI) staining. Proliferation assays were performed to quantify cellular growth over three time points.

Results: DZNep inhibited cell growth with an IC50 of 1.3uM in both U-CH1 and UM-Chor1 cells. DZNep diminished clonogenic potential in both cell lines, including complete elimination of clonogenic potential in UMChor1 cells. DZNep promoted apoptosis in UM-Chor1 cells, indicated by an increase in AnnV+/PI- and AnnV+/PI+ cells. The combination of DZNeP and decitabine led to decreased cell growth compared to either drug alone in UM-Chor1 cells.

Conclusion: Global inhibition of histone methylation decreased cell growth, proliferation, and clonogenic potential in addition to increasing cell death in chordoma cell lines. The combination of inhibiting both histone and DNA methylation led to more potent inhibition of cell growth than either strategy alone. This combination therapy warrants further investigation in chordoma in vitro and in vivo. Epigenetic modulation may represent a promising new therapeutic strategy for chordoma. 
8:58 - 9:00 am

\section{Impact of Frailty on Outcomes following Elective Spine Surgery: A Prospective Cohort Analysis of 668} Patients

Nitin Agarwal MD; Ezequiel Goldschmidt MD, PhD; Tavis Taylor BA; Souvik Roy; Stephanie Dunn PhD; Andrew Bilderback MS; Robert M. Friedlander MD; Adam S. Kanter MD; David O. Okonkwo MD, PhD; Peter Gerszten; David Hamilton; Daniel Hall MD

Introduction: With an aging population, elderly patients with multiple comorbidities are more frequently undergoing surgical intervention and may be at an increased risk for perioperative complications. Objective measurement of a frailty score may predict the incidence of postoperative adverse events.

Methods: As part of a system-wide quality improvement initiative, frailty assessment was added to the routine assessment of patients considering spine surgery beginning in July 2016 . Frailty was assessed with the Risk Analysis Index (RAI), and patients were categorized as non-frail (RAI 0-29) or pre-frail/frail (RAI $\left.{ }^{3} 30\right)$. Blood loss, length of stay (LOS), thromboembolic events, surgical site infections, readmissions and mortality were taken as outcome variables. Comparisons between non-frail and pre-frail/frail patients were analyzed by Pearson's chi-squared tests for categorical data or by Wilcoxon rank sum tests for continuous data.

Results: From August 2016 through September 2018, 668 patients (age of $59.5 \pm 13.3$ years) had a preoperative RAI score recorded and underwent elective spine surgery. As expected, pre-frail and frail patients suffered comparatively higher rates of mortality at 90 -days $(1.9 \%$ vs $0.2 \%, p<0.05)$ and one-year $(5.1 \%$ vs $1.2 \%, p<0.01)$ from the procedure date. They also had longer in-hospital LOS (3.9 days \pm 3.6 vs. 3.1 days \pm $2.8, p<0.001)$, shorter operative time $(149 \pm 84 \mathrm{~min}$ vs $163 \pm 84 \mathrm{~min}, \mathrm{p}<0.05)$, and higher rates of 60 day $(14.6 \%$ vs $8.2 \%, p<0.05)$ and 90 -day $(15.8 \%$ vs $9.8 \%, p<0.05)$ re-admissions.

Conclusion: The RAl effectively risk stratifies spine patients and may inform perioperative decision making, including the use of preoperative interventions which may mitigate these negative outcomes. 
9:00 - 9:02 am

\section{Outcomes and Hypercoagulable Complications Associated with Tranexamic Acid During Thoracolumbar Three Column Osteotomies}

Darryl Lau; Alexander F. Haddad BS; Rushikesh S. Joshi BS; Vedat Deviren MD; Christopher P. Ames MD

Introduction: The use of three column osteotomies (3CO) for correction of fixed thoracolumbar deformities are associated with high complications and large volume blood loss.

Methods: A retrospective review of consecutive ASD patients from 2006 to 2019 who underwent a posterior thoracolumbar $3 \mathrm{CO}$ was performed. Hypercoagulable complications and various outcomes between patients who received TXA and who did not were compared. Multivariate regression was used.

Results: 365 patients were included: 182 TXA vs. 183 without TXA. Mean age was 64.7 years with $60.4 \%$ female patients. Patients without TXA had higher preoperative history of DVT/PE $(7.7 \%$ vs. $2.8 \%, p=0.029)$.

There were similar rates of overall $(28.4 \%$ vs. $30.8 \%, p=0.622)$ and hypercoagulable $(3.8 \%$ vs. $2.2 \%, p=0.274)$ complication between the groups. There was no significant difference in blood loss (1923.3 vs. $2032.1 \mathrm{~mL}$, $p=0.436)$. Patients with TXA had significantly shorter operative time $(294.8$ vs. 321.7 minutes, $p<0.001)$ and use of TXA was independently associated with shorter operative time $(p<0.001)$. Operative time was inversely dose dependent as the highest dose infusion resulted in the shortest operative time: no TXA (321.7 minutes), 1 $\mathrm{mg} / \mathrm{kg} / \mathrm{hr}$ (292.1 minutes), $2 \mathrm{mg} / \mathrm{kg} / \mathrm{hr}$ (301.8 minutes), and $3 \mathrm{mg} / \mathrm{kg} / \mathrm{hr}$ (288.9 minutes) ( $\mathrm{p}<0.001)$.

Conclusion: Routine use of TXA for thoracolumbar $3 \mathrm{CO}$ is safe and there does not seem to be a higher risk for hypercoagulable complications. In addition, TXA is able to shorten operative duration perhaps facilitating more efficient procedures secondary to decreased bleeding. 
9:02 - 9:04 am

\section{Predictors of Indirect Neural Decompression with Lateral Transpsoas Interbody Fusion}

Corey T. Walker MD; David S. Xu MD; Jakub Godzik MD, MSc; Ifije Ohiorhenuan MD, PhD; Tyler S. Cole MD; James Zhou MD; Jay D. Turner MD, PhD; Juan S. Uribe MD, FAANS

Introduction: Restoration of disc height and alignment in lateral lumbar interbody fusion (LLIF) allows for indirect decompression of the neural elements through ligamentotaxis, foraminal height restoration and correction of listhesis.

Methods: A retrospective analysis of consecutive patients treated with transpsoas LLIF was performed at a single institution. Pre-operative and immediate post-operative MRI was performed to determine the extent of indirect decompression. 3-Dimensional volumetric T2 sequencing was used to determine central canal area (CCA) and qualitative stenosis grading.

Results: 110 levels (66 patients) were analyzed. Across levels treated, there was an $83 \%$ increase in CCA. 17 levels had moderate stenosis pre-operatively, all of which improved to mild or no stenosis with a $130 \%$ increase in CCA. 18 levels had severe stenosis pre-operatively, 4 which remained severe $(51 \%$ increase in CCA), 5 which improved to moderate (207\% increase in CCA) and 9 improving to mild or no stenosis $(246 \%$ increase in CCA). Of 35 levels with moderate or severe stenosis pre-operatively, the patients who failed improve to mild or no stenosis had similar changes in VAS back (4.1 vs 4.9, $p=0.37$ ) but less improvement in VAS leg (2.9 vs 4.9, p=0.04) compared to those who did. Levels treated for spondylolisthesis (36 levels) and adjacent segment disease (11 levels) had significant greater increases in canal area compared to those treated for disc degeneration (46 levels) and deformity (16 levels) (119\% vs $103 \%$ vs $62 \%$ vs $51 \%$, p 0.05$)$. Pre-operative anterior and posterior disc height were correlated with improvements in change in canal area $(p=0.01, p=0.03$, respectively).

Conclusion: LLIF results in significant increases in CCA, with patients treated for spondylolisthesis and adjacent segment disease responding best to indirect decompression. Patients with persistent stenosis on post-operative MRI have less improvement in VAS leg scores. 
9:04 - 9:06 AM

\section{A Retrospective Analysis of High Opioid Use Patients Undergoing a Preoperative Pain Program Prior to Spine Surgery}

Karl Wittnebel; Lindsey Ross BS, MD; Jeanne Black MBA, PhD; Keith Siegel; Terrence T. Kim MD; Eli M. Baron MD; Alexander Tuchman MD; Tiffany Perry; Mark Vrahas; J. Patrick Johnson; Sang Do Kim

Introduction: Previous studies have shown that after spine surgery, chronic opioid users have worse patient outcomes, increase their long-term opioid use and spend more healthcare dollars (Devin, Lee, Wick, McAnally).

The Preoperative Pain Program (PPP) is a comprehensive treatment program aimed at optimizing high opioid use patients undergoing elective spine surgery.

Methods: This is a retrospective data analysis of High opioid users, 80 MED who were enrolled in a program with a multidisciplinary team that reduced opioid consumption at target rate of $10 \%$ weekly. The primary outcomes include 30-day re-admissions/ ER visits, and LOS. Secondary outcomes include complication rate, 90-day re-operation rate and discharge disposition.

Results: 330 patients underwent treatment in the PPP; thus far 80 have sufficient follow up data. We have identified 1,400 comparison subjects for analysis. Reoperation rates were 6X lower in the PPP group. PROMIS scores were collected before and after weaning, showing no significant increase in pain level despite an average opioid reduction of over $30 \%$.

Conclusion: This study lays the ground work for future research assessing the efficacy of a PPP to improve surgical outcomes for high opioid users and has the potential to change preoperative management of spine patients. The feasibility of decreasing opioid doses before major spine surgery is demonstrated, without undue hardship for patients. We hope that this study will serve as a template for further research on specific opioid weaning targets and lead times associated with maximal benefit, including functional outcomes. 
9:06 - 9:08 am

\title{
Clinical Outcome of Cranio-Cervical Junction Meningioma: A 20-year Single-Institutional Experience
}

\author{
Nida Fatima; Steven D. Chang MD; Antonio Meola
}

Introduction: Craniocervical meningiomas are slow-growing indolent tumors at the foramen magnum junction, which poses surgical challenge due to the critical nearby neurovascular structures.

Methods: A retrospective analysis from a prospectively maintained database from March 1998 to March 2019 at an academic tertiary care hospital was conducted. Depending upon the tumor origin and dural attachment, $66.7 \%$ were craniospinal (CS) and $33.3 \%$ were spinocranial (SC) tumors. Multivariable Cox-Proportional Hazards Regression Model was computed to determine the association of peri-operative factors with the risk of complications postoperatively. All associations were plotted as Hazards Ratio (HR) with 95\% Confidence Interval $(\mathrm{Cl})$.

Results: Statistical analysis of 30 patients $(73.3 \%$ females, median age: 61 years) revealed that the median tumor volume was $7.24 \mathrm{~cm}^{3}$ (range, $1.77-22.4 \mathrm{~cm}^{3}$ ). With a median follow-up of 36 months (range, 0.3-60 months), there was no tumor recurrence. Patients with SC tumor regained almost full daily living activity [(Karnofsky Performance Scale KPS), $88.0 \pm 6.32$ ] compared with CS tumor (KPS, 72.5 \pm 19.9 ) after surgical treatment $(p=0.005)$, and the length of stay in hospital was shorter in patients with SC $(4.60 \pm 2.75$ days $)$ than CS tumors (10.90 \pm 15.8 days) ( $p=0.05)$. Permanent surgical morbidity and mortality was $33.3 \%$ and $10 \%$ respectively. The female gender ( $\mathrm{HR}: 0.78,95 \% \mathrm{Cl}: 0.20-3.05, \mathrm{p}=0.73)$, age $<60$ years ( $\mathrm{HR:} 0.97,95 \% \mathrm{Cl}$ : 0.92-1.03, $\mathrm{p}=0.40$ ), tumor volume $\geq 7.5 \mathrm{~cm}^{3}$ (HR: $\left.1.03,95 \% \mathrm{Cl}: 0.92-1.16, p=0.56\right)$, CS tumors (HR: $2.34,95 \%$ Cl: $0.53-12.34, p=0.23)$, position position to the spinal cord (HR: $0.54,95 \% \mathrm{Cl}: 0.15-1.94, p=0.35)$, Far-Lateral approach (HR: 0.80, 95\% Cl: 0.23-2.78, p=0.73), GTR (1.76, 95\% Cl: 0.45-6.89, p=0.42), and arterial involvement (HR: $1.34,95 \% \mathrm{Cl}: 0.34-5.20, p=0.67)$ were found to associated with the rate of complications, but statistically insignificant.

Conclusion: Compared to CS tumor, patients with SC tumor have better functional outcome and shorter length of stay in the hospital. 
9:08 - 9:10 am

\section{A Naïve Bayes Model to Predict 3-Month Post-Surgical Survival in Metastatic Epidural Spinal Cord Compression Patients}

Vikram Anjur; Chang Hu; Krishnakant Saboo; Ravishankar lyer; Paul M. Arnold MD

Introduction: Nave Bayes classifiers use Bayesian inferencing to estimate a latent variables value based on priors and observations of other variables. When predicting survival, such models can guide a clinicians decision on whether surgical decompression is appropriate for patients with metastatic epidural spinal cord compression (MESCC).

Methods: Data from 142 surgically treated MESCC patients were obtained from the North American AOSpine study, in which 40 patients died within 3 months after surgery and 97 were known to survive. First, preoperative features most predictive of 3-month postoperative survival were identified using backward stepwise feature selection separately in random forest, logistic regression, and linear SVM classifiers. A nave Bayes model was then constructed with these features, assigning each chosen feature to a node. A separate class node was inserted to represent 3-month postoperative survival, and edges were drawn from it to each of the feature nodes. Testing prediction accuracies and area under the receiver operating characteristic curve (AUC) were used to evaluate performance across 10 cross validation trials with randomized $70 \%-30 \%$ trainingtesting splits.

Results: During feature selection, the most frequently used predictors across all classifiers were (i) presence of extraspinal metastasis, (ii) SF-36v2 physical component score, (iii) radiotherapy to MESCC lesion, (iv) severity of comorbidities, and ( $v$ ) gender. The naïve Bayes network built from these 5 features reported $76.98 \% \pm 2.64 \%$ average accuracy and $0.76 \pm 0.04$ average AUC.

Conclusion: We found that with a nave Bayes network, we could predict 3-month postoperative survival in MESCC patients with $76.98 \%$ accuracy using just 5 preoperative features. Further investigation can determine whether modifications suggested by guided structure learning can improve prediction. 
9:10 - 9:12 am

Tracking Neurosurgical Opioid Prescribing Rates in the Medicare Population from 2013-2017

Colin Lamb; Sean N. Neifert BS; Michael Martini; Frank Yuk MD; Jeffrey Gilligan MD; John Caridi

Introduction: While opioid prescribing volume has decreased in the US since 2013, overdoses from opioids have increased, leading to questions about prescribing practices. This study is the first to track the prescribing practices of neurosurgeons from 2013 to 2017 in the Medicare Part D population, which represents approximately $70 \%$ of all Medicare patients.

Methods: Neurosurgeons were identified in the publicly-available Center for Medicaid and Medicare Services (CMS) Part D Prescriber File by National Provider Identifier. Only neurosurgeons with prescribing data in all five of the annual datasets were included $(n=2,046)$. The sum of opioid prescriptions at each year from 20132017 and percent changes from 2013 were calculated. Descriptive statistics analyzed the median number of opioids prescribed and days of opioids prescribed per patient by neurosurgeons at each year.

Results: The sum of opioid prescriptions written by 2,046 neurosurgeons for Medicare Part D patients decreased from 2013 (sum=344,510) by 6,674 (1.93\%), 44,448 (12.9\%), 53,489 (15.53\%), and 75,438 (21.9\%) prescriptions in 2014, 2015, 2016, and 2017, respectively. The annual median number of opioid prescriptions decreased significantly in 2015 ( $M=91, I Q R=51-172, p<0.001), 2016(M=85, I Q R=47-163, p<0.001)$, and 2017 $(M=76, I Q R=39-149, p<0.001)$ compared to $2013(M=102.5, I Q R=54-199)$. Additionally, the median annual number of opioids prescribed per patient decreased significantly in $2014(M=1.95, I Q R=1.57-2.51, p<0.001)$, $2015(M=1.73, I Q R=1.45-2.17, p<0.001), 2016(M=1.68, I Q R=1.38-2.11, p<0.001)$, and $2017(M=1.61$, $I Q R=1.33-2.02, p<0.001)$ from the median in $2013(M=2.04, I Q R=1.65-2.63)$. Similarly, the annual median number of days of opioids supplied per patient significantly decreased in $2014(\mathrm{M}=25.07, \mathrm{IQR}=16.81-36.55$, $\mathrm{p}<0.001), 2015$ ( $\mathrm{M}=23.54, \mathrm{IQR}=16.05-35.65, \mathrm{p}<0.001), 2016(\mathrm{M}=22.96, \mathrm{IQR}=15.24-34.42, \mathrm{p}<0.001)$, and 2017 $(M=21.27, I Q R=14.35-32.11, p<0.001)$ from the median in $2013(M=25.35, I Q R=16.74-38.41)$.

Conclusion: The significant decrease in total and median opioid prescription volume from 2013-2017 suggests that the typical neurosurgeon is both writing fewer opioid prescriptions and prescribing fewer days supply when doing so. Further research will be needed to assess the impact of these changes. 
Spine Summit Meeting Abstracts, May 2021

9:12 - 9:14 am

\section{The Effect of Opioid Dependence on Postoperative Urinary Retention Following Elective Lumbar Fusion Surgery}

Ronan Doherty; Waseem Wahood; Yagiz U. Yolcu MD; Jad Zreik BS; Anshit Goyal MBBS; Mohammed A. Alvi MD; Elizabeth B. Habermann PhD; Mohamad Bydon MD

Introduction: The incidence of opioid dependence is increasing with healthrelated complications impacting both patients and healthcareservices. As one of the complications resulting from opioid use, urinary retentionmayalsobe encounteredfollowingsurgical procedures.

Methods: Patients undergoing elective lumbar fusion were identified in theState Inpatient Databasesbetween 2013-2015. Diagnoses and procedures weredetermined using ICD codes. Exclusion criteria were patients withtumour, trauma and infection (including UTI). Non-opioid andopioid dependent groups were established and matched using 3:1propensity score matching. Patientswith PUR, those with a LOS in the upper quartileand those with a discharge to a location other than homewere flagged. Multivariate logistic regression analysed the impactof opioid use on the above outcomes and Wald chi-squared testsdetermined the top predictors for these outcomes.

Results: Opioid dependence was significant for PUR $(p=0.047)$ and prolonged LOS $(p=0.001)$, but not for nonroutine discharges $(p=0.31)$. Predictors for PUR were deficiency anaemias, hypothyroidism, alcohol abuseand opioid dependence. Predictors for prolonged LOS were electrolyte disorders, deficiency anaemias, US State, coagulopathies, opioid dependency, congestive heart failure, paralysis, liver disease, hypertension, alcohol abuse and drug abuse. Predictors for non-routine discharge were electrolyte disorder, hypertension, hyperthyroidism, paralysis, neurological disorders, renal failure, deficiency anemiasand congestive heart failure.

Conclusion: Opioid dependence has a notable impact on PUR and LOS, but not on NRD. Many other factorswere identified as predictors for these outcomes. This knowledge enables us to identify sourcesof pressure for health services and approach them strategically through increased awareness. 


\title{
9:14 - 9:16 am
}

\section{Risk Factors for Kyphosis and Revision After Surgery for Spinal Metastasis}

\author{
Shashank V. Gandhi; Katherine Wagner; Justin Turpin; Ahmad Latefi DO
}

Introduction: With advances in chemotherapeutics, radiosurgery, and immunotherapies patients with spinal metastasis are living longer than previously described.

Methods: Patients who underwent surgery for spinal metastasis from 2011-2018 were assessed. Patients with incomplete records were excluded. Surgical factors including SINS score, spinal region, stabilization, and anterior column reconstruction were assessed for development vertebral fractures, rod failure, kyphosis, graft subsidence, progression of disease, and need for revision.

Results: 51 patients were assessed. 36 patients had stabilization and 15 had anterior reconstruction. Patients with higher SINS scores were more likely to undergo stabilization $(p=0.005)$ and anterior column reconstruction $(p=0.005)$. The revision rate was $14 \%$ and kyphosis rate was $23.5 \%$. Risk factors for need for revision surgery were anterior graft failure $(p=0.040)$, vertebral body fracture $(p=0.037)$, rod failure $(p=0.001)$, development of kyphosis $(p<0.001)$ and progression of disease $(p<0.001)$. Age was inversely correlated to need for revision surgery $(p=0.035)$. Risk factors for the development of kyphosis were anterior graft failure $(p=0.040)$, vertebral body fracture $(p=0.012)$ and rod failure $(p=0.002)$. Rod failure was correlated with graft failure $(p<0.001)$ and vertebral body fracture $(p=0.002)$. Anterior reconstruction did not improve local control $(p=0.370)$ or reduce risk of kyphosis $(p=0.708)$ or need for revision $(p=0.434)$. For tumors in the thoracic spine the likelihood ratios of developing kyphosis was $7.4(p=0.025)$ and need for revision was $7.8(p=0.021)$ when compared to the cervical and lumbar regions.

Conclusion: Risk for revision include younger patients, development of kyphosis, graft failure, vertebral fracture, and rod failure. Since rod failure is correlated to risk of graft failure and fracture, surgeons should consider methods to reduce rod failure rates, especially in the higher risk thoracic region. Anterior reconstruction did not impact outcomes. 
9:16 - 9:18 am

\section{Rates of Inpatient Palliative Care Consultation for Patients with Brain and Spine Metastases}

Elizabeth P. Howell BS; Meghan Price; Tara Dalton; Theresa Williamson; Vikram Mehta MPH, MD; Brice Painter; CR Goodwin

Introduction: Brain and spine metastasis (BSM) patients are faced with complicated decision-making balancing intervention options, end-of-life planning, and high symptom burden. Palliative Care (PC) has been shown to provide support with symptom management, end-of life planning, and communication between patients, caregivers, and providers. Despite the benefits of PC, this resource remains infrequently utilized within the BSM patient population.

Methods: Analysis was performed of the rates of PC consults for BSM patients in a cohort of inpatients admitted to three associated tertiary medical centers. Patients were identified using ICD-9 and -10 codes corresponding to "brain metastases" and "bone metastases". All PC consults placed while a BSM patient was admitted inpatient were captured. PC referrals made after discharge were not captured by this analysis.

Results: 2608 total discharges were analyzed (2397 brain metastasis, 301 spine metastasis discharges). Average number of inpatient brain and spine metastasis patients receiving PC consult was $13.6 \%$ and $11.0 \%$ over this 3.5 year period, respectively. The large academic center analyzed had the lowest utilization - with $6.4 \%$ of brain and $7.6 \%$ of spine metastasis patients receiving PC consults - as compared to two smaller centers. Forboth brain and spine cohorts, Neurology was the discharging service with the highest rates of PC utilization, consulting PC for $37.7 \%$ of brain and $42.9 \%$ of spine metastasis patients respectively. Surgery, Neurosurgery, and Medicine had the lowest rates of PC consultation. Over time, PC utilization increased for brain, but not spine, metastasis patients.

Conclusion: PC consultation rates remain low amongst patients with brain and spine metastases, despite the high symptom burden and low overall survival for many of these patients. Ultimately, these single-institution utilization patterns may provide a microcosm for national patterns and trends at similar institutions. 
9:18 - 9:20 am

\section{Single-cell RNA Sequencing of Prostate Metastases to the Spine Reveals an Immunosuppressive Bone Marrow Microenvironment}

John H. Shin MD; Ninib Baryawno; Philip Saylor; David Sykes; David Scadden; Peter Kharchenko

Introduction: Cancer-related mortality due to non-hematologic malignancies is due to the development and progression of metastases. In advanced prostate cancer, metastases most often involve the spineleading to significant disability, pain, and loss of function.

Methods: Bone marrow and tumor samples obtained from spine surgeries were used to profile marrow and metastatic tumors from men with advanced prostate cancer at single-cell resolution. We contrasted the cellular composition and transcriptional states in matched samples of tumor and liquid bone marrow collected at the index and adjacent vertebral body levels, as well as bone marrow from iliac crests of patientsundergoing elective degenerative spine surgery (controls).

Results: Our analyses revealhematopoietic suppression and multifaceted immune distortion in the bone marrow. There was exhaustion of specific T-cell subsets, appearance of inflammatory lymphocytes and macrophages, and alteration of cytokine profiles. The chemokine CCL20 was notably overexpressed by myeloid cells, as was its cognate CCR6 receptor on T-cells. This dual overexpression was associated with repressed immune responses. We used a syngeneic mouse model of bone-metastatic prostate cancer to explore this observation, and demonstrated that disruption of the CCL20-CCR6 axis resulted in significant prolongation of survival. Overall, comparative high-resolution analysis of bone marrow reveals distinctive alterations associated with prostate cancer bone metastases that may be amenable to therapeutic targeting to alter cancer progression.

Conclusion: These datareveal a mechanism by which myeloid cells in the bone metastasis microenvironment can reduce effectiveness of cytotoxic T-cell populations through CCR6-coupled signaling. In addition to suggesting potential therapeutic avenues, our results demonstrate a pattern of widespread disruption of the human bone marrow by prostate-origin metastasis. This establishes a model for exploring other potential signaling and cellular relationships that inhibit immune response and remodel bone marrow into a tumorpermissive microenvironment. 
9:20 - 9:22 am

\section{MIS versus Open Surgery for Intradural-Extramedullary Spinal Cord tumor Resection: A Systematic Review and Meta-Analysis}

Soliman Oushy MD; Yagiz U. Yolcu MD; Amika Kamath; Waseem Wahood; Anshit Goyal MBBS; Mohammed A. Alvi MD; Mohamad Bydon MD

Introduction: Intradural extramedullary spinal cord tumors account for $40 \%$ of all spinal cord tumors as the second most common tumors following extradural tumors. Mostly, the initial choice of treatment for ID-EM spinal cord tumors is a gross total resection. Given the susceptibility of spinal cord to injury during surgery, minimally invasive surgical techniques have emerged as alternatives to conventional open surgery in resection of ID-EM tumors.

Methods: An electronic database search was conducted and the review was carried out according to PRISMA guidelines and recommendations. Inclusion criteria were as follows; (i) comparative studies of MIS vs OS; (ii) studies reporting outcomes for patients undergoing surgery for ID-EM tumors. Variables collected were patient demographics, estimated blood loss (EBL), Mean operative time (Minutes), length of stay (days),

complications, extent of tumor resection. These outcomes were adjusted for tumor type and size with a metaregression analysis.

Results: The search identified a total of 275 studies. After the selection criterion was applied 8 comparative studies were included in this review. A total of 424 patients were included in the analysis with $202(47 \%)$ of them undergoing MIS and 222 of them (53\%) undergoing open surgery. EBL and complication rates were significantly lower in Mls group ( $p=0.0002$ and $p<0.0001$, respectively). Two groups were similar with regards to operative time, length of hospital stay and extent of resection (categorized as gross total or sub-total resection).

Conclusion: Results of this meta-analysis show a significant reduction in blood loss and complications with minimally invasive spine surgery while preserving high rates of gross-total resection. The findings suggest that the minimally invasive spine surgery may serve as a beneficial alternative for patients undergoing spine surgery for ID-EM tumors of the spinal cord. 
9:22 - 9:24 am

\section{The Effect of Pre-operative Opioid Use On Hospital Length of Stay in Patients Undergoing Elective Spine Surgery}

Eve C. Tsai MD, PhD, FRCS(C), CIP; Alexandra Stratton; Sarah Tierney; Eugene Wai

Introduction: The opioid epidemic has placed a significant social and economic burden on our healthcare system, resulting in opioid-dependent, complex pain patients arriving more routinely for surgery. The relationship between opioid use prior to spine surgery and hospital length of stay (LOS) remains an unclear, yet important issue.

Methods: A retrospective chart review was conducted on consecutive adult patients admitted following elective spine surgery and seen by the acute pain service at a single institution for one year. Post-operative LOS, demographic variables and pre-operative analgesics used (including dose) were obtained. The maximum prescribed opioid dose was converted to oral morphine equivalents (MEQ) and patients whose daily dose was $\geq 90 \mathrm{MEQ}$ were considered high opioid users. Multivariate logistic regression analyses were performed to assess the effect of opioid use and dose on LOS, while adjusting for confounders.

Results: Analysis included 220 patients, 111 (50.5\%) of whom were not prescribed opioids pre-operatively (non-opioid group) while 109 (49.5\%) were prescribed opioids pre-operatively (opioid group). The median maximum prescribed daily dose was $30 \mathrm{MEQ}$ (range 1.2-480 MEQ). A moderate correlation was found between LOS and opioid dose $(R=0.21, p=0.0019)$. Patients were categorized based on amount of preoperative opioids: non-opioid, low-mid and high dose. High opioid users had significantly longer LOS compared to the other two groups $(p<0.05)$.

Conclusion: High-dose pre-operative opioid use was associated with a longer LOS in patients undergoing elective spine surgery requiring admission at our single tertiary care centre. These findings provide support for implementation of interventions to decrease opioid use and LOS in spine surgery patients. Future research should assess the costs and benefits of such interventions. 


\section{9:24 - 9:26 am}

\section{Preoperative Radiation is an Independent Predictor of Time to Death in Metastatic Spine Patients}

Patricia E. Zadnik; Ahmed Albayar; Ashwin G. Ramayya MD, PhD; Stephen Miranda MD; Jang Yoon MD, MS; James M. Schuster MD, PhD; Zarina S. Ali MD; Neil R. Malhotra MD; Ali K. Ozturk MD

Introduction: Patients with metastatic spinal disease require coordinated care. As radiation protocols are applied to more complex tumors, the precise timing for surgical intervention must be explored.

Methods: We examined a retrospective cohort of 109 patients undergoing surgery for spinal metastatic disease. Seventy-five patients did not undergo radiation to the surgical site before surgery (NPR), while thirtyfour patients received preoperative local radiation (PR). We performed a survival analysis using a multivariate Cox Proportional Hazards to assess significant predictors of time to death even when accounting for other clinical factors. We then looked at surrogate markers of preoperative health, including age, preoperative ASA, KPS and BMI. Chi-squared testing was used for comparison of means and proportions.

Results: Local radiation before surgery $(\mathrm{HR}=2.1, p=0.02)$ and primary lung cancer $(\mathrm{HR}=2.3052, p=0.04)$ were significant predictors of time to death on multivariate analysis when controlling for other factors. There was no significant difference in the rate of lung cancer between NPR and PR groups $(p=0.89)$; ten patients in the no preoperative radiation (NPR) group (13\%) had lung cancer while four patients in the preoperative radiation (PR) group had lung cancer $(12 \%)$. PR versus NPR cohorts did not exhibit statistically significant differences in age $(p=0.22)$, preoperative BMI $(p=0.28)$, ASA $(p=0.12)$. NPR patients had more postoperative wound complications (12\% versus $0 \%$ in PR group).

Conclusion: While this study is limited by its small sample size, preoperative radiation remained a robust, independent predictor of time to death. On closer examination, preoperative KPS, as well as surrogate factors of preoperative health such as BMI and ASA score did not explain these differences. 
9:26 - 9:28 am

\section{Characterizing the Rates and Causes of Spinal Cord Compression in Patients with Known Spinal Metastases}

Joshua Benton; Rafael De la Garza Ramos MD; Yaroslav J. Gelfand MD; Phillip C. Cezayirli MD; Murray Echt MD; Reza Yassari MD, MS; Vijay Yanamadala MD, MBA

Introduction: Spinal cord compression secondary to metastatic disease can cause irreversible spinal cord damage and neurological deficits. Preventing metastatic spinal cord compression (MSCC) in patients with known spinal metastases (SM) can drastically improve their quality of life and long-term outcomes.

Methods: Adult patients treated at our institution between 2017-2019 with SM were identified from our institutional database. Patients charts were reviewed to determine if they presented to the hospital with acute focal neurological deficits secondary to radiographically diagnosed MSCC. In patients with MSCC, the nature (i.e. motor versus sensory) and degree of acute neurological deficit were recorded. The time between the first radiographic image demonstrating SM and the onset of MSCC was recorded. Additionally, their charts were reviewed to determine possible barriers to care between the initial identification of SM and the diagnosis of MSCC.

Results: A total of 118 patients were identified from our database. Twenty four patients (20.3\%) presented to the hospital with new focal motor and/or sensory deficits secondary to MSCC.Eight patients $(6.8 \%)$ had prior imaging demonstrating SM. These patients experienced a median of 66 days (IQR $58.3-172.8$ ) between the initial identification of SM and development of MSCC. The most common barriers to MSCC prevention they experienced were failed treatment (50\%), loss to follow-up (37.5\%), misdiagnosis $(12.5 \%)$, and social barriers $(12.5 \%)$.

Conclusion: Patients with proven SM may encounter barriers to care that increase their risk of developing MSCC. Further evaluation of and changes to current care pathways for patients with SM are needed to optimize their care. 
9:28 - 9:30 am

\title{
Minimally Invasive Surgery for Intradural Extramedullary Spinal Cord Pathologies: A Case Series
}

\author{
Ryan Kelly; Islam Fayed MD, MS; Anthony G. Conte MD; Amjad N. Anaizi MD
}

Introduction: Intradural extramedullary spinal pathologies have traditionally been treated with open approaches. However, minimally invasive surgery (MIS) has become an increasingly common method for treating a variety of spinal pathologies. Intradural lesions present a particular challenge due to the need for an intended durotomy that can increase the risk of CSF leak.

Methods: We conducted a single-center retrospective review of patients who underwent resection of intradural spinal cord lesions via a MIS approach using fixed 20-millimeter tubular retractors. Dural closure was achieved through the use of standard operating room equipment. Demographic, perioperative, and outcomes data was collected and analyzed.

Results: Nine patients with intradural spinal cord pathologies were included in the present study. Five (55.6\%) patients were female, with a mean age of $47.78( \pm 19.62)$ years and a mean BMI of $28.75( \pm 9.24)$. The pathologies consisted of 3 schwannomas, 4 arachnoid cyst/webs, 1 ependymoma, and 1 bullet fragment. Mean procedure time was $190.67( \pm 51.50)$ minutes with a mean estimated blood loss of $40.00( \pm 19.27) \mathrm{mL}$. Mean length of stay was $2.89( \pm 2.98)$ days with a mean follow-up time of $9.57( \pm 10.77)$ months. No perioperative complications were noted, and no patients were readmitted for any reason following surgery.

Conclusion: Intradural extramedullary pathologies can be safely and effectively resected with subsequent water-tight closure of the durotomy using MIS with fixed tubular retractors and standard operating room equipment. 


\section{Sunday, March 8, 2020 \\ General Spine Surgery 3/Peripheral Nerve/Basic Science Abstract Breakout Session}

\section{7:30 - 7:32 am}

\section{The Association of Education on Patient-Reported Outcomes after Elective Lumbar Spine Surgery: A MSSIC Study}

Mohamed Macki MD, MPH; Travis M. Hamilton MD; Michael Bazydlo MS; Lonni Schultz PhD; Hesham M. Zakaria MD; Jad Khalii; Miguelangelo Perez-Cruet; Ilyas Aleem MD, Msc, FRCSC; Paul Park MD; David Nerenz PhD; Jason M. Schwalb MD, FAANS, FACS; Muwaffak Abdulhak MD, FRCS; Victor W. Chang MD

Introduction: Underlying socioeconomic factors invariably alter patient-reported outcomes (PRO) after spine surgery. One such modifier, education level, has been poorly studied.

Methods: Michigan Spine Surgery Improvement Collaborative (MSSIC) was queried for all lumbar operations. PROs were determined by NASS Patient Satisfaction Index and Minimally Clinically Important Difference in Oswestry Disability Index (MCID in ODI). Multivariable Poisson generalized estimating equation (GEE) models reported adjusted risk ratios $\left(R R_{a d j}\right)$.

Results: Of the 21786 patients, post-college cohorts $(\mathrm{N}=2458)$ experienced highest rates of satisfaction versus <HS/GED ( $\mathrm{N}=1351)$, HS/GED ( $\mathrm{N}=10665)$, and college $(\mathrm{N}=7312)$ education at 90-days, 1-year, and 2years postoperatively. Likelihood of postoperative 90 -day satisfaction significantly increased by $10 \%$ $\left(R_{\mathrm{adj}}=1.10\right.$ [1.05-1.15]) among HS/GED, 12\% $\left(\mathrm{RR}_{\mathrm{adj}}=1.12\right.$ [1.06-1.17]) among college, and $13 \%\left(\mathrm{RR}_{\mathrm{adj}}=1.13\right.$ [1.08-1.19]) among post-college education compared to <HS/GED. At 1-year, satisfaction significantly increased by $11 \%\left(R_{a d j}=1.11\right.$ [1.05-1.21]) among college and 17\% $\left(R R_{a d j}=1.17\right.$ [1.07-1.27]) among postcollege education compared to <HS/GED; no association with HS/GED. Statistically significant predictors of 2year satisfaction could not be elicited.

Likelihood of postoperative 90-day MCID in ODI significantly increased by $14 \%\left(\mathrm{RR}_{\mathrm{adj}}=1.14\right.$ [1.05-1.25]) among college and 18\% (RR $\mathrm{Rdj}_{\mathrm{a}}=1.18$ [1.09-1.29]) among post-college education compared to <HS/GED; no association with HS/GED. At 1-year, MCID in ODI significantly increased by $15 \%\left(R R_{a d j}=1.15\right.$ [1.02-1.29]) among HS/GED, 24\% (RR $\mathrm{Rdj}_{\mathrm{j}}=1.24$ [1.11-1.38]) among college, and 32\% ( $\mathrm{R}_{\mathrm{adj}}=1.32$ [1.17-1.49]) among postcollege education compared to <HS/GED. At 2-years, MCID in ODI significantly increased by $25 \%\left(R R_{a d j}=1.25\right.$ [1.02-1.54]) among HS/GED, 33\% ( $R_{\mathrm{adj}}=1.33$ [1.10-1.62]) among college, and 48\% ( $\mathrm{R}_{\mathrm{adj}}=1.48$ [1.21-1.82]) among post-college education compared to <HS/GED.

Conclusion: Reaching MCID for ODI, and satisfaction with surgery appeared to correlate with level of education where post-college educate patients had the best outcomes, and those with less than high school education fared the worst. This suggests a potential disparity linked to education in elective spine surgery. 
7:32 - 7:34 am

\section{Impact of Body-Mass Index on Clinical and Radiographic Outcomes Following Posterior Cervical Fusion Surgery}

Nida Fatima; John H. Shin MD

Introduction: With the increasing worldwide prevalence of obesity, the rate of degenerative spinal disease among patients with high body mass index (BMI) is also accentuating.

Methods: A retrospective chart review of 199 patients presented at an academic tertiary care hospital between 2009-2019 was carried out. Patients were categorized into 4 groups based upon the BMI as (i) normal weight

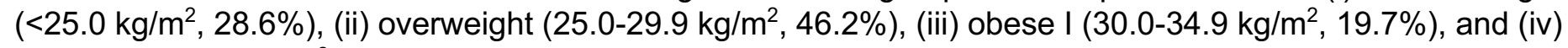
obese II-III ( $\left.\geq 35 \mathrm{~kg} / \mathrm{m}^{2}, 5.6 \%\right)$. For each BMI cohort, univariable association with postoperative outcomes were first determined using logistic regression, and a prediction model was then constructed using all characteristics significant at the 0.30 alpha level. All associations were reported as odds ratio (OR) with $95 \%$ confidence interval $(\mathrm{Cl})$.

Results: Overall no significant association existed between the BMI spectrum, and postoperative neurological outcomes and complications $(p<0.05)$. However, multivariable regression analysis revealed that obese I patients had a 2.4-folds higher likelihood of postoperative C2-C7 SVA >4 cm (OR: 2.44, 95\%Cl: 1.21-4.94, $\mathrm{p}=0.01$ ). Furthermore, patients categorized into Obese II-III had $92 \%$ less likelihood of T1 slope $>25^{\circ}$ postoperatively (OR: $0.08,95 \% \mathrm{Cl}: 0.01-0.43, p=0.003$ ).

Conclusion: Posterior fusion surgery following DCSD is both safe and effective for patients across the entire spectrum of BMI. Our results corroborate that, regardless of the BMI, all patients should be counseled to expect the comparable rate of complications and neurological improvement. 


\title{
7:34 - 7:36 am
}

\section{Utility of Venous Thromboembolism Chemoprophylaxis: An Institutional Analysis}

\author{
Allen L. Ho MD; Austin Y. Feng BS; Paras Fatemi; Zachary A. Medress MD; Atman Desai MD, MA; Anand \\ Veeravagu MD; John K. Ratliff MD, FACS
}

Introduction: Venous thromboembolism (VTE) is a serious complication in spine surgery. However, there is a lack of consensus regarding the efficacy VTE chemoprophylaxis, and current guidelines recognize the need for further studies to evaluate the use of chemoprophylaxis as well as the optimal agent and dosing strategies in elective spine patients.

Methods: A retrospective chart review of elective posterior lumbar fusion patients at a major academic medical center from 2017-2018. Tumor and trauma patients, as well as patients receiving combined (anterior) approaches or osteotomies were excluded. Primary outcomes were development of deep vein thrombosis (DVT), pulmonary embolism (PE), and post-operative hematomas as well as presence and type of VTE prophylaxis (e.g. aspirin (ASA), subcutaneous heparin (SQH), etc.). Secondary measures include length of stay (LOS), first ambulation post-operative hours, length of chemoprophylaxis use, drain usage, and blood transfusion status. For significance testing, Students T-test and Fishers exact test are used.

Results: A total of 473 patients were included, with 248 receiving VTE chemoprophylaxis (73.8\% SQH, 21.8 ASA, and $4.4 \%$ Lovenox). All patients received sequential compression devices (SCDs) and early postoperative mobilization/ambulation whenever possible. Rates of DVT, PE and hematoma are between the chemoprophylaxis $(0.8 \%, 0.4 \%$, and $0.4 \%)$ and non-chemoprophylaxis groups $(1.3 \%, 0.9 \%$, and $1.3 \%)$ and were not significantly different $(P=0.6718,0.606$, and 0.3498 , respectively). Patients with VTE prophylaxis have significantly longer length of stay and first ambulation post-operative hours $(P<0.001$ and $P<0.05)$, but not length of drain usage, and units of transfused blood $(P>0.05)$.

Conclusion: Chemoprophylaxis did not significantly reduce VTE for elective posterior lumbar fusions at our institution. This suggest that SCDs and early mobilization may be sufficient for VTE prophylaxis, though further investigations in wider patient cohorts are warranted. 
7:36 - 7:38 am

Posterior Cervical Fusion for Treatment of Atlantoaxial Arthrosis: Case Series and Review of Literature

Andrew Platt MD; Brian T. David PhD; Victoria D. Vuong; John E. O'Toole MD, MS; Vincent C. Traynelis MD

Introduction: Atlantoaxial arthrosis is characterized by unilateral neck pain that radiates to the occiput and is exacerbated with axial rotation. Patients who are not responsive to non-operative treatment may benefit from cervical fusion.

Methods: All patients who underwent atlantoaxial posterior fusion for atlantoaxial arthrosis between 2010 and 2018 were included. Patients were excluded if they underwent C1-2 fusion for any other indication. Primary outcome measures included Neck Disability Index (NDI), Visual Analogue Scale-Neck Pain (VAS-N), and frequency of postoperative complications.

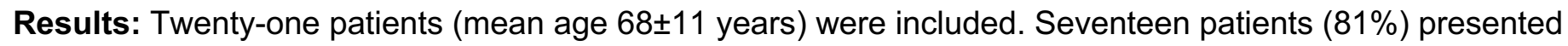
with unilateral neck pain exacerbated with axial rotation. Eleven patients (52\%) presented with radiographic signs of C1-2 instability. CT was the most helpful imaging study. Four patients had preoperative nuclear medicine bone scans with a discrete unilateral focus of uptake lateralizing to the concerning C1-2 joint. Average follow-up was $15.29 \pm 9.8$ months. Preoperative Neck Disability Index scores decreased from 42.5 to 38.7 $(p=0.58)$ at final follow-up. Visual Analog Scale-Neck (VAS-N) scores significantly decreased from 7.4 preoperatively to $4.6(p=0.004)$ at final follow-up. Postoperative complications included dysphagia $(9.5 \%)$, surgical site infection (4.8\%) and one case of mortality within the same admission from endocarditis. There were two cases $(9.5 \%)$ of reoperation, one for translaminar screw removal, and one for evacuation of hematoma.

Conclusion: Atlantoaxial fusion is an effective treatment for atlantoaxial arthrosis. Preoperative nuclear medicine bone scans may help identify patients with higher rates of postoperative improvement. 
7:38 - 7:40 am

\section{Medicare Utilization Trends for Spinal Cord Stimulators: 2010-2018}

Jack M. Haglin BA; Kent Richter; Jordan Pollock; Jakub Godzik MD, MSc; Naresh P. Patel MD

Introduction: Spinal cord stimulation is becoming an increasingly popular modality for treating chronic pain.

Methods: The 2010 and 2018 Physician Supplier files from the Centers for Medicare and Medicaid website were queried regarding each of the three current procedural terminology (CPT) codes for spinal cord stimulator implantation. Data was stratified by provider type, and data for the top three provider types were collected for each code. The number of services billed to Medicare for each CPT code both in total, and by provider type was collected for years 2010 and 2018, and the differences were analyzed.

Results: Total Medicare utilization of spinal cord implantation procedures has increased $149.1 \%$ overall from 2010 to $2018(90,281$ in 2010 to 224,865 in 2018). Utilization for CPT code 63650(Percutaneous implantation of neurostimulator electrode array) has increased from 61,340 in 2010 to 151,687 procedures in 2018(+ 90,347 procedures, $+147.3 \%$ ). Utilization for CPT code 63655 (Laminectomy for implantation of neurostimulator electrodes) has increased from 9,152 procedures in 2010 to 18,563 procedures in 2018(+ 9,411 procedures, $+102.8 \%$ ). Utilization for CPT code 63685 (Insertion of spinal neurostimulator pulse generator)has increased from 19,789 procedures in 2010 to 54,615 procedures in $2018(+34,826$ procedures, $+176.0 \%)$. Across all procedures, Neurosurgeons accounted for $12.1 \%$ of all billing providers in 2010 , and $10.3 \%$ of all billing providers in $2018(-1.8 \%)$.

Conclusion: This study demonstrates that Medicare utilization for spine stimulator implantation procedures is increasing since 2010. Further, when evaluating by provider type, neurosurgeons are accounting for less of the total billing market compared to other specialties. This data is important for neurosurgeons and policy leaders to define the growing demand for spinal cord stimulators, and to assure adequate resources are available to meet this demand. 


\title{
7:40 - 7:42 am
}

\section{Medicare Reimbursement of Implantable Spinal Cord Stimulators from 2000-2019}

\author{
Kent Richter; Jack M. Haglin BA; Jordan Pollock; Jakub Godzik MD, MSc; Naresh P. Patel MD
}

Introduction: Chronic back pain is one of the most common ailments of individuals within the United States. There are a variety of procedures and devices designed to improve back pain depending on the etiology of the pathologic process. One such device is the implantable spinal cord stimulator. Currently, there is no literature evaluating procedural reimbursement for implantation of spinal cord stimulation.

Methods: CPT reimbursement codes $63650,63655,63680$ for spinal cord stimulation were identified using Medtronic Reimbursement Services. Subsequently, reimbursement of these CPT codes was identified using the Physician Fee Schedule Look-Up Tool from the Centers for Medicare Medicaid Services. The raw percent change in Medicare reimbursement rate from 2000 and 2019 was calculated for each procedure and averaged. This was then compared to the percent change in consumer price index over the same time. Using data adjusted for inflation, trend analysis was performed for all included procedures.

Results: After adjusting all reimbursement data for inflation, the average reimbursement for included procedures decreased by an average of $-36.71 \%$ between 2000 and 2019 . Code 63650 (percutaneous implantation of neurostimulator electrode array) decreased by $-35.99 \%$. Code 63655 (Laminectomy for implantation of neurostimulator electrodes, plate/paddle) decreased by $-24.87 \%$. Code 63685 (Insertion or replacement of spinal neurostimulator pulse generator or receiver, direct or inductive coupling) decreased by $49.27 \%$.

Conclusion: This is the first study to evaluate trends in procedural Medicare reimbursement for Implantable Spine Stimulation devices. When adjusted for inflation, Medicare reimbursement for included procedures has decreased significantly from 2000 to 2019. Increased awareness and consideration of these trends will be important as hospitals and physicians continue to provide palliative care for patients who suffer from chronic back pain. 
7:42 - 7:44 am

\section{Robot Assisted Neuro-navigation for Pedicle Screw Fixation: Protocol Modification for the Procedure Optimization}

Komal Naeem MD; Malika Bhargava ME, MBA, MD; Randall W. Porter MD

Introduction: Robot assisted neuronavigation improves the precision and accuracy of pedicle screws via realtime feedback provided by the visualization of the instrument. It can also potentially decrease the radiation exposure and operative duration. However, like any other novel technology, it also has a learning curve.

Methods: All the patients undergoing robot assisted neuronavigation for pedicle screw placement at our hospital performed by single surgeon were included in the study. Data were collected prospectively, recording time required for each step, intraoperative details, challenges encountered with their solutions. Gertzbein Robbins classification was used for assessing pedicle screw accuracy. Grades A and B were considered clinically acceptable.

Results: We enrolled 26 patients prospectively. Data for operative duration was available for 20 patients and a $34 \%$ average decrease in time was observed with performing Computed tomography (CT) pre-operatively instead of intra-operatively $(p=0.02)$. It also proved to decrease radiation dose significantly $(p=0.04)$. We did not report any incident of skiving after replacing the drill bit with a burr (4 vs. 0 ). Additionally rate of screw repositioning was improved from $6.5 \%$ to $2.2 \%$.

Conclusion: Performing pre-operative CTs, planning screw pre-operatively and replacing drill bit to burr have proven to make the technique more efficient. This shows the importance of learning curve and continuous strive for the improvement with a novel technology. 
7:44 - 7:46 am

\title{
National Utilization Trends in Single and Co-Surgeon Surgeries for Single Level Posterior Lumbar Interbody Fusions
}

\author{
Shashank Vijay Gandhi; Daniel Toscano; Ahmad Latefi DO
}

Introduction: With the growing cost of healthcare, it is important to understand trends in surgical practices. The presence of co-surgeons has been present for years in spinal surgeries; however, the trends for posterior lumbar interbody fusions has yet to be characterized.

Methods: Medicare Part B Physician/Supplier Procedure Summaries from 2012-2018 were analyzed for all CPT code 22633 (posterior lumbar interbody fusion) and 22633-62 (co-surgeon modifier). The total procedure volumes, costs, and utilization rates were assessed for the Medicare fee-for-service population.

Results: The single surgeon and co-surgeon utilization for posterior lumbar interbody fusions per 100,000 Medicare beneficiaries has increased $33 \%$ and $35 \%$ since 2012, respectively. Even with the increase in CPT codes submitted, the use of the co-surgeon modifier has remained consistent around $3.3 \%$ of all single-level posterior lumbar interbody fusion cases. The charges submitted per 100,000 beneficiaries has increased $47 \%$ for single surgeons and $43 \%$ for co-surgeon cases. A total of $\$ 10,680,707$ was charged for co-surgeon cases in 2018 , amounting to a utilization of $\$ 27,598$ per 100,000 Medicare beneficiaries. If these cases had been done by a single surgeon, the total savings would have amounted to $\$ 2,136,141$, with a potential utilization savings of $\$ 5,519$ per 100,000 beneficiaries. There was no difference in trends between neurosurgeons and orthopedic surgeons.

Conclusion: The volume of single surgeon and co-surgeon utilization for single-level posterior lumbar interbody fusions has increased from 2012 to 2018 . The co-surgeon modifier as consistently been used on average $3.3 \%$ of all cases, with a relatively small increase in costs per 100,000 beneficiaries. This small increase, which may be justified in the Medicare population, may not be as easily done with commercial insurers, who have higher charges. 
7:46 - 7:48 am

\section{The Use of the Hospital Frailty Risk Score to Predict Outcomes Following Anterior and Posterior Cervical Fusions}

Rebecca Baron; Sean N. Neifert BS; Michael Martini; Georgios A. Maragkos MD; Ian T. McNeill MD; Eric Oermann; John Caridi

Introduction: The Hospital Frailty Risk Score (HFRS), developed using International Classification of Diseases (ICD) codes, is an effective tool for identifying patients at risk of poor outcomes.

Methods: The National Inpatient Sample (NIS) was queried for ACDF and PCDF hospitalizations between 2013-2016 based on ICD-10 and converted ICD-9 codes. The HFRS was used to stratify patients into low, medium, and high frailty groups. Patients were compared on the basis of demographics and outcomes.

Results: The results found that patients with higher HFRS correlated with higher comorbidity burden (Elixhauser Index score $\geq 5$ ) in both procedural cohorts $(p<0.0001)$. Mean mortality rates were greatest in the high HFRS versus medium and low HFRS groups for both ACDF (8.0\% vs 1.9 and $0.04 \%$, respectively) and PCDF procedures ( $p$ 's<0.0001). The mean length of stay (LOS) was longer in the high versus medium and low HFRS groups following ACDF (20.6 vs 7.7 and 1.7 days) and PCDF procedures ( $p$ 's<0.0001). The high HFRS group had greater mean total in-hospital charges for anterior fusions when compared to medium and low HFRS groups $(\$ 280,092$ vs $\$ 133,492$ and $\$ 63,425)$, with a similar trend for posterior fusions $(p ' s<0.0001)$.

Conclusion: These results reveal that high HFRS correlates with increased mortality risk, longer length of stay, and greater healthcare expenditure in patients undergoing ACDF and PCDF. Further research into frailty stratification techniques could guide effective resource utilization and improve outcomes. 
7:48 - 7:50 am

\section{Effect of Simulation Training on Resident Proficiency in Placement of Navigated Thoracolumbar Pedicle Screws}

Andrew M. Gardeck BA; Xuan Pu BS; Qiuyu Yang BS; David W. Polly MD; Kristen E. Jones MD;

Introduction: No standard exists for the safest, most efficient method of training residents to place spinal instrumentation.Synthetic spine models havesimilarutility ratings, low variability, and high availability compared to cadaveric labs and virtual simulators. Computer-assisted navigation further improves screw accuracy but is rated as difficult to use by learners.

Methods: Fifteen neurosurgery/orthopedic surgery residents participated in a standardized curriculum with lectureandtwo separate sessions of thoracolumbar pedicle screw placement in a syntheticmodel utilizing threedimensional computer-assisted navigation. Data was collected on pre-module experience, time and accuracy of screw placement. Participants were scored objectively using OSATS and subjectively using PPDIS. Pre and post-module scores were compared.

Results: 15 of $15(100 \%)$ residents demonstrated statistically significant improvement in subjective/PPDIS and objective/OSATS proficiency in screw placement with this curriculum $(p<0.001)$, regardless of prior experience. Fourteen of fifteen residents demonstrated decreased time per screw placement from session 1 to session 2 outside of expected improvement based on repetition alone, without significant difference in accuracy between session 1 and session 2 .

Conclusion: A standardized curriculum using synthetic models results in significantly improved resident subjective and objective proficiencyfor navigated thoracolumbar pedicle screw placement. This can assist in development of a nationwide competency curriculum for safe, efficient resident training in spinal instrumentation placement. 
Spine Summit Meeting Abstracts, May 2021

7:50 - 7:52 am

\section{Comparative Analysis of Transforaminal Endoscopic Discectomy and Microscopic Discectomy for Thoracic Disc Herniation}

Jun Seok Bae; Sang-Ho Lee MD, PhD

Introduction: Symptomatic thoracic disc herniation (TDH) is rare. None of the reported surgical technique has been accepted universally.

Methods: 77 patients (55.9 years, 11.2 months FU) withTDHwere retrospectively reviewed. 39 patients (49.5 years, 28 males) were treated by TETD under local anesthesia and 38 patients (62.5 years, 21 males) were treated by MD under general anesthesia. Radiological factors were reviewed. The VAS score for dorsal back pain (DBP) and ODI was used for the evaluation of clinical and functional outcomes. The patients satisfaction was evaluated with Modified Macnabs criteria.

Results: The levels of surgery were evenly distributed in the TETD group (15\% upper thorax, 51.2\% mid thorax and 33.3\% lower thorax) than in the MD group (7.8\% upper, $26.3 \% \mathrm{mid}, 65.7 \%$ lower thorax). The location of hernia was similar in posterolateral $(66.5 \%, 68.4 \%)$ and central disc $(17.9 \%, 18.4 \%)$ in TETD and MD, respectively. Partial calcification of hernia was observed in $30.7 \%$ and $28.9 \%$ in TETD and MD, respectively. Operation time (75min, $170 \mathrm{~min})$, EBL $(0 \mathrm{ml}, 357 \mathrm{ml})$ and hospital stay (4.4days, 13days) showed a significant difference $(p<0.05)$. VAS score for dorsal back pain $(7.5$ vs $2.5,7.9$ vs 2.7$)$ and ODI score (47.6 vs $13.7,43.2$ vs 14.7$)$ were significantly improved in both groups. TETD patients tended to be more satisfied with the outcome in terms of Macnab's criteria $(89 \%$ vs $71 \%, p=0.070)$. Two patients in the MD group underwent revision surgery due to incomplete decompression and a postoperative hematoma on the next day of surgery. One patient in TETD underwent MD due to incomplete decompression.

Conclusion: TETD for theTDHis a feasible and safe procedure that could be addressed for a wider range of surgical levels with lesser operation time and hospital stay and insensible bleeding. While achieving similar outcomes, TETD achieved better satisfaction of patients due to local anesthesia and its minimally invasive approach. 
7:52 - 7:54 am

\section{Does Attachment of the Dorsolumbar Fascia to a Spinous Process Prosthesis Affect the Kinematics of the Lumbosacral Spine}

Daina Brooks; Bryan W. Cunningham; Mohit Kukreja; Ken Mullinix; Nicholas Rolle; P. Justin Tortolani

Introduction: Post-laminectomy kyphosis, disc herniations, and/or spondylolisthesis are documented complications following multi-level laminectomy. Removal of the spinous processes and ligaments in multi-level laminectomy disrupt the normal kinematics of the spine, increase segmental motion and risk of hypermobilityrelated complications.

Methods: Eight lumbopelvic spines (T9-sacrum) were utilized in this investigation. Specimens retained all paraspinal musculature, ligaments, fascia and skin layers. Multidirectional flexibility testing was performed at $\pm 10 \mathrm{Nm}$ in flexion-extension, lateral bending, and axial rotation. Reconstructions included L2-L4 laminectomy, L2-L4 laminectomy with fascia/skin closure, and L2-L4 SP-P $(n=3)$ attached to pedicle screws with fascia closure to prosthesis. ROM at the superior adjacent (T12-L2), operative (L2-L5), and inferior adjacent (L5-S1) levels was quantified and compared.

Results: There were no statistically significant changes in ROM at the superior and inferior adjacent levels for all reconstructions. At the operative levels, flexion-extension motion was significantly greater for the laminectomy $(156.57 \pm 38.89 \%)$ and closed laminectomy $(147.11 \pm 35.93 \%)$ reconstructions when compared to intact. The laminectomy reconstruction also demonstrated significantly greater lateral bending motion $(110.65 \pm 6.43 \%)$ compared to intact. In axial rotation, the laminectomy (157.95 $\pm 18.27 \%)$, closed laminectomy $(160.54 \pm 21.39 \%)$, and SP-P $(159.36 \pm 18.38 \%)$ reconstructions observed greater motion when compared to intact $(p<0.05)$.

Conclusion: Re-establishing the posterior arch using spinous process protheses restored flexion-extension motion at the operative levels following multilevel laminectomy. 


\section{7:54 - 7:56 am}

\section{Accuracy of Cortical Bone Trajectory Screw Placement with Intraoperative Cone Beam Navigation}

Joseph Laratta; Jamal Shillingford; Andrew Pugely; Avery L. Buchholz MD; Mladen Djurasovic; Jeffrey L. Gum MD; Steven D. Glassman; Charles H. Crawford III MD

Introduction: Cortical bone trajectory (CBT) screws are typically inserted under fluoroscopic guidance with a medial-to-lateral trajectory axially and a caudal-to-cephalad trajectory sagittally. Toreduce surgeon radiation exposure and improve accuracy, CBT screws may be inserted under navigation with intraoperative cone bone CT. However, the accuracy of CBT screw placement under intraoperative navigation has yet to be assessed in the literature.

Methods: Consecutive patientswho underwent CBT fixation with 618 screws under intraoperative navigation were analyzed from May 2016 through May 2018. Screws were placed by one of three senior spine surgeons using Medtronic O-Arm Stealth Navigation. Screw position was assessed on intraoperative and postoperative CT scans using 2D and 3D reconstructions with VitreaCore software.

Results: There were 134 patients were included and most surgeries were primary (73.1\%). Ten violations of the vertebral cortex were noted with an average breach distance of $1.01 \pm 0.7 \mathrm{~mm}$. Three breaches were lateral $(0.6 \%)$ and seven were medial $(1.6 \%)$. The overall navigated screw accuracy rate was $98.3 \%$. The accuracy to within $1 \mathrm{~mm}$ of error was $99.2 \%$. There were no intra-operative neurologic, vascular, or visceral complications related to the placement of the screws.

Conclusion: CBT screw fixation under an intraoperative cone beam CT navigated insertion technique is safe and reliable. Despite five breaches greater than $1 \mathrm{~mm}$, there were no complications related to the placement of the CBT screws. 
7:58 - 8:00 am

\section{Promising Biomimetic Delivery System with Osteoinductive Bone Particles in Biodegradable Grafts For Spinal Fusion}

Alexander Perdomo-Pantoja MD; Alexandra Rindone; Christina Holmes; Kathleen Noller; Warren Grayson; Timothy F. Witham BS, MD

Introduction: Biodegradable biomaterials degrade as new bone tissue grows into the graft while also providing mechanical stability during the early stages of bone healing. Using a newly designed, biodegradable polycaprolactone-decellularized bone (PCL-DCB) hybrid, Rindone et al. developed a biomimetic plateletderived growth factor-BB (PDGF) delivery system using heparin-conjugated, decellularized bone particles (HCDCB) that enhances adipose-derived stem cells (ADSC)-mediated bone regeneration.

Methods: ADSCs were isolated from syngeneic rats and cultured in vitro. Cultured-expanded ADSCs (P2) were seeded onto PCL-DCB scaffolds along with HC-DCB particles (40 mg/mL in fibrin) and $20 \mathrm{ug} / \mathrm{mL}$ PDGF for subsequent transplantation. Dorsolateral spinal fusion surgery at L4-L5 was performed on Lewis rats divided into 5 experimental groups (2 control groups, and 3 treated groups): [1] PCL only (Control 1); [2] PCLDCB + HC-DCB particles (Control 2); [3] PCL-DCB + HC-DCB particles + PDGF; [4] PCL-DCB + 2.5x106 ADSCP2/side + HC-DCB particles; and, [5] PCL-DCB + 2.5x106 ADSCP2/side + HC-DCB particles + PDGF. Fusion was evaluated eight weeks post-surgery.

Results: MicroCT analysis revealed that fusion was reached in all treated groups $[3,4,5]$, achieving maximum CT fusion and manual palpation scores. No statistical differences in new bone volume were found between treated groups $(p>0.05)$, which was higher than in control groups $[1,2](p<0.05)$.

Furthermore, histological staining demonstrated that the groups with PCL-DCB grafts were well-integrated with the host transverse processes, showing expression of CD31, Osteocalcin, and RUNX2 in these groups.

Conclusion: Biodegradable PCL-DCB grafts enable spinal fusion when combined with HC-DCB particles and either ADSCs or PDGF-BB, suggesting that the presence of both ASCs and PDGF-BB is not required to achieve spinal fusion in PCL-DCB grafts if they are combined with osteoinductive HC-DCB. 
8:00 - 8:02 am

\section{Patient Rated Outcomes - The Difference Between Satisfaction with Care and Perception of Surgical Outcome}

Ben Z. Roitberg MD; Ying Cao ; Jingjing Shen MD

Introduction: Patient-rated outcome questionnaires may include questions about patient satisfaction with care and were criticized for being a poor tool to evaluate the performance of the surgical team. As our spine registry included separate questions regarding perceived outcome and about perceived quality of care - we have an opportunity to compare the two metrics.

Methods: 1400 patients were consecutively enrolled in a prospective spine outcome registry. assessment included ODI/NDI; VAS; SF-36; questions about satisfaction with careandperception of outcome. Data were filled by the patient, blinded to the providers. 599 patients for whom at least two years of follow up was scheduled by $3 / 8 / 2016$ were included in this analysis.

Results: Response rate was $86 \%$ at $3,85 \%$ at 6 and $82 \%$ at 12 months postoperatively. There were 303 females, 296 males; average age 58.8 years; 388 patient had lumbar and 211 - ceervical operations. Satisfaction with care and perception of outcome correlated - Pearson $r=0.67 ; p<0.0001$. Most patients felt improved and had a positive opinion of their care. Some patients had divergent opinion of care vs outcome. At 3,6 and 12 months respectively $5.5 \%, 7.2 \%$ and $9.8 \%$ were satisfied with outcome but not care, whereas $3.1 \%$, $3,3 \%$ abd 2,5\% satisfied with care but not outcome. Of 16 patients who at 3 months felt that they were same or worse after the operation, 14 dropped out by 12 months (88\%). Of those who perceived "good outcome" but "poor care" 20/28 dropped out.

Conclusion: Patients can distinguish between their own perceived outcome and the quality of their care. Perceived poor outcome leads to loss to follow up. 
8:02 - 8:04 am

\section{Factors Associated With C5 Palsy Following Cervical Spine Surgery: A Systematic Review}

Andrew S. Jack MD; Joseph Dettori; Line G. Jacques MD, MSc, FRCS(C), DABNS; Robert A. Hart; Jens Chapman MD

Introduction: $\mathrm{C} 5$ palsy (C5P) is a not uncommon postoperative complication with a reported incidence varying between $0-30 \%$. One explanation for its occurrence includes foraminal nerve root tethering. Although different risk factors have been reported, controversy about its causation and prevention persists. Inconsistent study findings contribute to the persistent ambiguity leading to an assumption of a multifactorial nature of the underlying C5P pathophysiology.

Methods: Electronic databases from inception to 2019 and references of articles were searched. Narrow inclusion criteria were applied to identify studies investigating demographic, clinical, surgical, and radiographic factors associated with postoperative C5P.

Results: Sixteen studies were included after initial screening of 122 studies. Eighty-four risk factors were analyzed; 27 in $\geq 2$ studies and 57 in single studies. The prevalence of C5P was $6.0 \%$ (range: 4.2-24.1\%) with no consistent evidence that C5P was associated with demographic, clinical, or specific surgical factors. Of the radiographic factors assessed, decreased foraminal diameter (FD) and preoperative cord rotation were identified as risk factors for C5P.

Conclusion: Although risk factors for C5P have been reported, ambiguity remains due to potentially multifactorial pathophysiology and study heterogeneity. We found FD and cord rotation to be associated with postoperative C5P occurrence in our meta-analysis. These findings support the notion that factors contributing to, and acting synergistically with foraminal stenosis and potentially nerve root tethering increase the risk of postoperative C5P. 
8:04 - 8:06 am

\section{Engineering Biomaterial for Mesenchymal Stem Cells Delivery to Regenerate Nucleus Pulposus}

\section{Lee Onn Chieng}

Introduction: Degeneration of intervertebral disc is an irreversible process, with currently no effective biological treatment. Our lab previously demonstrated robust potential of nucleus pulposus (NP) like cells for disc regeneration in a rabbit model of disc regeneration. Manipulation of environment cues with hydrogel scaffolds have been employed in tissue engineering based on their biocompatibility, elasticity, water content, and ability to permit the diffusion of nutrients. The objective of this research was to develop a novel hydrogel based scaffold for further optimized differentiation of human umbilical cord mesenchymal stem cells (MSCs) into nucleus pulposus-like cells (NPCs).

Methods: The optimal conditions for 3-D culture of human NPCs was determined by combining different polymers at various concentrations $(1.25,2.5,5$, and $10 \mathrm{w} / \mathrm{v} \%)$, which were then monitored for scaffold formation, cell dispersal, and growth. For analysis in 3-D culture, human NPCs were grown in self- assembling scaffolds (PEG-4-Acr/PEG-4-SH, and PEG-8-Acr/PEG -8-SH) for 7 days, and then treated with $2 \mu \mathrm{M}$ VP for an additional 7 days. Cell growth in the scaffolds was monitored by light microscopy. NPCs grown in 3-D selfassembling scaffolds were harvested after 14 days of culture with and without YAPi treatment as a control. Cells were harvested for RNA and immunocytochemical analysis detailed below.

Results: When cells were transplanted with 3-D PEG scaffolds, expression of all chondrogenic and NP specific markers were significantly increased at levels much higher than when cells alone were transplanted. Overall, this indicates that the 3-D PEG-4-SH/PEG-4-Acr scaffolds provided a more suitable niche for NPCs differentiation, with higher NP marker expression and extracellular matrix glycosaminoglycan production, and was more efficacious in NP regeneration ex vivo.

Conclusion: We have developed a 3D scaffolds thatimprove the maintenance and differentiation of NP like cells by manipulating its microenvironment cues which potentially accelerate its translation in human nucleus pulposus disc regeneration. 
8:06 - 8:08 am

\section{Diabetes is a Predictor of Postoperative C5 Nerve Root Palsy after Posterior Cervical Laminectomy and Fusion.}

Yaroslav J. Gelfand MD; Joshua Benton BA; Michael Longo BA; Rafael De la Garza Ramos MD; Vijay Yanamadala MD, MBA; Reza Yassari MD, MS

Introduction: C5 nerve root palsy is a well-known complication of posterior cervical laminectomy and fusion (PCLF) operations. Posterior approach, male gender and presence of OPLL, were previously implicated as potential predictors of this complication.

Methods: We retrospectively reviewed the medical records of patients who underwent PCLF from 2014-2019 at a single inner-city institution. Those who had metastatic disease to the cervical spineor infection were excluded from the study. Two groups were established as follows: patients who did not develop C5 palsy postoperatively (group 1), and those that developed C5 palsy (group 2).

Results: 92 patients satisfied the inclusion criteria. Average age was 64.6 , and $57.6 \%$ were male. Ossified posterior longitudinal ligament (OPLL) was present in $18.2 \%$ of patients and was not associated with C5 palsy. $48.9 \%$ of patients were diabetic. 13 patients (14.1\%) developed C5 nerve root palsy postoperatively. On univariate analysis diabetes $(p=0.005)$ and presence of cancer $(p=0.023)$ were the only independent predictors of C5 nerve root palsy postoperatively. On multivariate regression diabetes remained statistically significant predictor of C5 nerve root palsy (OR 8.8, 95\% Cl: 1.6-49.2, p=0.013). Hemoglobin A1C did not differ significantly between those with and without C5 palsy.

Conclusion: Diabetes is well known to result in neuropathy secondary to the development of microvascular disease. While further larger studies are warranted, we present here evidence that diabetes is likely associated with C5 nerve root palsy complication in patients undergoingPCLF. 
8:08 - 8:10 am

\section{Lumbar Intervertebral Disc mRNA Sequencing Identifies the Regulatory Pathway in Patients with Degenerative Conditions}

Mohamad Bydon MD; F. M. Moinuddin PhD; Yagiz U. Yolcu; Waseem Wahood MS; Mohammed A. Alvi MD; Anshit Goyal MBBS; Mohammed Elminawy; Amel Dudakovic; Ahmad Nassr MD; Noelle Larson; Andre van Wijnen

Introduction: Lumbar degenerative disc disease (DDD) is a multifaceted progressive condition and often accompanied by disc herniation (DH) and/or degenerative spondylolisthesis (DS). Given the high prevalence of the disease (up to $20 \%$ according to some estimates) and the high costs associated with its care, there is a need to explore novel therapies such as regenerative medicine. Exploring these novel therapies first warrants investigation of molecular pathways underlying these disorders.

Methods: Next generation RNA sequencing (RNA-seq) was conducted on mRNA isolated from 10 human nucleus pulposus (NP) samples of lumbar degenerated discs (DH and DS; $n=5$ for each tissue) and other musculoskeletal tissues (Bone, cartilage, growth plate, and muscle; $n=7$ for each tissue). Pathway and network analyses based on gene ontology (GO) terms were used to identify the biological functions of differentially expressed mRNAs.

Results: A total of 701 genes were found to be significantly upregulated in lumbar NP tissue compared to other musculoskeletal tissues. These differentially expressed mRNAs were primarily involved in DNA damage, immunity and G1/S transition of mitotic cell cycle. Interestingly, DH-specific signaling genes showed major network in chemotactic (e.g., CXCL10, CXCL11, IL1RL2 and IL6) and matrix-degrading pathway (e.g., MMP16, ADAMTSL1, 5, 8, 12, and 15), while DS-specific signaling genes were found to be those involved in cell adhesion (e.g., CDH1, EPHA1and EFNA2) and inflammatory cytokines (e.g., CD19, CXCL5, CCL24, 25 and XCL2).

Conclusion: Our findings provide new leads for therapeutic drug discovery that would permit optimization of medical or pharmacological intervention for cases of lumbar DDD. 
8:10 - 8:12 am

\section{The Current State of Knowledge of Providers Caring for Patients with Neonatal Brachial Plexus Palsy}

Brandon W. Smith MD, MSCR; Molly McNeely; Kate Chang; Lynda Jun-San Yang MD, PhD; John E. McGillicuddy MD

Introduction: Despite continued improvement in treatment options/outcomes in NBPP, there continues to be delay in referral. Additionally, a recent study demonstrated the underutilization of surgical intervention for patients with NBPP. The authors hypothesize that there is a lack of knowledge in providers caring for patients with NBPP.

Methods: A 12 question survey on NBPP diagnosis, prognosis, and management was distributed. Participants were providers from various specialties across North America. Each question was analyzed individually, and each participant was categorized into 1 of 3 groups based on performance. Statistical analysis was performed.

Results: 272 practitioners were surveyed: 169(72\%) physicians, 37(13\%) therapists, 13(5\%) advanced practice providers, and $28(10 \%)$ other. Practitioners ranged broadly in time in practice, with a significant portion $(30 \%)$ in practice for over 10 years. Overall, $29 \%$ of practitioners acknowledged comfort in diagnosing/managing NBPP. Only $25 \%$ of practitioners knew the correct timing for referral, with $10 \%$ of the respondents answer 1year which is outside the window for intervention. $81 \%$ percent of respondents incorrectly believed that $90 \%$ of NBPP cases get full spontaneous recovery.

Conclusion: This survey reveals the concerning knowledge state regarding the diagnosis and management of NBPP. The self-reported comfort level in managing NBPP is low among providers, and only 1 out of every 4 practitioners knew the ideal timing for referral. $81 \%$ of the group incorrectly thought that $90 \%$ of patients have full recovery. Initiatives should be developed to increase awareness and to improve provider education. 


\section{8:12 - 8:14 am}

\section{Comparison of Various Methods of Axillar Nerve Reconstruction in Upper Brachial Plexus Palsy}

Pavel Haninec; Libor Mencl MD, PhD

Introduction: Restoration of shoulder function is one of the main priorities of brachial plexus surgery. Neurotization, the transfer of a functional but less important donor nerve to a nonfunctional, more important recipient nerve, has recently become a leading treatment option. A variety of donor nerves have been used to reinnervate the axillary nerve with various degrees of success.

Methods: Of 1380 nerve reconstructions in 582 patients with brachial plexus injury performed by the senior author (P.H.), a subset of 152 patients (132 male and 20 female) underwent axillary nerve reconstruction using either lower subscapular nerve, triceps branch of the radial nerve, thoracodorsal nerve, long thoracic nerve, intercostal nerves or fascicle transfer from the ulnar or median nerve. The median age was 33 years, and the median time between trauma and surgery was 6 months.

Results: Successful deltoid recovery was defined with muscle strength $\mathrm{MRC}$ grade $=3$, electromyographic increase in the amplitude and duration of motor unit action potentials and by evaluation of muscle mass increase. The success rate of the lower subscapular nerve transfer, in axillary nerve reconstruction, evaluated in our study was $69.2 \%$, and was very similar effectiveness of other donors with success rate $4486 \%$.

Conclusion: The lower subscapular nerve presents valuable technique which can be added to nerve surgeons armamentarium. 
8:14 - 8:16 am

An Analysis of Medicare Reimbursement for Neurosurgeon Office Visits: 2010-2018

Maya Harrington; Jordan Pollock; Jack M. Haglin BA; Kent Richter; Naresh P. Patel MD

Introduction: Trends in outpatient office visit billing and procedural reimbursement within Medicare remain unclear within the field of neurosurgery.

Methods: The 2010 and 2018 physician/supplier files from the Centers for Medicare and Medicaid website were utilized for this study. All payments submitted by neurosurgeons (provider ID 14) were included. Trends in office visit CPT codes 99201-99205 and 99211-99215 were analyzed.

Results: Neurosurgeons billed for 1,109,979 office visits (463,611 hours) in 2010 and Medicare denied 63,704 payments (totaling $\$ 11,205,283)$. Neurosurgeons billed for $1,189,046$ office visits $(508,526$ hours) in 2018 and Medicare denied 57,048 payments (totaling $\$ 15,103,879$ ). This is a $7.1 \%$ increase in total neurosurgery office visits billed to Medicare, $9.7 \%$ increase in total office hours, and $34.8 \%$ increase in denied payment amount over 8 years. Additionally, Medicare paid $36.1 \%$ of the total neurosurgeon charge amount for all office visits in 2010, but paid $27.8 \%$ in 2018 (-8.3\%). In 2010, the most commonly denied visit was existing patient visit of 40minutes (99215, Denied 7.8\%), and in 2018 it was existing patient visit of 5-minutes (99211, Denied 7.6\%). The most frequently performed office visit by neurosurgeons for both 2010 and 2018 was existing patient visit of 15 -minutes at $32.3 \%$ and $32.4 \%$ of all office visits respectively. The 25 -minute office visits for established patients had the largest increase in billing from 2010 to 2018 (169,328 visits in 2010, 252,023 visits in 2018, $+48.8 \%)$.

Conclusion: Neurosurgeons are performing more office visits and spending more time with patients. Meanwhile, Medicare has been denying more payments and reimbursing a decreasing percentage of submitted charges for office visits. A comprehension of these trends is necessary to ensure continued, quality access to neurosurgical care in the United States. 
8:16 - 8:18

\section{Postoperative Urinary Retention (POUR) in Elective Lumbar Spinal Surgery: A Quality Improvement Project - Final Results}

Jake Jasinski DO; Doris Tong; Chad F. Claus DO; Evan J. Lytle DO; Matthew Bahoura BA; Clifford Houseman DO; Peter L. Bono D.O.; Teck M. Soo

Introduction: The incidence of postoperative urinary retention (POUR) after elective lumbar spine surgery ranges from $8 \%$ to $38 \%$. This is the final analysis from our quality improvement (QI) intervention to lower the incidence of POUR in our institution.

Methods: We conducted retrospective chart reviews for consecutive elective lumbar surgeries from 10/1/15 9/30/16 to determine pre-intervention baseline POUR. POUR risk factors and possible preventive measures were identified. Multi-disciplinary work groups developed a standardized intervention protocol: catheterization protocol, OR Foley insertion protocol, ambulation POD0, Foley removal POD1, and prophylactic Flomax for males. The primary outcome was POUR as determined by reinsertion of Foley postoperatively, failure of voiding test and discharged with Foley, or POUR as diagnosed by urology. Post-intervention data from consecutive elective lumbar surgeries were collected prospectively between 5/30/18-11/1/18. Univariate analyses and stepwise multiple logistic regression (SMLR) were used. $\mathrm{P}<0.05$ was considered significant with Bonferroni correction when applicable.

Results: We studied 277 pre-intervention and 422 post-intervention patients. Significant demographic differences between the pre- and post-intervention groups were previous history of UR ( $0 \%$ vs. $4.5 \%, p=.000)$, proportions of smokers $(28.5 \%$ vs. $21.3 \%, p=0.03)$, and duration of surgery (136 min vs. $120 \mathrm{~min}, \mathrm{p}=0.008)$. Our POUR rate and length of hospital stay decreased significantly from $6.9 \%$ to $2.6 \%(p=.007)$ and 2.94 to $2.56 \mathrm{~d}(\mathrm{p}=0.017)$ respectively. Compliance outcomes, proportions of patients returning from OR with Foley, ambulation POD0, and prophylactic Flomax use significantly improved post-intervention. SMLR demonstrated patients to be at 2.64 times higher odds of POUR pre-intervention vs. post-intervention (95\% Cl $1.2-5.7$, $\mathrm{p}=.015)$. Increasing surgery duration was associated with a statistically significant, but clinically insignificant increased likelihood of POUR (OR 1.01, p=0.02).

Conclusion: The implementation of our POUR prevention protocol significantly lowered our POUR rate and length of stay following elective lumbar surgery. 
8:18 - 8:20 am

\section{Long-term Results after Surgical or Non-surgical Treatment of Lumbar Spinal Stenosis: A Prospective Multicenter Study}

Jakob M. Burgstaller; Johann Steurer; Isaac Gravestock; Florian Brunner; Tamas Fekete; Giuseppe Pichierri; Nils H. Ulrich; Sebastian Winklhofer; Francois Porchet ; Mazda Farshad

Introduction: The optimal treatment strategy for patients with degenerative lumbar spinal stenosis (DLSS) is the subject of much debate.

Methods: Outcomes were quantified by mean values and achievement of minimal clinically important difference (MCID) scores for the Spinal Stenosis Measure (SSM) symptoms and function scales, and EQ-5D$3 \mathrm{~L}$ (quality of life) summary index (SI), from baseline to 3 years' follow-up.

Results: 601 patients met the inclusion criteria: 430 underwent surgery, 18 of them more than one year after enrollment (changers), and 171 received non-surgical treatment. At baseline, the surgical and non-surgical groups had similar values for SSM symptoms and SSM function scores, but patients in the surgical group had significantly more buttock pain and more often reported a worsening of symptoms over the three months prior to enrollment. Surgical treatment (except changers) was associated with significantly better outcomes (p0.001) than non-surgical treatment, and outcomes plateaued at 1 year follow-up in both groups. Two thirds of patients in the surgical group achieved the MCID for function, symptoms, and quality of life, compared with only about half for symptoms and even fewer for function and quality of life in the non-surgical group.

Conclusion: Surgical treatment of DLSS was associated with more favorable clinical outcomes with a sustained effect over time, compared with non-surgical treatment. 
8:20 - 8:22 am

Growth Hormone Enhances Response to Therapeutic Electrical Stimulation in Rat Sciatic Injury Model

Richard Price; Ying Yan; Nathan Birenbaum; Christopher Dibble; Matthew R. MacEwan BSE, PhD; Zack Ray

Introduction: Peripheral nerve injuries represent a major cause of debilitating sensorimotor deficits that cause significant morbidity worldwide. Despite significant advances in surgical technology, multimodal therapies are lacking. Additional multimodal therapy to improve peripheral nerve recovery are desperately needed.

Methods: Adult Lewis rats were divided into four treatment groups $(n=6)$. All rats were subjected to transection and repair injury of the right sciatic nerve. Group 1 received no additional therapy, group 2 received subcutaneous injection of $\mathrm{GH}(0.4 \mathrm{mg} / \mathrm{day})$ for 28 days, group 3 had wireless stimulator implanted at the cut and repair site of the sciatic nerve, and group 4 received wireless stimulator implantation and $\mathrm{GH}$ injection for 28 days. Both groups 3 and 4 received 1-hour therapeutic electrical stimulation $(2.5 \mathrm{~V}, 20 \mathrm{~Hz})$ for six consecutive days starting on POD 0 . All animals were sacrificed at 8 weeks for terminal testing of EMG (tibialis anterior) and immunohistochemical studies of muscle and nerve.

Results: There was no significant difference in weight among animals that received GH compared to $\mathrm{GH}$-nave groups. Rats that received GH and therapeutic electrical stimulation (group 4) had earlier recovery of EMG amplitudes than rats only receiving electrical stimulation (group 3). Additionally, the $\mathrm{GH} /$ therapeutic stimulation group had significantly higher EMG amplitudes at early timepoints.

Conclusion: In preliminary studies the addition of $\mathrm{GH}$ to therapeutic stimulations leads to earlier and more robust EMG amplitudes in rats suggesting enhanced nerve regeneration with multimodal therapy. 
8:22 - 8:24 am

\section{Comparison of Outcomes in Spinal Epidural Abscess Patients with Medical versus Surgical Initial Management}

Yaroslav J. Gelfand MD; Michael Longo BA; Zach Pennington BS; Rafael De la Garza Ramos MD; Vijay Yanamadala MD, MBA; Daniel M. Sciubba MD; Reza Yassari MD, MS

Introduction: Recently many spinal epidural abscess (SEA) patients with favorable characteristics and no neurologic deficits have been managed medically first. Up to $40 \%$ failed initial medical therapy.

Methods: Retrospective review was performed of medical records in two large institutions in underserved urban areas in the US. First, we compared patients who underwent surgery with those who were managed medically first (medical-first group vs surgery-first group); second we compared the outcomes using post-op Frankel grade and change in Frankel grade between medical-first and surgery-first groups. Univariate analysis followed by multivariate regression model was used to analyze the data.

Results: We identified a total of 103 patients. Average age was 58.2 and $57.3 \%$ were male. Out of 103 patients, 52 were managed medically initially. Of those 21 (40.4\%) failed medical management and required surgery. Those who were older and with a Frankel grade D or better (had no or mild neurologic deficit) were more likely to be managed conservatively. There was no significant difference in number of patients with good postoperative Frankel grade ( $\mathrm{D}$ or $\mathrm{E}$ ) between medical-first and surgery-first groups. In multivariate model controlling for differences between surgical and medical first groups, only pre-operative neurologic status was significant in predicting good postoperative Frankel (D and E) (OR 56.0 95\% Cl: 9.4-332.3, p<0.001).

Conclusion: Patients with SEA and no significant neurologic deficit pre-op who are attempted on medical therapy first, have no worse outcomes than those who undergo immediate surgery. Surgical group has better overall improvement in Frankel grade, but only in patients who had significant pre-operative neurologic deficit. 


\section{8:24 - 8:26 am}

\section{Novel MRI-based Score for Assessment of Bone Density in Operative Spine Patients}

Jeffrey S. Ehresman BS; Zach Pennington BS; Andrew Schilling; Daniel Lubelski MD; A. Karim Ahmed; Ethan Cottrill BS; Majid Khan; Daniel M. Sciubba MD

Introduction: Good bone quality is key in avoiding a multitude of afflictions, including osteoporotic fragility fractures and poor outcomes after spine surgery. In patients undergoing instrumented spine fusion, bone quality often dictates screw pullout strength, insertional torque, and vertebral body loading properties. While dual-energy X-ray absorptiometry (DEXA) screening is the current method of assessing bone mineral density, the majority of patients do not have DEXA measurements available before undergoing surgical instrumentation.

Methods: Using non-contrast T1-weighted magnetic resonance images (MRIs) of the lumbar spine, the novel VBQ score was calculated for each patient. DEXA T-scores of the femoral neck and total hip were obtained and werecompared to patient VBQ scores using linear regression and Pearsons correlation.

Results: Among the 68 patients included in this study, 37 were found to have osteopenia/osteoporosis (Tscore <-1.0) based on DEXA. A greater VBQ score was significantly associated with the presence of osteopenia/osteoporosis with a predictive accuracy of $81 \%$. VBQ scores correlated moderately with femoral neck T-scores, the lowest overall T-scores of each patient, and correlated fairly with total hip T-scores.

Conclusion: This is the first study to correlate the novel VBQ score obtained from MRIs with DEXA T-score. We found this score to be a significant predictor of healthy versus osteopenic/osteoporotic bone with an accuracy of $81 \%$, and found that VBQ score was moderately correlated with femoral neck and overall lowest Tscore. 


\section{8:26 - 8:28 am}

\section{Prognosticators of Return-to Work Among Employees Undergoing Lumbar Surgery: A MSSIC Study}

Mohamed Macki MD, MPH; Travis M. Hamilton MD; Hesham M. Zakaria MD; Michael Bazydlo MS; Lonni Schultz PhD; Jad Khalil; Miguelangelo Perez-Cruet; llyas Aleem MD, Msc, FRCSC; Paul Park MD; David Nerenz PhD; Jason M. Schwalb MD, FAANS, FACS; Muwaffak Abdulhak; Victor W. Chang MD

Introduction: Return-to-work may represent a surrogate marker of patient recovery after spine surgery. Return-to-work arguably affects patient satisfaction/ perception of surgical success. Surgical consent discussions usually prompt patients to ask Will I be able to go back to work? Thus, the rates and factors associated with return-to-work are worth investigating.

Methods: Michigan Spine Surgery Improvement Collaborative (MSSIC) was queried for all lumbar operations among only those patients who were employed prior to surgery. Return-to-work was assessed at 90-days, 1year, and 2-years postoperatively. Multivariable Poisson generalized estimating equation (GEE) models reported adjusted risk ratios $\left(R_{\mathrm{adj}}\right)$.

Results: Of the 3003 patients undergoing lumbar surgery, postoperative return-to-work reached $87 \%$ of 1333 patients at 90 -days, $84 \%$ of 1109 patients at 1 -year, and $83 \%$ of 561 patients at 2 -years. Following a multivariable regression at 90 -days, return-to-work statistically significantly decreased the most by $23 \%$ among African-Americans versus White-Caucasians $\left(\mathrm{RR}_{\mathrm{adj}}=0.77[0.61-0.96]\right)$ followed by $18 \%$ with fusions versus decompression surgery $\left(\mathrm{RR}_{\mathrm{adj}}=0.82[0.77-0.88]\right), 17 \%$ among high school diploma/general education development $\left(R_{\mathrm{adj}}=0.83[0.71-0.97]\right), 14 \%$ with medium/heavy physical workload $\left(R_{\mathrm{adj}}=0.86[0.81-0.92]\right)$, $13 \%$ for Medicare/ other public insurance versus private insurance (RRadj=0.87[0.80-0.96]), and $11 \%$ for every 10-unit incremental increase in postoperative Oswestry Disability Index (ODI) $\left(R_{\mathrm{adj}}=0.89[0.87-0.91]\right)$.

At 1-year, return-to-work decreased the most by $19 \%\left(R_{\mathrm{adj}}=0.81[0.68-0.98]\right)$ for Medicaid followed by $8 \%$ for Medicare/ other public insurance $\left(\mathrm{RR}_{\mathrm{adj}}=0.92[0.85-0.99]\right)$. Higher postoperative ODI and fusion surgery also decreased return-to-work by 7\% $\left(R R_{a d j}=0.93[0.91-0.95]\right)$ and 5\% $\left(R R_{a d j}=0.95[0.90-0.99]\right)$, respectively.

At 2-years, return-to-work decreased the most by $9 \%$ with previous spine surgery $\left(R R_{a d j}=0.91[0.84-0.98]\right)$ and $4 \%$ with increasing postoperative ODI $\left(\mathrm{RR}_{\mathrm{adj}}=0.96[0.94-0.98]\right)$. Smoking actually increased return-to-work by $11 \%\left(R_{\mathrm{adj}}=1.11[1.01-1.22]\right)$.

Conclusion: The rate of return-to-work among preoperatively employed patients remained above $80 \%$ up to 2 years after lumbar surgery. Socioeconomic factors, like White-Caucasians and private insurance, carried the strongest probability of return-to-work at 90-days and 1-year postoperatively. Non-fusion lumbar surgery and lower postoperative ODI also increased the chances of return-to-work at all time points. 
8:28 - 8:30 am

\section{Using Machine Learning to Predict 30-Day Readmissions after Posterior Lumbar Fusion: A NSQIP Study of 23,264 patients}

Benjamin Hopkins BS; Jonathan T. Yamaguchi BS; Hannah Weiss BA; Roxanna Garcia MD, MS, MPH; Tyler R. Koski MD; Nader S. Dahdaleh MD

Introduction: Unplanned preventable hospital readmissions within 30 days are a great burden to patients and the healthcare system. With an estimated $\$ 41.3$ billion spent yearly, reduction of such readmission rates is of utmost importance. With the widespread adoption of big data and machine learning, analytical tools can help clinicians understand these complex relationships and find predictive factors that can be generalized to future patients.

Methods: We analyzed the distribution of the National Surgical Quality Improvement Program posterior lumbar fusions from 2011-2016 using machine learning techniques in order to create a model predictive of hospital readmissions. A Deep Neural Network (DNN) was trained using 177 unique input variables. The model was trained and tested using cross-validation, in which the data were randomly partitioned into training $(n=17,448$, $75 \%)$ and testing $(n=5,816,25 \%)$ datasets. In training, the 17,448 training patients were fed through a series of 9 layers each with varying degrees of forward and backward communicating nodes (neurons).

Results: Average and median PPV were $67.1 \%$ and $77.1 \%$, respectively. Average and median NPV were $96.6 \%$ and $96.9 \%$ respectively. Average and median AUC for our model was 0.812 and 0.810 , respectively. The top five most heavily weighted inputs were return to operating room, septic shock, superficial surgical site infection, and being on a ventilator for $>48$ hours.

Conclusion: Machine learning and artificial intelligence are powerful tools with the ability to improve understanding of predictive metrics in clinical spine surgery. Our model was able predict patients that would not require readmissions. Similarly, the majority of predicted readmissions (up to 60\%) were predicted by our model while retaining a $0 \%$ false positive rate. Such findings suggest possible need for reevaluation of the current Hospital Readmission Reduction Program penalties in spine surgery. 
8:30 - 8:32 am

\section{Mesenchymal Stem Cell-Seeded High-Density Collagen Gel for Annular Repair: 16-Week Results From In Vivo Sheep Models}

Sertac Kirnaz MD; Christoph Wipplinger; Stephen Sloan; Franziska A. Schmidt MD; Lawrence J. Bonassar PhD; Roger Hartl MD

Introduction: Our group has previously demonstrated in vivo annulus fibrosus repair in animal models using an acellular, riboflavin crosslinked, high-density collagen (HDC) gel.

Methods: We performed an anterolateral, retroperitoneal pre-psoas approach to access the IVDs L1-6 in a total of 10 skeletally mature Finn sheep. IVDs were randomized into four groups: 1) intact, 2) injured via $3 \times 10 \mathrm{~mm}$ annulotomy and removal of $200 \mathrm{mg}$ of NP, 3) injury and acellular HDC gel patch for AF repair, 4) injury and MSC-seeded HDC gel patch for AF repair. At 16 weeks postoperatively, sheep were sacrificed and underwent post-mortem 3T-MRI scans as well as gross anatomical and histological evaluation. Disc height index (DHI) analysis and modified Pfirrmann grading (PG) were performed on each segment using MR images.

Results: Intact control discs showed no degeneration and had an average PG of 1/8 while injured, untreated discs had significant degeneration with an average PG of 5/8. Discs receiving MSC-seeded HDC gel patch for AF repair showed fewer signs of degeneration than the injury only group and equivalent amounts of degeneration as the PG to the acellular HDC gel receiving group. DHI confirmed the trends seen in the PG, where injured discs lost $32 \%$ of the intact disc height, the acellular group lost $21 \%$ of intact disc height, and the MSC-seeded HDC gel patch preserved $89 \%$ of the intact disc height.

Conclusion: PG and DHI results demonstrated that HDC gel patch with/without MSCs were able to prevent disc degeneration better than no treatment at all. MSC-seeded HDC gel group was better at preserving DHI than the acellular group. 
8:32 - 8:34 am

\section{Prophylactic Closed-Incisional Negative Pressure Therapy In Spine Surgery: A Multi-center, Observational Study}

Kyle Mueller MD; Matthew R. D'Antuono BS; Nirali Patel; Gnel Pivazyan; Edward Aulisi; Karen Evans MD; Nathan Nair MD

Introduction: Surgical site infections increase healthcare cost and result in diminished quality in spine surgery. Closed-incisional negative pressure therapy (ciNPT) stabilizes the surgical closure and prevents shear and motion around the incision. This has been used to facilitate healing of challenging wounds; however, its prophylactic use in spine surgery is not well studied.

Methods: We performed a prospective, multi-center, observational study in which we prophylactically placed either a ciNPT or standard sterile dressing over the closed incision at the time of surgery in matched cohorts. Durotomy was considered a contraindication. Baseline patient characteristics and wound complications up to 60 days following surgery were recorded.

Results: 212 patients were included in the study. 104 patients received ciNPT and 108 received standard dressing. There were statistically more patients with a higher BMI (32.4 vs. 28.9, p=0.001), diabetic (44 vs. 24 , $p<0.001)$ and $>3$ levels (90 vs. $50, p<0.001)$ in the ciNPT group compared with standard surgical dressing. The overall SSIR was $13.9 \%$ (39/281). The SSIR in the ciNPT was less than with standard surgical dressing $(5.8 \%$ vs $18.6 \%, p=0.005)$.

Conclusion: Prophylactic closed incisional negative pressure therapy results in a significant reduction in surgical site infections following spine surgery. This investigation supports the need for further study in select high risk groups, such as spinal oncology and deformity. 


\section{8:34 - 8:36 am}

\section{The Impact of Preoperative Depression on Patient Satisfaction with Spine Surgeons in the Outpatient Setting}

Hammad A. Khan BS; Nicholas M. Rabah BS; Jay M. Levin BA; Robert Winkelman BS; Thomas E. Mroz MD; Michael P. Steinmetz MD

Introduction: The Clinician and Group Assessment of Healthcare Providers and Systems (CG-CAHPS) survey is used to measure patient experience in the outpatient setting. CG-CAHPS scores are made publicly available and may be used by prospective patients when selecting spine surgeons or by hospital systems in physician incentive programs. While preoperative depression has been shown to correlate with worse patientreported outcomes following spine surgery and lower patient satisfaction in the inpatient setting, no studies have examined its impact on patient experience with their spine surgeons in the outpatient setting.

Methods: Patients who underwent elective spine surgery between 2009-2017 and completed a CG-CAHPS survey during postoperative follow-up were included in this study. Data was collected on patient demographics, Patient Health Questionnaire (PHQ-9) scores, and Patient-Reported Outcome Measurement Information System Physical Functioning (PROMIS-PF) scores. Patients with preoperative PHQ-9 scores of greater than or equal to 10 (moderate to severe depression) were included in the depressed cohort. Survey responses on several dimensions of the CG-CAHPS survey were compared between depressed and non-depressed patients using chi-square tests. "Top-box" survey responses were considered indicators of high patient satisfaction.

Results: Our cohort included 419 patients, of which 72 met criteria for preoperative depression. On average, depressed patients were younger $(p<0.001)$ and had lower self-reported physical functioning $(p<0.001)$ compared to non-depressed patients. $73.9 \%$ of depressed patients provided a "top-box" Overall Provider Rating compared to $90 \%$ of non-depressed patients $(p<0.001)$. Further, depressed patients were more likely to feel that their spine surgeon did not listen to them carefully $(p=0.003)$ and less likely to recommend their office to other patients $(p=0.018)$.

Conclusion: This study suggests that preoperative depression is associated with lower patient satisfaction with their spine surgeon in the outpatient setting. 
8:36 - 8:38 am

\title{
The Role of Neuromonitoring in Anterior Lumbar Interbody Fusion
}

\author{
Anan Shtewe; Kartik Kesavabhotla; Nader S. Dahdaleh MD; Tyler R. Koski MD
}

Introduction: The anterior lumbar interbody fusion is a common procedure performed for a number of conditions. What is unclear is whether or not an anterior approach to the spine necessitates the use of intraoperative neuromonitoring. At this time, the use of neuromonitoring is surgeon-dependent. we report our experience and study the incidence of neuromonitoring changes seen during ALIFs.

Methods: Retrospective analysis of prospectively collected data at a single institution since 2010-2019, of all patients undergoing surgery for anterior lumbar interbody fusion (ALIF) of either one or two levels. The primary outcome was neurological injury or any action required by the senior surgeon as a response to neuromonitoring changes. Our secondary aim has been to assess the cost associated with ALIF neuromonitoring at a single institution.

Results: 120 consecutive patients (62 males and 58 females) with average age of 58.8 years. Of these cases, 39 patients underwent a staged posterior lumbar fusion for deformity correction. Operative indications included symptomatic spondylolisthesis, radiculopathy, and neurogenic claudication. No neuromonitoring alerts or changes were noted intraoperatively within this cohort. No patients had SSEP/MEP changes that required action by the senior surgeon. Neuromonitoring is billed hourly and associated with costs ranging between $\$ 1000$ and $\$ 2000$ per case, with average ALIF duration between 2-4 hours.

Conclusion: Neuromonitoring is a very important and vital tool when preforming certain spine surgeries like deformity correction, lateral approach to the lumbar spine and many more. Our data over the last 10 years for single surgeon includes 120 patients that had one or two-level ALIF, shows that monitoring doesnt have any additive value to the procedure and doesnt affect the normal course of the instrumentation. This is important since it may significantly reduce the time and the cost of such surgery. 
Spine Summit Meeting Abstracts, May 2021

8:38 - 8:40 am

\section{Bariatric Surgery Diminishes Spinal Symptoms in a Morbidly Obese Population: A 2-Year Survivorship Analysis}

Peter G. Passias MD; Avery Brown; Haddy Alas; Cole Bortz BA; Katherine Pierce; Waleed Ahmad; Sara Naessig; Erik Wang; Hamid Hassanzadeh; Lawal Labaran; Varun Puvanesarajah BS; Aaron J. Buckland MBBS, FRACS; Michael Gerling

Introduction: Bariatric surgery for morbid obesity helps to address common comorbidity burdens, including decreasing rates of myocardial infarction and stroke.

Methods: Retrospective analysis of the prospectively collected (NYSID) years 2004-2013. Patient linkage codes allow identification of multiple and return inpatient stays within the time-frame analyzed (720 days). Inclusion criteria were bariatrics surgery patients with one or more visits prior to and after bariatric procedure(excluding pts with $<30$ days $\mathrm{f} / \mathrm{u}$, spine surgery, or new post-bariatric spine pathology) for one of the following common cervical or lumbar spinal diagnoses, queried with ICD-9CM codes: herniation, stenosis, spondylosis, disc degeneration, and spondylolisthesis. Time from bariatric surgery until the patient's respective spinal diagnosis was no longer present was considered resolution of spinal symptoms. Kaplan-Meier survivorship curves assessed rates of resolution within each spinal diagnosis cohort.

Results: 4,351 bariatric surgery pts with a pre-op spinal diagnosis by ICD-9 were analyzed. Lumbar pts: 1,049 had stenosis, 774 spondylosis, 648 degeneration, 249 spondylolisthesis, 72 disc herniation. Cervical pts: 581 disc herniation, 376 had stenosis, 366 spondylosis, 236 degeneration. Cumulative resolution rates at 90-day, 180-day, 360-day, and 720-day f/u were as follows: lumbar stenosis (48\%,67.6\%,79\%,91\%), lumbar herniation $(61 \%, 77 \%, 86 \%, 93 \%)$, lumbar spondylosis $(47 \%, 65 \%, 80 \%, 93 \%)$, lumbar spondylolisthesis $(37 \%, 58 \%, 70 \%, 87 \%)$, lumbar degeneration $(37 \%, 56 \%, 72 \%, 86 \%)$. By cervical region: cervical stenosis $(48 \%, 70 \%, 84 \%, 94 \%)$, cervical herniation $(39 \%, 58 \%, 74 \%, 87 \%)$, cervical spondylosis $(46 \%, 70 \%, 83 \%, 94 \%)$, cervical degeneration $(44 \%, 64 \%, 78 \%, 89 \%)$. Lumbar herniation pts saw significantly higher $90 \mathrm{~d}$-resolution than cervical herniation $\mathrm{pts}(\mathrm{p}<0.001)$. Cervical vs lumbar degeneration resolution rates did not differ $@ 90 d(p=0.058)$, but did @180d(p=0.034). Cervical and lumbar stenosis resolution was similar @90d \& 180d, but cervical showed greater resolution by $1 Y(p=0.036)$.

Conclusion: Over $50 \%$ of bariatric patients diagnosed with a cervical or lumbar pathology before weight-loss surgery no longer sought inpatient care for their respective spinal diagnosis by 180 days post-op. Lumbar herniation had significantly higher resolution than cervical herniation by $90 \mathrm{~d}$, whereas cervical degeneration and stenosis resolved at higher rates than corresponding lumbar pathologies by $180 \mathrm{~d}$ and $1 \mathrm{Y}$ f/u, respectively. 
8:40 - 8:42 am

\title{
The Effect of Obesity on Adjacent Segment Revision Surgery after TLIF for Spondylolisthesis
}

\author{
Ping G. Duan MD, PhD; Praveen V. Mummaneni MD; Sigurd Berven MD; Rory R. Mayer; Bo Li; Dean Chou
} MD

Introduction: Revision surgery for adjacent segment degeneration (ASD) is known to occur after spinal fusion.

Methods: Patients who underwent single-level TLIF for spondylolisthesis from 2006 to 2016 were retrospectively analyzed. Inclusion criteria were: minimum 2-year follow up, single level TLIF, clinical and radiographic follow-up. Patient demographic data were collected, and analyses of spinopelvic parameters were performed. The patients were divided into two groups based upon pelvic incidence (PI)-lumbar lordosis (LL) mismatch as mismatched (PI - LL $\geq 10^{\circ}$ ) and balanced $\left(\mathrm{PI}-\mathrm{LL}<10^{\circ}\right)$. Within the two groups, the patients were further classified by body mass index $\left(\mathrm{BMI}, \mathrm{kg} / \mathrm{m}^{2}\right)(<30, \geq 30)$ or $(<30, \geq 30<35, \geq 35)$. Patients were then evaluated at last follow up for revision surgery for ASD, matched by BMI and PI-LL mismatch.

Results: A total of 190 patients met inclusion criteria (72 males and 118 females, mean age 59.57 years). The average follow-up was 40.21 months . In total, 24 patients (12.6\%) underwent revision surgery for ASD, with 82 patients mismatched and 108 patients balanced. In the mismatched group (82/190), there were 6/34 revisions (17.6\%) in $\mathrm{BMI} \geq 30$ patients and $1 / 48$ revision $(2.1 \%)$ in $\mathrm{BMI}<30$ patients. Within the mismatched group, revisions occurred at a rate of: $1 / 12(8.3 \%)$ in $\mathrm{BMI} \geq 35$ patients, $5 / 22(22.7 \%)$ in $\mathrm{BMI} \geq 30<35$ patients, and $1 / 48(2.1 \%)$ in $\mathrm{BMI}<30$ patients. Statistical differences were seen between patients with $\mathrm{BMI} \geq 30$ and $\mathrm{BMI}<30(P=0.013)$ and between patients with $\mathrm{BMI} \geq 35,30 \leq \mathrm{BMI}<35$, and $\mathrm{BMI}<30(P=0.014)$. However, in the balanced PI-LL group (108/190 patients), there was no difference in revision surgery rates for ASD among the patients with different BMls $(P>0.05)$.

Conclusion: In patients who have PI-LL mismatch, obesity may be associated with an increased risk of revision surgery for ASD after TLIF, but in patients with balanced PI-LL, no difference was observed. 
8:42 - 8:44 am

\section{Utilization of Posterior Lumbar Fusion Varies By Yearly Quarter: Insights from The National Inpatient Sample}

Waseem Wahood; Alex Alexander; Mohammed A. Alvi MD; Anshit Goyal MBBS; Elizabeth Habberman; Mohamad Bydon MD

Introduction: Elective lumbar fusion is a commonly employed procedure for degenerative spine disease. However, with rising healthcare costs, elective neurosurgical procedures are under scrutiny for insurance coverage.

Methods: We queried the Healthcare Cost and Utilization Project-National Inpatient Sample (HCUP-NIS) between January 1, 2012 and December 31, 2014 for adult patients diagnosed with lumbar disc degenerative disease (DDD) from 2012-2014. Patients undergoing posterior lumbar fusion (PLF) were identified using ICD-9 codes. Outcomes of interest included total hospital charges, utilization and frequency of PLF. Patients were grouped based on primary insurance type.

Results: A total of 262,065 patients diagnosed with Lumbar DDD between 2012 and 2014 were identified. Of these, 67,833 (25.9\%) underwent a PLF. Variance-weighted least squares analysis revealed that the overall trend in utilization of lumbar fusion for patients with Medicare was statistically different from those with private insurance (difference in slope: $0.0347, p=0.008$ ), as well as between Medicaid and privately-insured patients (difference in slope: $0.084, p=0.002$ ). Compared to privately insured patients, all other patients had a lower probability of undergoing PLF, with those uninsured showing the lowest rate (Medicare: OR 0.86, 95\% $\mathrm{Cl}$ : 0.83-0.88, p<0.001; Medicaid: OR 0.69, 95\% Cl: 0.66-0.71, p<0.001; Uninsured: OR 0.50, 95\% Cl: 0.45-0.56, $\mathrm{p}<0.001)$. Overall, average utilization of lumbar fusion was significantly higher in the $4^{\text {th }}$ quarter, compared to $1^{\text {st }}$ quarter (OR 1.054, 95\% Cl: 1.03-1.08, p<0.001).

Conclusion: These results indicate that utilization of PLF increases in the fourth quarter each year, especially for patients with private insurance. This phenomenon may be due to reduced out of pocket expenditure for healthcare later in the year. These findings may ensure timely access to treatment for patients with Lumbar DDD. 


\section{8:44 - 8:46 am}

\section{Mild and Severe Obesity Reduce Effectiveness of Lumbar Fusions: One Year Patient-Reported Outcomes in 8,171 Patients}

Graham Mulvaney; Matthew J. McGirt MD; Vincent Rossi MD; David Peters MD; Olivia Rice; Joshua Patt; Deborah Pfortmiller; Anthony L. Asher MD, FACS

Introduction: BMI is a well-known risk factor for surgical morbidity and complication in lumbar surgery. However, the effect of mild to severe obesity on the overalleffectiveness of lumbar fusion in improving painrelated disability independent of surgical complicationsis less understood.

Methods: We analyzed a prospective, nationwide, patient-reported quality-of-life registry representing 75 hospital systems (QOD)for patients who received elective 1-3level lumbar fusion for stenosis and spondylolisthesis. Patients who experienced surgical complications were excluded. A stepwise multivariate regression model was usedto assess factors independently associated with 12moODI score,pre-op to $12 \mathrm{moODI}$ change score, and achievement of MCID (14.3-point ODlimprovement).

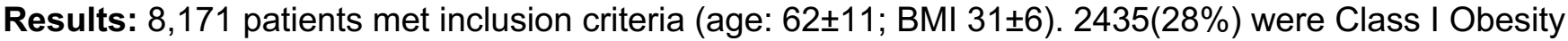
(BMI:30-35), 1328(15\%) Class $2(35-40)$, and 760(9\%) Class $3(\geq 40)$. In multivariate analysis, increasing BMI was independently associated with worse $12 \mathrm{mo}$ ODI $(t=8.005, p<.001)$ and decreased likelihood of achieving MCID $(\mathrm{OR}=.977, \mathrm{p}<.001)$. One year after surgery, mean ODI, ODI change score, and \% achieving MCID worsened with Class 1 , Class 2 , and Class 3 vs non-obese cohorts $(p<0.001)$ in stepwise fashion.

Conclusion: One year after elective 1-3 level lumbar fusion, increasing BMI was independently associated with worse pain-related disability and decreased effectiveness of surgery despite lack of surgical complications. While effectiveness of surgery was significantly reduced in Class III Obesity (BMI $\geq 40.0)$, BMI 30-35 was also associated with reduced surgical effectiveness. BMI as low as 30-35 may represent a modifiable risk factor for reduced effectiveness/value of lumbar fusion. 
8:46 - 8:48 am

\title{
Medicare Reimbursement For Services Billed By Neurosurgeons From 2010-2018
}

\author{
Jordan Pollock; Kent Richter; Jack M. Haglin BA; Naresh P. Patel MD
}

Introduction: The complexity and lack of transparency in Medicare billing and reimbursement is well known to medical providers.

Methods: The 2010 and 2018 physician/supplier files from the Centers for Medicare and Medicaid website were accessed. Payments were sorted by Provider ID, and all payments being submitted by neurosurgeons (provider ID 14) were included and analyzed in this study.

Results: In 2010 and 2018, neurosurgeons billed Medicare for $\$ 3,441,742,892(4,003,438$ services) and $\$ 4,513,528,985(3,919,454$ services) respectively. Although number of services billed by neurosurgeons has decreased from 2010 to 2018, Medicare has increasingly denied payment for services, which totaled 732,678 denied payments $(\$ 446,956,533,18 \%$ of submitted charges) in 2010 and 833,872 denied payments ( $\$ 580,742,056,21 \%$ of submitted charges) in 2018 . In 2010 , the average submitted charge by neurosurgeons was $\$ 859.70$, the Medicare allowed charge was $\$ 166.73$ (19\% of billed amount), and the Medicare payment amount was $\$ 130.65$ (15\% of billed amount). In 2018 , these same values were $\$ 1,151.57, \$ 206.79$ (18\% of billed amount), and $\$ 158.78$ (14\% of billed amount).

Conclusion: From 2010 to 2018, the number of services billed to Medicare by neurosurgeons has decreased. Additionally, Medicare has denied an increasing amount of billed services and is paying a smaller portion of the billed amount. Complex billing practices might be resulting in more denied payments and rising charges by neurosurgeons for services. As Medicare is one of the largest healthcare insurers in the United States, relevant trends in billing practices are important to understand. 
8:48 - 8:50 am

\section{Comparing the Predictive Ability of DRGs with Comorbidity Measures in ALIF}

Sean N. Neifert BS; Rebecca Baron; John M. Caridi MD; Ian T. McNeill MD; Travis Ladner MD; Jonathan Gal

Introduction: Hospital reimbursements are processed via Diagnosis Related Groups (DRG) that accounts for patient-level complexity with a 3-tiered system. However, no investigations compare this system to commonlyused comorbidity measures in patients undergoing anterior lumbar interbody fusion (ALIF).

Methods: Patients undergoing ALIF at a single institution from 2008 to 2016 were reviewed. The tiered DRG system (no complication/comorbidity, complication/comorbidity, and major complication/comorbidity) as well as the $\mathrm{ECl}, \mathrm{CCl}$, and ASA Class were noted for each patient. Characteristics and outcomes between patient cohorts based on these metrics were compared, and predictive cost modeling was performed

Results: The increasing DRG tiers correlated with ASA Class $(r=0.16), E C l(r=0.16)$ and $\mathrm{CCl}(r=0.14)$. Higher ASA Class $(p<0.0001), E C l(p<0.0001)$, and $\mathrm{CCl}(p<0.0001)$ correlated with increased age, and increasing ASA Class $(p=0.02)$ and $E C l(p=0.04)$ correlated with increased segments fused. Rising DRG tier also correlated with increased blood loss $(p<0.0001)$ and longer operative time $(p<0.0001)$. Increasing DRG tier $(p<0.0001)$, ASA Class ( $p<0.0001), E C l(p<0.0001)$ and $\mathrm{CCl}(p=0.03)$ all correlated with longer length-of-stay. Predictive cost modeling, showed the DRG tier system to have the strongest predictive ability (Rsquared=0.10) followed by ASA Class (R-squared=0.03), ECl (R-squared=0.02), and CCl (R-squared=0.01).

Conclusion: While other metrics may have better predictive ability for other outcomes, the DRG system remains optimal for prediction of cost. Further research is needed to examine situations in which other predictive measures should be utilized to optimize patient outcomes. 
8:50 - 8:52 am

\section{Adipose Tissue-Derived Stromal Vascular Fraction Cells With Neither Culture Expansion Nor Priming in Spinal Fusion}

Alexander Perdomo-Pantoja MD; Christina Holmes; Alexandra Rindone; Ethan Cottrill BS; Wataru Ishida MD; Maritza N. Taylor; Colson Tomberlin; Sheng-fu L. Lo MD; Warren Grayson; Timothy F. Witham BS, MD

Introduction: The stromal vascular fraction of adipose tissue can be readily isolated from the lipoaspirate of patients and can be an alternative therapeutic mesenchymal stromal cell source. Contrary toex vivoexpanded adipose-derived MSCs, little research has been made using freshly isolated adipose tissue-derived stromal vascular fraction (A-SVF) cells.

Methods: SVF of adipose tissue was isolated and combined with Vitoss (Stryker) bone graft substitute for subsequent transplantation. Posterolateral spinal fusion surgery at L4-L5 was performed on rats divided into 3 experimental groups: [1] Vitoss only (VO group or Control); [2] Vitoss + 2.5x106 A-SVF cells/side; and, [3] Vitoss + 2.5x106 BMA cells/side. Fusion was evaluated eight weeks post-surgery via manual palpation, microcomputed tomography $(\mu \mathrm{CT})$ imaging, histology, and immunohistochemistry.

Results: $\mu C T$ imaging analyses showed that fusion volumes and $\mu C T$ fusion scores in the A-SVF group were significantly higher than in the VO group $(22.07 \pm 2.01 \mathrm{~mm} 3$ vs. $14.9 \pm 1.3 \mathrm{~mm} 3$, respectively, $p=.01$, and $1.58 \pm 0.14$ vs. $1.08 \pm 0.08$, respectively, $p=.03$ ); however, they were not significantly different between the $A$ SVF group and the BMA group (19.54 $\pm 1.57 \mathrm{~mm} 3$ and $1.33 \pm 0.14, p>.05)$. The average manual palpation score was highest in the A-SVF and BMA compared to VO group.

In histology, A-SVF and BMA groups displayed higher osteoid deposition, number of osteoblasts, and neovascularization compared to VO group, and expressed endothelial marker CD31 across the entire area of the fusion mass.

Conclusion: In our rat model, A-SVF cells achieved vascularized bone formation and yielded a comparable fusion mass volume and radiographic rate of fusion to BMA cells when combined with a clinical-grade bone graft substitute. These results suggest the feasibility of using freshly isolated SVF in spinal fusion procedures. 


\section{8:52 - 8:54 am}

\section{Predictors of Unemployed Patients Returning to Work After Lumbar Surgery}

Mohamed Macki MD, MPH; Travis M. Hamilton MD; Michael Bazydlo MS; Lonni Schultz PhD; Hesham M. Zakaria MD; Jad Khalil; Miguelangelo Perez-Cruet; Ilyas Aleem MD, Msc, FRCSC; Paul Park MD; David Nerenz PhD; Jason M. Schwalb MD, FAANS, FACS; Muwaffak Abdulhak MD, FRCS; Victor W. Chang MD

Introduction: Degenerative and deformity lumbar diseases requiring surgerycanrender patient unable to return-to-work, which has profoundly affects not only patient-reported outcomes but also larger societal implications.

Methods: The prospectively collected database for the Michigan Spine Surgery Improvement Collaborative (MSSIC) was queried for all lumbar operations. Preoperative patient surveys selected only those patients who were intending to return to work who were otherwise unemployed secondary to low back problems. A multivariable analysis first examined demographic or medical variables (age, gender, race, ambulatory status, symptom duration >1yr, PHQ-2 depression score, or baseline ODI) followed by behavioral, socioeconomic, and surgical factors (daily opiod use $>6$ mon, public versus private insurance, at least some college, workers' compensation, fusion versus decompression alone, and multi-level versus single-level operations).

Results: Among patients unemployed preoperatively, rates of postoperative return-to-work reached $34 \%$ after 90 days $(\mathrm{N}=1328), 49 \%$ after 1 -year $(\mathrm{N}=926)$, and $53 \%$ after 2 -years $(\mathrm{N}=577)$. Following a multivariable regression, there were no significant associations between demographic or medical variables with return-towork at any time point. However, among socioeconomic facors, the odds of return-to-work at 90days decreased by $55 \%$ with preoperative daily opioid use $>6$ months $\left(\mathrm{OR}_{\mathrm{adj}}=0.45[0.29-0.68]\right), 36 \%$ with public insurance $\left(\mathrm{OR}_{\mathrm{adj}}=0.64[0.44-0.94]\right), 49 \%$ with workers' compensation $\left(\mathrm{OR}_{\mathrm{adj}}=0.51[0.31-0.86]\right)$, and $52 \%$ with fusion surgery $\left(\mathrm{OR}_{\mathrm{adj}}=0.48[0.35-0.67]\right)$. At 1-year, only preoperative daily opioid use $>6$ months $\left(O R_{a d j}=0.47[0.30-0.75]\right)$ and public insurance $\left(O R_{a d j}=0.67[0.50-0.90]\right)$ were negatively associated with returnto-work. There were no significant associations at 2-years.

Conclusion: The rate of return to work for unemployed patients increase to up to $53 \%$ after 2-years after elective lumbar surgery.Demographic and medical factors do not appear associated with return to work, although chronic opiate use, and public insurance appear to play a role. Therefore, return to work seems to be influenced more by socioeconomic factors as opposed to medical. 
8:54 - 8:56 am

\section{Preoperatively Predicting Discharge Disposition After Lumbar Laminectomy Using a Machine-Learning Classification Model}

Robert Winkelman BS; Rod Nault; Ghaith Habboub MD; Matthew M. Grabowski MD; Vikram Chakravarthy MD; Sebastian Salas-Vega PhD; Jason Savage; Thomas E. Mroz MD

Introduction: Prolonged length of stay (LOS) can be a major driver of cost following elective spine surgery. Previously, we have identified patients who required post-acute care services (PACS [i.e. homecare or discharge to a facility]) after lumbar laminectomy surgery at our institution have longer LOS when surgery is scheduled late in the week (i.e. Thursday, Friday) compared to earlier in the week. Therefore, improving the surgical teams understanding of patients post-acute care needs prior to surgery may provide opportunities to better coordinate care in order to mitigate prolonged LOS.

Methods: A retrospective chart review was performed on 962 patients who underwent lumbar laminectomy surgery at a single healthcare system from 3/1/2016 through 2/28/2019. Patients discharge disposition was classified according to the need for PACS (Yes vs. No). Several machine-learning models to predict patients need for PACS were developed using the following variables: patient characteristics (age, gender, Elixhauser comorbidities), health questionnaire data (PROMIS Global Health), and operative characteristics (surgeon, number of levels).

Results: The final cohort included 253 patients (26.3\%) who required PACS. The final model selected was a boosted tree model with a classification accuracy of $73 \%$. The models sensitivity and specificity for detecting the need for PACS in a validation cohort was $70 \%$ and $74 \%$, respectively. The most important variables as determined by loss from feature dropout were the presence of diabetes, surgical levels, and PROMIS-GH responses for preoperative physical activity, pain, and general health.

Conclusion: Our study demonstrates that machine-learning classification models can assist in preoperatively identifying patients who will require PACS after surgery. By understanding patients post-discharge needs prior to surgery, the surgical team can improve preoperative patient counseling and/or optimize surgical scheduling to potentially avoid unnecessary prolonged LOS. 
8:56 - 8:58 am

\title{
Antibiotic Stewardship and Single-Dose Antibiotic Prophylaxis: A Word of Caution for Spine Surgery
}

\author{
Allen L. Ho MD; Martin N. Stienen MD; John K. Ratliff MD, FACS
}

Introduction: Multidrug-Resistant Organism and Clostridium difficile infections (MDRO/CDI) have been identified as a hospital safety metric by the Centers for Medicare and Medicaid Services (CMS). Many institutions have created antibiotic stewardship programs to ensure that guidelines limiting use of postoperative antibiotics are enforced. These guidelines often recommend only a single preoperative dose of antibiotics for the entire perioperative period for spine procedures.

Methods: This was a prospective study of the all patients receiving spinal surgery in a single academic neurosurgery department in the six months prior to and following implementation of a single-dose perioperative antibiotic prophylaxis policy. Before this, all patients were receiving multi-dose post-operative antibiotics. All patients were reviewed for antibiotic use, surgical drain usage, surgical site infections (SSIs), SSI treatment via antibiotic therapy or return to OR, and MDRO/CDIs. All patients had at least 3 months follow-up.

Results: The first six months of this single-dose protocol yielded a greater than twofold increase in patients with SSIs that had to return to the operating room for surgical washout and wound revision compared to the six months prior $(0.73 \%$ to $2.4 \%, P=0.17)$. There were no clostridium difficile infections or concurrent increases in multi-drug resistant infections during this period. Review of the literature regarding single-dose protocols for spine surgery found evidence to be based primarily on non-instrumented spine procedures or comparisons to no-antibiotic protocols.

Conclusion: National guidelines targeting safety and quality metrics are often generalized and based on nonspecialty specific or outdated evidence. Administrative efforts to target MDRO/CDI at the institutional level with antibiotic-limiting protocols need to be met with appropriate caution in spinal surgery patients. 
8:58 - 9:00 am

\section{Vascularized Scapular Grafts: A Novel Approach for Spinal Fusion}

Terence Verla MD, MPH; Aditya Vedantam MD; Matthew Davis; Edward Reece; Michael R. Raber MD; David S. Xu MD; Alexander E. Ropper MD

Introduction: Spinal fusion can be challenging to obtain in patients with complex spinal pathology. Medial scapular vascularized bone grafts (S-VBGs) are a novel approach to supplement cervicothoracic arthrodesis in patients at high risk for failed spinal fusion.

Methods: $79 \mathrm{M}$ presented to our institution with a 9-month history of cervical spondylotic myelopathy, chin-onchest deformity, 30-year smoking history and previous neck radiation for stage- $V$ pharyngeal cancer. He underwent C2-T4 decompression and fusion accompanied by S-VBG placement. Scapular bone graft harvest and rotation were performed safely, and the patient experienced excellent clinical recovery with in tact sensory and motor function.

Results: The S-VBG is harvested in the prone position. The medial scapula is cut longitudinally from either the superior or inferior angle using a high-speed drill or an osteotome, staying lateral to the descending branch of the transverse cervical artery. The subscapularis muscle is then divided inferior to superior, again stopping before damaging the descending branch. The completely mobilized graft remains on the rhomboid and trapezius muscles and is supplied by unnamed vessels coming through Sharpeys fibers. Bleeding edges of bone can be used intraoperatively to confirm that the graft remains vascularized. The S-VBG is tunneled underneath the paraspinous muscles and secured in the recipient site and then the entire fusion construct is covered with bilateral paraspinous muscle flaps.

Conclusion: The S-VBG is a novel surgical technique that leverages the advantages of vascularized structural allograft without the increased operative time and morbidity associated with free tissue transfer. This graft has been demonstrated to supplement complex cervicothoracic arthrodesis cases, and we also believe that with additional study, scapula-based vascularized bone grafts could serve as useful adjuncts in other cervicothoracic surgeries. 
9:00 - 9:02 am

\section{The Impact of Surgical Chronology on Outcomes of Patients Receiving Lumbar Spine and Lower Extremity Joint Surgeries}

Karam Atli MS; Gabriel A. Smith MD; Konrad Knusel; Edward C. Benzel MD; Ajit A. Krishnaney MD, FAANS; Michael P. Steinmetz MD; Thomas Mroz

Introduction: The aging population and obesity epidemic indicate more patients with lumbar spine pathology will likely develop lower extremity joint (LEJ) disease (hip and/or knee). These patients may have co-existing lower back and extremity pain, the major source of which (spine vs. LEJ) is difficult to determine. There remains a lack of outcomes data of patients who receive surgeries for both an LEJ and the lumbar spine based on chronology of surgery type.

Methods: Retrospective chart review was performed of patients undergoing lumbar surgery between 20082015 and receiving hip/knee surgery within three years prior/following lumbar surgery at a single institution. Pre- and post-operative outcomes were assessed using the EuroQol five dimensions questionnaire (EQ5D) and Pain Disability Questionnaire (PDQ).

Results: 670 patients were included. Patients receiving lumbar surgery first were 4.75 times more likely to reach the minimal important difference in PDQ. Patients receiving LEJ surgery first had a higher mean Charlson Comorbidity Index. Male patients were $37 \%$ less likely to be readmitted within 90 days of either procedure.

Conclusion: Postoperatively, patients receiving lumbar surgery before LEJ surgery had greater improvement than patients receiving LEJ surgery before lumbar surgery. These findings suggest that in patients with a coexisting need for lumbar and LEJ surgery, greater consideration should be given to providing lumbar surgery before LEJ surgery, and warrants further large-scale prospective investigation todetermine the cause and generalizability of these outcomes results. 
9:02 - 9:04 am

\section{The Utility of Neuromonitoring on Posterior Lumbar Fusions - Analysis of the National Inpatient Sample Database}

William Steele MD; Ryan Austerman; Sean Barber; Suraj Sulhan

Introduction: IOM technology is used to potentially detect neurologic injury in real time during fusion procedures, theoretically reducing the rate of new postoperative neurological deficits. However, the routine use of IOM in elective posterolateral lumbar fusions (PLF) remains controversial.

Methods: The National Inpatient Sample (NIS) was analyzed for the years 2012-2015 to identify patients undergoing elective PLF.Discharges with ICD-9 procedure codes for posterior lumbar fusion (81.07 and 81.08 ) were identified for patients 18 years or older. Included patients were then divided into those with and without an ICD-9 procedure code for neuromonitoring (ICD-9 CM 00.94). Patient demographics were analyzed. The three primary outcomes analyzed in the monitoring and non-monitoring patient sub-populations were length of stay, total charge of hospitalization, and development of neurological complications.

Results: IOM use in elective PLFs was found to have increased from $14.6 \%$ in the year 2012 to $19.3 \%$ in 2015 , which is an overall increase of $1.2 \%$ per year. The total charge in hospitalization cost was also found for all patients who received IOM to have increased from $\$ 129,384.72$ in 2012 to $\$ 146,427.79$ in 2015. Cost of stay of the non-IOM patients was $\$ 108,503.52$ in 2012 and $\$ 121,898.88$ in 2015. Overall, the total charge of hospitalization was $11 \%$ greater in the IOM group when compared to those patients that did not have IOM $(95 \% \mathrm{Cl}, 11-12 \%, \mathrm{p}<0.001)$. IOM did not have a statistically significant impact on the likelihood of developing a neurological complication when adjusting for potential confounders.

Conclusion: The results of this analysis call into question the routine use of intraoperative neuromonitoring for simple posterior first time lumbar fusions for degenerative spine disease. 
9:04 - 9:06 am

\section{Three-Dimensional Printing of Titanium Without Bone Graft Outperforms PEEK + Autologous Iliac Crest Bone Graft}

Jesus Pino; Rosa Villar; Antonio Cantalapiedra; Fernando Munoz; Ramiro Couceiro; Jeffrey L. Gum MD; Andrew Pugely; Joseph Laratta

Introduction: There have been significant advancements in three-dimensional titanium printing and implant surface technology (3D-Ti), which may lead to improved cellular response, proliferation, and differentiation. However, the actual ability of these structural improvements to affect the in vivo fusion cascade has not been clearly defined.

Methods: Eight PEEK and eight 3D-Ti implants were randomized to anterior lumbar interbody fusions at L2-3 or L4-5 in a sheep model. The PEEK cages were implanted with autologous ICBG. CT scans were performed at 4-weeks. CT, microCT and histology were performed at sacrifice (8-weeks). The robustness of fusion was graded A-D; where $A$ is no fusion, $B$ is fusion spot-welding to the cage, $C$ is fusion through the cage, and $D$ is fusion outside of the cage.

Results: At both 4 and 8-weeks, there was no CT evidence of fusion (type-A) in PEEK + ICBG implants (0\%). Histologic analysis at sacrifice revealed substantial osteolysis and cellular delamination surrounding the PEEK cage with unincorporated/floating ICBG fragments. At 4-weeks in the 3D-Ti group without graft, there were 6/8 (75\%) type-B fusions and 2/8 (25\%) robust fusions (type-D). On CT and microCT at 8-weeks, the entire 3D-Ti group (100\%) showed evidence of through and outside of the cage (type D).

Conclusion: In an in vivo sheep interbody fusion model, implants created with 3D-Ti and surface technology without bone graft outperform PEEK + autologous ICBG with regards to radiographic and histologic fusion. The three-dimensional titanium technology with biomechanically similar characteristics to trabecular bone allows for more rapid, robust fusion in an animal model and may minimize the need for bone grafting and osteoinductive compounds. 
9:06 - 9:08 am

\section{Surgical Management of Anterior Cervical Osteophytes Causing Dysphagia}

Joshua Kolz; Mohammed A. Alvi MD; Atiq Bhatti; Mohamad Bydon MD; Yagiz U. Yolcu MD; Ahmad Nassr MD; Bradford L. Currier MD; Brett Freedman

Introduction: Anterior cervical osteophytes are usually asymptomatic, however when large enough they can cause dysphagia. There is currently a paucity of work examining outcomes of anterior cervical osteophyte resection for dysphagia.

Methods: We identified 19 patients who underwent an anterior cervical osteophyte resection for a diagnosis of dysphagia between 1999 and 2017. There were 17 (89\%) males, a mean age of 71 years, and mean BMI of 27 $\mathrm{kg} / \mathrm{m}^{2}$. The most common spinal level operated on was C4. Preoperative work-up included video fluoroscopic swallowing exam (100\%), CT scan (74\%), MRI (73\%,) cervical spine x-rays (37\%), EMG (5\%), and evaluation by Gastroenterology (47\%), Physical Medicine and Rehabilitation/Speech (37\%), and Neurology (11\%).

Results: Following anterior cervical osteophyte resection for a diagnosis of dysphagia 15 of the 19 patients $(79 \%)$ had a significant improvement in their dysphagia. Six patients underwent cervical fusion in conjunction with the osteophyte resection. There was only one pseudoarthrosis that was lost to follow-up after 2 years. There was a single case of osteophyte regrowth at the index level along with the proximal level. Mean operative time was 178 minutes and mean length of stay was 2.5 days after surgery.

Conclusion: Anterior cervical osteophyte resection improves swallowing function in the majority of patients with symptomatic osteophytes. Prior to surgery, patients should undergo thorough swallow evaluation to ensure their anterior cervical osteophytes are the primary cause of dysphagia. Additionally, there is a relatively high complication rate $(32 \%)$, which highlights the need for a multidisciplinary approach to the workup and treatment of these patients. 
9:08 - 9:10 am

\section{The Effect of Estimated Blood Loss on Lumbar Spinal Fusion Outcomes}

Mohamed Macki MD, MPH; Travis M. Hamilton MD; Michael Bazydlo MS; Lonni Schultz PhD; Hesham M. Zakaria MD; Jad Khalil; Miguelangelo Perez-Cruet; Ilyas Aleem MD, Msc, FRCSC; Paul Park MD; David Nerenz PhD; Jason M. Schwalb MD, FAANS, FACS; Muwaffak Abdulhak MD, FRCS; Victor W. Chang MD

Introduction: Minimization of EBL is a core element of ERAS. Utilizing the Michigan Spine Surgery Improvement Collaborative (MSSIC), our hypothesis: increased EBL would be associated with negative outcomes.

Methods: All lumbar fusion patients within MSSIC were eligible for this study divided into three cohorts: 1 level, 2-level, and $\geq 3$-level fusions. EBL was analyzed as a categorical variable: $<250 \mathrm{~mL}, 250-500 \mathrm{~mL}$, and $>500 \mathrm{~mL}$. Multivariable logistical regressions controlled for potential confounders: surgical approach, interbody, anticoagulant use, and comorbidities. ALIF and LLIFs were treated as factors that would minimize EBL, while PLIF and TLIF would increase EBL. Multivariable logistical regressions produced adjusted odds ratios $\left(\mathrm{OR}_{\mathrm{adj}}\right)$.

Results: Of the total 16497 patients, 8164 patients with 1-level fusions, 4763 patients with 2-level fusions, and 3570 patients with $\geq 3$-level fusions had an average EBL of $198.99 \pm 214.00 \mathrm{~mL}, 323.15 \pm 301.75 \mathrm{~mL}$, and $499.30 \pm 478.86 \mathrm{~mL}$, respectively.

The $\geq 3$-level fusions demonstrated the statistically significantly highest rates of any complication, LOS $\geq 3 \mathrm{~d}$, and non-home discharge.

On multivariate analysis for all cohorts, EBL $<250 \mathrm{~mL}$ was the reference group. For 1-level fusion, EBL of 250$500 \mathrm{~mL}$ and $>500 \mathrm{~mL}$ was associated with longer LOS $\left(\mathrm{OR}_{\mathrm{adj}}=1.68[1.40-2.01]\right.$ and $O \mathrm{R}_{\mathrm{adj}}=1.81[1.33-2.45]$, respectively) and non-home discharge $\left(O R_{a d j}=1.50[1.16-1.94]\right.$ and $O R_{a d j}=1.72[1.14-2.58]$, respectively). For 2level fusions, EBL of $250-500 \mathrm{~mL}$ and $>500 \mathrm{~mL}$ was associated with longer LOS (OR $\mathrm{adj}=1.61[1.32-1.94]$ and $\mathrm{OR}_{\mathrm{adj}}=1.70$ [1.31-2.19], respectively), while $E B L>500 \mathrm{~mL}$ was associated with non-home discharge $\left(O R_{\mathrm{adj}}=1.45\right.$ [1.04-2.02]). For $\geq 3$-level fusions, EBL of $250-500 \mathrm{~mL}$ and $>500 \mathrm{~mL}$ was associated with higher rates of complication $\left(\mathrm{OR}_{\mathrm{adj}}=1.52[1.05-2.19]\right.$ and $\mathrm{OR}_{\mathrm{adj}}=2.23[1.51-3.29]$, respectively) and increased LOS (OR $\mathrm{Odj}_{\mathrm{ad}}=1.34$ [1.07-1.69] and $\mathrm{OR}_{\mathrm{adj}}=2.35$ [1.82-3.05], respectively), and $\mathrm{EBL}>500 \mathrm{~mL}$ was associated with non-home discharge $\left(\mathrm{OR}_{\mathrm{adj}}=1.72[1.29-2.28]\right)$.

Conclusion: Our findings illustrate associations between increased EBL and negative outcomes in elective lumbar surgery on multivariate analysis. While association does not denote causation, our findings do suggest that minimizing EBL may be an effective way to decrease odds of post-operative complication, reduce LOS, and decrease the odds of non-home discharge. 
9:10 - 9:12 am

\section{Optimization of a Disc Degeneration Model in the Rat Lumbar Spine}

Stephen Stephan; Juliane Glaeser; Wafa Tawackoli BS; Derek Ju; Jae Hyuk Yang; Linda Kanim; Khosrowdad Salehi; Victoria Yu; Zhanna Khnkoyan; Zachary Napier; Hyun Bae; Dmitriy Sheyn;

Introduction: Disc puncture injury rat models are used to study the progression of IVD degeneration, which depend highly on the needle diameter and mode of insertion. This study aims to create a reliable model to mimic early disc degeneration.

Methods: 45 Sprague Dawley rats underwent anterior lumbar disc surgery. Rats were injured with needle sizes of $18 G(n=10), 21 G(n=11), 23 G(n=12)$, and sham surgery $(n=12)$. MRI analysis was performed presurgery, 4-, and 8-weeks post-surgery. Biobehavioral tests (Randall-Selitto, von Frey) were performed presurgery, 3- and 6-weeks post-surgery. Post-sacrifice at week 8, qPCR was used to detect regulation of MMP3, aggrecan, CCN2 and collagen I/II levels. HE staining was performed.

Results: T1- and T2-weighted analysis showed a reduction of disc size and NP hydration in 18G needletreated discs at weeks 4 and 8. Correlation analysis demonstrated reduced T1 and NP high intensity area with increasing needle diameters. qPCR detected upregulation of MMP3 in the 18G and $21 \mathrm{G}$ groups vs uninjured, and in $21 \mathrm{G}$ vs $23 \mathrm{G}$. Aggrecan was downregulated in all injured IVDs. Collagen I and CCN2 levels increased in $21 \mathrm{G}$ vs uninjured and in $21 \mathrm{G}$ vs $23 \mathrm{G}$. Collagen II was decreased in $21 \mathrm{G}$ vs uninjured. Histology showed mild NP destruction after injury with 23G, a more severe destruction with the $21 \mathrm{G}$ and a very severe destruction of the NP and the NP/AF interface with the $18 \mathrm{G}$ needle. Biobehavioral testing resulted in a decreased neuropathic pain withdrawal threshold and decreased pain sensitization threshold in rats injured with a $18 \mathrm{G}$ needle.

Conclusion: Our results show a correlation between needle diameters used for the rat disc puncture and the extent of disc degeneration. Puncture with $18 \mathrm{G}$ results in most severe disc degeneration and reduced pain thresholds. Use of $21 \mathrm{G}$ induced more consistent expression of IVD degeneration biomarkers. 
9:12 - 9:14 am

\section{Inhibitory Effects of Vancomycin on Rat Bone Marrow-Derived Mesenchymal Stem Cell Viability and Differentiation}

Kari Hanson; Carly Isder; Kristen Shogren; Anthony L. Mikula MD; Michael J. Yaszemski; Benjamin D. Elder $\mathrm{MD}, \mathrm{PhD}$

Introduction: Intraoperative vancomycin powder can have detrimental effects on cells at higher doses in vitro, resulting in cell death, inhibited osteoblast proliferation, and lower fusion rates. Bone marrow-derived mesenchymal stromal cells (BMSCs) are a source of osteogenesis in spinal surgery. However, it is unknown how the antibiotic affects these cells viability and differentiation.

Methods: Rat BMSCs were isolated from femurs of immature female rats. Half of the cultures were given additional osteogenic factors, including $50 \mu \mathrm{g} / \mathrm{mL}$ l-ascorbic acid, $10 \mathrm{mM} \beta$-glycerol phosphate, and $0.1 \mu \mathrm{M}$ dexamethasone. Vancomycin was added to culture medium at concentrations of $0,0.04,0.4$, and $4 \mathrm{mg} / \mathrm{mL}$. Differentiation was determined by alkaline phosphatase activity (4 days post-treatment) and alizarin red staining for mineralization (14 days post-treatment). MTS activity at both time points was measured to determine cell viability..

Results: Cell viability was decreased at a high concentration of vancomycin $(4 \mathrm{mg} / \mathrm{mL})$, down to $40-60 \%$ of the control, 14 days post treatment in both the MBSC with osteogenic factors and those without. Cell differentiation at four days was not impacted by vancomycin at any dose, as evident by the alkaline phosphatase activity. However, alizarian red staining decreased 14 days post treatment with increasing vancomycin dosages, indicating a decrease in cell differentiation.

Conclusion: The inhibitory effect of vancomycin on spinal fusion may result from both reduced BMSC viability as well as impairment of osteogenic differentiation in a dose-dependent fashion. 


\section{9:14 - 9:16 am}

\section{Factors Associated With C5 Palsy Following Cervical Spine Surgery: A Systematic Review}

Andrew S. Jack MD; Joseph Dettori; Line G. Jacques MD, MSc, FRCS(C), DABNS; Robert A. Hart; Jens Chapman MD

Introduction: $\mathrm{C} 5$ palsy (C5P) is a not uncommon postoperative complication with a reported incidence varying between $0-30 \%$. One explanation for its occurrence includes foraminal nerve root tethering. Although different risk factors have been reported, controversy about its causation and prevention persists. Inconsistent study findings contribute to the persistent ambiguity leading to an assumption of a multifactorial nature of the underlying C5P pathophysiology.

Methods: Electronic databases from inception to 2019 and references of articles were searched. Narrow inclusion criteria were applied to identify studies investigating demographic, clinical, surgical, and radiographic factors associated with postoperative C5P.

Results: Sixteen studies were included after initial screening of 122 studies. Eighty-four risk factors were analyzed; 27 in $\geq 2$ studies and 57 in single studies. The prevalence of C5P was $6.0 \%$ (range: $4.2-24.1 \%$ ) with no consistent evidence that C5P was associated with demographic, clinical, or specific surgical factors. Of the radiographic factors assessed, decreased foraminal diameter (FD) and preoperative cord rotation were identified as risk factors for C5P.

Conclusion: Although risk factors for C5P have been reported, ambiguity remains due to potentially multifactorial pathophysiology and study heterogeneity. We found FD and cord rotation to be associated with postoperative C5P occurrence in our meta-analysis. These findings support the notion that factors contributing to, and acting synergistically with foraminal stenosis and potentially nerve root tethering increase the risk of postoperative C5P. 
9:16 - 9:18 am

\section{Serial Electrical Stimulation Improves Functional Recovery in Sciatic Nerve Transection and Isograft Repair in Rats}

Arman Odabas; Nathan Birenbaum; Ying Yan; Wilson Z. Ray MD; Matthew R. MacEwan BSE, PhD

Introduction: Peripheral nerve injuries represent one of the most common causes of sensorimotor deficits, yet functional recovery is frequently limited. Brief intraoperative electrical stimulation $(1 \mathrm{hr}, 20 \mathrm{~Hz})$ has been shown to improve recovery following PNI but has not been examined alongside interposition nerve grafting. Wireless implantable peripheral nerve stimulators enable investigation of serial electrical stimulation for nerve healing.

Methods: Male Lewis rats $(n=24)$ underwent transection of the sciatic nerve $5 \mathrm{~mm}$ proximal to the trifurcation, repair with a $20 \mathrm{~mm}$ reversed isograft, and implantation of a wireless nerve stimulator. Animals received either no stimulation $(n=8)$, or therapeutic electrical stimulation ( $1 \mathrm{hr}, 20 \mathrm{~Hz}, 2.5 \mathrm{~V})$ for 6 days $(n=8)$ or 12 days $(n=8)$. Functional recovery was assessed biweekly for 12 weeks by wireless EMG recordings in the tibialis anterior and gastrocnemius, and at 14 weeks by wired EMG and evoked force measurement in the extensor digitorum longus.

Results: Rats receiving 12 days of stimulation produce significantly greater maximum tetanic force in the EDL than rats receiving no stimulation. Both the 6 day and 12 day groups demonstrate significantly greater weightadjusted force production compared to the control. Although the mean force values of the 6 day and 12 day group are similar, the variance of the 12 day group is smaller. Mean terminal EMG values are greater in stimulated groups than the control, but the difference is not statistically significant.

Conclusion: Serial multi-day therapeutic stimulation improved functional recovery over brief intra-operative electrical stimulation in the setting of PNI repaired with a $20 \mathrm{~mm}$ isograft. Wireless implantable stimulators may offer custom stimulation regimens following nerve surgery. 
9:18 - 9:20 am

\title{
Distal Wrist Crease vs Traditional Longitudinal Incision for Open Carpal Tunnel Release
}

\author{
Robert Y. North MD; Caroline Hadley MD; James Bayley MD; Bruce L. Ehni MD
}

Introduction: Carpal tunnel release is one of the most common peripheral nerve surgeries. An assortment of techniques to perform the procedure have been described including open and endoscopic approaches. Endoscopic approaches using a transverse distal wrist crease incision (T-DWC) have been associated with decreased post-operative pain and earlier return to work compared to traditional longitudinal palmar incisions (L-P). Several authors have described adaptations of a distal wrist crease incision to perform open carpal tunnel release but literature comparing open release via distal wrist crease and palmar incisions is lacking.

Methods: A single center, multiple surgeon retrospective analysis of outcomes for open carpal tunnel release was performed. Clinical records for 102 consecutive carpal tunnel release operations with $>1$ year of follow up were reviewed (56 for distal wrist crease and 46 for longitudinal palmar). Comparison between distal wrist crease and longitudinal palmar incision was made for clinical outcome, complications, and need for revision surgery. Clinical outcome was scored on a scale of 1 to 4 as follows: 4) Complete resolution of symptoms 3) Significant improvement in symptoms 2) Minimal or no improvement in symptoms 1) Worsening of symptoms.

Results: Clinical records for 102 consecutive carpal tunnel release operations with $>1$ year of follow up were reviewed ( 56 for distal wrist crease and 46 for longitudinal palmar). Clinical outcomes and need for revision for the two incisions were not significantly different. Mean outcome 2.95 (T-DWC) vs $2.78(L-P)(p=0.16)$ and revision rate of $3 / 56$ (T-DWC) vs 4/46 (L-P). Longitudinal palmar incision was associated with higher overall complication rate $(p<0.05)$ and wound dehiscence $(p<0.05)$.

Conclusion: We provide evidence that open carpal tunnel release with a distal wrist crease incision compares favorably to traditional longitudinal palmar incisions with similar outcome and lower risk for complication. 
9:20 - 9:22 am

Brachial Plexus Nerve Root Avulsion Resulting in Delayed Cervical Spinal Cord Herniation

Andrew S. Jack MD; Wyatt L. Ramey MD; Jens Chapman MD; Line G. Jacques MD, MSc, FRCS(C), DABNS; Praveen V. Mummaneni MD; Carter Gerard MD

Introduction: Spinal cord herniation ( $\mathrm{SCH}$ ) typically occurs spontaneously in the thoracic spine with few case reports of cervical SCH as a delayed complication of traumatic brachial plexus avulsion injury (BPAI).

Methods: Electronic databases from inception to September 1, 2019 and article references were searched. Inclusion criteria were applied to identify studies of BPAl-associated $\mathrm{SCH}$. Chart review was completed detailing two recently encountered cases.

Results: Five studies were included after initial screening (173 studies). Seven previous cases have been described, in addition to our 2. Patients have presented in a delayed fashion (range: 2-17, mean 9-years) after the inciting trauma, and been young males (range: 18-41, mean 32.5 years-old). BPAI-SCH patients have typically presented with diffuse myelopathy or unilateral pyramidal symptoms.

\section{Cases:}

A 37-year-old man presented with progressive leg weakness 2-years after traumatic C8-T1 root avulsions. Magnetic resonance imaging (MRI) showed known C8-T1 nerve avulsions with new SCH in a T1 pseudomeningocele. A C7-T1 costotransversectomy and C4-T4 instrumented fusion was completed allowing $\mathrm{SCH}$ reduction and patch-graft dural repair without adhesiolysis. At last follow-up, the patients weakness had resolved.

A 32-year-old man presented with progressive arm numbness and signs of myelopathy 9-years after C8-T1 root avulsions. MRI showed known root avulsions, and new SCH with extensive/compressive pseudomeningocoele formation. A C7 transpedicular approach with C5-T1 instrumented fusion was completed for dural repair. The pseudomeningocoele was drained and adhesiolysis required to reduce the $\mathrm{SCH}$ for patchgraft repair. At last follow-up, the patients weakness was improving, though numbness persisted.

Conclusion: $\mathrm{SCH}$ is a rare cause of delayed neurological deterioration after BPAI with few case reports describing its occurrence. We present two cases treated successfully and epidemiologically detail this rare entity after literature review. 
9:22 - 9:24 am

\section{Resident Peripheral Nerve Surgery Exposure and Nerve Repair Technical Ability Assessment}

Andrew S. Jack MD; Beata Durcanova; Line G. Jacques MD, MSc, FRCS(C), DABNS

Introduction: Identifying peripheral nerve surgery (PNS) competencies is crucial to ensure adequate resident training exposure. No systematic evaluation currently exits for technical aspects of neurosurgical training in the US, and only recently has a Competency by Design (CBD) curriculum been implemented in Canada.

Methods: Self-reported competency and exposure were evaluated by questionnaire completion at a large, academic, US neurosurgical center, as well as across Canada. Exposure and competency were correlated with procedure-based skills from three PNC using small (2-3mm), cadaveric specimens: direct-nerve (DS), connector-assisted (CA), and connector-only (CO) repair. Variables collected included: time-to-completion, sutures required, and nerve-handling from video-analysis, blinded visual-analog-grading by 3 judges, and training level. ANOVA/2-way ANOVA (parametric) and Kruskal-Wallis/Mann-Whitney (non-parametric) analyses with post-hoc testing were completed. Statistical significance was set at $P<0.05$.

Results: Training level and PNS exposure were significantly correlated $(P<0.01)$; senior residents report more exposure to cubital-tunnel release $(P<0.01)$, brachial-plexus surgery $(P=0.01)$, direct-nerve-repair $(P=0.03)$, and nerve-transfer $(\mathrm{P}=0.02)$.

No difference was observed between training level and PNC grading $(p=0.41)$, although a between-group difference was seen for the type of PNC: DS and CA (median quality for both: fair) repairs scored better than $\mathrm{CO}$ (median: poor) $(\mathrm{p}=0.02$ and $\mathrm{p}<0.01$, respectively).

A discrepancy was observed between trainee self-reported PNS competency and PNS exposure that increased upon training level stratification.

Conclusion: Despite more exposure and a higher perceived PNS-related competency in senior residents, no difference was seen between senior/junior residents in PNC quality. A discrepancy in PNS-case exposure and perceived competency exists. This information will provide insight into the direction of PNS training, and its role in the implementation of a CBD curriculum. 
9:24 - 9:26 am

\title{
Neural Degeneration Underlies Cancer Cachexia Induced Respiratory Failure
}

\author{
Daryl Fields; Andrew Judge; David Fuller; Gordon Mitchell
}

Introduction: Cancer cachexia is an insidious process characterized by progressive motor deficits; including diaphragm weakness and eventual respiratory failure. Current therapies promoting recovery of atrophied muscle fibers have been ineffective at preserving life.

Methods: Using whole body plethysmography we assess breathing responses in mice inoculated with C26 adenocarcinoma cells. Mice were exposed to a sequence of gas mixtures: 1) air; 2) hypoxia $\left.\left(11 \% \mathrm{O}_{2}\right) ; 3\right)$ hypercapnia $\left(7 \% \mathrm{CO}_{2}\right)$; and 4$)$ combined hypercapnia/hypoxia (i.e. maximal chemoreflex response). We also tested the neural respiratory network directly using ligated phrenic nerve recordings.

Results: Cachectic mice demonstrated a significant attenuation of the hypoxic tidal volume $(0.26 \mathrm{~mL} \pm 0.01 \mathrm{~mL}$ vs $0.30 \mathrm{~mL} \pm 0.01 \mathrm{~mL} ; p<0.05)$, breathing frequency $(317 \pm 10 \mathrm{bpm}$ vs $344 \pm 6 \mathrm{bpm} ; p<0.05)$ and phrenic nerve $(29.5 \pm 2.6 \%$ vs $78.8 \pm 11.8 \% ; p<0.05)$ responses. On the other hand, the much larger hypercapnic tidal volume $(0.46 \pm 0.01 \mathrm{~mL}$ vs $0.46 \pm 0.01 \mathrm{~mL} ; \mathrm{p}>0.05)$, breathing frequency $(392 \pm 5 \mathrm{bpm}$ vs $408 \pm 5 \mathrm{bpm} ; \mathrm{p}>0.05)$ and phrenic nerve $(93.1 \pm 8.8 \%$ vs $111.1 \pm 13.2 \% ; p>0.05)$ responses were not affected. Further, the concurrent hypercapnia/hypoxia tidal volume $(0.45 \pm 0.01 \mathrm{~mL}$ vs $0.45 \pm 0.01 \mathrm{~mL} ; \mathrm{p}>0.05)$, breathing frequency $(395 \pm 7 \mathrm{bpm}$ vs $400 \pm 3 b p m ; p>0.05)$, and phrenic nerve $(106.8 \pm 7.1 \%$ vs $147.5 \pm 38.8 \% ; p>0.05)$ responses were not different between $\mathrm{C} 26$ cachectic and control mice.

Conclusion: Cancer cachexia hypoxic breathing deficits are demonstrated within whole body studiesand ligated phrenic nerves, thus reflectinging disruptions in the hypoxic chemoafferent neural network (vs muscle atrophy). This is the first study identifyinga neural etiologyto cancer cachexia respiratory failure. This insight may guide future management and treatment strategies for patients with incurable cancers. 
9:26 - 9:28 am

\section{The F2RaD Score: A Novel Prediction Score and Calculator Tool to Identify Patients at Risk of Postoperative C5 Palsy}

Daniel Lubelski MD; Zach Pennington BS; James Feghali MD; Allan J. Belzberg BSc, MD, FRCS(C); Ali Bydon MD; Daniel M. Sciubba MD

Introduction: Post-operative C5 palsy is a debilitating complication following posterior cervical decompression. A simple clinical risk score predicting the occurrence of C5 palsy remains to be developed.

Methods: We retrospectively reviewed all patients who underwent posterior cervical decompression between January 2007 and December 2017. Data was randomly split into training(70\%) and validation(30\%) datasets. Multivariable analysis was performed to construct the model from the training dataset. Model performance was assessed using receiver operating curves (ROC) on the training and validation datasets. A scoring system was developed and a web-based calculator was deployed.

Results: 415 patients were included, of which 65 (16\%) developed C5 palsy. The optimal model consisted of: mean C4/5foraminal diameter, pre-operative C5radiculopathy, anddexterity loss. The ROC yielded an area under the curve of 0.757 and 0.706 in the training and validation datasets, respectively. Every characteristic was worth 1 point except the lowest quartile of mean C4/5 foraminal diameter, which was worth 2 points, and the factors were summarized by the acronym F2RaD. The median predicted probability of C5 palsy increased from $2 \%$ in patients with a score of 0 to $70 \%$ in patients with a score of 4 . The calculator can be accessed onhttps://jhuspine2.shinyapps.io/FRADscore/.

Conclusion: This study yielded a simplified scoring system and clinical calculator that predicts the occurrence of C5 palsy. Modifying the surgical plan in high-risk patients can possibly improve outcomes. 
9:28 - 9:30 am

\section{Preoperative Exercise History: A Possible Risk Factor for Spinal Fusion Patients}

Rami Elsabeh; John M. Abrahams

Introduction: Spine patients receiving preoperative (preop) exercise therapy interventions have shown a quicker recovery and decreased hospital stay in several studies. To date, no reports in the literature have examined the association between spine patients' self-reported preop exercise histories and outcomes.

Methods: This study was a medical record review. Patients receiving lumbar, cervical or thoracic fusion between 2013 and 2018 who had exercise history and return-to-work (RTW) data were eligible. A total of 338 patients were included. Average follow-up was 6 months. Patients were stratified into five cohorts based on their self-reported preop exercise frequencies. The exercise frequency groups were as follows: Never (16\%), Rarely (35\%), Monthly (1\%), Weekly (32\%), and Daily (16\%).

Results: Patients who never exercised preop had the lowest RTW rate among all groups at 59\% (33/56). The highest RTW rate of $81 \%$ (43/53) belonged to patients who exercised daily, and those who exercised weekly had the second highest rate of $78 \%(85 / 109)$. Chi-squared tests among all groups resulted in a $p$ value of 0.051. Further Odds Ratio testing revealed that, compared to patients who never exercised, patients who exercised daily were 2.9 times more likely to return to work $(p=0.012)$ and patients who exercised weekly were 2.5 times more likely to return to work $(p=0.016)$.

Conclusion: Spinal fusion patients who had a history of exercising daily or weekly were more likely to return to work when compared to patients who had a history of never exercising. 
Sunday, March 8, 2020

Spinal Deformity

Abstract Breakout Session

7:30 - 7:32 am

Full Correction or Limited Fusion in Adult Scoliosis Surgery: Differences in Pain Alleviation and Morbidity

Minghao Wang MD, PhD; Rory R. Mayer MD; Gregory W.J. Hawryluk MD, PhD, FRCSC; Darryl Lau; Ratnesh N. Mehra DO; Dean Chou

Introduction: Full surgical correction of adult spinal deformity carries associated risks and morbidity. Decompression and limited fusion or decompression alone may, however, ultimately worsen the deformity and require revision surgery.

Methods: A retrospective review of adult spinal deformity patients treated by the senior surgeon from 2008 to 2016 was performed. Inclusion criteria were: first-time surgery, pre-op LL-PI mismatch $<30^{\circ}$, thoracic kyphosis $<60^{\circ}$, thoracolumbar kyphosis $<10^{\circ}$, coronal Cobb angle $<80^{\circ}$, and minimum 2-year follow-up. We assessed peri-operative metrics such as operative time, intra-operative blood loss and length of stay. We also recorded complications as well as re-operation rates. Patients reported quality of life outcomes including visual analog pain scale (VAS) scores for back and leg as well as Oswestry Disability Index (ODI).

Results: A total of 90 patients met inclusion criteria. Sixteen patients underwent full deformity correction, 58 underwent limited decompression and fusion, and 16 underwent decompression alone. Full deformity correction was associated with greater length of stay $(p=0.461)$, greater blood loss $(p=0.032)$ and greater operative time $(p<0.0001)$. Full deformity correction was associated with an increased rate of complications $(p<0.05$, Table 1) and re-operation $(p<0.05)$. There was no significant difference between VAS for leg pain and ODI scores among the 3 groups in terms of improvement: however, full scoliosis correction significantly alleviate back pain more than the other groups, indicated by VAS change (full correction: $5.3 \pm 1.8$, limited fusion: $0.75 \pm 3.6$, decompression alone: $1.0 \pm 5.4, p<0.05)$..

Conclusion: We found that although complete adult spinal deformity correction is associated with the greatest morbidity, there was also the greatest back pain improvement. However, limited decompression and fusion in highly selected patients primarily with leg pain may give comparable reduction in ODI and VAS leg pain scores compared to full correction. 
7:32 - 7:34 am

\section{Utilization of Thoracic and Lumbar Three-Column vs. Posterior Column Osteotomy in the Medicare Population (2008-2018)}

Timothy Chryssikos MD, PhD; Joshua R. Olexa BA; Madeleine Smith; Harry M. Mushlin MD; Nathan L. Pratt MD; Gary T. Schwartzbauer MD, PhD; Kenneth M. Crandall MD; Charles A. Sansur MD, MHSc

Introduction: Three-column osteotomy (TCO) and posterior column osteotomy (PCO) can improve spinal deformity in the elderly.

Methods: A Medicare database was queried for annual frequency and charge-to-frequency ratio (CTFR) between 2008-2018 for CPT 22206 (TCO thoracic, 1-level), 22207 (TCO lumbar, 1-level), and 22208 (TCO, additional level). 22212 (PCO thoracic, 1-level), 22214 (PCO lumbar, 1-level) and 22216 (PCO, additional level) were evaluated for comparison.

Results: For 22206, frequency increased from 71 to 219 between 2008-2018 (+208\%). 2010 (+39\%) and 2014 $(+26 \%)$ saw the largest increases. The annual change in utilization ranged from $-7 \%$ to $+2 \%$ from $2015-2018$. For 22207, utilization increased from 248 to 817 between 2008-2018 (+229\%). The largest utilization was in 2016 (828), and the largest annual increases occurred in 2009 (+45\%) and 2010 (+44\%). For 22208, frequency was 167 in 2008, peaked at 428 in 2012, and declined to 214 in 2018 . For 22212 and 22214, utilization increased from 368 to $1,403(+281 \%)$ and from 1,842 to 6,698 (+264\%) between 2008-2018, respectively. For 22216, utilization increased from 2,734 to 11,186 between $2008-2018(+309 \%)$. CTF ratio for TCO codes 22206, 22207, and 22208 increased by $2.6 \%, 1.7 \%$, and $0.8 \%$ per year on average, respectively. CTF ratio for PCO codes 22212, 22214, and 22216 increased by $1.9 \%, 0.9 \%$, and $0.5 \%$, respectively.

Conclusion: In the Medicare population, 1-level thoracic and lumbar TCO have risen since 2008 but plateaued since 2014. Additional level TCO rose from 2008-2012 but has since declined. From 2008 on frequency increased steadily for all PCO codes. The recent plateau of 1-level TCO and decline in additional level TCO against a continued rise in all PCO codes may reflect satisfaction with partial sagittal correction in the elderly, use of alternative techniques, or efforts to minimize morbidity. Minimal changes in all CTF ratios imply no pecuniary explanation for the observed trends. 
7:34 - 7:36 am

\section{Predictors of Early versus Late Distal Junctional Kyphosis Following Posterior Cervical Fusion}

Nida Fatima; John H. Shin MD

Introduction: Distal Junctional Kyphosis (DJK) is a significant complication following posterior cervical fusion (PCF) and predisposes to an increased risk of neurological injury and surgical revision.

Methods: DJK was defined as -- evidence of <-10 - from the end of fusion construct (either ending at C7 or crossing the cervico-thoracic junction) to second distal vertebrae, as well as change in this angle by $<-10 \circ$ from the baseline and/or by clinical diagnosis, within 1 year (early) and $>1$ year (late) of surgery.

Results: Statistical analysis included 64 patients (mean age: $63.2 \pm 11.8$ years) undergoing long cervical deformity correction (median levels: $5,46.9 \%$ crossing the cervico-thoracic junction). Compared to late DJK, patients with age $>55$ years (OR: $9.05,95 \% \mathrm{Cl}: 1.14-71.8, \mathrm{p}=0.03)$, hypertension (OR: 9.97, 95\% $\mathrm{Cl}: 1.39-71.2$, $\mathrm{p}=0.02$ ), smoking at the time of surgery (OR: $8.64,95 \% \mathrm{Cl}: 1.01-77.2, p=0.05)$, and pre-operative $\mathrm{C} 2-\mathrm{C} 7$ lordotic angle $<14^{\circ}$ (OR: $\left.33.2,95 \% \mathrm{Cl}: 2.08-530.4, \mathrm{p}=0.01\right)$ were significant independent predictors of early DJK following PCF. However, patients with cardiac disease (OR: 23.9, 95\%Cl: 1.11-517.5, $p=0.05$ ), thyroid disorders (OR: $95.4,95 \% \mathrm{Cl}: 2.06-440.8, \mathrm{p}=0.02)$ and smoking at the time of surgery $(\mathrm{OR}: 12.1,95 \% \mathrm{Cl}: 1.78-$ 187.8, $p=0.05$ ) were significant independent predictors of late DJK.

Conclusion: Our results suggest that a surgical strategy to minimize the DJK might include a preoperative surgical reconstruction planning, however, smoking cessation and evaluation by medical team to manage the co-morbidities are also very important to alleviate the risk of this complication. 
7:36 - 7:38 am

\section{BMI Is Correlated with Functional Disability and Depression Following Adult Spinal Deformity Surgery}

Jianning Shao BA; Arbaz Momin; Jonathan J. Rasouli MD; Jacob Enders BSE; Edward C. Benzel MD; Michael P. Steinmetz MD

Introduction: Patients with high BMI are at increased risk for perioperative complications following adult spinal deformity (ASD) surgery. The increasing incidence of obesity renders a comprehensive understanding of the impact of BMI on patient outcomes following ASD surgery critical.

Methods: We retrospectively reviewed all patients who underwent surgical correction for ASD at our institution during the past 20 years and collected data on patient demographics, and quality of life (QOL) scores including Modified Oswestery Low Back Pain Disability (ODI) and PHQ9. All analyses were controlled for surgeon variability. Multivariate analysis was performed to determine whether BMI was independently correlated with postoperative QOL.

Results: A total of 600 patients were included in the final analysis: 198 (35.6\%) males and $358(64.4 \%)$ females. The average age was $59.8 \pm 16.7$ years, and the median preoperative KPS was 70 and the mean preoperative ODI was 52 . On multivariate analyses, BMI was independently and significantly correlated with worse postoperative ODI $(p=0.026)$ and $B M I \geq 30$ was correlated with higher scores of PHQ9 $(p=0.003)$. BMI was not significantly correlated with development of postoperative complications, PJK development, hardware failure, LOS, or readmission within 30 days.

Conclusion: Higher BMI is associated with increased functional disability and depression following ASD surgery, but does not seem to be a significant predictive factor of postoperative complications. BMI optimization in the preoperative period may improve quality of life outcomes in ASD patients. 
7:38 - 7:40 am

\section{Frailty Is An important Predictor of Proximal Junction Kyphosis Development Following Adult Spinal Deformity Surgery}

Jianning Shao BA; Arbaz Momin; Jonathan J. Rasouli MD; Jacob Enders BSE; Edward C. Benzel MD; Michael P. Steinmetz MD

Introduction: Proximal junctional kyphosis (PJK) is a common radiological complication of adult spinal deformity (ASD) surgery that significantly undermines deformity correction. Despite the paramount effect PJK on patients, its etiology is not well elucidated.

Methods: We retrospectively reviewed all patients who underwent surgery for ASD at our institution during the past 20 years and collected data on patient demographics, frailty measures, including Clinical Frailty Index (CFI), Charlson Comorbidity Index, modified frailty index ( $\mathrm{mFI})$, and preoperative Karnofsky Performance Scale (KPS), quality of life scores, and PJK development. Multivariate analyses and logistic regression were performed to determine variables independently correlated with and predictive of PJK development.

Results: 600 patients were included in the analysis: 198 (35.6\%) males and $358(64.4 \%)$ females. The average age was $59.8 \pm 16.7$ years, and the mean $\mathrm{CFI}, \mathrm{CCl}$, and $\mathrm{mFI}$ were $4.19 \pm 0.96,2.92 \pm 1.98$, and 0.12 \pm 0.10 . Median preoperative KPS was 70 . On multivariate analyses, preoperative KPS $(p=0.007)$ and preoperative pelvic tilt $(p=0.021)$ were significantly associated with PJK development. Logistic regression identified $\mathrm{CCl}(p=0.001)$ and preoperative pelvic tilt angle $(p=0.024)$ as significant predictors of PJK development.

Conclusion: Preoperative functional status and comorbidity burden predicts development of PJK following ASD. Patient frailty is an important contributor to PJK development and should be considered in the preoperative period. 
7:40 - 7:42 am

Pelvic Parameters Directly Influence S2 Alar-Iliac (S2AI) Screw Trajectory

Joseph Laratta; Jamal Shillingford; Jeffrey L. Gum MD; Andrew Pugely; Avery L. Buchholz MD; Steven D. Glassman; Ronald A. Lehman MD; Lawrence Lenke

Introduction: Spinopelvic fixation utilizing S2AI screws provides robust fixation across the lumbosacral junction.Freehand placement of such screws relies heavily on the rich surrounding sacropelvic anatomy. To date, no study has examined the relationship between spinopelvic sagittal parameters and screw trajectory.

Methods: Seventy-eight consecutive patients with degenerative lumbar pathology between 2015-2017 were reviewed. Preoperative standing X-rays were assessed to measure pelvic parameters including sacral slope, pelvic tilt, and pelvic incidence. Using 3-dimensional CT reconstructions, an ideal S2AI trajectory was defined. Sacral slope, horizontal angle, sagittal angle, intra-screw distance and estimated screw lengths were recorded.

Results: The average sagittal angle measured in the sagittal plane was $27.3+4.1^{\circ}$. The average horizontal angle measured in the axial plane using the posterior superior iliac spine as a reference was $35.9+3.9^{\circ}$. Maximum screw length and intra-sacral screw length were $109.7+16.4^{\circ}$ and $33.6+6.4^{\circ}$ respectively. Pelvic tilt was found to have a moderate inverse correlation with sagittal screw trajectory ( $r=-$ 0.467, p-value=0.006). Pelvic incidence and sacral slope had weak correlations with sagittal screw angle. In the subgroup analysis, patients with high pelvic tilt $>20^{\circ}$ had a significantly lower sagittal screw trajectory compared to those with a normal pelvic tilt $\left(24.9+3.7^{\circ}\right.$ versus $29.8+2.8^{\circ}$, p-value $\left.=<0.001\right)$.

Conclusion: Higher pelvic tilt or increased pelvic retroversion correlates to lower and flatter sagittal S2AI screw trajectories. This causal relationship is vital when planning for freehand S2Al screw placement. 
7:42 - 7:44 am

\section{Comparing and Contrasting the Clinical Utility of Sagittal Alignment Classification Frameworks:Roussouly vs.SRS-Schwab}

Cole Bortz BA; Peter G. Passias MD; Lafage Renaud; Christopher P. Ames MD; Bassel G. Diebo MD; Eric Klineberg MD; Frank Schwab; Justin S. Smith MD, PhD; Virginie Lafage PhD; ISSG International Spine Study Group

Introduction: Its important to compare the utility of sagittal alignment classification frameworks as they relate to ASD patient outcomes.

Methods: ASD patients were grouped by "theoretical" Roussouly type(1: $\mathrm{Pl}<45^{\circ}, \mathrm{LL}$ apex below $\mathrm{L} 4 ; 2: \mathrm{PI}<45^{\circ}$, LL apex above L4-L5 space; 3: $45^{\circ} \leq \mathrm{PI}<60^{\circ} ; 4$ : $\mathrm{PI} \geq 60^{\circ}$ ) and "current" (1: SS $<35^{\circ}$, LL apex below L4; 2 : $\mathrm{PI}<35^{\circ}$, LL apex above L4-L5; 3: $35^{\circ} \leq \mathrm{PI}<45^{\circ} ; 4: \mathrm{PI} \geq 45^{\circ}$ ), as published. Outcomes of those that mismatched "theoretical" and "current" types at both pre- and 2-years postop(2Y) were compared to those of preop mismatched patients that matched at $2 Y$ (Matched). Subanalysis compared those who improved in Schwab modifiers to those who did not.

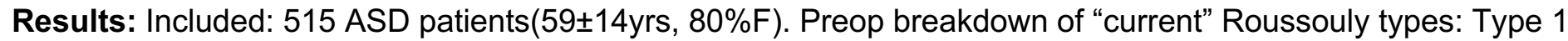
(10\%), 2 (54\%), $3(24 \%), 4(12 \%)$. Preop mismatch between "current" and "theoretical" types was $60 \%$. By $2 Y$, $16 \%$ of patients matched Roussouly types. Matched and Mismatched did not differ in rates of reaching MCID for any HRQL metrics by 2 Y(ODI, PCS, SRS Activity and Pain), nor rates of complications, reop, or PJK (all $\mathrm{p}>0.05)$, though Mismatched showed a trend of higher instrumentation failure(17\% vs $26 \%, p=0.08)$. By $2 Y$, $28 \%$ of patients improved in PT modifier, $37 \%$ in SVA, and $46 \%$ in PI-LL. Patients that improved in PT modifier reached MCID at higher rates for ODI and SRS Activity by $2 \mathrm{Y}$, and had lower rates of instrumentation failure than those that did not. Patients that both Matched Roussouly and improved in all Schwab modifiers by $2 Y$ met ODI and SRS-Activity MCID at higher rates than patients that did not. Match patients that improved in Schwab PT had less renal, infection, and neurologic complications; Match patients that improved in Schwab SVA had superior SF-36 and fewer cardiopulmonary complications at $2 Y(a l l p<0.05)$ than patients that did not.

Conclusion: Combined, both Roussouly and Schwab systems may offer utility in establishing targets for realignment. 
7:44 - 7:46 am

\section{Acute Failure Mechanisms of S2-Alar-lliac Screws for Pelvic Fixation in Adult Spine Deformity}

Kristen E. Jones MD; Christophe T. Martin MD; Jose San Miguel; Melissa Albersheim; Jonathan N. Sembrano MD; David W. Polly MD

Introduction: Pelvic fixation with S2-alar-iliac (S2AI) screws is a widely accepted technique in adult deformity surgery, with few descriptions of complications.

Methods: We performed a single-institution 20-month retrospective review of adult deformity cases $>4$ levels with bilateral S2AI screws, identifying cases with acute failure of pelvic instrumentation within 6 months postop. Exclusion criteria were age $<18$, nonambulatory status, or lack of 6-months postop xray.

Results: 7 of 107 cases had acute S2AI instrumentation failure (6.5\%). Failure cases averaged age 56.6 years, BMI 32.5, 57\% female, 12 levels of fusion, pre-op PI-LL mismatch 45 degrees, T1PA 38 degrees, and CSVL $6.5 \mathrm{~cm}$. Average deformity correction wasLLincrease 32 degrees, T1PA decrease 19 degrees, and CSVL decrease $3.5 \mathrm{~cm}$.

No screw breakage was seen. Failure mechanism was either set plug dislodgement from S2Al screw heads, sacral fracture/kyphosis with rod slippage out of S2Al set plugs, or both. 3 of $7(43 \%)$ failure patients had a nonmobile transitional segment at the lumbosacral junction and fractured through the transitional segment with kyphotic angulation. Salvage strategy was with two or more S2AI revision screws on each side.

Conclusion: 7 /107 patients had acute failure of S2AI instrumentation, via set plug disengagement or sacral/transitional segment vertebral fracture with rod breakage. All were high-magnitude deformity correction cases. Patients with large angular deformity correction and transitional lumbosacral anatomy may benefit from four total S2AI screws at the time of index surgery in order to prevent instrumentation failure. 
7:46 - 7:48 am

\section{Assessment of the Utility of Vertebral Body Hounsfield Units as a Predictor of Proximal Junctional Kyphosis}

Luke Wooster; Robert Winkelman BS; Dominic Pelle MD; Thomas Mroz; Michael P. Steinmetz MD

Introduction: Significant previous research has been done to characterize the utility of Hounsfield unit (HU) values of computed tomography (CT) attenuation as an alternative to dual energy x-ray absorption (DEXA) for the assessment of bone mineral density. Given that DEXA may be contraindicated in patients with severe scoliosis, assessment of the prognostic value of $\mathrm{HU}$ values in the adult spinal deformity population may improve pre-operative decision making.

Methods: HU measurements and radiographic measurements of kyphosis were made in 45 patients referred for surgical correction of adult spinal deformity. Radiographical sagittal alignment measures were made independently by two readers using scans acquired pre-operatively, at 6-weeks post-operation, and at latest follow-up. HU measures were made using three axial cuts of vertebral cancellous bone at the L1, L5, upper instrumented, and proximal vertebral levels using CT performed within one year of surgery. Proximal junctional kyphosis was defined as either an absolute proximal junctional angle of $>10$ degrees or a change from preoperation of $>10$ degrees.

Results: Overall prevalence of post-operative kyphosis was $44 \%$ in this cohort with a mean length of follow-up of 789 days. HU values at the L1, L5, upper instrumented, and proximal vertebral level were not significantly different between those patients who exhibited post-operative kyphosis and those who did not. The average $\mathrm{HU}$ values at the L1 and L5 vertebral levels were 117.9 and 111.8, respectively.

Conclusion: Pre-operative HU measures of vertebral bone density did not differ between those patients who went on to develop post-operative proximal junctional kyphosis and those who did not following correction of adult spinal deformity with lumbar to thoracic instrumentation. 
7:48 - 7:50 am

\section{Use of Intraoperative Rod Strain Sensors in Adult Spinal Deformity Surgery}

Andrew Ronald; Rolando M. Puno; Jeffrey L. Gum; Richard R. Navarro; Peter J. Baszuk; Leah Y. Carreon MD MSc; Douglas Orr; Michael P. Steinmetz MD

Introduction: Implanted hardware used for adult spinal deformity correction is placed under extremely high biomechanical forces as it holds the spine in a new, surgically induced, orientation. These forces, particularly rod strain, increase the risk of subsequent instrumentation failure. Intraoperative recording of rod strain may allow for recognition of instrumentation at risk for failure. Such recognition could allow for intraoperative adjustments of instrumentation orientation to reduce rod strain. We present a series of four patients who underwent surgical correction for adult lumbar spinal deformity with use of intraoperative rod strain sensors.

Methods: Four patients underwent surgical correction of lumbar spinal deformity with osteotomy and multilevel spinal fusion. Wireless rod strain sensors were used intraoperatively to record rod strain at different stages of implantation. Operating surgeons were blinded to the strain measurements. Follow up imaging was reviewed to assess status of implanted hardware. Laboratory recreation of intraoperative conditions was also used to assess how in-situ adjustment of rod orientation affects rod strain.

Results: Two patients showed intraoperative rod strain exceeding the rods yield during implantation. Only one patient showed rod strain exceeding yield at final orientation. One year follow up imaging in this patient showed hardware failure with a fractured pedicle screw. Laboratory recreation of the final hardware orientation in this patient showed that additional in-situ rod manipulation could have lowered the rod strain below its yield value.

Conclusion: Intraoperative monitoring of rod strain may allow surgeons to identify hardware constructs at risk for future failure and allow for intraoperative in-situ hardware manipulation to reduce this risk. Further research is needed to investigate the utility of intraoperative rod strain sensors in spinal deformity surgery. 
Spine Summit Meeting Abstracts, May 2021

7:50 - 7:52 am

\section{Depiction of Comorbidity Profiles in Adolescent Idiopathic Scoliosis Patients with and without Syringomyelia}

Katherine Pierce; Avery Brown; Cole Bortz BA; Haddy Alas; Sara Naessig; Jordan Manning; Bassel G. Diebo MD; Carl Paulino MD; Daniel M. Sciubba MD; Michael Gerling; Peter G. Passias MD

Introduction: The rarity of AIS-SM has resulted in a paucity of adequately powered studies investigating diagnoses unique to the concomitant diagnoses population.

Methods: The database was queried for ICD-9 codes pertaining to adolescent idiopathic scoliosis(737.1-3, 737.39, 737.8, 737.85, 756.1) patients aged 10-20yrs with concordant syringomyelia (AIS-SM) (336.0) from 2003-2012. Descriptive analyses assessed overall cohort demographics, comorbidities and diagnoses. Crosstabulations assessed frequencies of concomitant diagnoses.

Results: 77,183 AIS patients included(15.2yrs, $64 \% \mathrm{~F}) .0 .9 \%$ of the AIS patients had additional spine diagnoses including: herniated disc $(0.32 \%)$, spondylosis $(0.23 \%)$, disc degeneration $(0.21 \%)$ and stenosis $(0.16 \%)$. 821(1.2\%) of the AIS patients had concurrent SM, significantly greater incidence then nonAIS patients with $\operatorname{SM}(0.032 \%, p<0.001)$. Age of SM presentation: 10-13(40.5\%), 14-17(42.4\%) 18-20(17.1\%) years. AIS-SM patients were significantly younger(13.7 \pm 2.3 years, $p<0.001)$ with fewer females $(58 \%$, $\mathrm{p}<0.001)$. By organ system, AIS-SM patients presented with $74.1 \%$ pulmonary, $5.4 \%$ cardiovascular, $5.0 \%$ musculoskeletal, and $2.1 \%$ renal comorbidities. Pulmonary was the most frequent comorbidity between AIS $(45.3 \%)$ and AIS-SM(74.1\%),p<0.001. Amongst AIS patients, the following diagnoses had the strongest clusters with syringomyelia from greatest incidence to least: Arnold Chiari Malformation, Asthma, Tethered Cord, Hydrocephaly, Epilepsy, Hemiplegia, Quadriplegia, Pulmonary Failure, Malignancy, Restrictive Lung Disease, Spina Bifida. These top diagnoses were compared to the AIS-only population.

Conclusion: Within the isolated AIS cohort, concordant rates of syringomyelia amounted to $1.2 \%$ from $2003-$ 2012. Co-occurrences of other diagnoses included Arnold Chiari malformation, asthma, tethered cord, hydrocephaly, epilepsy, hemi- and quadriplegia, pulmonary failure. 


\title{
7:52 - 7:54 am
}

\section{The Effect of Hospital Characteristics on Pediatric Neuromuscular Scoliosis Fusion Cost}

\author{
Jonathan Dallas BS; Chevis Shannon; Christopher Bonfield
}

Introduction: Spinal fusion is used to treat pediatric neuromuscular scoliosis (NMS) by improving spine alignment and delaying disease progression. However, patients with NMS are often medically complex and require a higher level of care than other scoliosis patients, leading to increased treatment costs.

Methods: Patients were identified from the National Inpatient Sample (2012 Q1 2015 Q3). Inclusion criteria selected for patients with NMS, spinal fusion of at least four vertebral levels, and elective hospitalization. Sociodemographics, treating hospital characteristics, disease etiology/severity, comorbidities, length of stay, and hospital costs were collected. Univariable analysis and multivariable gamma log-link regression were used to determine hospital characteristics associated with changes in cost.

Results: 1780 weighted patients met inclusion criteria. Median cost was $\$ 68815$. Following multivariable regression, both small $(+\$ 11580, \mathrm{P}<0.001)$ and medium $(+\$ 6329, \mathrm{P}<0.001)$ hospitals had higher costs than large hospitals. Rural hospitals had higher costs than urban teaching hospitals $(+\$ 32438, P<0.001)$. Non-profit hospitals were more expensive than government $(-\$ 4518, P=0.030)$ and investor-owned $(-\$ 10240, P=0.001)$ hospitals. There was significant variability by US census division; as compared to the South Atlantic, all other divisions except for the Middle Atlantic had significantly higher costs, most notably the West North Central $(+\$ 15203, \mathrm{P}<0.001)$ and the Pacific $(+\$ 22235, \mathrm{P}<0.001)$. Hospital fusion volume was not associated with total cost.

Conclusion: A number of hospital factors were associated with changes in fusion cost. Larger hospitals may be able to achieve decreased costs due to economies of scale. Regional differences could reflect uncontrolledfor variability in underlying patient populations or systems-level and policy differences. Overall, this analysis identified multiple systemic patterns that could be targets of further cost-related studies and interventions. 
7:54 - 7:56 am

\section{Biomechanics of Pedicle Subtraction Osteotomy with Different Constructs: A Cadaveric Study of Stability and Strain.}

Bernardo De Andrada MD; Jakub Godzik; Jennifer Lehrman BSE, MS; Anna Newcomb; Juan Uribe; Brian Kelly; Jay D. Turner MD, PhD

Introduction: Pedicle subtraction osteotomy (PSO) is a powerful correction technique to restore lumbar lordosis but is associated with significant instability. Multi-rod configurations (XR) and TLIF or XLIF at the adjacent levels help attenuate the instability.

Methods: Standard pure moment tests $(7.5 \mathrm{Nm})$ followed by compression $(\mathrm{C})(400 \mathrm{~N})$ were performed on 14 specimens separated into two groups: 1)L3 PSO with L2-L3/L3-L4 TLIF (PSO+TLIF); 2)L3 PSO with L2-L3/L3L4 XLIF (PSO+XLIF). Each group had 3 different rod configurations investigated (Two-rods(2R), four-rod via auxiliary extra-rods $(\mathrm{aXR})$ and four-rods via deep-seated extra-rods(dXR). Conditions tested: 1)Intact; 2)Pedicle Screws and Rods(PSR); 3)PSO+2R; 4)PSO+aXR; 5)PSO+dXR. Posterior rods had strain gauges at the index level on the main and supplemental rods. Sacral Screw (SS) bending moments were recorded. Data were analyzed using RM-ANOVA or ANOVA ( $p<0.05)$.

Results: Within the TLIF group, aXR decreased ROM during flexion(FL) and extension(EX) $(p<0.016)$ compared to $2 R$. The $d X R$ decreased ROM during EX ( $p=0.009)$. Within the XLIF group, both aXR and $d X R$ decreased motion compared to $2 R$ during axial rotation(AR) $(p<0.031)$. In XLIF group, dXR had increased $S S$ strain compared to aXR in Compression $(C)$ and right Lateral Bending (LB) $(p<0.033)$. During FL and EX, PSO+TLIF+2R had increased RS compared to PSO+XLIF+2R $(p<0.039)$. During FL, EX and LB, in XLIF group, $d X R$ increased $R S$ compared to aXR, in TLIF group this only appeared in $E X(p<0.027)$. In XLIF group, aXR increased XR RS compared to dXR in FL $(p<0.001)$ and LB $(p<0.018)$.

Conclusion: The protective effect of XR on RS seems more effective in TLIF than XLIF. Deep-seated configuration induces strain at the main rod and at the sacral screw. Constructs with aXR using connectors has ability to share strain between main and supplemental rods. 
7:56 - 7:58 am

\section{Changes in Spinopelvic Parameters in the first three months following Corrective Surgery for Adult Spinal Deformity}

Ahmed Albayar; Ashwin G. Ramayya MD, PhD; Abigail Roche; Patricia L. Z. Sullivan MD; Jang Yoon; Comron Saifi; Zarina S. Ali MD; William C. Welch MD, FACS, FICS; Vincent Arlet MD; Ali K. Ozturk MD

Introduction: Little is known about changes in spinal alignment parameters in the first few months following corrective surgery for adult spinal deformity (ASD). Typically, surgeons follow patients with serial radiographs, however patient posture and overall alignment can vary with postoperative pain, bracing and other external variables.

Methods: Single-Institution records of ASD patients who underwent deformity surgeries from 2013 to 2018 were reviewed. Patients $<40$ years old, had $<6$ levels fused, had acute proximal junctional kyphosis or failure, or lacked 3-month postoperative radiographs were excluded. Lumbar lordosis (LL), thoracic kyphosis (TK) and sagittal vertical axis (SVA) values at immediate and 3-month postoperative time-points were measured using Surgimap ${ }^{\circledR}$ spine software, then statistically compared via two-sample student t-test. Furthermore, TK differences in patients with upper thoracic to pelvis (UT-P) fusions vs lower thoracic to pelvis (LT-P) fusions were compared via paired t-test.

Results: Thirty-six patients (24 females, 67\%) with a mean age of 61.5 (range, $40-75$ years) years were included. Spine alignment comparisons showed a statistically significant increase in TK at the 3-month postoperative time-point $(p=0.006)$. In addition, wide variations in SVA (variation range, $47--144 \mathrm{~mm}$ ) were noted, however, this did not reach statistical significance, likely due to the changes being in both positive and negative directions ( $p=0.21, p=0.18$, respectively). TK changes at 3-months postoperatively did not significantly vary when compared in UT-P vs LT-P groups.

Conclusion: Our results suggest that as post-operative pain subsides and the body settles into its new alignment, significant changes occur in spinopelvic parameters in the subacute period following surgery. Clinicians may benefit from imaging at a 3-month postoperative timepoint. 
7:58 - 8:00 am

\section{Incidence and Risk Factors of Mechanical Complications After Osteotomies for Correction of Cervicothoracic Deformities}

Darryl Lau; Rushikesh S. Joshi BS; Alexander F. Haddad BS; Vedat Deviren MD; Christopher P. Ames MD

Introduction: Inflexible cervicothoracic deformities often require multilevel or high-grade osteotomies in order to achieve adequate correction. The rate and risk factors of hardware failure is not well defined.

Methods: A retrospective review of consecutive patients that underwent cervicothoracic deformity correction from 2010 to 2018 was performed. Inclusion criteria was cervical kyphosis $>20$ degrees and/or cSVA $>4 \mathrm{~cm}$. Mechanical complications were evaluated at 1 and 2-year follow-up. Multivariate analysis was used.

Results: 83 patients had 1-year follow-up, and 51 of 73 (69.9\%) had 2-year follow-up. Mean age was 63.4 years, and $61.0 \%$ were female. $53.0 \%$ of patients underwent $3 \mathrm{CO}$. Preoperative and postoperative measures significantly improved: cSVA (6.2 vs. $4.6 \mathrm{~cm}, p<0.001)$, cervical lordosis $(6.3 \mathrm{vs} .-8.3 \mathrm{~cm}, \mathrm{p}<0.001)$, cervical scoliosis (6.5 vs.2.2 degrees, $p<0.001$ ), and T1-slope (41.7 vs. 36.3 degrees, $p=0.007)$. Mechanical complication rate at 1-year was $28.9 \%$ : junctional failure $(18.1 \%)$, implant failure $(16.9 \%)$, and pseudarthrosis (10.8\%). At 2-years, mechanical complication rate was $35.3 \%$ : junctional failure $(21.6 \%)$, implant failure (21.6\%), and pseudarthrosis (15.7\%). Reoperation rate at 1-year and 2-years were $24.1 \%$ and $27.5 \%$. Higher $\mathrm{BMI}$ and presence of preoperative cervical scoliosis were independently associated with higher odds of mechanical failure $(p=0.020$ and $p=0.014)$ and reoperation $(p=0.043$ and $p=0.014)$.

Conclusion: Mechanical complications are high at 1- and 2-years following cervicothoracic deformity correction. High BMI and preoperative scoliosis predispose patients for hardware failure. Large multicenter prospective studies are needed. 
8:00 - 8:02 am

\section{Significance of Posterior Fusion Bone Density on Mechanical Complications Following Thoracolumbar Three-column Osteotomy}

Darryl Lau; Jae Hwan Cho; Vedat Deviren MD; Christopher P. Ames MD

Introduction: Few studies have evaluated the relationship of fusion mass bone density to mechanical complications following thoracolumbar three column osteotomy $(3 \mathrm{CO})$.

Methods: A retrospective review of a consecutive cohort of ASD patients who underwent thoracolumbar 3CO from 2007 to 2017 was performed. All patients had 1-year CT and at least 2-year follow-up. Posterior fusion mass bone density was evaluated by measuring Hounsfield Unit (HU) on CT at the UIV, LIV, and osteotomy site. Fusion mass bone density was compared in patients with and without mechanical complications with multivariate analysis.

Results: 165 patients (63.2 years, 33.5\% male) were included. Overall PJK rate was 18.8\%, and 6.7\% underwent PJK revision. Overall RF rate was $34.5 \%$ and $20.6 \%$ underwent revision for RF.Among 57 patients with RF, 71.9\% had pseudarthrosis. Sagittal parameters improved significantly: SVA (111.8 to $51.6 \mathrm{~mm}$, $\mathrm{P}<0.001$ ), and PI-LL (36.1 to 11.1 degrees, $\mathrm{P}<0.001)$. Fusion mass density did not differ between patients with or without RF. However, in RF patients with pseudarthrosis, there was significantly higher fusion mass density near the osteotomy compared to those without pseudarthrosis $(515.7 \mathrm{HU}$ vs. $354.2 \mathrm{HU}, \mathrm{P}=0.012)$. In regards to PJK, there was significantly lower density of posterior fusion mass at the UIV in patients who experienced PJK compared to patients without PJK (431.5HU vs. 537.4HU, $\mathrm{P}=0.035)$.

Conclusion: Fusion mass density on CT is not correlated to RF, but greater bone density near the osteotomy was correlated to accompanying pseudarthrosis in patients with RFs. Patients with PJK tend to have less dense posterior fusion mass at the UIV. Assessing density of fusion mass on CT may be helpful in assessing the risk for RF in patients with pseudarthrosis and PJK. 
8:02 - 8:04 am

\section{Anterior Column Realignment: A Single Institution Retrospective Series}

Meng Huang MD; Saeed S. Sadrameli MD, MS; Vitalily Davidov; Jonathan J. Lee; Suraj Sulhan; Virendra Desai; Sean Barber; Blake Staub MD; Todd W. Trask; Paul J. Holman

Introduction: Severe sagittal deformities traditionally require highly morbid three column osteotomies for correction $^{1,3,5}$. MIS techniques for deformity correction have gained popularity including the lateral trans-psoas anterior column realignment (LLIF-ACR), which leverages hyperlordotic (HL) interbody grafting and anterior longitudinal ligament release $\mathrm{e}^{2,4,6}$. The safety and efficacy compared to PSO is not well established.

Methods: We performed a retrospective chart review of 50 degenerative scoliosis and iatrogenic flat back patients who underwent 62 levels of LLIF-ACR as part of a hybrid MIS/open thoracolumbar construct. Sagittal parameters, ACR segmental lordosis, intra- and post-operative complications, and long term outcomes were reviewed.

Results: Thirty-two and thirty levels of $30^{\circ}$ and $20^{\circ} \mathrm{HL}$ implants and were placed, respectively. Forty-nine levels also received a supplemental posterior Ponte osteotomy. The average increase in segmental lordosis was $17.09^{\circ} \pm 10.82$ and $13.2^{\circ} \pm 8.78$, respectively for $30^{\circ}$ and $20^{\circ}$ implants. There was only one complication directly attributable to the ACR. This was an unrecognized injury to the segmental artery by the graft retention screw. This required endovascular embolization due to expanding retroperitoneal hematoma post-operatively. One temporary femoral nerve neuropraxia occurred during a separate non-ACR level. There was one pseudomeningocele, non-ACR lateral cage extrusion, sacral fracture, and two posterior wound infections that required re-operation. Nineteen medical complications were noted, and only one was serious. A patient developed C. diff colitis and severe sepsis and expired on the same admission. Average EBL for the ACR stage, which included all other lateral and ALIF levels, was $329 \mathrm{cc}$. All global sagittal parameters were adequately corrected. Median follow up was 12.5 months, and average ODI improved from $52.88 \pm 2.31$ to $30.44 \pm 3.59$. Solid arthrodesis was either plain film or CT $(77 \%)$ confirmed in all patients.

Conclusion: LLIF-ACR is an effective MIS alternative for achieving powerful sagittal correction without unacceptable morbidity. 
8:04 - 8:06 am

\section{Frailty Does Not Negatively Affect Cost Utility in Adult Spinal Deformity}

Avery Brown; Katherine Pierce; Cole Bortz BA; Sara Naessig; Haddy Alas; Waleed Ahmad; Aaron J. Buckland MBBS, FRACS; Themistocles Protopsaltis MD; Shaleen Vira; Peter G. Passias MD

Introduction: Frailty Does Not Negatively Affect Cost Utility in Adult Spinal Deformity

Methods: Included: surgical ASD (scoliosis $\geq 20^{\circ}, \mathrm{SVA} \geq 5 \mathrm{~cm}, \mathrm{PT} \geq 25^{\circ}$, or thoracic kyphosis $\geq 60^{\circ}$ ) patients $\geq 18$ years with available frailty and ODI data at baseline (BL) and 2-years post-surgery (2Y). Independent T-Tests assessed BL radiographic differences in PT, PI-LL, and Schwab SVA modifier status between NF vs. F/SF groups. Utility data was calculated using the ODI converted to the SF-6D. QALYs utilized a 3\% discount rate to account for decline to life expectancy (LE). Costs were calculated using the PearlDiver database. After accounting for complications, LOS, revisions, and death, cost per QALY at 2Y and LE were calculated for NF, and F/SF patients. ICER was compared between non-operative (non-op.) and operative (op.) NF and F/SF patients at $2 Y$ and LE.

Results: Included: 79 ASD patients. Descriptive statistics for the cohort were: age $51.0 \pm 6.8,76 \% \mathrm{~F}$, BMI 26.7 $\pm 6.8,54 \%$ osteotomy, $54 \%$ decompression, and $11.6 \pm 4.2$ mean levels fused. There were no differences in PT, PI-LL, or severe SVA Schwab modifier grades between NF or F/SF (all $p>0.05$ ). When compared to nonop. ASD, the ICER was $\$ 447,943.96$ vs. $\$ 313,211.01$ for NF and F/SF at 2 Y, and $\$ 68,311.35$ vs. $\$ 47,764.61$ for NF and F/SF at LE.

Conclusion: F and SF patients had lower cost per QALY compared to NF at 3Yand LE. In addition, when compared to a non-op. cohort of ASD, F and SF patients had lower ICER values. While these results support op. correction of F and SF groups, it is notable that they start at a more disabled BL with more opportunity to improve. Additionally, there may be a threshold of frailty that is non-op. due to a risk of complications not captured here. Future research should investigate economic outcomes at extended follow-ups, but these results support the cost effectiveness of ASD surgery at all states. 
8:06 - 8:08 am

\section{A Systematic Review of Operative and Non-operative Management for Scheuermann Kyphosis}

Sakibul Huq; Jeffrey S. Ehresman BS; Ethan Cottrill BS; A. Karim Ahmed; Zach Pennington BS; Erick M Westbroek MD; Daniel M. Sciubba MD

Introduction: The optimal management of Scheuermann Kyphosis (SK) remains a subject of substantial debate. Nonoperative management classically employs bracing, while operative management involves either a combined anterior release and fusion plus posterior fusion or a posterior-only fusion.

Methods: Following PRISMA guidelines, we systematically reviewed the English literature between 1950 and 2017 for all full-text publications on SK management.

Results: 45 studies covering 1829 patients were included in our analysis. The most commonly reported indications for intervention were deformity, pain, failing non-operative treatment, and neural impairment. Posterior-only surgery afforded superior correction compared to combined anterior-posterior surgery. There was no difference in loss of correction between surgical approaches. Both surgical approaches provided greater correction and less loss of correction than bracing. The most commonly reported complications of surgery were instrumentation failure and junctional kyphosis. Anterior-posterior surgery had a wider complication profile than posterior-only surgery. Surgeon preferences have largely shifted from combined anterior-posterior to posterior-only approaches over the last two decades.

Conclusion: For SK patients, surgery provides greater correction and maintenance of correction than bracing. Posterior-only surgery may provide superior correction, similar loss of correction, and a narrower complication profile compared to combined anterior-posterior surgery. These findings may explain the increased popularity of posterior-only surgery in recent years. 
8:08 - 8:10 am

\section{Intraoperative Long Cassette Films and Pelvic Kickstand in Long Thoracolumbar Adult Deformity Correction}

Ryan C. Hofler MD, MS; Russ P. Nockels MD

Introduction: Maintenance of normal coronal balance can be a challenge in select cases of adult spinal deformity correction.

Methods: Patients with adult thoracolumbar spinal deformity between October 2017 and June 2019 with coronal plane deformity undergoing thoracolumbar fixation and deformity correction had intraoperative anteroposterior long cassette radiographs (APLCR) from fusion apex to sacrum prior to final rod placement. Coronal deviation of the apex of the construct was measured as the horizontal distance between the apex level plumb line to the central sacral vertical line (APL-CSVL). In patients with persistent intraoperative coronal deviations, a Kickstand (a supplemental rod anchored to the ilium ipsilateral to the deviation) was used to apply a lateralizing force to reduce the degree of deviation. APL-CSVL was measured on APLCR pre-, intra-, and postoperatively.

Results: A total of 15 patients underwent T3-Ilium fusion with spinal deformity correction with intraoperative APLCR; 7 underwent Kickstand placement. Mean preoperative APL-CSVL was similar between cohort $(4.3 \mathrm{~cm}$ vs $2.2 \mathrm{~cm}, p=0.09)$, while intraoperative APL-CSVL was greater in the kickstand cohort $(4.3 \mathrm{~cm}$ vs $0.6 \mathrm{~cm}, 9<$ $0.001)$. Mean postoperative APL-CSVL was similar between groups $(2.1 \mathrm{~cm}$ vs $1.8 \mathrm{~cm}, p=0.37)$. Patients with kickstands demonstrated significant reduction in APL-CSVL postoperatively $(p=0.01)$.

Conclusion: Intraoperative APLCR during long segment thoracolumbar deformity correction provided excellent screening for persistent coronal imbalance. Kickstand applications allowed for excellent maintenance of coronal balance. 
8:10 - 8:12 am

\section{Predictors of Reoperation in Medicare Patients Undergoing Primary Adult Thoracolumbar Deformity Surgery}

Kunal Varshneya; Parastou Fatemi MD; Zachary A. Medress MD; John K. Ratliff MD, FACS; Anand Veeravagu MD

Introduction: Previous literature reports estimates $20 \%$ of patients undergoing thoracolumbar adult spinal deformity (ASD) surgery require another surgery within 2 years. Most of the published data discusses the risk factors for revision surgery in the general population, and little data exists describing these in a Medicare cohort.

Methods: We queried the MarketScan Medicare supplemental database in order to identify patients who were diagnosed with a spinal deformity and underwent ASD surgery from 2007-2015. Patient level factors and revision risk were investigated during two years after primary ASD surgery. Patients without Medicare insurance or those with any prior history of trauma or tumor were excluded from this study.

Results: A total 2564 patients enrolled in Medicare underwent ASD surgery during $2007-2015$ in the dataset. Median age at diagnosis was 71.5 years, and a majority of patients (68.5\%) were female. At 2-years follow-up, $25.8 \%$ of patients required a reoperation. Osteoporosis (OR 1.48, p < 0.05), CHF (OR 1.34, p < 0.05) and paraplegia (OR 2.41, $p<0.05$ ) independent increased odds for revision surgery. The use of intraoperative BMP was protective against reoperation (OR 0.71, $\mathrm{p}<0.05$ ). Among 90-day complications, wound complications were the strongest predictor of revision surgery (OR 2.85, $p<0.05$ ). The development of a pulmonary embolism (PE) also increased the odds of revision surgery by $1.84 x(p<0.05)$.

Conclusion: Approximately 1/4 Medicare patients failed primary ASD surgery within 2 years. Baseline comorbidities such as osteoporosis and CHF and short-term complications such as PE and wound complications significantly increased the odds of operative failure. 
8:12 - 8:14 am

\section{Single-Position Lateral Surgery with Transpsoas Fixation is an Effective Treatment for Adult Spinal Deformity}

Joseph Laratta; Jeffrey L. Gum MD; Avery L. Buchholz MD; Andrew Pugely; William Smith

Introduction: The minimally-invasive lateral approach for lumbar interbody fusion (LLIF) is being increasingly indicated for adult scoliosis and sagittal deformities. Single-position techniques allow concurrent access to anterior and posterior columns. Traditionalfixationis obtained with pedicle screws, however,transpsoassegmental screw-rod constructs are a novel option.

Methods: Forty-five consecutive adult degenerative scoliosis patients with significant sagittal malalignment $\left(\mathrm{PI}-\mathrm{LL} \geq 20^{\circ}\right.$ ) treated with single-position, multi-level LLIF and lateral rod fixation were included in the analysis. All patients underwent postoperative CT scan at 24 months to evaluate fusion and iliopsoas volume. Patientreported outcomes were assessed by visual analog scale (VAS) and Oswestry Disability Index (ODI).

Results: At final follow-up, the PI-LL mismatch improved from $29.1^{\circ} \pm 19.1^{\circ}$ to $11.1^{\circ} \pm 6.4^{\circ}(p<0.0001)$. The coronal Cobb angle improved from $28.1^{\circ} \pm 8.9^{\circ}$ to $6.1^{\circ} \pm 7.3^{\circ}(p<0.0001)$. VAS for low back pain declined from 8.4 preoperatively to 3.5 at final follow-up, with $67 \%$ of patients reporting more than a 4-point improvement in VAS score. ODI decreased from 61 to 28 at two-year follow-up. Total psoas area was unchanged at 2-years postoperatively $(p=0.061)$. Additionally, there was no evidence of pseudarthrosis or proximal junctional kyphosis in the cohort at final follow up.

Conclusion: Single-position surgery with multi-level LLIF and lateral segmental screw-rod fixation is safe and effective in the treatment of ASD. 
8:14 - 8:16 am

\section{One vs Two Attending Surgeons for Spine Deformity: A Systematic Review and Meta-analysis}

Asad M. Lak MD; Abdullah M. Abunimer MD; Caroline Goedmakers; Rania Mekary; Linda Aglio; Melvin Makhni; Hasan A. Zaidi BS, MD

Introduction: Surgical correction of spine deformity is associated with high rate of complications. Various approaches have been utilized to reduce the rate of complications.

Methods: MEDLINE, Embase, Web of science and Cochrane databases were last searched on September 03, 2019. A total of 750 records were identified excluding duplicates. After screening, 9 studies were included.

Results: A total of 915 patients were analyzed. The mean age was 33.0 years in the single surgeon and 33.3 years in the dual surgeon group. Compared to a dual surgeon approach, the mean number of levels fused (mean difference $=0.23 ; 95 \% \mathrm{Cl}:-0.58,1.03$ ) as well as blood loss, though higher in the single-surgeon group, were non-significant (mean difference $=179.3 \mathrm{~mL} ; 95 \% \mathrm{Cl}:-27.3,386)$. Operative time (mean difference = $82.7 \mathrm{~min} ; 95 \% \mathrm{Cl}: 49.9,115.4$ ) and length of hospital stay (mean difference $=0.85$ days; $95 \% \mathrm{Cl}: 0.58,1.12$ ) were however, significantly higher in the single-surgeon group. Regarding complications, there were no differences in terms of neurological (Risk Ratio $=1.56$; $95 \% \mathrm{Cl}$ : $0.62,3.90$ ), instrumental (Risk Ratio $=1.15$; $95 \% \mathrm{Cl}: 0.19,6.81$ ) or infectious (Risk Ratio $=2.19 ; 95 \% \mathrm{Cl}: 0.99,4.87$ ) complications.

Conclusion: Dual surgeon approach is associated with reduced operative time and length of hospital stay and can potentially improve surgical outcomes for spine deformity as compared to a single surgeon approach. 


\section{8:16 - 8:18 am}

\section{Influence of Gender on Discharge Disposition After Spinal Fusion for Adult Spine Deformity Correction}

Aladine A. Elsamadicy BE; Isaac G. Freedman BPhil, MPH; Andrew B. Koo; Wyatt David; Megan Lee; Amber King; Adam Kundishora MD; Gregory A. Kuzmik MD; Ramana Gorrepati MD; Christopher S. Hong MD; Joaquin Camara-Quintana; Luis Kolb MD; Maxwell Laurans; Khalid M. Abbed MD

Introduction: The influence of gender disparities on discharge disposition after adult spine deformity correction (ASD) is relatively understudied.

Methods: The Nationwide Inpatient Sample database (2011-2014) was queried for patients with ASD ( $\geq 26$ years-old) and elective spine fusion surgery involving $\geq 4$ levels using ICD-9 codes. Patients were stratified by gender: Male or Female. Multivariate linear and logistic regressions were used to assess the impact of gender on length of hospital stay and discharge disposition.

Results: A total of 4,972 patients were identified of which $3282(66.0 \%)$ were Female and $1690(34.0 \%)$ were Male. The Male cohort had a higher prevalence of comorbidities than the Female cohort. There was a difference in the number of levels operated on between cohorts, with the Female cohort having fewer 4-8-level fusionsand more 9+-level fusionscompared to Males. The Female cohort had greater rates of postoperative UTland surgical site hematomas, while the Male cohort had more postoperative MI. The Female cohort spent slightly more time in the hospital than Male cohort. Female patients had a significantly greater proportion of non-routine discharge dispositioncompared to Male patients. However, in a multivariate analysis including patient and hospital factors, gender was not an independent predictor of discharge disposition, but was independently associated with increased LOS.

Conclusion: Our study suggests gender disparities may not have a significant impact on discharge disposition after spinal fusion for ASD involving 4 levels or greater. 
8:18 - 8:20 am

\section{Management of the Fractional Curve in Degenerative Scoliosis using Circumferential Minimally Invasive Fusion}

Corey T. Walker MD; Jakub Godzik MD, MSc; Jay D. Turner MD, PhD; Juan S. Uribe MD, FAANS

Introduction: Management of the fractional curve is an important part of the treatment of degenerative scoliosis, particularly in patients with Type C coronal malalignment (shift towards the major curve convexity). Correction of the fractional curve remains a challenge for minimally invasive deformity correction.

Methods: A single-institution retrospective radiographic evaluation of patients with degenerative scoliosis $\left(\mathrm{Cobb}>20^{\circ}\right)$ treated with cMIS approaches (lateral transpsoas fusion (LLIF) combined in some cases with anterior lumbar interbody fusion (ALIF) and/or percutaneous pedicle screw fixation) was performed. Coronal fractional curves were assessed using L4 and L5 coronal tilt angles based on standing full-length scoliosis radiographs pre-operatively and at final follow-up.

Results: 40 patients were treated with MIS deformity approaches. Of those patients, 19 had an L4/5 LLIF and ALIF at L5/S1, which resulted in an average improvement of $4.8^{\circ}$ in the L5 tilt and $8.5^{\circ}$ in the L4 tilt. 16 patients had an LLIF at L4/5 without an ALIF at L5/S1, which resulted in substantially less improvement of $0.3^{\circ}$ in the $\mathrm{L} 5$ tilt and $5.7^{\circ}$ in the $\mathrm{L} 4$ tilt ( $p=0.03,0.001$, respectively). Of the 11 patients with a Type $C$ coronal malalignment pre-operatively, the 6 patients that had correction of their alignment had an improvement in their L4 tilt of $9.3^{\circ}$ compared to $3.6^{\circ}$ in the 5 patients who remained with malalignment post-operatively $(p<0.05)$. This occurred while having similar improvements in primary coronal curve cobb angles $(p>0.05)$.

Conclusion: While L4/5 LLIF improves the coronal fractional curve in degenerative scoliosis, the addition of an L5/S1 ALIF results in even greater correction. Treatment of Type C curves remains difficult for MIS deformity surgeons, and is reliant on appropriate treatment of the fractional curve using a combination of L4/5 LLIF and L5/S1 ALIF. 
8:20 - 8:22 am

\section{Frailty Predicts Functional Outcome Following Adult Spinal Deformity Surgery}

Jianning Shao BA; Arbaz Momin; Jonathan J. Rasouli MD; Jacob Enders BSE; Edward C. Benzel MD; Michael P. Steinmetz MD

Introduction: Adult spinal deformity (ASD) is associated with high complication rates and requires meticulous patient selection to optimize outcomes. Quality of life (QOL) following ASD surgery has emerged as a particularly important question, especially in the frail population.

Methods: We retrospectively reviewed all patients who underwent surgical correction for ASD at our institution during the past 20 years and collected data on patient demographics, frailty measures, including Clinical Frailty Index (CFI), Charlson Comorbidity Index, modified frailty index ( $\mathrm{mFI})$, preoperative Karnofsky Performance Scale (KPS), and quality of life scores. Multivariate analyses and logistic regression were performed to determine variables independently correlated with QOL and predictors of poor postoperative QOL.

Results: 600 patients were included in the analysis: 198 (35.6\%) males and $358(64.4 \%)$ females. The average age was $59.8 \pm 16.7$ years, and the mean $\mathrm{CFI}, \mathrm{CCl}$, and $\mathrm{mFI}$, were $4.19 \pm 0.96,2.92 \pm 1.98$, and 0.12 \pm 0.10 . Median preoperative KPS was 70 . On multivariate analyses, CFI $(p<0.001)$ and preoperative KPS $(p<0.001)$ were significantly associated with functional outcomes. Logistic regression analysis identified CFI and preoperative KPS as significant predictors of poor postoperative functional outcomes, defined as KPS scores below 80. Age was not significantly correlated with or predictive of postoperative functional outcomes.

Conclusion: CFI and preoperative functional status are important predictors of QOL following ASD surgery. Age does not accurately inform postoperative QOL, and should be considered in the context of a comprehensive frailty evaluation. 
Spine Summit Meeting Abstracts, May 2021

8:22 - 8:24 am

\section{Preoperative Pelvic Tilt Informs Functional Outcomes After Adult Spinal Deformity Surgery in the Lumbar Spine}

Jianning Shao BA; Arbaz Momin; Jonathan J. Rasouli MD; Jacob Enders BSE; Edward C. Benzel MD; Michael P. Steinmetz MD

Introduction: Adult spine deformity (ASD) surgery plays a critical role in the restoration of sagittal balance and achievement of spinopelvic harmony in ASD patients. There is relatively little known about the relationship between preoperative sagittal alignment parameters and functional outcomes following ASD surgery.

Methods: We retrospectively reviewed all patients who underwent surgical correction for ASD at our institution during the past 20 years and collected data on patient demographics, pelvic tilt (PT), and functional scores including Modified Oswestery Low Back Pain Disability (ODI) and Karnofsky Performance Scale (KPS) scores. All analyses were controlled for surgeon variability. Multivariate analysis and logistic regression was performed to identify factors independently correlated with and predictive of postoperative functional status. Cut-points were values that maximized the area under the receiver operating curve.

Results: A total of 600 patients were included in the final analysis: 198 (35.6\%) males and 358 (64.4\%) females. The average age was $59.8 \pm 16.7$ years, and the median preoperative KPS was 70 and the mean preoperative ODI was 52 . On multivariate analyses, preoperative PT $(p=0.002)$ was significantly correlated with postoperative KPS scores. Logistic regression also found preoperative PT $(p=0.002)$ predictive of postoperative KPS, with a statistically significant cut-point of $39.9^{\circ}(p=0.006)$.

Conclusion: Preoperative PT is an important predictor of functional outcome following ASD surgery of the lumbar spine. Pelvic tilt angles greater than 39.9 is significantly correlated with poor postoperative functional outcomes following ASD surgery. 
8:24 - 8:26 am

\title{
Circumferential Correction of Spinal Deformity and Instability Secondary to Bacterial Spondylodiscitis
}

\author{
Ziev Moses MD; Mazda K .Turel MD; Mena G. Kerolus MD; Samuel Meza; Ricardo B. V. Fontes MD, PhD
}

Introduction: Spinal deformity following spondylodiscitis is a variably reported clinical entity with several suggested treatment strategies. The presence of advanced deformity makes non-operative care impractical due to extensive destruction of the anterior column.

Methods: Retrospective chart review of 18 patients who underwent a three-column osteotomy for deformity due to spondylodiscitis during a 48-month period. Pre-operative data included co-morbidities, ambulatory status, infectious history, and VAS scores. Peri-operative variables are reported. Outcome variables included VAS scores, ambulatory status, ability to care for self, and radiographic deformity correction.

Results: Mean age (8 male; 10 female) was 58.8 years. All patients except one underwent a posterior threecolumn osteotomy in 1 or 2 stages. The average number of levels fused was 9 , average duration of surgery was 8.3 hours and mean intraoperative estimated blood loss was $2900 \mathrm{~mL}$. Mean preoperative VAS was $8.6 \pm$ 2.2 and all patients were bedridden, improving to $2.4 \pm 2.9$ postoperatively and all patients except four regained ambulatory status and complete self-care, at a mean follow-up period of 14.8 months. One patient died in the perioperative setting from pulmonary embolism and there were 5 reoperations in the study period. Mean preoperative segmental kyphosis of $26 \pm 12$ degrees was corrected to $8 \pm 19$ degrees.

Conclusion: This study suggests extensive circumferential reconstruction for deformity secondary to spondylodiscitis can be effective in restoring these sick patients to self-care and ambulatory status. Reconstruction is feasible and may be considered even during the subacute phase of infection. 
8:26 - 8:28 am

\section{Intravenous Tranexamic Acid and Perioperative Blood Loss in Percutaneous Three-Level Lumbar Fusion: A Feasibility Study}

Robert W. McCabe DO; Doris Tong; Lucas Garmo; Teck Mun Soo

Introduction: Tranexamic Acid (TXA) is effective at reducing perioperative blood loss in open lumbar fusion but its effectiveness in MIS lumbar fusion is uncertain. We sought to demonstrate that intravenous (IV) TXA would lead to a decrease in perioperative blood loss and transfusion necessity in elective 3-level MIS transforaminal interbody lumbar fusion (TLIF).

Methods: We retrospectively reviewed consecutive patients' charts who underwent elective 3-level MIS-TLIF from 8/2017-6/2019. Perioperative TXA use was defined as one gram or $10 \mathrm{mg} / \mathrm{kg}$ of TXA administered intravenously immediately prior to incision. Perioperative blood loss was defined as hemoglobin reduction from preoperative baseline to the lowest postoperative level. Comparability between groups was evaluated for age, sex, BMI, smoking, osteoporosis, and preoperative anticoagulant use. Feasibility was evaluated by the perioperative blood loss in the control group. Safety was evaluated by comparing the incidence of venous thromboembolism (VTE) among the groups. Efficacy was evaluated by the difference between perioperative blood loss and number of blood units transfused between the groups. Secondary outcomes were length of stay and operative time. Univariate analyses were used. Statistical significance was considered at $p \leq 0.05$.

Results: We studied 30 patients. Seven patients received TXA. Feasibility was demonstrated by clinically significant hemoglobin reduction in the controls $(4.5 \pm 1.5 \mathrm{~g} / \mathrm{dL})$. Safety was demonstrated by the incidence of VTE in TXA vs. control ( 0 vs. 8.7\%, $p=0.42$ ). Efficacy was demonstrated by significantly different postoperative hemoglobin reduction (2.94 TXA vs $4.50 \mathrm{~g} / \mathrm{dL}$ control, $\mathrm{p}=0.01)$. Number of units transfused, operative time, and length of stay were not significantly different between the groups.

Conclusion: We demonstrated that perioperative blood loss was significant in the controls for 3-level or greater MIS-TLIF and TXA was efficacious in significantly reducing this loss without an increase in VTE. Thus, a randomized controlled trial would be feasible. 


\section{8:28 - 8:30 am}

\section{Patient Expectation on Outcomes of Adult Spinal Deformity Surgery}

W. H. Andrew Ryu BSc, MSc, MTM, MD; Brian T. David PhD; Ricardo B. V. Fontes MD, PhD; Richard G. Fessler MD, PhD

Introduction: There has been a growing interest in examining patient expectation in the setting of spine surgery, particularly as a potential predictor of postoperative satisfaction. However, patient expectation has never been studied in the setting of adult spinal deformity (ASD).

Methods: Retrospective chart review was performed on patients who underwent deformity correction surgery between 2016-2018 involving at least 4 spinal levels. Inclusion criteria were age>18, ASD, and completed patient surveys. Preoperative expectation survey included expectation of surgical outcome, expectation of pain reduction, likelihood of complications, and duration of postoperative recovery. Relationship between patient expectation of pain reduction and postoperative satisfaction was assessed.

Results: Thirty-two patients underwent operative management of ASD (mean age 67; 14 males, 18 females; 15 Open, 17 minimally invasive surgery) who met the inclusion criteria. In their preoperative survey, 66\% of patients expected highly successful surgery while $25 \%$ had moderate expectation of complications. Patients expected on average $71 \%$ reduction in back pain (range:42-100\%) and 68\% reduction in leg pain(range:0$100 \%)$. There was a moderate negative correlation between expectation of back pain reduction and postoperative satisfaction $(r=-0.4)$. There was moderate/high positive correlation between postoperative ODI and patient satisfaction $(r=0.5)$.

Conclusion: Majority of patients undergoing deformity surgery expected successful outcomes with significant improvement in pain. However, there was a significant variability in the expected recovery time and likelihood of complications. Patients with expectation of good outcome (i.e. reduction in pain) had high postoperative satisfaction but this relationship was only moderate.Postoperative satisfaction was highly correlated with postoperative ODI. 
8:30 - 8:32 am

\section{Complications, Radiographic, and Surgical Outcomes of Rigid Cervical Scoliosis and Combined Coronal Deformity Correction}

Darryl Lau; Rushikesh Sanjeev Joshi BS; Alexander F Haddad BS; Vedat Deviren MD; Christopher P. Ames MD;

Introduction: Isolated cervical scoliosis is rare while concurrent kyphoscoliosis is more frequently seen in adult cervical deformity (ACD). Such multiplanar cases are challenging especially for rigid deformities requiring osteotomies.

Methods: A retrospective review of ACD patients who underwent osteotomy for deformity correction from 2010-2019 was performed. Inclusion criteria was cervical kyphosis $>20$ degrees and/or cSVA >4cm and cervical scoliosis of 5 degrees or more.

Results: 30 patients were included. Mean age was 61.1 years, and $63.3 \%$ of patients were female. $50 \%$ underwent three column osteotomies. Mean number of levels fused was $12.16 .7 \%$ underwent combined anterior-posterior approaches. Cervical preoperative and postoperative coronal and sagittal parameters were significantly improved: cSVA (6.0 vs. $4.5 \mathrm{~cm}, p<0.001)$, cervical scoliosis $(16.7$ vs. 5.3 degrees, $p<0.001)$ and thoracolumbar scoliosis (20.1 vs. 14.8 degrees, $p=0.02)$. T1-slope was similar (42.8 vs. 38.0 degrees, $\mathrm{p}=0.170)$. Mean ICU stay was 2.6 days and hospital stay was 8.5 days. Complication rate was $40.0 \%$ : surgical $(13.3 \%)$ and medical (33.3\%). New neurologic deficits were observed in 4 patients (13.3\%). Two patients $(6.7 \%)$ required 30 -day readmission. Postoperative infections were seen in 2 patients $(6.7 \%) .36 .7 \%$ of patient underwent reoperation. In patients with 2-year follow-up, mechanical complications were observed in 8 patients $(26.7 \%)$.

Conclusion: Excellent radiographic correction of fixed cervical kyphoscoliosis can be achieved with posterior based osteotomies. Neurologic and surgical complication rates are low. But medical complication rate is high. This highlights careful selection and preoperative optimization of surgical candidates. 
8:32 - 8:34 am

\section{Risk Factors for Intensive Care Unit and Hospital Stay Following Osteotomies for Severe Cervicothoracic Deformity}

Rushikesh S. Joshi BS; Darryl Lau; Alexander F. Haddad BS; Vedat Deviren MD; Christopher P. Ames MD

Introduction: Correction of fixed cervicothoracic deformities can be associated with high complication rate and result in extended intensive care unit (ICU) and hospital stays.

Methods: A retrospective review of adult cervical deformity patients that underwent posterior based osteotomies for deformity correction from 2010-2019 was performed. Inclusion criteria was cervical kyphosis $>20$ degrees and/or cervical sagittal vertical axis (cSVA) $>4 \mathrm{~cm}$. Multivariate analyses was used to identify risk factors for hospital and ICU LOS.

Results: A total of 108 patients were included. Mean age was 65.5 years, and $66.0 \%$ of patients were female. $51.9 \%$ underwent three column osteotomy and $48.1 \%$ posterior column osteotomy. Overall, cervical and select thoracolumbar preoperative and postoperative measures significantly improved: cSVA (6.0 vs. $4.3 \mathrm{~cm}$, $\mathrm{p}<0.001$ ), cervical lordosis ( 8.2 vs. $-5.3 \mathrm{~cm}, \mathrm{p}<0.001)$, cervical scoliosis (6.5 vs. 2.2 degrees, $\mathrm{p}<0.001)$, t1 slope (40.2 vs. 34.5 degrees, $p<0.001$ ), thoracic kyphosis (54.4 vs. 46.4 degrees, $p<0.001$ ), lumbar lordosis (49.9 vs. 45.8 degrees, $p=0.003$ ) and thoracolumbar scoliosis (13.9 vs. 11.1 degrees, $p=0.009$ ). Overall ICU and hospital LOS were 2.8 days and 7.9 days, respectively. Multivariate analysis including patient characteristics and surgical parameters identified female sex as an independent factor associated with longer ICU (3.0 vs. 2.4 days, $p=0.018$ ) and hospital ( 8.3 vs. 7.3 days, $p=0.041)$ LOS. Greater number of levels fused (12 or more levels) was also independently associated longer ICU (3.5 vs. 1.9 days, $p=0.008)$ and hospital $(9.4$ vs. 6.2 days, $p=0.003$ ) LOS.

Conclusion: ICU and hospital LOS is significantly increased in patients undergoing cervicothoracic deformity correction for female patients and when 12 or more levels are fused. Additional multicenter prospective studies will be needed to validate our findings. 
8:34 - 8:36 am

\section{Neurologic Outcomes and Use of Neuromonitoring for Posterior Based Three Column Osteotomy for Cervicothoracic Deformity}

Darryl Lau; Cecilia L. Dalle Ore BA; Alexander F. Haddad BS; Vedat Deviren MD; Christopher P. Ames MD

Introduction: Correction of severe fixed cervical and cervicothoracic deformities can result in relatively high rates of neurological deficits. The performance measures of modern intraoperative neuromonitoring (IONM) during cervicothoracic three column osteotomy $(3 \mathrm{CO})$ have yet to be examined in detail.

Methods: We performed a retrospective review of a single surgeon experience from 2011 to 2018 of all patients who underwent posterior based $3 \mathrm{CO}$ in the cervicothoracic spine for deformity correction. Medical and neuromonitoring records were reviewed.

Results: 49 patients were included: 31 PSO and 18 VCR. Mean age was 61.1 years and $44.9 \%$ were male. $65.3 \%$ were myelopathic and $30.6 \%$ had preop weakness. Preoperative and postoperative measures were cSVA (6.8 and $3.7 \mathrm{~cm}, \mathrm{p}<0.001)$, lordosis ( 0.0 and 9.1 degrees, $p=0.030$ ), and T1-slope (49.8 and 36.7 degrees, $p<0.001)$. Complication rate was $44.9 \%$ and neurological deficit rate was $20.4 \%$. When stratified by osteotomy level, there was significantly higher rates of neurological deficits at C7 and T1: C7 (37.5\%), T1 (37.5\%), T2 (20.0\%), T3 (15.4\%), T4 (0.0\%) ( $p=0.023)$. C7 and T1 level deficits were most consistent with single nerve root weakness; C8-hand intrinsic most common followed by C7 triceps. There were 8 NM signal changes with 3 true positives and 7 false negatives. Performance measures were: accuracy $75.5 \%$, PPV $37.5 \%$, NPV $82.9 \%$, sensitivity $30.0 \%$, and specificity $87.2 \%$. In patients with new neurological deficits $42.9 \%$ recovered, $14.3 \%$ improved, and $28.6 \%$ were unchanged at 2 -year follow-up.

Conclusion: Neurologic complication rates are high following $3 \mathrm{CO}$ for cervicothoracic deformity correction. $3 \mathrm{CO}$ at $\mathrm{C} 7$ and $\mathrm{T} 1$ have the highest rates of neurological complication. IONM have modest performance in predicting postoperative deficits, but is a critical tool. Large multicenter prospective studies are is warranted. 
8:36 - 8:38 am

\section{Influence of Approach on Segmental Lordosis and Spinopelvic Correction with Lumbar Interbody Fusion: A Meta-Analysis and Systematic Review}

Jennifer Mao MBA; Jeffrey P. Mullin MD, MBA; Muhammad Waqas MBBS, FCPS; Tim O'Connor MD; Asham Khan MD; Felix Chin; John Pollina MD

Introduction: Lumbar interbody fusions (LIF) are used for the stabilization of painful motion segments, providing indirect decompression, and restore lumbar lordosis in corrective deformity surgery. LIFS can be performed from an anteriorly (ALIF), transforaminal (TLIF), and lateral (LLIF/DLIF/XLIF) approach. Studies have reported the change in spinopelvic parameters. Although many reports spinopelvic parameters, the degree of correction across approaches have yet to be reported.

Methods: A systematic review was conducted based on PRISMA guidelines of English articles from 20092019 from EMBASE, PUBMED, and Cochrane databases. Lumbar lordosis, segmental lordosis (SL), disc height (DH), pelvic tilt, pelvic incidence, sacral slope was extracted. SPSS (V26) and R metanalysis tool pack were used for data analysis. Forest plots were generated to visualize the effect sizes, study heterogeneity. Qualitative assessment was conducted via MINORS score.

Results: Qualitative and Quantitative analysis was conducted on 30 studies $(\mathrm{n}=762)$, with mean follow-up time of $36.6 \pm 22.1$ months. We showed significant improvement in SL at postop for ALIF and Lateral. Lateral approaches provided $10^{\circ}$ greater correction. For ALIF, these improvements were also seen at follow-up. SL improved significantly at follow up. ALIF, when compared with TLIF provided $\sim 10^{\circ}$ greater SL. DH increased post-surgically with ALIF, TLIF, and lateral. Specifically, ALIF and Lateral approaches increased DH by $41 \%$ \& $57 \%$ greater than TLIF. Postsurgical, ALIF and Lateral provide the greatest correction of LL. All approaches significantly decreased VAS Back pain score, and Significant improvement can be seen in VAS leg scores in ALIF and TLIF. The greatest effect towards improvement of ODI was at final follow-up with TLIF approach.

Conclusion: Although all approaches demonstrated significant improvement in LL TLIF provided the least. Lateral approaches provided comparable correction in LL as ALIF. As a corollary DH improved the greatest in ALIF and Lateral approaches. All approaches provided adequate and comparable improvement in VAS back pain. 
8:38 - 8:40 am

\section{Multilevel Posterior Column Osteotomies (MPCO): Technique Evaluation for Lumbar Sagittal Realignment in Unfused Discs}

Joseph A. Osorio MD, PhD; James Lin; Meghan Cerpa; Lawrence Lenke

Introduction: MPCOs are a useful technique, but the potential for mobility and correction of a spinal segment is contingent onlack ofcircumferential fusion. Therefore with unfused disc spaces, this techniquehasthe ability to aggregate large amounts of lordosis segmentally and can be utilized for revision surgeries as an alternative to 3-column osteotomies. We analyzed adult spinal deformity (ASD) patients with regional and/or global sagittal malalignment who had MPCOs performed for sagittal correction.

Methods: 98 lumbar levels (20 ASD patients) treated with post.-only surgery were analyzed. $70 \%$ were revision surgeries. Five sagittal plane radiographs were analyzed for each pt: preop standing, preop supine, intraop pre-rod insertion, intraop post-rod insertion, and postop standing. Cobb angles were measured segmentally. Lumbar levels were separated by operative invasiveness: prone pt. positioning, PCO, and PCO with TLIFs. The mean preop SVA was $7.2 \mathrm{~cm}$ (0.1-23.3) and mean PI-LL mismatch was 30 deg. (1 - 84). Ttests and ANOVA statistical analysis were used.

Results: 18 patients underwent MPCO to correct overall sagittal alignment. The t-test for revision vs primary: showed no statistical difference detected for intraop LL; $n=18 p=0.09$. ANOVA analysis comparing means of in situ correction of prone positioning alone vs. PCO vs. PCO with TLIF showed statistical difference when evaluating three modes of operative invasiveness: $p<0.0001$ (Table 1). All pts. had normalized regional/global sagittal plane realignment with significant difference in SVA $(7.2 \mathrm{~cm}(0.1-23.3)$ vs. $0.78 \mathrm{~cm}(-4.8$ to 5.26$)$, $\mathrm{p}<0.05)$; and PI-LL mismatch (30 deg. $(1-84)$ vs. $11(1-31), p<0.05)$ preop to postop respectively.

Conclusion: MPCO technique significantly restored sagittal correction in both the primary and revision ASD patients. Positioning, PCO, and PCO with TLIFs when utilized in multilevel sequential approach adequately restored sagittal balance in all patients. 
8:40 - 8:42 am

\section{Age is the Best Predictor for Length of Stay and Opioid Use Amongst Patients Undergoing Deformity Correction Surgery}

Emmanuel Adeyemo; Umaru Barrie; Salah G. Aoun MD; Madelina Nguyen; Olatunde Badejo; Zachary Christian; Kristen M. Hall BS; Valery Peinado Reyes PA; David L. McDonagh MD; Carlos A. Bagley MD

Introduction: Opioid medication has a central role in the management of postoperative pain medication but can lead to serious short- and long-term complications. During the current opioid epidemic, it has become imperative to identify factors putting patients at increased risk of increased opioid consumption following surgery.

Methods: Patients undergoing adult deformity correction surgery between November 2016 and February 2019 were included in this study. The total amount of postoperative opioid use was computed using Oral Morphine Equivalents (OME) units. Patients were divided into quartiles based on opioid consumption. Age, length of stay, and functional outcomes were then analyzed.

Results: Ninety-seven patients were included in the analysis. There was no statistical difference in the number of segments fused across all groups. The mean total opioid consumption for all patients was $276.5 \mathrm{mg}$ with a median of $264.4 \mathrm{mg}$. Mean consumption was $73.28 \mathrm{mg}, 204.82 \mathrm{mg}, 315.90 \mathrm{mg}$, and $538.86 \mathrm{mg}$ in the $1^{\text {st }}, 2^{\text {nd }}, 3^{\text {rd }}$, and fourth quartiles respectively. The average age of patients in group 4 was lower than all other groups. The average length of stay was also longer in group 4 compared to the other 3 groups ( $P$-value $<0.0001)$.

Conclusion: Younger patient age and longer hospital length of stay are associated with increased opioid consumption in patients undergoing spine deformity surgery. 
8:42 - 8:44 am

\section{Functional Status and Comorbidity Burden Are Predictors of Postoperative Complications in Adult Spinal Deformity Surgery}

Jianning Shao BA; Arbaz Momin; Jonathan J. Rasouli MD; Jacob Enders BSE; Edward C. Benzel MD; Michael P. Steinmetz MD

Introduction: Adult spinal deformity (ASD) surgery is associated with high complication rates, and frail patients are especially susceptible to postoperative complications. However, frailty has been loosely defined, with age often utilized as a proxy.

Methods: We retrospectively reviewed all patients who underwent ASD surgery at our institution during the past 20 years and collected data on patient demographics, frailty measures including Clinical Frailty Index (CFI), Charlson Comorbidity Index, modified frailty index ( $\mathrm{mFI})$, and preoperative Karnofsky Performance Scale (KPS), and complications. Logistic regression was performed to determine the best-fit model for predicting complication development.

Results: 600 patients were included in the final analysis: $198(35.6 \%)$ males and $358(64.4 \%)$ females. The average age was $59.8 \pm 16.7$ years, and the mean $\mathrm{CFI}, \mathrm{CCl}$, and $\mathrm{mFI}$, were $4.19 \pm 0.96,2.92 \pm 1.98$, and 0.12 \pm 0.10 . Median preoperative KPS was 70 . Logistic regression analyses found preoperative KPS $(p=0.002)$ and preoperative pelvic tilt angle $(p=0.026)$ to be significant predictive factors for $\mathrm{PJK}$, while $\mathrm{CCl}(p=0.030)$ and preoperative pelvic tilt angle $(p=0.006)$ were identified as significant predictors of all other postoperative complications. Of note, age was not found to be a significant predictor of complications $(p=0.22)$.

Conclusion: Preoperative functional status and comorbidity burden are important predictors of complications following ASD surgery. Age insufficiently informs this risk; rather, functional status and comorbidity burden in conjunction with preoperative pelvic tilt are more accurate predictors of complication development following ASD surgery. 


\section{8:44 - 8:46 am}

\section{Bone Morphogenetic Protein Use in Adult Thoracolumbar Deformity Surgery: Impact on Reoperation Rate and Cancer Risk}

Kunal Varshneya; Martin N. Stienen MD; Zachary A. Medress MD; Victor E. Staartjes; Atman Desai MD, MA; Gordon Li MD; Corinna C. Zygourakis MD; Jon Park; John K. Ratliff MD, FACS; Anand Veeravagu MD

Introduction: Bone morphogenetic protein (BMP) has been shown to stimulate bone growth and improve fusion rates in spine surgery. However, there is controversy regarding the impact of BMP on postoperative outcomes, reoperation rates, and risk of developing cancer in patients undergoing surgery for thoracolumbar deformity correction.

Methods: We queried the MarketScan database in order to identify patients who underwent ASD surgery from 2007-2016. Patients were then stratified into whether or not BMP was used in the index operation. Propensity score matching (PSM) was then utilized to mitigate intergroup differences between BMP and non-BMP patients. Patients under the age of 18 years and those with any prior history of cancer were excluded from this study. Baseline demographics and comorbidities, postoperative complication rates, short and long-term reoperation rates, and 5-year cancer risk were determined.

Results: A total of 33,610 patients met the inclusion criteria of this study, of which $n=1759(5.2 \%)$ received BMP during their index deformity operation. Patients in the BMP cohort experienced a higher rate of postoperative complications within 90 -days of surgery than control patients $(29.0 \%$ vs $25.1 \%, p<0.05)$. Patients receiving BMP during the index operation had lower rates of any revision surgery than control patients at 90 days $(8.2 \%$ vs $11.6 \%, p<0.05), 6$ months $(10.2 \%$ vs $13.2 \%, p<0.05)$, 1 year $(12.1 \%$ vs $15.2 \%$, $\mathrm{p}<0.05)$ and 2 years $(13.9 \%$ vs $18.0 \%, p<0.05)$. Overall risk of developing cancer was similar between the two cohorts (control: $22.7 \%$, BMP 22.4\%).

Conclusion: The use of BMP during ASD led to slightly higher immediate postoperative complications, reduced reoperations, and did not increase the risk of developing a malignancy. Further prospective studies should be undertaken to determine the impact of high and low dose BMP on outcomes for adult spinal deformity. 
8:46 - 8:48 am

\section{Efficacy and Safety of IV TXA in Reducing Blood Loss and Transfusions in Thoracolumbar Fusion}

Alexandre B. Todeschini MD; Benjamin G. McGahan MD; Alberto Uribe; Marco Echeverria-Villalobos; Juan Fiorda-Diaz; Mahmoud Abdel-Rasoul; Asad Akhter MD; Liesl Close; Benjamin McGahan; Andrew Grosscach; Stephanus Viljoen MD; Sergio Bergese

Introduction: Blood loss is potentially a major complication of spine surgery. Transexamic acid (TXA) is an anti-fibrinolytic that has been widely used to reduce blood loss in cardiac and orthopedic surgery.

Methods: Single-center, retrospective, nonrandomized, observational study of subjects who underwent multilevel ( $\geq 4$ ) spine fusion surgery who received or did not receive IV TXA between 2016 and 2018.

Results: 134 subjects, 88 (65.7\%) did not receive IV TXA (control group) and 46 (34.3\%) received IV TXA (TXA Group). The use of TXA was significantly higher in subjects with $\geq 7$ levels fused compared to the 4-6 level group ( $p<0.0001)$. The patients in the control group with $\geq 7$ levels fused had $1212.12 \mathrm{ml}$ greater EBL on average than patients in the TXA group with $\geq 7$ levels fused $(95 \% \mathrm{Cl}: 298.6$ to 2125.64 ; $p$-value $=0.0097)$. Also, the control group with $\geq 7$ levels fused received 2.26 more units of RBC on average than patients in the TXA group with $\geq 7$ levels fused (95\% Cl: 0.27 to 4.25 ; $p$-value=0.0265). There was not a difference in $E B L$ or mean RBC transfusions between the TXA and control groups among those with 4-6 levels fused.

Conclusion: The prophylactic use of intraoperative IV TXA could reduce intraoperative EBL and as well as RBC unit transfusion, especially in subjects undergoing spinal fusion greater than 7 levels. 
8:48 - 8:50 am

\section{Utility of Topical Tranexamic Acid in Spine Deformity Surgery: A Systematic Review and Meta-analysis}

Nida Fatima; John H. Shin MD

Introduction: Tranexamic acid (TXA) is an effective and commonly used hemostatic agent for perioperative conservation of blood in various surgical specialties.

Methods: We conducted a systematic review of the electronic databases using different MeSH terms from January 1970 to August 2019. Pooled and subgroup analysis was performed using fixed and random effect model based upon the heterogeneity $\left(\mathrm{I}^{2}\right)$.

Results: A total of 609 patients (tTXA: $n=258,42.4 \%$ ) from 8 studies were included. We found that there was a statistically significant difference in terms of (i) postoperative blood loss [Mean Difference (MD): $-147.1,95 \% \mathrm{Cl}$ : -189.5 to $-104.8, p<0.00001$ ], (ii) postoperative hemoglobin level (MD: $1.09,95 \% \mathrm{Cl}: 0.45$ to $1.72, p=0.0008$ ), (iii) operative time (MD: 7.47, 95\% Cl: 2.94 to 12.00, $\mathrm{p}<0.00001$ ), (iv) postoperative transfusion rate [Odds Ratio (OR): $0.39,95 \% \mathrm{Cl}: 0.20$ to $0.78, \mathrm{p}=0.007$ ], postoperative drain output (MD: $-184.0,95 \% \mathrm{Cl}:-222.03$ to 146.04, $p<0.00001$ ), and (v) duration of hospital stay (MD: $-1.14,95 \% \mathrm{Cl}:-1.44$ to $-0.85, \mathrm{p}<0.00001$ ) in patients treated with tTXA compared to the control group. However, there was no significant difference in terms of intraoperative blood loss $(p=0.13)$ and complications $(p=0.23)$ between the two comparative groups.

Furthermore, low dose $(250-500 \mathrm{mg})$ tTXA $(p<0.00001)$ reduced postoperative blood loss more effectively compared to high dose tTXA (1-3 g) $(p=0.001)$.

Conclusion: Our meta-analysis corroborates the effectiveness and safety of tTXA in spine deformity surgery. 
8:50 - 8:52 am

\section{Novel Radiographic Vertebral Bone Quality Score Predicts Symptomatic Adjacent Segment Disease Following Lumbar Fusion}

A. Karim Ahmed; Jeffrey S. Ehresman BS; Daniel Lubelski MD; Andrew Schilling; Zach Pennington BS; Ethan Cottrill BS; Julie McCracken; Majid Khan; Daniel M. Sciubba MD

Introduction: Poor bone quality is a known risk factor for symptomatic adjacent segment disease. The current standardhowever,DEXA, is not availale for most patients. As such, the current study aims to create a simple MRI-based score to evaluate bone quality and evaluate the degree to which it correlates with conventional DEXA scores and may predict adjacent segment disease.

Methods: Medical and radiographic record of patients who underwent elective lumbar fusion for degenerative conditions were obtained. Collected data included demographics (age, sex), body mass index (BMI), comorbidities, smoking status, symptoms, surgical indications, operative information, and radiographic information. Radiographic information included spinopelvic parameters, Pfirmann score, DXA, and VBQ score (T1 MRI). Patients who required reoperations after lumbar fusions due to symptomatic adjacent segment disease (assessed clinically and radiographically) were combined into a case group. A control group of equal size was then created using patients who did not require reoperation and this group was individually matched.Calculation of the VBQ score required T1-weighted MR images without contrast or inversion recovery.

Results: There was a significant difference in VBQ scores between the groups, with the Reoperation group having a higher VBQ score, indicating poorer bone quality $(p=0.030)$. A multivariable analysis was performed that included the previous significant variables (BMI, instrumented L1-L2, and VBQ score) to find the risk factors that independently predicted reoperation for symptomatic adjacent segment disease. This model found that both BMI $(p=0.039)$ and VBQ score $(p=0.030)$ were independent risk factors.

Conclusion: The novel VBQ score, based on T1-weighted MRI, successfully approximates bone density and predicts symptomatic adjacent segment disease following lumbar fusion. 
8:52 - 8:54 am

\section{Interim Analysis of a Prospective, Concurrently Controlled, Multi-Center FDA Study Results of Paraspinous Tension Band}

Alan T. Villavicencio MD; Sigita Burneikiene MD; Richard Sasso; Ivan Cheng; Todd Alamin; Louis Fielding; William F. Lavelle MD; S. Tim Yoon MD, PhD; Kee Kim; Hyun Bae MD; Jeffrey S. Fischgrund MD; Elizabeth Yu MD; Ravi Bains; Calvin Kuo; William C. Welch MD, FACS, FICS; Khalid A. Sethi; Michael Stauff; Reginald J. Davis MD, FAANS, FACS; Matthew Mermer; Harvinder S. Sandhu MD, MBA

Introduction: The LimiFlex device (Empirical Spine, San Carlos, CA) is a paraspinous tension band (PTB) stabilization option, which imparts segmental stabilization in the sagittal plane.

Methods: An interim analysis included all subjects who have reached a 12-month follow-up and compared perioperative, patient-reported clinical outcomes and adverse events.

Results: Seventy-two subjects reached $12 \mathrm{~m}$ follow-up: 38 and 25 in the PTB and TLIF groups, respectively. Mean $( \pm S D$ ) operative time, EBL, and length of hospital stay for PTB vs. TLIF patients were $112 \pm 27$ vs. $178 \pm 56$ minutes, $46 \pm 28$ vs. $250 \pm 164 \mathrm{~mL}$, and $0.7 \pm 1.7$ vs. $3.3 \pm 1.8$ days (all $p<0.0001$ ), respectively. Both groups demonstrated statistically significant improvements in VAS-leg/hip, VAS-back and ODI at 12-month follow-up (all $p<0.01$ ). The 15-point ODI improvement was achieved in $91 \%$ of PTB and $83 \%$ of TLIF patients. Five subjects $(17.2 \%)$ had durotomy and two subjects $(6.9 \%)$ were revised to three-level fusion due to symptom persistence in the TLIF group. Two subjects had surgical site infection $(4.7 \%)$ successfully resolved with medication, and one (2.3\%), out of two (4.7\%) spinous process fractures, was revised to fusion prior to discharge in the PTB group.

Conclusion: Avoiding making definitive conclusions based on interim data analysis, the 12-months clinical outcomes were comparable for both groups of patients. Further investigation will include a quantitative comparison between propensity-score matched groups with long-term follow-up. The PTB device may be a promising motion-preserving alternative to fusion for this patient population. 
8:54 - 8:56 am

\section{Indications for Surgical intervention from Adolescent to Adult idiopathic Scoliosis: An International Survey of Experts}

Jennifer Mao MBA; Jeffrey P. Mullin MD, MBA; Tim O'Connor MD; Alexander R. Vaccaro MD; Asham Khan MD; Justice O. Agyei MD; John Pollina MD

Introduction: It is not uncommon to delay Adolescent idiopathic scoliosis (AIS) into adulthood (AdIS). The decision for surgical intervention is challenging for this population, as the current understanding of curve progression has been extrapolated from adolescent studies. Scoliotic curves that $>30^{\circ}$ tend to progress into adulthood at an annual rate of 0.5 to $1^{\circ}$. With only a few studies reporting curve progression and rationale for surgical consultation, the optimal timing to treat this population is unknown.

Methods: A cross-sectional online survey was disseminated to the members of the Scoliosis Research Society. Suggestions and revisions were considered, and the final version was endorsed by the senior authors. Respondents were asked to indicate an annual rate of change that indicates immediate surgery, characterization of patients, rationale for consultation, change in a functional level, ASA grade at surgery, and comorbidities. Responses were analyzed using descriptive statistics and frequency analysis.

Results: 45 (3.9\%) participants from 16 countries accurately completed the study and were included in the analysis. The respondents saw both pediatric and adult patients. AdIS patients tend to have $1.82 \pm 1.69$ lifetime operations with an average age at surgery of $43.41 \pm 10.60$ years old and an ASA grade of $2.31 \pm 0.72$. The most frequent reason for consultation and surgery was due to Pain, quality of life, and cosmesis. Whereas, pain, the progression of the curve, and cosmesis were the main rationale for surgical intervention. Concomitant symptoms were common. The average rate of progression that indicated surgical intervention was $10.85^{\circ}$ $\pm 11.17^{\circ}$. This is significantly different than the normative average of $0.46^{\circ}(p<0.0001)$.

Conclusion: Although Curve progression is an indication for surgery, the natural rate of progression is significantly lower than the rate at which patients are receiving surgical intervention. Future studies are necessary to formulate a consensus guideline and identify indications for prophylactic corrective surgery for the prevention of worsening pain and progression in this population. 
8:56 - 8:58 am

\section{ACR vs PSO for Severe Sagittal Deformity: Clinical \& Radiologic Results of a Hyperlordotic Expandable Lateral Interbody}

Lara W. Massie MD; Hesham M. Zakaria MD; Mohamed Macki MD, MPH; Azam Basheer MD; Victor W. Chang MD; Frank LaMarca MD

Introduction: Anterior Column Reconstruction (ACR) is an increasingly utilized minimally invasive alternative to Pedicle Subtraction Osteotomy (PSO) for correction of sagittal plane deformity in patients with an available unfused lumbar disc.

Methods: Sixteen consecutive patients with sagittal plane imbalance (SVA $>10 \mathrm{~cm}$ or PI-LL mismatch/planned correction > 30) underwent either an ACR $(\mathrm{N}=11)$ using a hyperlordotic, expandable lateral interbody cage (ELSA-AL, Globus Medical, Audubon, PA) or PSO $(n=5)$.

Results: The ACR and PSO groups were similar at baseline: PI: 59 vs 57, $p=0.88$; PT: 32 vs 37, $p=0.64$; SVA: $12.72 \mathrm{~cm}$ vs $11.95 \mathrm{~cm} p=0.77$; segmental angulation (SA) 2.72 vs $2.75, p=0.99$. ACR produced significant improvements: LL 55.27, $p=0.0001$; Pi-LL 3.45, $p=0.0001$; PT 22.45, $p=0.02$; SVA 4.621 cm $p=0.0019$; segmental angle 25.09, $p<0.0001$. PSO also produced segmental lordosis which was not significantly different from the correction achieved by ACR ( 25 vs 29, $p=0.47$ ). In ACR, an average of 24.31 of lordosis was achieved at the index level, with an average cage expansion of 24.08. There was no significant difference in the number of levels fused posteriorly between the ACR and PSO groups ( 7 vs 8.75 levels, $p=0.175$ ) or length of surgery (375 mins vs 370 mins, $p=0.47$ ). However, there was significantly less blood loss in the ACR group $(311 \mathrm{~mL}$ vs $962 \mathrm{~mL}, p=0.0004)$ and shorter length of stay ( 7 days vs 11 days, $p=0.034)$. Pain scores were equivalent before ( 7 vs $7, p=0.18$ ) and after surgery $(A C R=2.8, P S O=3.2, p=0.39)$. Change in ODI was also significant for both groups, with no difference between groups at long term follow up $(A C R=-22.6, P S O=-17.7$, $\mathrm{p}=0.44)$.

Conclusion: ACR with the ELSA lateral interbody cage for significant sagittal deformity produced an equivalent degree of sagittal correction to PSO, equivalent improvement in disability and pain, with significantly less blood loss and shorter hospital length of stay. 
8:58 - 9:00 am

\section{Upright versus Supine Imaging is Superior to Flexion/Extension Xrays in Detection of Instability}

\section{Andrew Yew; Aleksey Ovchinnikov; Zoher Ghogawala MD, FACS}

Introduction: Dynamic instability is a primary indication for lumbar fusion surgery. However, the optimal method of detection of instability has not been established.

Methods: We performed an IRB approved retrospective single center study of consecutive patients who underwent a supine lumbar MRI within 3 months of an upright flexion/extension xray study from 1/1/2010 until $10 / 1 / 2019$. A trained research assistant performed measurements of the amount of anterolisthesis on a midsagittal T2 MRI, upright flexion, extension and neutral xrays utilizing the Meyerding method. In patients with multiple levels of spondylolisthesis, the level with the greatest anterolisthesis was selected. Instability was defined as $3 \mathrm{~mm}$ of displacement.

Results: 500 patients were eligible for inclusion, $48 \%$ male, $52 \%$ female. 432 patients were found to have spondylolisthesis. $18.7 \%$ demonstrated instability on flexion vs. extension radiographs versus $47 \%$ when comparing standing radiographs vs. MRI $(\mathrm{P}<0.001)$. There were only 7 cases that showed dynamic instability ( $\geq 3 \mathrm{~mm}$ ) when no change in spondylolisthesis was observed comparing standing radiographs to MRI. In 74/81 $(91.4 \%)$ cases of instability on flexion/extension, there was concordance between dynamic imaging and change in subluxation comparing standing images to MRI.

Conclusion: Flexion/extension xrays have a high false negative rate when attempting to assess for instability. Comparing upright xrays to a supine MRI appears to be the most sensitive method for eliciting instability. 
9:00 - 9:02 am

\section{Regional Heterogeneity in Sacropelvic Bone Densitometry is Amplified after Teriparatide Treatment}

Patrick Flanigan; Anthony L. Mikula MD; Soliman Oushy MD; Jeremy L. Fogelson MD; David W. Polly MD; Benjamin D. Elder MD, PhD

Introduction: Regional heterogeneity in sacropelvic bone quality can impact construct biomechanical stability for iliosacral screw and sacropelvic fixation. Hounsfield unit $(\mathrm{HU})$ analysis has been used previously as a surrogate for bone density, but changes in sacropelvic bone density following Teriparatide administration have not been assessed.

Methods: Retrospective chart review identified patients with Dual Energy X-ray absorptiometry (DEXA) and CT scans prior to initiation of and after completion of at least 6 months of teriparatide.

Results: Forty patients with a median age of 68 years were identified. Mean HUs of S1 I, SA, SB were different between groups $124 \pm 90.1 \mathrm{HU},-10.7 \pm 61.9 \mathrm{HU}$, and $168 \pm 55.4 \mathrm{HU}$, respectively $(\mathrm{p}<0.005)$. The mean HU at S2-I and S2-SB was not different (92.8 \pm 105 vs. 99.1 $\pm 72.1, p=0.74)$, however S2-SA (47.3 \pm 97.4$)$ was significantly lower than S2-I and S2-SB ( $p=0.003$ and $p=0.006$, respectively). After teriparatide, the HU of S2-I did not change, but S2-SB (99.1 vs. 130, $p=0.05)$, S1-I (124 vs. 165, $p=0.01)$, S1-SA (-10.7 vs. 3.68, $p=0.04)$, and S1-SB (168 vs. 189, $p<0.05$ ) increased. At the hip, DEXA t-scores increased from $-2.2 \pm 1.1$ to $-2.1 \pm 1.1$ and BMD increased from $0.72 \pm 0.14$ to $0.75 \pm 0.14$ following teriparatide.

Conclusion: We detected regional variation in sacropelvic bone densities using Hounsfield unit analysis; most regions increased in density after teriparatide treatment. Further analysis will determine how our results impact patient and screw trajectory selection. 
9:02 - 9:04 am

\section{Machine Learning Model to Predict Osteoporotic Spine with Hounsfield Units on Lumbar Computed Tomography}

Kyoung-Hyup Nam; Dong Hwan Kim; Byung Kwan Choi MD; In Ho Han

Introduction: Bone mineral density (BMD) is an important consideration during fusion surgery. Although dual X-ray absorptiometry (DEXA) is considered as the gold standard for assessing BMD, quantitative computed tomography (QCT) provides more accurate data in spine osteoporosis.

Methods: We reviewed 70 patients undergoing both QCT and conventional lumbar CT for spine surgery. The T-scores of 198 lumbar vertebra was assessed in QCT and the HU of vertebral body at the same level were measured in conventional CT by the picture archiving and communication system (PACS) system. A multiple regression algorithm was applied to predict the T-score using 3 independent variables (age, sex, and HU of vertebral body on conventional CT) coupling with T-score of QCT. Next, a logistic regression algorithm was applied to predict osteoporotic or non-osteoporotic vertebra. The Tensor flow and Python were used as the machine learning tools. The Tensor flow user interface developed in our institute was used for easy code generation.

Results: The predictive model with multiple regression algorithm estimated similar T-scores with data of QCT. HU demonstrates the similar results as QCT without the discordance in only one non-osteoporotic vertebra that indicated osteoporosis. From the training set, the predictive model classified the lumbar vertebra into two groups (osteoporotic vs non-osteoporotic spine) with $88.0 \%$ accuracy. In a test set of 40 vertebrae, classification accuracy was $92.5 \%$ when the learning rate was 0.0001 .

Conclusion: This study is a simple machine learning model applicable in the spine research field. The machine learning model can predict the T-score and osteoporotic vertebrae solely by measuring the HU of conventional CT, and this would help spine surgeons not to under-estimate the osteoporotic spine preoperatively. 
Spine Summit Meeting Abstracts, May 2021

9:04 - 9:06 am

\section{Predicting Determinants of Variation in Episode-of-care Bundled Payments for Adult Spinal Deformity Surgery}

Zachary A. Medress MD; Anand Veeravagu MD; John K. Ratliff MD, FACS; Atman Desai MD, MA; Corinna C. Zygourakis MD; Kunal Varshneya; Parastou Fatemi; Adrian Rodrigues BA; Stephen Skirboll MD; Jon Park MD, FRCS(C); Martin N. Stienen MD

Introduction: Episode-of-care bundled payments (EBP) combine preoperative, inpatient, and postoperative care reimbursements into a single bundled payment to promote care coordination and reduce costs. EBP have been introduced in simple spinal fusion and decompression operations but have not yet expanded to adult spinal deformity (ASD) surgery. Given the substantial resource utilization associated with ASD surgery, ASD may become a target for future iterations of bundled payment care improvement initiatives (BPCI).

Methods: We performed a retrospective, observational study using the Marketscan database to generate 30 , 60, and 90-day bundled payment projections for ASD surgery episodes of care. Multivariable analysis was performed to identify the impact of pre-operative, operative, and post-operative characteristics on ASD surgery bundled payment values.

Results: 10,862 operations met inclusion criteria. Average index hospitalization payment $=\$ 99,976$; mean total projected bundled payments were $\$ 163,752, \$ 167,108$, and $\$ 169,520$ for $30-$-, 60-, and 90-day bundles, respectively. On multivariable regression analysis, pre-operative characteristics including advanced age, osteoporosis, and baseline paraparesis significantly increased projected bundle payments. Technical aspects including a combined anterior/posterior surgical approach increased projected bundle payments; however, recombinant bone morphogenetic protein use did not affect projected bundled payments. Discharge to an inpatient rehabilitation facility was associated with a $15.8 \%(95 \%$ C.I. 10.2-21.8, p<0.005) increase in 90-day bundle value. Postoperative complications including hematoma, venous thrombo-embolism, infection, and neurologic injury significantly increased projected bundled payments.

Conclusion: We generated projections of 30-, 60-, and 90-day EBP for ASD surgery. Baseline preoperative characteristics, surgical approach type, discharge destination, and post-operative complications significantly impacted bundle values for 30-, 60-, and 90-day episodes of care. Based on these data, we predict that expansion of BPCI to include ASD surgery may have important long-term ramifications on patient selection, approach type, and practice patterns. 
9:06 - 9:08 am

\section{Age, BMI and Osteoporosis are More Predictive than Imaging for Adjacent Segment Re-Operation after Lumbar Fusion}

Nii Ankrah; Andrew Yew

Introduction: Adjacent segment disease is a common long-term complication following lumbar fusion. We aim to analyze the effect of segmental lumbar lordosis (LL) on the rates of adjacent segment disease and rates of revision surgery following lumbar fusion at adjacent level.

Methods: An IRB approved retrospective single center study was performed with patients who underwent index lumbar fusion surgery and/or revision surgery at Lahey from May 2012 to November 2018 utilizing the Lahey Lumbar Fusion registry. Inclusion criteria included having both pre- and postoperative upright xrays. Revision surgeries for which the index operation was performed at an outside hospital were excluded. Bivariate analysis was conducted on candidate variables and variables with $p<0.2$ were selected for multivariate logistic regression.

Results: 21/106 patients required reoperation. Age $\geq 65 y r s$ (OR 4.14, 95\% Cl $1.46-11.76, p=.008$ ), BMI $\geq 34$ (OR 1.13, 95\% Cl $1.04-1.23, p=.004$ ), and osteoporosis (OR 14, 95\% Cl $1.38-142.42, p=.03$ ) were independent predictors of re-operation in the multivariate analysis. Increased facet diastasis at fusion levels (OR $0.60,95 \% \mathrm{Cl} 0.42-0.85, \mathrm{p}=.004$ ) was associated with reduced reoperation rates. Change in segmental lumbar lordosis at the index operation level, rostral and caudal facet diastasis, vacuum discs, T2 hyperintensity in the facets were not a predictor of re-operation.

Conclusion: Age 65, BMI 34 and osteoporosis were independent predictors of adjacent segment reoperation. Change in segmental lordosis at the index level did not appear to affect reoperation rates. 
9:08 - 9:10 am

\section{Preventing Proximal Junctional Failure in Open Thoracolumbar Fusion Using Multilevel Transvertebral Stabilization Screws}

Ascher Kaufmann; Doris Tong; Guriqbal Bhullar; Matthew Bahoura BA; Chad F. Claus DO; Teck Mun Soo

Introduction: Acute Proximal junctional failure (PJF) following thoracolumbar fusion for adult spinal deformity (ASD) has a reported rate up to 38.5\%. Use of Multilevel Transvertebral Stabilization Screws (MLSS) may improve this outcome.

Methods: We reviewed the charts of consecutive ASD patients who underwent open thoracolumbar fusion ( $>3$ levels extending cephalad from T6) between 2011-2017. The standard thoracic screw placement was augmented by MLSS at the most cephalad level where the MLSS screw entered the pedicle from the inferolateral aspect following a superomedial trajectory, reaching the superior cortex at the anterior aspect of the pedicle. The widest and longest screw permitted was chosen to achieve the most superior vertebral fixation.

Confounder data were collected. PJF was divided into acute vs. chronic ( $<>>28$ weeks). PJF was defined as vertebral fracture of the upper instrumented vertebrae (UIV) or UIV+1, failure of UIV fixation, or proximal extension of fusion. Clinical and radiographic outcomes were collected during clinic follow-up or hospital presentation. Sensitivity analysis accounted for patients lost to follow up. Descriptive statistics were used.

Results: Forty-four patients were eligible. Seven patients did not reach $28 \mathrm{w}$ follow-up. The mode for UIV and lower instrumented vertebrae (LIV) were T2 (40.5\%) and S1 (32.4\%); mean operative and MLSS levels were $11.9 \pm 2.6$ and $3.32 \pm 0.6$ respectively. The average follow-up was $175.6 \pm 91.7$ weeks. Eighteen patients $(48.6 \%)$ suffered PJF and 2 (5.4\%) acute PJF and 16 (43.2\%) chronic. Average time to acute failure was $19.6 \pm 8.7$ weeks and chronic failure $91.1 \pm 46.3$ weeks. The PJF causes were UIV fixation failure (61.1\%) and extension of fusion (38.9\%). Sensitivity analysis estimated our PFJ range to be $40.9 \%-56.8 \%$.

Conclusion: Our acute PJF and revision surgery rate with MLSS is consistent with some of the lowest numbers reported. Further prospective investigation is warranted. 
9:10 - 9:12 am

\section{Anterior Lumbar Enhanced Recovery After Surgery (ERAS) for Adult Spinal Deformity: Case Series and Proposed Protocol}

John F. Burke MD, PhD; Taemin Oh MD, BA; Vivek Sudhakar; Darryl Lau; Charles Eichler; Aaron J. Clark MD PhD; Christopher P. Ames MD;

Introduction: Adult spinal deformity surgery often involves anterior lumbar interbody fusion (ALIF), which is often associated with postoperative ileus. Enhanced recovery after surgery (ERAS) protocols have been implemented in the general surgery literature to reduce post-operative ileus, however application of these protocols to adult spinal deformity surgery are limited.

Methods: We retrospectively identified all patients in one year who underwent ALIF for adult spinal deformity. We applied a formal definition of ileus to each patient's post-operative course, and compared the length of stay for patients with ileus to those without. We also trained a multivariate classifier to predict the occurrence of ileus.

Results: 47 patients were identified who underwent ALIF surgery for deformity correction. The rate of retrospectively defined ileus was $57.45 \%$, and $44.44 \%$ of patients with ileus were not recognized at the time of admission. Patients with ileus had a significant increase in the overall hospital length of stay $(p=0.042)$, and the length of stay was directly proportional to the number of days until return of bowel function $(p=0.001)$. Using a multi-variate classifier, the minimum value of the post-operative serum potassium had the most predictive power to detect ileus.

Conclusion: Post-operative ileus after ALIF for ASD is a significant cause of increased length of stay. We propose an ERAS protocol to prevent, identify, and treat post-operative ileus, which should decrease the overall cost of deformity surgery. 
9:12 - 9:14 am

\title{
Feasibility of Machine Vision-based Spinal 3-Dimensional Navigation in the Sacrum and Pelvis
}

\author{
Dan T. Chen; Albert Yee; Victor X. D. Yang MD, PhD, MSc, BSc
}

Introduction: The prevalence of intraoperative navigation in spinal surgery is increasing, with well established benefits in accuracy and safety. Optical topographical navigation using machine vision (MV) is a relatively novel technique that has been validated in spinal procedures involving the cervical and thoracolumbar segments with comparably more efficient workflow and similar accuracy to conventional navigation. There is a paucity of published literature on MV-based navigation for sacral-pelvic instrumentation.

Methods: This is a multicenter, retrospective review of consecutive spine surgery patients who underwent MVassisted spinal instrumentation involving distal fixation to the sacrum and/or pelvis. Standard posteriorly based midline incisions were performed in all cases and registration was performed on fixed bony landmarks in these segments. Planned screwed trajectories were compared against postoperative computed tomographic scans to measure translational and angular deviations. Case registration times and total number of registered points were collected and analyzed. Descriptive and inferential statistics were performed, the results were compared against known industry standards.

Results: A total of 200 cases were collected that utilized MV-based spinal navigation for spinal instrumentation involving distal fixation to the sacrum and pelvis. An average of 3259.11 points were successfully registered per case. The overall calculated registration accuracy is $0.2536 \mathrm{~mm}$, and is comparable to conventional navigation systems. On radiographic review, no sacral foraminal or canal violations were found, and all screw fixation remained appropriately within the inner and outer tables of the ilium. No iatrogenic neurovascular complications were found on clinical review.

Conclusion: Our retrospective review demonstrates that machine vision-based spinal navigation is a rapid and accurate alternative to traditional image guidance systems with proven patient safety in sacral and pelvic instrumentation. 
9:14 - 9:16 am

\section{Augmented Reality Precision and Accuracy for Percutaneous Pedicle Screw Insertion - A Multi Cadaveric Analysis}

Camilo Molina; Frank M. Phillips MD; Matt Colman; Majid Khan; Emanuele Orru; Kornelis Poelstra MD; Larry T. Khoo MD

Introduction: Augmented reality mediated spine surgery (ARMSS) is a minimally invasive novel technology that has the potential to increase the efficiency, accuracy, and safety of conventional percutaneous pedicle screw insertion methods. Visual 3-dimensional spinal anatomic and navigation images are directly projected onto the operators retina and superimposed over the surgical field, eliminating field-of-vision and shift to a remote display.

Methods: 5 cadaveric torsos were ARMSS instrumented with the XVISION platform (Augmedics; Chicago, IL) at levels ranging from T5 to S1 for a total of 113 total implants comprised of 93 pedicle screws and 20 jamshidi needles. Post-procedural computed tomography (CT) scans were graded by 2 independent neuroradiologists using the Gertzbein scale (GS A-E). Precision was calculated via superimposition analysis employing the Medical Image Interaction Toolkit (MITK; Heidelberg, Germany) to yield angular trajectory $\left({ }^{\circ}\right.$ ) and screw tip $(\mathrm{mm})$ deviation from the virtual navigated pedicle screw position versus the actual pedicle screw position on post-procedural CT imaging.

Results: The overall implant placement accuracy achieved via ARMSS was $99.1 \%$. Sacro-lumbar and thoracic accuracy was $100 \%$ and $98.3 \%$, respectively. Specifically, among all implants inserted, 112 were noted to be GS A or B (99.12\%) with only 1 medial GS C breach ( $>2 \mathrm{~mm}$ pedicle breach) in a thoracic pedicle at T6. Precision analysis of inserted pedicle screws yielded a mean screw tip linear deviation of $1.98 \mathrm{~mm}$ [99\% $\mathrm{Cl}: 1.74-2.21 \mathrm{~mm} ; \mathrm{p}=0.01]$ and a mean angular error of $1.29^{\circ}\left[99 \% \mathrm{Cl}: 1.11^{\circ}-1.46^{\circ} ; \mathrm{p}=0.01\right]$ from the projected trajectory. The latter data compare favorably to regulatory precision requirements mandating that linear and angular deviation be below $3 \mathrm{~mm}$ and $3^{\circ}$, respectively.

Conclusion: Percutaneous ARMSS pedicle implant insertion is a technically feasible, accurate, and highly precise method. 
9:16 - 9:18 am

\section{Prediction Calculator for Length of Stay and Nonroutine Discharge after Elective Spine Surgery}

Daniel Lubelski MD; Jeffrey S. Ehresman BS; James Feghali MD; Joseph Tanenbaum; Ali Bydon MD; Nicholas Theodore MD; Timothy F. Witham BS, MD; Daniel M. Sciubba MD

Introduction: Following spine surgery, delays in referral to rehabilitation facilities leads to increased length of hospital stay (LOS), increases costs, more risk of hospital acquired complications, and decreased patient satisfaction.

Methods: We retrospectively reviewed patients who underwent elective spine surgery for degenerative disease between January and June 2018. Demographic variables, insurance status, baseline comorbidities, narcotic use, operative characteristics, as well as postoperative length of stay and discharge disposition data were collected. Univariable and multivariable analyses were performed to identify independent predictors of LOS and discharge disposition.

Results: 257 patients were included. Mean age was 59 years, $46 \%$ were females, and $52 \%$ had private insurance vs $7 \%$ with Medicaid and $41 \%$ with Medicare. The most commonly performed procedure was lumbar fusion (31.9\%). Mean LOS after surgery was 4.8 days and $18 \%$ had prolonged LOS $>7$ days. Age, insurance type, marriage status, and surgical procedure were significantly associated with LOS and discharge disposition. The final model had an area under the curve of $89 \%$ with good discrimination. A web based calculator was developed: https://jhuspine1.shinyapps.io/length of stay calculator/

Conclusion: This study established a novel highly predictive pilot calculator to identify those patients most likely to be discharged to rehabilitation facilities and to predict LOS after spine surgery. With validation this tool may ultimately facilitate streamlining of the postoperative period to shorten LOS, optimize resource utilization, and improve patient care. 
9:18 - 9:20 am

\title{
Radiologic and One-year Clinical Outcomes in ALIF and TLIF
}

\author{
Ziev Moses MD; Seok Yoon Oh; Brian T. David PhD; Richard G. Fessler MD, PhD
}

Introduction: Single level lumbar degenerative disease is a major driver of morbidity and decreased quality of life. Transforaminal lumbar interbody fusion (TLIF) and anterior lumbar interbody fusion (ALIF) are two common surgical strategies used to treat symptomatic disease.

Methods: Retrospective chart review of 181 patients who underwent a single-level TLIF (127) or ALIF (54) over a 48-month period with at least one year follow up. Segmental lordosis and lumbar lordosis were measured on standing $x$-rays at early and delayed time points, in addition to a battery of outcome measures at regular intervals, including VAS-leg, VAS-back, ODI, SF-12 physical health, NRS-Leg, and SRS30.

Results: The mean study group age was 60.6 years (92 female; 89 male). There were no significant differences in age or sex between groups. EBL and length of stay were significantly higher in the TLIF group ( 169.5 cc vs 69.7 cc, $p<0.05 ; 3.7$ vs 2.9 days, $p<0.05)$. Complication rates were similar ( $9 \%$ ALIF and $6 \%$ TLIF). Changes in lumbar lordosis and segmental lordosis were significantly greater after ALIF $\left(3.9^{\circ}\right.$ vs $-0.5^{\circ} \mathrm{p}$ $<0.05,4.8^{\circ}$ vs $-0.9^{\circ}, p<0.05$ ) at delayed follow up imaging (mean time, $366+/-20$ days). At one year or greater, mean ODI score was lower in the TLIF group (26.3 vs $33.3, p<0.05)$, mean SF-12 physical health score was higher in the TLIF group (39.0 vs 32.6, $p<0.05$ ), and mean SRS-30 score was higher in the TLIF score (3.6 vs 3.1, p < 0.05).

Conclusion: This study suggests that while greater changes in lumbar lordosis and segmental lordosis occur following ALIF, HRQOL scores signified lower disability and higher physical health scores following TLIF at one-year. 
9:20 - 9:22 am

\title{
Coronal Balance in Degenerative Scoliosis Treated with Circumferential Minimally Invasive Fusion
}

\author{
Corey T. Walker MD; Jakub Godzik MD, MSc; Jay D. Turner MD, PhD; Juan S. Uribe MD, FAANS
}

Introduction: Circumferential minimally invasive anterior and lateral transpsoas interbody fusion (cMIS) can be used to address coronal plane deformity in patients with degenerative scoliosis.It remains unclear how well cMIStreats coronal plane malalignment.

Methods: A retrospective evaluation of patients with degenerative scoliosis (Cobb $\left.>20^{\circ}\right)$ treated with cMIS (lateral transpsoas fusion combined in some cases with anterior lumbar interbody fusion and percutaneous pedicle screw fixation) was performed. Patients' coronal balance was categorized according to the Qui classification as A (balanced), B (C7CSVL $>3 \mathrm{~cm}$ towards the concavity of the major curve) or C (>3cm towards the convexity).

Results: 40 patients were treated with cMIS approaches (mean age - 68yrs, Male/Female - 13/27). 17 (43\%) were imbalanced pre-operatively, 6 as $B$ type and 11 as $C$ type. All A type remained balanced post-operatively. 5 of $6 \mathrm{~B}$ patients and 6 of $11 \mathrm{C}$ patients improved to A with surgery. C7SVL was not different pre- and postoperatively ( 2.4 vs $1.9 \mathrm{~cm}, p=0.15)$. Significant improvements in the major curve $\left(28^{\circ}\right.$ vs $\left.15^{\circ}, p<0.001\right)$, fractional curve $\left(13^{\circ}\right.$ vs $\left.8^{\circ}, p<0.001\right)$, secondary curve $\left(15^{\circ}\right.$ vs $\left.9^{\circ}, p=0.001\right)$, L4 tilt $\left(14^{\circ}\right.$ vs $\left.8^{\circ}, p<0.001\right)$ and L5 tilt $\left(8^{\circ}\right.$ vs $\left.6^{\circ}, p=0.02\right)$ were seen. Surgical intervention was associated with improvements in ODI, VAS back and VAS leg (all $p<0.001)$. The patients with post-operative coronal malalignment had significantly less improvement in ODI with surgical intervention ( $\triangle \mathrm{ODI} 16$ vs $26, \mathrm{p}=0.03$ ). The patients with post-operative sagittal malalignment (SVA $>5 \mathrm{~cm}$ ) had equivalent improvements ( $\triangle$ ODI 20 vs $24, p=0.56$ ).

Conclusion: cMIS deformity surgery in patients with degenerative scoliosis is successful at treating coronal imbalance in the majority of patients. Patients with $C$ type imbalance remain difficult to treat. Failure to restore coronal alignment results in less improvement in quality of life metrics. 
9:22 - 9:24 am

\section{The Effect of Concurrent Sagittal and Coronal Imbalance Following Three Column Osteotomies for Thoracolumbar Deformity}

Darryl Lau; Alexander F. Haddad BS; Rushikesh S. Joshi BS; Vedat Deviren MD; Christopher P. Ames MD

Introduction: Rigid multiplanar deformities such as concurrent sagittal and coronal deformity cases are challenging and may require an asymmetric three-column osteotomy (3CO).

Methods: We performed a retrospective review of a consecutive ASD patients who underwent thoracolumbar $3 \mathrm{CO}$ for sagittal deformity from 2006 to 2019 . Coronal imbalance was defined as CSVL $4.0 \mathrm{~cm}$ or more. Perioperative and 2-year complications were compared: pure sagittal vs. combined sagittal-coronal cases. Multivariate analysis was used.

Results: 390 patients were included: 338 sagittal and 52 combined. Mean age was 64.6 years and $65.1 \%$ were female. Preoperative measurements between the groups were as follows: SVA $10.7 \mathrm{vs} .13 .0 \mathrm{~cm}$ $(p=0.042)$, CSVL 1.2 vs. $6.1 \mathrm{~cm}(p<0.001)$, PI 64.8 vs. 60.0 degrees $(p=0.041)$, PT 28.9 vs. 36.5 degrees $(p<0.001)$, and LL -14.5 vs. -12.9 degrees $(p=0.778)$. There were no significant differences in rate of overall (33.7\% vs. $42.3 \%, p=0.227)$, neurologic ( $5.9 \%$ vs. $3.9 \%, p=0.547)$, medical $(20.7 \%$ vs. $25.0 \%, p=0.482)$ or surgical $(6.5 \%$ vs. $11.5 \%, p=0.191)$ complications. Combined patients had significantly longer hospital (10.8 vs. 8.3 days, $p=0.002$ ) and ICU stays ( 3.1 vs. 2.3 days, $p=0.047$ ). CSVL $>4 \mathrm{~cm}$ was independently associated with an increase in LOS ( $p=0.030) .66 .1 \%$ of patients had 2-year follow-up. Rate of reoperation for mechanical complications was similar at 2 -year follow-up $(34.5 \%$ vs. $29.2 \%, p=0.600)$.

Conclusion: Concurrent coronal imbalance does not impact perioperative and mechanical complication rates following thoracolumbar 3CO. Patients with combined deformities stay in the ICU and hospital longer. 
9:24 - 9:26 am

\section{Defining Complication and Neurologic Deficit Rates Following Posterior Based Osteotomies for Cervicothoracic Deformity}

Darryl Lau; Vedat Deviren MD; Christopher P. Ames MD

Introduction: Correction of severe cervicothoracic deformity can be associated with significant morbidity. Three-column osteotomy (3CO) and posterior column osteotomy (PCO) complication profiles and risk factors has yet to be defined.

Methods: A retrospective comparison of complications between posterior $3 \mathrm{CO}$ and PCO was performed in a single surgeon experience from 2011 to 2018. Patients had cSVA of $>4 \mathrm{~cm}$ and underwent cervicothoracic deformity correction. Multivariate analysis was utilized.

Results: 95 patients were included: $493 \mathrm{CO}$ and 46 PCO. 12 of PCO had anterior releases. Mean age was 63.2 years and $60.0 \%$ were female. Preoperative and postoperative parameters: cSVA $(6.2$ and $3.5 \mathrm{~cm}$, $p<0.001$ ), cervical lordosis (-6.8 and 7.5 degrees, $p<0.001)$, and T1-slope (40.9 and 35.2 degrees, $p=0.026$ ). Complication rate was $37.9 \%$ and neurologic deficit was $16.8 \%$. Surgical and medical complication rates were $17.9 \%$ and $23.2 \%$. Surgical and neurologic complication rate was higher with $3 \mathrm{CO}$ but was not significant $(42.9 \%$ vs. $32.6 \%, p=0.304,18.4 \%$ vs. $10.9 \%, p=0.303$, and $20.4 \%$ vs. $13.0 \%, p=0.338)$. Medical complication rates were similar $(23.9 \%$ vs. $22.4 \%, p=0.866)$. Independent risk factors for surgical complication were male gender (OR 10.88, $p=0.014$ ) and cSVA >8 cm (OR 10.36, $p=0.037$ ). Anterior-posterior surgery was independently associated with medical complications (OR 10.30, $p=0.011)$. Kyphosis $>20$ degrees was an independent risk factor for neurological deficit (OR 2.08, $p=0.011)$.

Conclusion: Complication and neurologic deficit rates are high in following correction of cervicothoracic deformity. Preoperative cSVA $>8 \mathrm{~cm}$ and kyphosis $>20$ degrees are independent risk factors. 
9:26 - 9:28 am

A Prospective Analysis of Minimally Invasive Surgery for Adult Spinal Deformity: A Multicenter Study

Gregory M .Mundis; Jakub Godzik MD, MSc; Paul Park MD; Kai-Ming G. Fu MD, PhD; Stacie Tran BS, MPH; Juan S. Uribe MD, FAANS; Michael Y. Wang MD, FAANS; Khoi D. Than MD; David O. Okonkwo MD, PhD; Adam S. Kanter MD; Pierce D. Nunley MD; Neel Anand MD; Richard G. Fessler MD, PhD; Dean Chou MD; Robert Eastlack MD

Introduction: Traditional surgery for adult spinal deformity (ASD) is effective but results in exposure related morbidity. Minimally invasive spine surgery (MISS) can minimize this morbidity but high-level evidence for efficacy is lacking. This study presents the first prospective, multi-center investigation of MISS for ASD.

Methods: Patients $\geq 18$ years old with $\geq 1$ criteria: coronal curve $(C C) \geq 20^{\circ}, S V A>5 \mathrm{~cm}, \mathrm{PT}>25^{\circ}$, thoracic kyphosis (TK) $>60^{\circ}$ were included. Patients had MIS surgery including interbody work: TLIF, LLIF, ALIF, ACR and percutaneous posterior fixation. 64 patients with minimum 1-year follow-up were included. Subgroup analysis of 26 with $\geq 1$ Schwab ++ modifier was performed to evaluate those with more severe deformity (SD). Radiographic and clinical outcomes included VAS, ODI and SRS-22.

Results: Mean age was 67.5 years. Mean levels instrumented 4.3, EBL $366.5 \mathrm{cc}$, and LOS 6.9 days. Significant $(p<0.05)$ improvements in SVA $(54 \mathrm{~cm}$ to $33.3 \mathrm{~cm}), \mathrm{PI}-\mathrm{LL}\left(13.6^{\circ}\right.$ to $\left.6.3^{\circ}\right), \mathrm{CC}\left(22.9^{\circ}\right.$ to $\left.15.5^{\circ}\right)$ were observed. Significant $(p<0.05)$ improvements in ODI (44.9 to 27.8$)$, SRS (2.8 to 3.7), VAS back (7.1 to 2.8$)$ and VAS leg (6 to 3.1) were noted. Complications occurred in 21 patients (32.8\%), 13 (20.3\%) major, including 5 $(7.8 \%)$ neurologic, with a 1 year reoperation rate of $15.6 \%(n=10)$. In SD patients $(n=28)$, similar improvements in SVA $(94.5 \mathrm{~cm}$ to $45.9 \mathrm{~cm}), \mathrm{PI}-\mathrm{LL}\left(25.6^{\circ}\right.$ to $\left.9.5^{\circ}\right)$, PT $\left(26.9^{\circ}\right.$ to $\left.22.7^{\circ}\right), \mathrm{CC}\left(20.4^{\circ}\right.$ to $\left.12.8^{\circ}\right)$, ODI (49.8 to 30.8$)$, SRS-22 (2.8 to 3.5), VAS back (7.4 to 3.2), VAS leg (5.8 to 3.7) were found $(p<0.05)$. Mean age was 69.5 years and mean levels treated 5.1, EBL $464.6 \mathrm{cc}$, and LOS 7.8 days. Complications occurred in 14 (53.8\%), 8 $(28.6 \%)$ major, including $2(7.1 \%)$ neurologic with 6 requiring a reoperation $(21.4 \%)$.

Conclusion: MIS for ASD resulted in meaningful symptomatic improvement. Complication rates are similar to historic norms with a fairly high reoperation rate at1year. Longer follow up will be necessary to evaluate the durability of this approach in the treatment of adult deformity. 
9:28 - 9:30 am

\section{Patient Satisfaction after Surgical Management of Adult Spinal Deformity: MIS vs. Open Techniques}

W. H. Andrew Ryu BSc, MSc, MTM, MD; Michael Cheong; Ziev Moses; Brian T. David PhD; Ricardo B. V. Fontes MD, PhD; Richard G. Fessler MD, PhD

Introduction: Patient satisfaction has become an important variable in assessing outcomes after spine surgery with the growing emphasis on patient reported outcomes(PRO). While minimally invasive surgery(MIS) techniques have become increasingly popular due to reduction in perioperative complications over open deformity surgery, it is unclear if patient reported postoperative satisfaction differ between the two surgical approaches.

Methods: Retrospective chart review was performed on patients who underwent deformity correction surgery between 2016-2018 involving at least 4 spinal levels. Inclusion criteria were age>18, adult spinal deformity(ASD), and completed PRO surveys postoperatively. Patient satisfaction was rated using visual analog scale out of 50 with 0 representing "delighted" and 50 representing "not satisfied". Patient demographics, clinical data, and PRO scores were compared between the open and MIS groups.

Results: Forty-five patients underwent operative management of ASD(24 Open vs. $21 \mathrm{MIS})$ who met the inclusion criteria. Preoperative Oswestry Disability Index(ODI) scores were similar between the two groups(52.3 vs.48.5; $p=0.22$ ). The Open deformity group involved a significantly higher number of spinal levels compared to the MIS deformity group(10 vs 6; $p=0.00001)$ with greater intraoperative blood loss $(1833 \mathrm{ml}$ vs. $653 \mathrm{ml} ; \mathrm{p}=0.001)$. Both groups had 2 cases of intraoperative complications. Postoperative ODI did not differ between the Open group and the MIS group at 6-month and 12-month follow-up(32.4 vs 30.6, $p=0.36 ; 36.0$ vs. $26.9, p=0.10)$. Furthermore, patient satisfaction did not differ between the Open group and the MIS group at 6month and 12-month follow-up(10.4 vs. $13.1, p=0.19 ; 10.6$ vs. $12.2, p=0.38)$.

Conclusion: Patient reported outcomes after open deformity and MIS deformity surgery reflected successful outcome with significant improvement in ODI scores and positive patient satisfaction scores. However, the two groups did not differ in self-reported disability scoresor postoperative satisfaction. 
9:36 - 9:41 am

\section{J.A.N.E. AWARD PRESENTATION \\ A Novel Method to Classify Patients with Cervical Incomplete SCI based on Potential for Recovery: A Trajectory Analysis}

Jetan H. Badhiwala MD; Jefferson R. Wilson MD, PhD; Michael G. Fehlings MD, PhD, FRCS(C), FACS

Introduction: The outcomes of cervical incomplete spinal cord injury (SCl) are heterogeneous.

Methods: Patients with cervical incomplete SCI (AIS B-D; C1-C8) were identified from four prospective, multicenter SCI datasets (NACTN; STASCIS; Sygen; NASCIS III). A group-based trajectory model was fit to longitudinal upper extremity motor scores out to 1-year follow-up. Multivariable multinomial logistic regression was performed to identify baseline features that characterize each trajectory group.

Results: In total, 801 patients were eligible. Four distinct trajectory groups were identified:

1. 'Poor outcome': Severe neurological injury with very minimal, gradual recovery.

2. 'Moderate recovery': Moderate-to-severe neurological injury with moderate recovery.

3. 'Good recovery': Moderate neurological injury with good recovery.

4. 'Excellent outcome': Mild neurological injury with good recovery by 3 months.

On adjusted analyses, older age was associated with lower likelihood of an 'excellent outcome' $(P=0.020)$. Compared to AIS B injuries, AIS C injuries were associated with 'moderate recovery' $(P<0.001)$, 'good recovery' $(P<0.001)$, and 'excellent outcome' $(P<0.001)$, and AIS D injuries were significantly associated with 'good recovery' $(P<0.001)$ and 'excellent outcome' $(P<0.001)$. Mid cervical injuries occurred more frequently in 'moderate recovery' $(P<0.001)$, 'good recovery' $(P<0.001)$, and 'excellent outcome' $(P<0.001)$ groups, as compared to upper cervical injuries. The presence/absence of central cord syndrome did not predict temporal recovery profile. Early surgical decompression ( $<24 \mathrm{hrs}$ ) was independently associated with an increased propensity for 'good recovery' $(P=0.039)$ and 'excellent outcome' $(P=0.048)$.

Conclusion: Patients with cervical incomplete $\mathrm{SCI}$ demonstrate distinct trajectories of recovery in upper limb motor function. The trajectory a patient is likely to follow may be predicted by baseline characteristics. The presence of central cord syndrome does not impact prognosis, whereas early surgery may support conversion to a more favorable recovery trajectory. 
9:48 - 9:53 am

\section{MAYFIELD CLINICAL SCIENCE AWARD PRESENTATION Load Sharing Classification Score in Patients with TLICS 4 is Predictive of Surgical Intervention}

Nima Alan; Joseph Donohue; Alp Ozpinar MD; Nitin Agarwal MD; Adam S. Kanter MD; David O. Okonkwo MD PhD; David Hamilton;

Introduction: MRI in patients with TLICS 4 may determine the presence of posterior ligamentous complex (PLC) injury, which could theoretically further advocate for surgery. It is not clear what factors affect the decision making process. Load Sharing Classification (LSC) score, was first used to categorize three column fractures based on the load sharing capacity of affected segment of spine. LSC has never been applied to patients with TLICS 4.

Methods: IRB-approaced observational retrospective cohort study of 111 neurologically intact patients with isolated thoracolumbar burst fracture with TLICS 4 were evaluated at a level I trauma center from 2012-2018. LSC score was determined based on degree of comminution (1-3), apposition (1-3), and kyphosis (1-3), total composite score of 3-9.

Results: The patients were predominantly male (59\%), average age of 61 years, most commonly suffered from motor vehicle accident (34\%). 44 patients underwent MRI and 15 were found to had PLC injury. Overall, 32 patients had surgery. LSC score was higher in patients who had an MRI $(5.6$ vs $3.9, p<0.001)$ and patients who had surgery (6.8 vs $3.7, p<0.001)$ but similar in patients who had PLC injury on MRI $(6.1$ vs $5.4, p=0.1)$. In univriatie ligistic regression LSC score was associated with patients undergoing MRI (OR 1.7, 1.3- 2.1), having PLC injury on MRI (OR 1.5, 1.2- 2.0) and, in multivariate logistic regression, undergoing surgical intervention (OR 3.7, 2.3-5.9), independent of MRI or PLC injury.

Conclusion: LSC in neurologically intact patients with isolated thoracolumbar burst fracture with TLICS 4 is predictive of operative intervention independent of MRI acquisition, and presence of posterior ligamentous injury on MRI. 